\title{
Basal cell carcinoma
}

Citation for published version (APA):

Brinkhuizen, T. (2016). Basal cell carcinoma: epigenetics and new treatment modalities, Out of the box . [Doctoral Thesis, Maastricht University]. https://doi.org/10.26481/dis.20160624tb

Document status and date:

Published: 01/01/2016

DOI:

$10.26481 /$ dis.20160624tb

Document Version:

Publisher's PDF, also known as Version of record

\section{Please check the document version of this publication:}

- A submitted manuscript is the version of the article upon submission and before peer-review. There can be important differences between the submitted version and the official published version of record.

People interested in the research are advised to contact the author for the final version of the publication, or visit the DOI to the publisher's website.

- The final author version and the galley proof are versions of the publication after peer review.

- The final published version features the final layout of the paper including the volume, issue and page numbers.

Link to publication

\footnotetext{
General rights rights.

- You may freely distribute the URL identifying the publication in the public portal. please follow below link for the End User Agreement:

www.umlib.nl/taverne-license

Take down policy

If you believe that this document breaches copyright please contact us at:

repository@maastrichtuniversity.nl

providing details and we will investigate your claim.
}

Copyright and moral rights for the publications made accessible in the public portal are retained by the authors and/or other copyright owners and it is a condition of accessing publications that users recognise and abide by the legal requirements associated with these

- Users may download and print one copy of any publication from the public portal for the purpose of private study or research.

- You may not further distribute the material or use it for any profit-making activity or commercial gain

If the publication is distributed under the terms of Article $25 \mathrm{fa}$ of the Dutch Copyright Act, indicated by the "Taverne" license above, 


\section{Basal Cell Carcinoma}

EPIGENETICS AND NEW TREATMENT MODALITIES

OUT OF THE BOX 
Printing of this thesis was financially supported by:
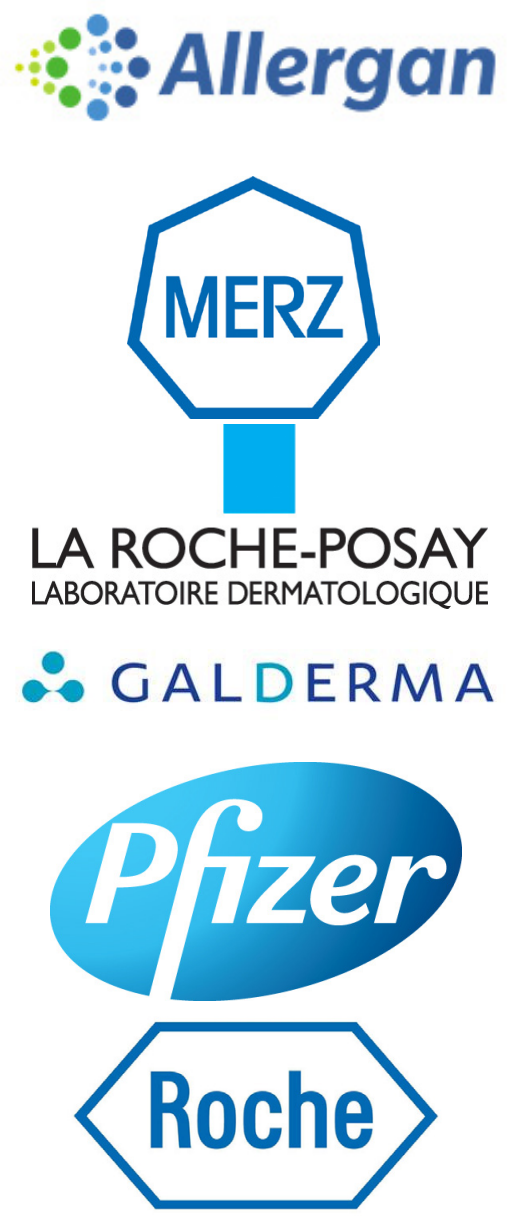

LA ROCHE-POSAY LABORATOIRE DERMATOLOGIQUE
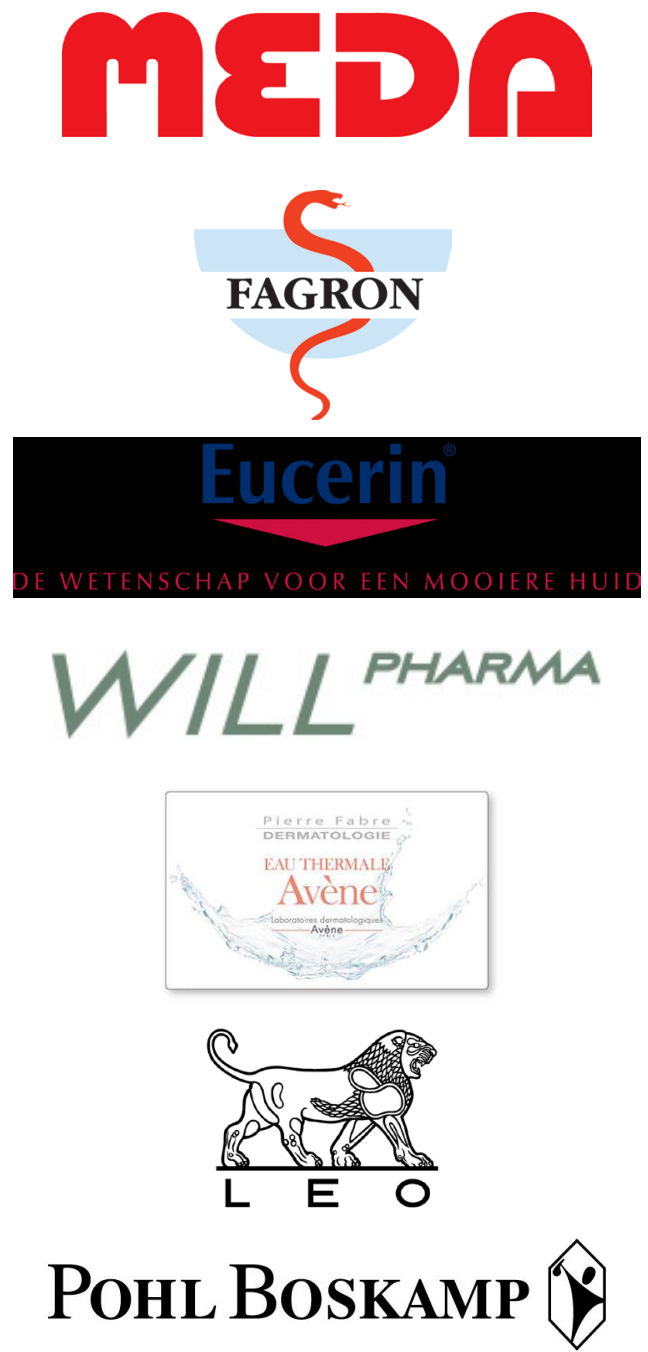

ISBN: 978-94-6233-265-2

Copyright Tjinta Brinkhuizen, Maastricht 2016

Cover design and lay out Evelien Jagtman (evelienjagtman.com)

Printing Gildeprint, Enschede, the Netherlands

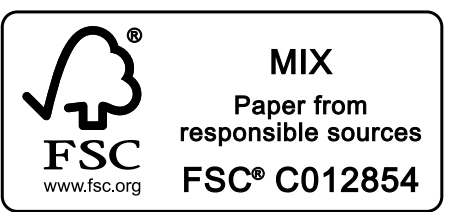




\title{
Basal Cell Carcinoma \\ EPIGENETICS AND NEW TREATMENT MODALITIES \\ OUT OF THE BOX
}

\author{
PROEFSCHRIFT
}

ter verkrijging van de graad van doctor aan de Universiteit Maastricht, op gezag van de Rector Magnificus, Prof. Dr. L.L.G. Soete

volgens het besluit van het College van Decanen,

in het openbaar te verdedigen

op vrijdag 24 juni 2016 om 12.00 uur

door

\section{Tjinta Brinkhuizen}

Geboren 15 november 1982 te Maastricht 


\section{Promotoren}

Prof. Dr. M.A.M. van Steensel

Prof. Dr. P.M. Steijlen

\section{Copromotor}

Mw. Dr. K. Mosterd

\section{Beoordelingscommissie}

Mw. Prof. dr. C.T.R.M. Stumpel (voorzitster)

Mw. Prof. Dr. M.R. van Dijk (Universitair Medisch Centrum Utrecht)

Prof. dr. B. Kremer

Prof. dr. M.A. de Rie (Academisch Medisch Centrum)

Prof. dr. E.J.M. Speel 
Veur mien awwers 



\section{Contents}

Chapter 1

General introduction

PART ONE

PATHOGENESIS OF BASAL CELL CARCINOMA

Chapter 2

2.1

Epigenetic changes in basal cell carcinoma affect $\mathrm{SHH}$ and

WNT signaling components

Functional epigenomics implicates inflammatory signaling in basal cell carcinoma pathogenesis ation and transcriptomic profile

Chapter 3 Immunohistochemical analysis of the mechanistic target of rapamycin and hypoxia signaling pathways in basal cell carcinoma and trichoepithelioma

PART TWO

NEW TOPICAL AND SYSTEMIC TREATMENT MODALITIES FOR BASAL CELL CARCINOMA

Chapter 4

4.1

The effect of topical diclofenac 3\% and calcitriol $3 \mu \mathrm{g} / \mathrm{g}$ on superficial basal cell carcinoma (sBCC) and nodular basal cell carcinoma (nBCC): A phase II, randomized controlled trial manual quantification of immunohistochemical stains in basal cell carcinoma

Chapter 5

5.1

Acquired Resistance to the Hedgehog Pathway Inhibitor 159 Vismodegib due to smoothened Mutations in Treatment of locally advanced basal cell carcinoma ance in a patient treated with vismodegib for locally advanced basal cell carcinoma General discussion \& valorization 
Chapter 8

Grants and awards

List of publications

Oral \& poster presentations

Dankwoord 


\section{Chapter 1}

General introduction 


\section{Skin Cancer}

Skin cancer is the most common malignancy in Caucasians worldwide. ${ }^{1}$ In literature, skin cancer is often divided into malignant melanoma and nonmelanoma skin cancers (NMSC). Of these, melanoma is the most aggressive and deadly and comprises $11 \%$ of all skin cancers. ${ }^{2}$ The term NMSC (or keratinocyte carcinomas) is mainly used for basal cell carcinoma (BCC) and squamous cell carcinoma (SCC). Other primary cutaneous malignancies in the group of NMSC are cutaneous lymphomas, adnexal tumors, Merkel cell tumors and other rare cutaneous neoplasms. ${ }^{1}$ Approximately $80 \%$ of all NMSCs are BCCs, while $10-20 \%$ is SCC. The large number of BCCs to be treated and the correspondingly rising impact on the workload of dermatologists imposes a significant burden on health care systems. Hence, BCC is also a serious health problem. Generally, BCCs are slow-growing indolent tumors with low metastasis rates $(0.0028 \%$ to $0.55 \%)$ and they are rarely fatal. ${ }^{3}$ However, when incorrectly diagnosed and managed or left untreated, BCCs can be locally destructive, causing substantial morbidity and even mortality. In those cases, they have the potential of progressing to a(n) (locally) advanced BCC (I)aBCC). ${ }^{4}$ In this thesis, studies focused on the pathogenesis and treatment of both non-aggressive BCCs and (I)aBCCs.

\section{Epidemiology}

$\mathrm{BCC}$ is the most common cancer among Caucasians with worldwide incidence rates rising by about 3-10\% annually, reaching epidemic proportions. ${ }^{5,6}$ Incidence rates are estimated because NMSCs are generally poorly registered. In many countries, often only the first BCC in a patient is reported and no distinction is made between BCCs and SCCs. ${ }^{7}$ In the Netherlands, a large observational study found a nearly fourfold increase in age-standardized incidence rates from 40 to 165 per 100.000 males and from 34 to 157 per 100.000 females between 1973 and 2009. ${ }^{5}$ One in five to six Dutch citizens will develop at least one BCC during their lifetime. ${ }^{7}$ In the five years following the first $\mathrm{BCC}$, the cumulative risk of developing subsequent $\mathrm{BCC}(\mathrm{s})$ is approximately $40-50 \%$, with the highest risk in the first 6 months after diagnosis of the first BCC. ${ }^{1,8}$ Although BCC is generally considered to be a disease of the elderly, recent literature shows the most rapid increase in its incidence in women aged 40 years or less. ${ }^{5}$ The rising incidence is mainly caused by an increase in recreational (intermittent) ultraviolet radiation ( $U V(R))$ exposure and exposure during childhood. ${ }^{9}$ Other contributing factors are increased patient and physician awareness, better tumor coding and a rise in life expectancy over the years. ${ }^{9}$ BCC mostly affects sun-exposed areas of the skin like the head and neck region. However, the results of a 37-year Dutch observational study show a steep increase in BCCs located on the trunk ${ }^{5}$, possibly caused by sunbathing habits that have gained popularity since Coco Chanel returned suntanned after her holiday in the $1920 \mathrm{~s}^{10}$ 
The importance of UVR for NMSC is further reflected by the geographic variation in incidence, with incidence increasing as latitude decreases. ${ }^{1}$ With the highest amount of UV exposure, Australia has the highest incidence rates, with 935 of 100.000 persons per year being affected, according to a large observational study between 1997 and 2006. ${ }^{11}$ It is estimated that two out of three Australians will develop skin cancer. However, because of poor BCC registration in Australia, true incidence rates are unknown. ${ }^{12}$

\section{Risk factors}

The risk of developing NMSC, including BCC, depends on a number of intrinsic and extrinsic risk factors. The major and best-known risk factor for BCC development is exposure to UVR. Consequently, persons with a light skin, eye and hair color (Fitzpatrick skin types I and II) and those unable to tan have a higher risk of developing skin cancer. There is a typical latent period of years to even decades between UV exposure and the occurrence of BCC. ${ }^{9}$ However, the exact relationship between UV exposure and BCC is rather complex; literature suggest that both exposure early in life and intermittent (i.e. holidays) and cumulative (i.e. occupational related) UV exposure are important. ${ }^{9,13}$ UVR is divided into UVA (320-400 nm), UVB (290-320nm, sunburn rays), and UVC (200-290 nm), based on wavelengths. While UVC is known to be the most harmful, almost $100 \%$ of all solar UVC is absorbed by the atmosphere and the ozone layer. In contrast, even though over 95\% of UVA and only $1-10 \%$ of UVB radiation reaches the earth's surface ${ }^{14}$, UVB is demonstrated to be 10000 times more mutagenic than UVA. This is because UVB directly causes DNA and RNA damage resulting in a characteristic $\mathrm{C} \rightarrow \mathrm{T}$ or $\mathrm{CC} \rightarrow \mathrm{TT}$ transition mutation (UV-signature). About $50 \%$ of all sporadic BCC harbor specific UV-induced mutations in the TP53 tumor suppressor gene. ${ }^{15}$ Still, the underlying mechanisms of UVA-induced DNA damage are less well understood. ${ }^{16}$ Primarily, UVA induces mutations indirectly by generating reactive oxygen species capable of causing oxidative DNA damage. ${ }^{1,16,17}$ However, a rather high proportion of BCCs occur on body sites that are generally less exposed to the sun ${ }^{5,13}$, suggesting that UV radiation alone is not (always) sufficient for the development of BCCs. Photosensitizing medications, such as tetracyclines, thiazide diuretics, NSAIDs and retinoids make the skin more vulnerable to UV damage and in fact increase the risk of especially early-onset BCC. An increased risk is also found after exposure to ionizing radiation in the form of radiotherapy or after exposure to arsenic, which can be present in groundwater. Immune-compromised patients, caused for example by HIV seropositivity or organ transplantation, have a doubled and tenfold risk of BCC development respectively. BCC development is also associated with genetic disorders, such as basal cell nevus syndrome (BCNS), xeroderma pigmentosum (XP) and Bazex-Dupré-Christol syndrome. ${ }^{1,18}$ Of these, the most common syndrome is BCNS, an autosomal dominant disorder causing distinct morphological features including multiple BCCs already at a young age, palmar or plantar pits, jaw cysts tumors and medulloblastomas. ${ }^{19}$ The genetic background of BCNS will be further elaborated below. 


\section{Pathogenesis}

\section{Cells of origin}

BCCs are epithelial tumors histologically resembling the basal cells of the epidermis and the outer layer of the hair follicle. Although BCCs are generally referred to as hair follicle tumors, the real origin of the tumor is still controversial. Reliable cell model systems are not available. Transgenic mice expressing the oncogene M2SMO in skin under control of the keratin 5 (K5) promoter developed lesions resembling superficial BCC (sBCC). These were very similar to embryonic hair germs, comprising a focal group of epidermal cells protruding into the underlying dermis of volar skin completely devoid of follicles or other skin appendages. ${ }^{20} \mathrm{~A}$ few years ago, it was demonstrated in the same mouse model that these tumors derive from long-lived progenitor cells residing in the interfollicular epidermis (IFE) and not from hair follicle bulge stem cells. ${ }^{21}$ More recently, it was shown in a conditional PTCH1 knockout ( via keratin 14 (K14) promoter) mouse that when BCC-like tumors arise from the IFE, this was only from the touch dome epithelia ${ }^{22}$, an IFE niche specialized for mechanosensory signaling. ${ }^{23}$ On the other hand, Peterson et al. also demonstrated that BCClike lesions can originate from multiple hair follicle stem cells (upper bulge, lower bulge and isthmus) and all these tumors displayed a similar phenotype, irrespective of cellular origin. In contrast, most stem cells within the IFE did not efficiently form tumors, not even after loss of TP53.22 The importance of the hair follicle stem cells in the development of BCCs is also confirmed by Kasper et al. showing that after deletion of PTCH1 in hair follicle stem cells in Lgr5Cre-Ptch $1^{\mathrm{fllt} / \mathrm{l}}$ mice, BCCs arise from Lgr5+ stem- and progenitor cells in the hair follicle bulge and secondary hair germ, but not from the IFE. ${ }^{19}$ From the previous, it can be speculated that the histological phenotype of BCC reflects its cellular origin as it was previously described that SBCCs arise from the IFE, whereas nBCCs may originate from the hair follicle. ${ }^{24}$

\section{Genetics and signaling pathways}

Although the molecular mechanisms involved in the pathogenesis of sporadic BCCs remain to be fully elucidated, most, if not all, BCCs are characterized by aberrant activation of the Sonic Hedgehog $(\mathrm{SHH})$ pathway due to mutations in one or more of its component genes. ${ }^{25}$ A schematic overview of the essential signaling pathways in BCCs and a more detailed description of their mode of action is presented in figure 1. The $\mathrm{SHH}$ pathway is essential for embryonic development and in adult tissues it regulates tissue stem cells required for organ repair and maintenance. ${ }^{26}$ Also, $\mathrm{SHH}$ plays an essential role in the progression of adult hair follicle morphogenesis past the hair germ stage of development, though $\mathrm{SHH}$ is not required for its initiation. ${ }^{27}$ Loss of growth control after aberrant activation of $\mathrm{SHH}$ signaling underlines its importance in adult tissues. ${ }^{26}$ The identification of a germline mutation in the tumor suppressor gene Patched-1 (PTCH1) on chromosome 9q22 in BCNS patients revealed the importance of constitutive $\mathrm{SHH}$ signaling in BCC development. ${ }^{19,25}$ In sporadic BCC, inactivating PTCH1 mutations are found in up to $90 \%$ of the patients and an additional $10 \%$ have activating mutations in the oncogene Smoothened (SMO). ${ }^{25}$ Mutations in Suppressor of fused (SUFU) have been reported in only a few cases. ${ }^{28}$ Normally, in the absence of SHH 
ligand, the transmembrane receptor protein PTCH1 inhibits the transmembrane receptor protein SMO and thereby prevents activation of $\mathrm{SHH}$ signaling. Binding of a $\mathrm{SHH}$ ligand or loss of functional PTCH1 releases the inhibition of SMO, causing nuclear translocation of the transcription factors GLI1 and GLI2 through a series of interacting proteins, including SUFU, which results in subsequent transcription of tumor-promoting genes. ${ }^{25,26}$ Interestingly, the $\mathrm{SHH}$ pathway is also activated by sensory nerves in normal touch domes, while nerve ablation and consequent loss of $\mathrm{SHH}$ activity attenuates touch dome-derived tumors. This could implicate that cutaneous nerves are important regulators of stem cell niches. ${ }^{22}$

Another important pathway associated with hair follicle development and cycling as well as with (skin) tumor development is canonical (ß-catenin dependent) Wingless (WNT) signaling. ${ }^{29}$ Canonical WNT signaling initiates hair bud formation, while subsequent $\mathrm{SHH}$ signaling is required for maturation of the hair follicle by promoting the proliferative expansion of the follicle epithelium. ${ }^{20}$ Murine BCC models showed that WNT signaling is essential for the tumorigenic response to deregulated $\mathrm{SHH}$ signaling in the skin (figure 1). ${ }^{20,29}$ This activation of canonical WNT signaling in BCC is suggested by cytoplasmic and nuclear localization of $\beta$-catenin in BCC immunohistochemical stains ${ }^{30}$, though the accuracy of $\beta$-catenin immunohistochemical stains as indicator of active WNT signaling is debatable. Non-steroidal anti-inflammatory drugs (NSAIDs) can suppress the WNT pathway by reducing nuclear $\beta$-catenin localization and, consequently, attenuating transcription of target genes in colorectal cell lines. ${ }^{31,32}$ However, the main mode of action of NSAIDs seems to be the suppression of elevated cyclooxygenase (COX) enzyme activity. There are two COX isoforms, COX-1 and COX-2, and it is COX-2 that is elevated in several solid tumors, but also in epithelial cancers like BCC. ${ }^{32,33}$ Additionally, UVR increases COX-2 expression in the epidermis. ${ }^{34} \mathrm{COX}-2$ catalyzes the synthesis of prostaglandins from arachidonic acid. Prostaglandin E2 (PGE2) is increased by UV-induced COX-2 expression. PGE2 is one of the major cyclooxygenase products implicated in UV-induced skin carcinogenesis by increasing tumor cell proliferation, inhibition of apoptosis, stimulation of an inflammatory response, promotion of immunosuppression and facilitation of tumor invasion. ${ }^{34}$ Inhibition of COX-2 by NSAIDs leads to apoptosis, mostly by blocking the anti-apoptotic Bcl-2 protein and by activation of the caspase cascade. ${ }^{35,33}$ Interestingly, in a double-blinded, randomized 3-year clinical trial studying the effect of oral Celecoxib (200 mg, twice daily, 24 months) on the development of BCC in 60 patients with BCNS, a trend was found towards reduction in BCC burden in the treatment group ( $p=0.069$ ). When considering only the $60 \%$ of patients with less severe disease, Celecoxib actually did significantly reduce BCC number and burden $(p=0.024) .{ }^{36}$ Topical 3\% diclofenac in $2.5 \%$ hyaluronic acid is already approved by the U.S. Food and Drug Administration (FDA) in many European countries, though not in the Netherlands, as treatment of actinic keratosis (AK): pre-cancerous lesions of which it is still assumed that they have the potential to develop into SCC. ${ }^{37} \mathrm{~A}$ double-blind, randomized, placebo-controlled multicenter study on the efficacy of twice daily application of $3 \%$ diclofenac in $2.5 \%$ hyaluronic acid for 60 days in patients with AK showed response rates of $79 \%$ versus $45 \%$ and complete clearance in $50 \%$ versus $20 \%$ in the treatment group versus the control group $(p<0,001 \%)$ respectively. ${ }^{38}$ It is becoming more evident that drugs already available for diseases other than cancer could 
serveas anti-cancer agents. Of special interestis the comprehensivemode of action of vitaminD.

Vitamin D signaling and its potential role in the pathogenesis of various human cancers is of increasing interest in cancer research (figure 1). 1a,25(OH)2D3 (calcitriol) is the active metabolite of vitamin $\mathrm{D}$, with antiproliferative and pro-apoptotic effects. ${ }^{39}$ Calcitriol can also inhibit angiogenesis and it has shown synergistic effects in cancer treatment in combination with cytotoxic anticancer agents and NSAIDs. ${ }^{39}$ In addition to these direct effects of calcitriol, the vitamin $\mathrm{D}$ receptor (VDR) is thought to be crucial in reducing the proliferative action of tumors by indirectly functioning as a tumor suppressor. Activation of the VDR by calcitriol induces the expression of the transmembrane protein E-Cadherin, which recruits $\beta$-catenin to the cell membrane, thereby preventing translocation of $\beta$-catenin to the nucleus and so inhibiting canonical WNT signaling. Also, calcitriol directly inhibits SMO in vitro resulting in Gli-mediated repression of $\mathrm{SHH}$ signaling. ${ }^{39,40}$ 


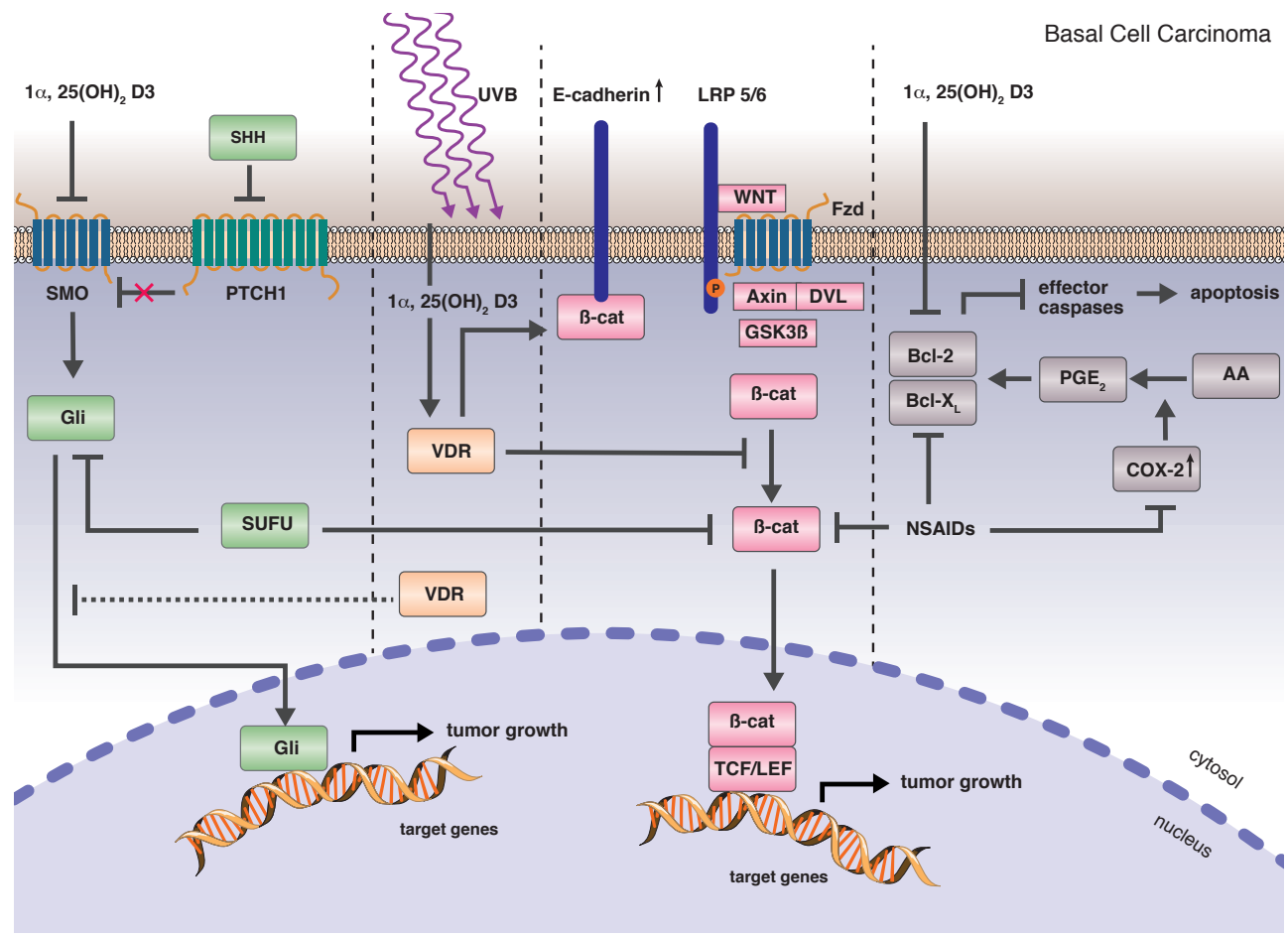

Brinkhuizen T, Frencken KJA

Figure 1. Actions of NSAIDs and Calcitriol in Basal Cell Carcinoma: a schematic overview.

Sonic Hedgehog Pathway (green). The extracellular protein Sonic hedgehog (SHH) binds to and inhibits Patched (PTCH1), a transmembrane receptor, which relieves the inhibition of another transmembrane protein, Smoothened (SMO). SMO activates glioma-associated oncogene homolog 1 (GLI1) and GLI2, transcription factors that travel into the nucleus to activate the expression of tumor-promoting genes. ${ }^{25}$ Canonical WNT signaling pathway (pink). Binding of a WNT ligand to its specific receptor complex containing a Frizzled (FZD) family member and LRP5 or LRP6 co-receptors, initiates WNT- $\beta$-catenin signaling. Axin relocates to the LRP $5 / 6$ tail at the membrane that is bound to WNT through its interaction with dishevelled (DVL), which forms a complex with GSK3 $\beta$ and prevents $\beta$-catenin ( $\beta$-cat) degradation. ${ }^{31}$ This allows $\beta$-catenin to accumulate and enter the nucleus, where it interacts with members of the TCF/LEF family. In the nucleus, $\beta$-catenin converts the TCF proteins into transcriptional activators. Suppressor of fused (SUFU) functions as a tumor suppressor by inhibiting both SHH and WNT signaling..$^{20}$ NSAIDs inhibit WNT signaling by reducing nuclear $\beta$-catenin localization. ${ }^{31,32}$ Furthermore, NSAIDs inhibit cyclooxygenase-2 (COX-2), which is overexpressed in basal cell carcinoma (BCC) and catalyzes the conversion of arachidonic acid (AA) to prostaglandins (PGE2). A subsequent reduction of PGE2 and a direct down regulation of the anti-apoptotic $\mathrm{Bcl}-2$ family proteins by NSAIDs induces apoptosis. ${ }^{33} \mathrm{~A}$ down regulation of $\mathrm{Bcl}-2$ is also induced by $1 \mathrm{a}, 25(\mathrm{OH}) 2 \mathrm{D} 3$ (calcitriol), resulting in caspase cleavage leading to apoptosis. Calcitriol directly inhibits SMO in vitro, resulting in repression of $\mathrm{SHH}$ signaling. SHH-signaling is also suggested to be directly repressed by the Vitamin D Receptor (VDR) by inhibition of GLI. Finally, activation of the VDR by calcitriol induces the expression of the transmembrane protein E-Cadherin, which recruits $\beta$-catenin to the cell membrane and prevents translocation of $\beta$-catenin to the nucleus. ${ }^{39-41}$ 


\section{Epigenetics}

Epigenetics refers to hereditary alterations by mechanisms other than changes in the nucleotide sequence of a gene. It has been suggested that epigenetic modifications occur at least as frequently as genetic mutations in the development of cancer. ${ }^{42}$ Epigenetic modifications are considered to be more tumor specific, while genetic alterations are generally patient specific. ${ }^{43}$ Of these, DNA-methylation is the best-known, consisting of the addition of a methyl group to cytosine nucleotides followed by a guanine (C-phosphate-G (CpG) dinucleotide), catalyzed by enzymes called DNA methyltransferases (DNMTs). ${ }^{44} \mathrm{CpG}$ dinucleotides can be found in clusters ( $\mathrm{CpG}$ islands), mostly in the promoter region of the gene, which is where DNA transcription is initiated. CpG islands are generally hypomethylated, while the CpG dinucleotides in the majority of the genome are hypermethylated to prevent chromosome instability. $\mathrm{CpG}$ island hypermethylation is associated with transcriptional silencing, which in the case of tumor suppressor genes could contribute to the development of cancer. ${ }^{44}$ In cancer cells a shift in the distribution of DNA-methylation can be detected: hypermethylation is mainly found in the promoter regions, while the rest of the genome exhibits general hypomethylation. Besides its role in carcinogenesis, DNA-methylation is also instrumental in the inactivation of the X-chromosome and genomic imprinting. ${ }^{45}$ Epigenetic alterations in $\mathrm{BCC}$ have previously been reported, but only in limited sample series and without providing further experimental evidence for the relevance of these changes for BCC carcinogenesis. ${ }^{46-50}$

\section{Immunology}

There is increasing evidence that interactions between tumor cells and the surrounding stroma play an important role in BCC development and progression. ${ }^{51}$ The peritumoral stroma of BCCs is well-defined and is composed largely of activated fibroblasts, inflammatory cells and vasculature. Soluble paracrine factors such as WNT-1, HGF and TGF- 3 are secreted by stromal fibroblasts and generally mediate the complex interplay of the stroma with the developing tumor. ${ }^{52}$ In addition to these paracrine factors, inflammatory cells in the peritumoral stroma can secrete growth factors and reactive oxygen species, which further contribute to tumor growth. ${ }^{52,53}$ Several studies support the idea that BCCs are stroma-dependent and that their growth is limited by the host immune response. They fail to grow in culture and transplantation of human BCC biopsies into mice is only successful in T, B and natural killer (NK) cell deficient mice. ${ }^{54}$ Quantification of the relationship between BCC subtypes and their peritumoral stroma revealed an association between the infiltrative subtype and thicker stroma. Also, elastosis was found more frequently in infiltrative BCCs, which could possibly be explained by the presence of extensive sun damage. So peritumoral stroma seems to differ between the different BCC subtypes. ${ }^{52}$

Over the past three decades, the importance of inflammation in driving tumor progression has been underscored by the chemopreventive effect of NSAIDs targeting COX in several human tissues. Presence of COX allows maturating dendritic cells (DC) to produce interleukin (IL) 12 and so mediate a Th1 adaptive immune response, while in the absence of COX a Th2 response is initiated. ${ }^{55}$ In brief, the Th1 response is pro-inflammatory whereas the Th2 is 
essentially anti-inflammatory and promotes allergic responses. ${ }^{56}$ DCs are important (tumor) antigen presenting cells in the skin and they are thought to be crucial in generating an aggressive immune response against cutaneous cancers. ${ }^{51}$ This makes DCs attractive targets for immunotherapies, though most trials investigating the effect of DC vaccines in various human cancers have shown minimal clinical success with respect to tumor regression and overall survival rates. ${ }^{57}$ In BCCs, elevated amounts of DCs were found in the dermis and in the tumor after treatment with imiquimod (an imidazoquinoline), resulting in more efficient tumor clearance. ${ }^{57}$ Comparable enhanced cell proliferation is seen during wound healing after tissue injury. ${ }^{53}$ Interestingly, biopsy-induced regression of BCCs is a well-known clinical observation which, in one study, was seen in up to $20 \%$ of cases, with a mean interval of 46 days. ${ }^{58}$ The Th1 initiated immune response is suggested to be essential in the promotion of spontaneous regression after a biopsy ${ }^{59}$, which is in line with the induced Th1 response after topical application of imiquimod. ${ }^{60}$ Conversely to the initiated Th1 response, Kaporis et.al. propose the BCC tumor microenvironment to be predominantly Th2 dominant which suggests a dynamic state within the immune microenvironment associated with BCC. ${ }^{60}$ It is known that SCCs but also BCCs are prone to occur in areas of the skin with chronic ulceration, irritation or inflammation; the so called Marjolin's ulcers. ${ }^{61}$ While the exact pathogenesis of this malignant degeneration remains unknown, an attenuated immune response is expected due to chronic inflammation (causing hyperproliferation and DNA-damage), recurrent ulceration and healing, damage of lymph-and blood vessels, indurations and scarring. ${ }^{62}$

Apparently, inflammatory cells have a dual role in BCCs development by, on the one hand, mechanisms like releasing growth/survival factors, promoting (lymph-)angiogenesis and stimulating DNA damage, and, on the other hand the antitumor effects of anti-inflammatory cytokines. So, the challenge for the future is to selectively suppress pro-inflammatory cytokines and increase those cytokines that are anti-inflammatory. ${ }^{53}$

\section{Clinical presentation and diagnosis}

In general, BCCs are easy accessible for clinical evaluation and a trained dermatologist can often diagnose BCCs based on their macroscopic appearance. However, differentiation between the various subtypes can be challenging. Several classification systems have been devised, generally lacking uniformity of terminology and a clear definition. ${ }^{63} \mathrm{~A}$ commonly used simplified classification divides BCC into four subtypes: superficial BCC ( $\mathrm{BBCC}$ ); (micro) nodular ('solid') BCC ( $\mathrm{BCCC}$ ); infiltrative BCC (iBCC), including morphoeic type, mixed type and a combination of the aforementioned types. ${ }^{63}$ An even more simplified system divides BCCs into indolent-growth (including $\mathrm{SBCC}$ and $\mathrm{nBCC}$ ) or aggressive growth (including iBCC, metatypical and morphoeic) subsets. ${ }^{64}$ Differentiation between the BCC subtypes is important since the different histological subtypes are associated with altered biologic behavior and risk of recurrence after inadequate treatment. Although the diagnosis of a BCC is primarily based on clinical features, a punch biopsy is recommended to confirm the diagnosis and histological subtype. ${ }^{64}$ More than one subtype is seen in 
$18-49 \%$ of the $\mathrm{BCCs}^{64-66}$ and it is the most aggressive one that determines the appropriate treatment. ${ }^{67}$ An adequately taken biopsy correctly predicts the most aggressive subtype of the entire BCC in $84.4 \%$ of the cases and tumors with missed aggressive areas on a biopsy have a higher risk of recurrence. ${ }^{68}$ The overall agreement between BCC histological subtype on punch biopsy and on the subsequent surgical excision of primary BCC is only $60.9 \% .{ }^{68}$ The three most relevant subtypes and a more rare clinical presentation of BCC, so-called (I)aBCC, will be discussed in more detail below. Figure 2 presents clinical pictures of the most common BCC subtypes with their histological presentation. 


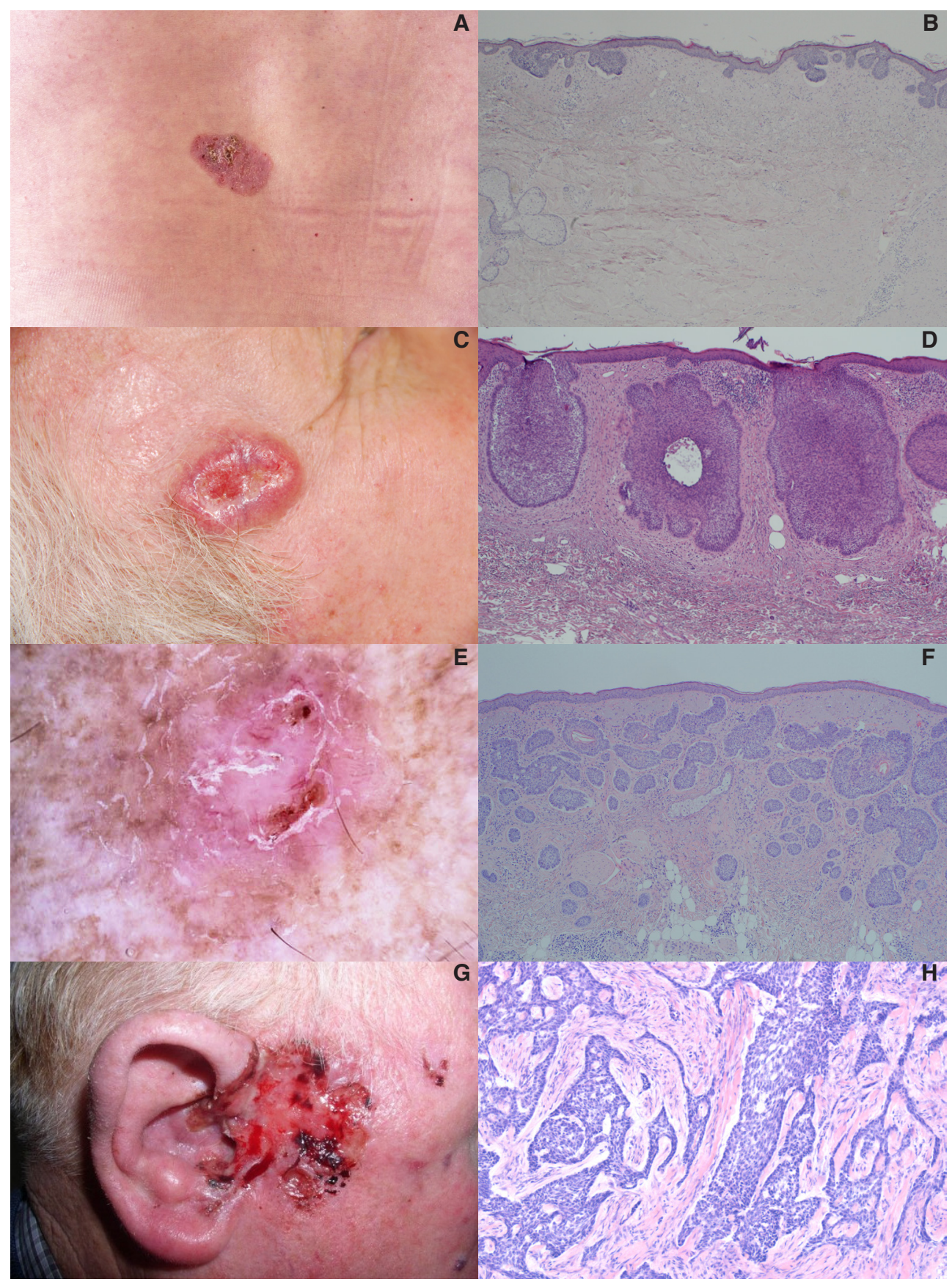

Figure 2. Clinical and histological presentation of basal cell carcinoma (BCC).

A,B. superficial BCC; A clinical and B histological presentation. C,D. nodular BCC; C clinical and D histological presentation. E,F. infiltrative BCC; E clinical and $\mathbf{F}$ histological presentation. G, H. locally advanced BCC; $\mathbf{G}$ clinical and $\mathbf{H}$ histological presentation. 


\section{Indolent-growth subtypes basal cell carcinoma}

$\mathrm{nBCC}$ is the most common subtype and typically presents as a pearly erythematous or skin-colored papule with a raised border and arborizing teleangiectasia. Scaling, crusting, ulceration and bleeding are frequently seen. nBCCs predominantly present in the head and neck region of elderly people. On haematoxylin \& eosin (H\&E) stained histopathology, large nests of basaloid cells with peripheral palisading and a more chaotic arrangement of the central cells are seen in the papillary or reticular dermis. Retraction spaces sometimes form between the tumor islands and the surrounding stroma. The solid nests, as they are often referred to, are stained intense purple (haematoxylin) as the cells have only little cytoplasm. ${ }^{18,69}$ In pigmented BCCs, functional melanocytes are scattered through the tumor islands and a few melanosomes can be found within the tumor cells. In the stroma of pigmented BCCs, numerous melanophages are present. ${ }^{69}$ Characteristic dermoscopy features are large grayblue ovoid nests, multiple gray-blue globules, maple-leaf like areas, and spoke-wheel areas. ${ }^{70}$

sBCC is the second most common subtype, occurring more often at a younger age and frequently located on the trunk. ${ }^{18,71}$ A typical sBCC presents as a slowly growing, well-demarcated, erythematous macule or patch, sometimes with a small erosion and a border of translucent small papules, varying in size from a few millimeters to centimeters. In sBCCs, as in nBCCs, variable amounts of melanin pigment may be present. sBCCs can mimic eczema or psoriasis, while lichenoidkeratosis, actinickeratosisand Bowen's diseasearefurthermaindifferential diagnoses. ${ }^{18}$

Histologically, in sBCCs small buds (mostly multifocal) of proliferating basal cells spread from the undersurface of the epidermis into the papillary dermis with preservation of attachment to the epidermis. There is palisading of the cells at the periphery and slit-like retraction from the subjacent stroma is frequently seen. ${ }^{69}$

\section{Aggressive-growth subtypes basal cell carcinoma}

Infiltrative and morphoeic BCCs present as pink-to-ivory white, shiny, smooth, indurated or depressed atrophic or scar like plaques with poorly defined borders. Although teleangiectasias, erosions, or small crusts may be seen, these signs are frequently absent. Lesions can be missed because their appearance is subtle. Also, they may be misclassified as scar tissue, morphea (localized scleroderma), dermatofibrosarcoma protuberans, Merkel cell carcinoma, amelanotic melanoma, Bowen's disease, eczema, microcystic adnexal carcinoma, and other adnexal neoplasms. ${ }^{18,64}$ Because of their infiltrating behavior, infiltrating and morphoeic BCCs tend to be more aggressive and their propensity to subclinical spread may cause a diagnostic delay. ${ }^{18}$ Histologically, elongated strands of basaloid cells one to eight cells thick extend between collagen bundles in the dermis and even into the subcutaneous fat. Additionally, infiltrative BCCs can also form large nodules. Peripheral palisading is mostly absent and retraction artifacts are uncommon. ${ }^{18,69} \mathrm{~A}$ summary of the prognostic factors classifying BCCs into low-risk versus high-risk categories is presented in table 1. 
Table 1. Prognostic factors determining treatment success in basal cell carcinoma ${ }^{67}$

\begin{tabular}{lll} 
& Low-risk & High-risk \\
Histological growth pattern & $\begin{array}{l}\text { Not-aggressive } \\
\text { (superficial or nodular) }\end{array}$ & $\begin{array}{l}\text { Aggressive } \\
\text { (infiltrative or micronodular) }\end{array}$ \\
\hline Localization & Trunk & $\begin{array}{l}\text { H-zone } \\
\text { (eyes, ears, lips, nasolabial fold, }\end{array}$ \\
Size & $<2 \mathrm{~cm}$ & nose) \\
\hline Previous treatment & Primary tumor & $>2 \mathrm{~cm}$
\end{tabular}

(Locally) advanced basal cell carcinoma

Aggressive-growth BCCs and indolent-growth BCCs that are neglected for many years or inadequately treated have a greater potential of progressing to (I)aBCC. (I)aBCCs are defined as 'basal cell carcinoma of American Joint Committee on Cancer (AJCC) stage II or above ${ }^{72}$, in which current treatment modalities (surgery and radiotherapy) are considered potentially contraindicated by clinical or patient-driven factors'. Stage II BCCs are categorized as T2 tumors (larger than $2 \mathrm{~cm}$ across, or any size with 2 or more high-risk features) according to the AJCC TNM staging system..$^{72}$ Disease factors leading to a diagnosis of advanced BCCs are, amongst others, tumor size, location, subtype and number. Patient age, performance status and quality-of-life-effects of treatment are examples of patient-driven factors (table 2). ${ }^{73}$ (I)aBCCs generally show deep tissue invasion, may metastasize and cause substantial morbidity or even mortality. ${ }^{74}$ Histologically they are indistinguishable from common BCC. Additional diagnostics, such as magnetic resonance imaging (MRI) or computed tomography (CT) are often necessary to evaluate the expansion of the tumor and a multidisciplinary approach is recommended to manage these patients. Frequently, these tumors are not eligible for surgery because free resection margins are not likely to be achieved, or because surgery has a high morbidity or can be expected to be mutilating. Contraindications, such as prior radiotherapy can also be present. Prior radiotherapy, location, size or patients restrictions such as the many visits to the hospital may limit the usefulness of radiotherapy. Also, radiotherapy in BCNS patients with (I)aBCC is contra-indicated, because of the risk of inducing new BCC in the irradiated area. ${ }^{75}$ Treatment with cytotoxic agents such as cisplatin has been reported, but there is insufficient evidence to support their efficacy in the treatment of (I)aBCCs and metastatic BCCs (mBCCs). ${ }^{76}$ As mentioned before, BCCs rarely metastasize, however when metastasis does occur, the prognosis is very poor with mean survival ranging from 8 months to 3.6 years. ${ }^{77}$ Lymphogenic metastasis in regional lymph nodes is most common in these cases, followed by haematogenic metastasis to the lungs and the liver. ${ }^{78}$ The development of SMO inhibitors as treatment for (I)aBCCs and mBCCs is expected to be promising, given that the majority of the BCCs are characterized by aberrantly activated $\mathrm{SHH}$ signaling. SMO inhibition will be discussed in more detail in the following section. 
Table 2. Definition of advanced basal cell carcinoma (BCC), with tumor and patient factors that guide a diagnosis of advanced $\mathrm{BCC}^{73}$

Definition of advanced BCC

'Basal cell carcinoma of American Joint Committee on Cancer (AJCC) stage II or above, in which current treatment modalities are considered potentially contraindicated by clinical or patient-driven factors.'

Factors guiding a diagnosis of advanced BCC

Disease factors

Tumor size (e.g., giant BCC)

Tumor location (e.g., $\mathrm{H}$-zone tumors)

Number of tumors

Tumor subtype (e.g., morphoeic tumors)

Likelihood of successful treatment (e.g., recurrent BCC)

Patient factors

Patient age (e.g., radiotherapy in young patients)

Patient performance status (e.g., frail patients)

Quality-of-life-effects of treatment (e.g., poor cosmetic outcome)

Patient opinions regarding treatment

Presence of genodermatoses with increased risk for cancer (e.g., Gorlin's syndrome)

Presence of comorbidities (e.g., organ transplant)

\section{Treatment}

\section{Common therapies}

For the different types of BCCs, a variety of invasive and non-invasive treatments are available. ${ }^{18}$ Conventional surgical excision is the gold standard in the treatment of all BCCs. ${ }^{67}$ According to the Dutch guideline, surgical excision with a clinical safety margin of $3 \mathrm{~mm}$ in low risk BCCs and $5 \mathrm{~mm}$ in high risk BCCs (table 1) followed by histological examination is an effective treatment. ${ }^{67,79}$ Even though only a small part of the specimen margin is examined, i.e. less than $2 \%^{80}$, recurrence rates, five years after conventional surgical excision, are rather low: $4-10 \%$ for primary BCCs (pBCCs) and up to $12.1 \%-17 \%$ for recurring BCCs (rBCCs) ${ }^{79,81-84}$ The cumulative probability of recurrence after ten years is still high with $12.2 \%$ for pBCCs and $13.5 \%$ for rBCCs. ${ }^{85}$ In contrast to conventional excision, Mohs' micrographic surgery provides a three-dimensional histological visualization and $100 \%$ evaluation of resection margins. ${ }^{86}$ By assessment of horizontal frozen tissue sections during the surgical procedure, complete assessment of the resection margins can be obtained. Hereby, assessment of resection margins is thought to be more accurate and a further advantage is, that because of the use of frozen sections, reconstruction of the defect can be completed on the same day. ${ }^{87}$ Mohs' micrographic surgery is especially suitable for primary high risk BCCs in the face (five-year recurrence rate of 3-6\%) and for rBCCs in the face it is the first-line treatment (five-year recurrence rate of 4,8-10\%). ${ }^{79,84,87}$ After ten years, the cumulative probability of recurrence is $4.4 \%$ for $\mathrm{pBCC}$ and $3.9 \%$ of rBCCs. ${ }^{85}$ The func- 
tional and cosmetic outcomes are generally better after Mohs' surgery in delicate areas of the face, as more healthy skin is preserved than after conventional surgical excision. ${ }^{88}$ Other invasive treatments, such as cryosurgery or curettage followed by electrocoagulation, can be considered for small low risk BCCs. However, in general these treatments are not recommended because of the higher recurrence rates and worse cosmetic outcome. ${ }^{67}$

Radiotherapy is an effective non-invasive treatment for primary BCCs on all body parts, however it is mainly reserved for irresectable tumors or for patients who cannot undergo surgery. ${ }^{89}$ There is only limited evidence on the long term effects, but in two older studies recurrence rates in primary BCCs after two to four years are found to be 4-7.5\%. ${ }^{79,90}$ In rBCCs

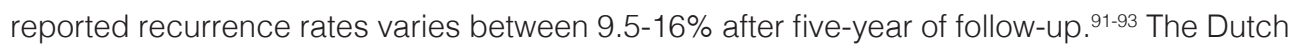
guideline recommends radiotherapy particularly for BCC located in the face or around the ear to preserve cosmesis when surgery isn't desired. ${ }^{67}$ Better cosmetic outcome is achieved with lower dosage fractions during a longer period (17- 20 fractions of 3- 3.5 Gy in 4-5 weeks), associated with less post-radiation atrophy, telangiectasias, fibrosis and necrosis. ${ }^{67}$ As already described above, radiotherapy is contra-indicated in patients with BCNS. ${ }^{75}$

Additional non-invasive treatment modalities which include photodynamic therapy (PDT) (photochemical), imiquimod (immune-modulating) cream and 5-fluoroucil (5-FU) (chemotherapeutic) cream, are preferably only used for sBCC only. Tumor-free survival rates in sBCCs vary from 72.8-84.0\% (PDT), 83.4-87.3\% (imiquimod) and 80.1\% (5-flourouracil), however, they do not reach those of surgical excision. ${ }^{94,95} \mathrm{~A}$ recently published randomized controlled trial investigated the effect of imiquimod cream in nBCCs and found clearance rates of $81.8 \%$ after 3 years. ${ }^{96}$ Non-invasive treatments may have several advantages, such as lower healthcare costs and a decrease in the workload for dermatologists. Also, a better cosmetic outcome with an increase in quality of life for patients can be expected. ${ }^{96}$

\section{Targeted therapies}

Targeted therapies are drugs that can block crucial biochemical pathways or mutant proteins that are required for tumor cell growth and survival. With these, further tumor progression can be prevented and even tumor regression can be induced. ${ }^{97}$ The feasibility of blocking SHH was already suggested in the 1950s by an epidemic of sheep with congenital deformities in Idaho. ${ }^{98}$ Sheep that grazed on wild corn lily (Veratrum californicum) during gestation gave birth to lambs with cephalic defects and cyclopia (one eye). Cyclopamine (11-deoxojervine), a steroidal alkaloid, was identified as the teratogene. ${ }^{99}$ Because of the similarities between cyclopamine induced developmental malformations and holoprosencephaly (one-lobed brain)-like abnormalities associated with loss of $\mathrm{SHH}$ function, it was suggested that cyclopamine is capable of inhibiting the celular response of the $\mathrm{SHH}$ signal. ${ }^{100}$ Taipale et.al. showed in vitro in primary murine fibroblasts lacking functional PTCH $\left(\mathrm{PTCH}^{--}\right)$that cyclopamine inhibits the $\mathrm{SHH}$ pathway by antagonizing SMO. ${ }^{101}$ Furthermore, several mouse models confirmed the effect of cyclopamine on tumor shrinkage. ${ }^{100}$ However, poor oral bioavailability, low affinity for the receptor and inadequate pharmacokinetics ${ }^{102}$ all 
conspire to make cyclopamine less suitable as treatment for BCCs in daily practice. Though, the discovery of the usefulness of cyclopamine as antagonist of SMO has led to the emergence of several synthetic, cyclopamine-like SMO antagonists, the so-called small molecule inhibitors. SHH and GLi molecularly targeted therapies are also upcoming ${ }^{103,104}$ and all these drugs that interfere with the $\mathrm{SHH}$ pathway are frequently referred to as 'Hedgehog Pathway Inhibitors' (HPIs). In 2012 the FDA approved vismodegib 150 mg capsules as first in class for the treatment of (I)aBCCs and mBCCs. ${ }^{105}$ Vismodegib is an orally active synthetic cyclopamine-like SMO-inhibitor. In a phase 2 study, complete and partial response to vismodegib was seen in $43 \%$ and $30 \%$ of (I)aBCCs and mBCCs patients, respectively ${ }^{106}$ and recent follow-up results showed increasing response rates of respectively $47.6 \%$ and 33.3\%. ${ }^{107}$ Tumor responses are generally observed within the first 2 months of treatment but can take up to 10 months to manifest. ${ }^{77}$ Only recently, pre-planned interim results of a large international phase 2 study into the safety of vismodegib became available, showing that at least one adverse event was reported by essentially all patients treated (99\%). However, the reported adverse events were mainly mild or moderate. The most common adverse events included muscle spasms (64\%), alopecia (62\%), dysgeusia (54\%) and weight loss (33\%). Discontinuation of treatment was observed frequently (45\%), however the majority of these patients with mild to moderate adverse events had already achieved partial or complete response when they stopped. Serious adverse events were noted in $22 \% .{ }^{108}$ As the same adverse events are reported in patients treated with sonidegib, another $\mathrm{SHH}$ signaling pathway inhibitor, it seems logical to conclude that they are the result of the direct inhibitory effect on this pathway, a phenomenon that is also known as the class effect. ${ }^{109}$

Despite the reasonable response rates, initial response to vismodegib followed by secondary tumor progression has been observed more and more in BCCs. ${ }^{1,110-113}$ Acquired resistance to vismodegib has also been described in medulloblastoma, caused by a heterozygous SMO mutation found in the metastatic medulloblastoma that developed during treatment. ${ }^{114}$ Atwood et.al. recently showed that in BCCs, a large number of SMO variants are responsible for the maintenance of $\mathrm{SHH}$ signaling in the presence of SMO inhibitors. ${ }^{115}$ More knowledge about the mutational status of pre-treatment and recurrent tumor tissue will help to anticipate on the type of resistance in order to proactively alter treatment. Perhaps in the nearfuture, treatment schedules and combination therapies can then be customized to both patient and tumor characteristics. 


\section{Aims of this thesis}

The content of this thesis is subdivided into two parts:

Part one, which focuses on the pathogenesis of BCC, and part two, in which new topical and systemic treatments of BCCs are described. The main aim of part one is to evaluate epigenetic alterations, i.e. aberrant DNA-methylation, in both non-aggressive and advanced BCCs. Epigenetic alterations in BCCs have previously been reported, but only in limited sample series. ${ }^{46-50}$ Promoter hypermethylation results in gene silencing and can precede genetic mutations and genomic instability in tumor development, and may thus not only be crucial for carcinogenesis, but also represent a potential therapeutic target. ${ }^{44,116}$ Assessing the methylation status of tumor suppressor genes that are expected to be involved in BCCs pathogenesis would provide a first insight into the importance of DNA-methylation in BCC. Additional genome-wide analysis of the BCC epigenome and also RNA- expression levels (transciptome) could further reveal new perspectives in BCC biology, and even in BCC treatment.

Part two aims to address the effectiveness of new topical and systemic treatments in BCCs management, their risks and their use in daily practice. Conventional surgical excision is the gold standard and most effective treatment for all types of BCC. ${ }^{66,117}$ However, lower healthcare costs, a decrease in the workload for dermatologists and a better cosmetic outcome with an increase in quality of life for patients can be expected with non-invasive treatments for low-risk BCCs. ${ }^{117}$ Current research on BCCs focuses on compounds that specifically target key signaling pathways required for tumor growth, however the available non-invasive treatments for low-risk BCCs as such are not specifically targeted. There is increasing evidence that drugs already available for diseases other than cancer, like NSAIDs (anti-inflammation) and calcitriol (psoriasis) can be re-purposed as anticancer-agents. ${ }^{31}$ And since NSAIDs can target the WNT signaling pathway, calcitriol the WNT and SHH pathway and since both drugs can induce apoptosis ${ }^{31,39}$, we assumed that they could have promising therapeutic effects in BCCs. We additionally hypothesized that simultaneously targeting different signaling pathways or pathway elements may have a synergistic effect, as suggested by several (pre-)clinical studies. ${ }^{118} \mathrm{~A}$ combination of NSAIDs and calcitriol would then be a logical step in BCCs treatment.

For (I)aBCCs, an orally active synthetic cyclopamine-like SMO-inhibitor (vismodegib) shows reasonable response rates, however initial response to vismodegib followed by secondary tumor progression has already been observed. ${ }^{110-113}$ Only recently it was shown that various SMO mutations are responsible for the maintenance of $\mathrm{SHH}$ signaling in the presence of $\mathrm{SMO}$ inhibitors. ${ }^{115} \mathrm{~A}$ better insight into the possible rapid occurrence of resistance to vismodegib, apparently caused by different acquired mutations could help to dynamically adapt treatment to both patient and tumor characteristics. 


\section{Part one: pathogenesis of BCC}

Chapter 2 of this thesis provides a comprehensive analysis of genetic, epigenetic and RNA-expression changes in BCCs, and consists of 3 paragraphs. The genetic background of BCC has been studied extensively, while so far its epigenetic makeup has received comparatively little attention. Epigenetic modifications are heritable changes in DNA structure other than alterations in DNA sequence capable of driving malignant tumor development and progression. To date, DNA hypermethylation is the best-characterized epigenetic mechanism, comprising the addition of a methyl group to a cytosine base adjacent to a guanine base (the CG dinucleotide). ${ }^{44}$ CG-rich areas (CpG islands) located in the promoter region of many tumor suppressor genes become hypermethylated in numerous malignancies ${ }^{119}$, which results in gene silencing. ${ }^{120}$ The main hypothesis of this chapter is that DNA-methylation plays a significant role in BCC tumorigenesis and leads to differential expression of the affected genes. In chapter 2.1 we sought to analyze the promoter methylation status of ten putative (tumor suppressor) genes that are associated with $\mathrm{SHH}$, WNT signaling and (hair follicle) tumors in BCC and healthy control samples by methylation-specific PCR. In chapter 2.2 we aimed to expand the current understanding of BCC biology by combining genome-wide methylated DNA enrichment sequencing (MethylCap-seq) and RNA sequencing on primary BCCs and healthy skin controls. High-throughput next-generation sequencing can provide data about the genome, methylome and transciptome of malignancies and normal tissue of any organism. Chapter 2.3 examines a laBCC of the face. (I)aBCCs are tumors capable of deep tissue invasion, have higher metastasis rates and substantial morbidity and even mortality compared to common BCCs. ${ }^{74}$ Since (I)aBCCs are histologically indistinguishable from common BCCs, we looked for differences in methylation between this type of tumor and common BCCs, that would help to elucidate the origin of this relatively uncommon presentation. We discuss the results of genomewide MethylCap-seq and RNA-seq of a laBCC compared with regular BCCs and healthy skin.

The aim of chapter $\mathbf{3}$ is to probe hypoxia signaling in benign and malignant hair follicle tumors: trichoepithelioma (TE) and BCC. While the human dermis is well oxygenated and the epidermis is modestly hypoxic, the hair follicle is considered as a moderately to severely hypoxic microenvironment. ${ }^{121}$ Based on the hypoxia affecting the hair follicle and the known role of hypoxia related signaling in tumor growth ${ }^{122}$, we hypothesized that hypoxia response pathways are involved in the pathogenesis of TEs and BCCs. In this study, the results of immunohistochemical analysis of the most important target genes in the hypoxia response are presented. 


\section{Part two: new topical and systemic treatment modalities for BCC}

Chapter 4 comprises two parts. In chapter 4.1 we discuss the results of a single-blind, phase II, randomized controlled trial comparing topical diclofenac, calcitriol and a combination of both as treatment for sBCCs and nBCCs. Diclofenac and calcitriol are both associated with a reduction of tumor proliferation and induction of apoptosis. Also, diclofenac can inhibit WNT-signaling and the SHH signaling pathway can be inhibited by calcitriol. ${ }^{31,33,39,123}$ We aimed to test the hypothesis that treatment of sBCCs and nBCCs with diclofenac and/or calcitriol would lead to a histologic reduction of the marker Ki-67 (proliferation) and induction of BCL-2 (apoptosis). Chapter $\mathbf{4 . 2}$ discusses reproducibility of semi-automated quantification compared to manual quantification of two well-known immunohistochemical markers in sBCCs and nBCCs using a dedicated software program. In general, manual, pathologist-based quantification remains the current standard for $\mathrm{IHC}$ evaluation. ${ }^{124}$ However, a quantitative estimation of percent positivity is a subjective, pathologist-dependent approach and may demonstrate high intra- and inter-observer variability. ${ }^{125,126}$ Additionally, manual quantification is a time-consuming process. ${ }^{127}$ So therefore, the hypothesis underlying this study was that semi-automated quantification is a reproducible and time-efficient manner to analyze established immunohistochemical markers.

The aim of chapter $\mathbf{5}$ is to gain insight into the mechanisms underlying resistance against the orally active synthetic cyclopamine-like SMO-inhibitor vismodegib in patients with laBCCs. Vismodegib, a Hedgehog $(\mathrm{HH})$ pathway inhibitor, has been approved in 2012 by the US Food and Drug Administration (FDA) for the treatment of (I)aBCCs and mBCCs. ${ }^{128}$ Recent follow-up results of a phase II trial showed complete and partial response rates of $47.6 \%$ $((\mathrm{I}) \mathrm{aBCC})$ and $33.3 \%$ (mBCC) of patients, respectively. ${ }^{107}$ Initial response to vismodegib followed by secondary tumor progression has already been observed. 1,110-113 This chapter consists of two parts. In chapter $\mathbf{5 . 1}$ we describe a patient with a laBCC on the dorsal side of her left shoulder. She developed resistance to vismodegib after an initial complete clinical response. We hypothesized that SMO mutations may develop during vismodegib treatment, leading to secondary resistance. Mutational analysis of PTCH1 and SMO was performed on primary tumor, as well as on newly developed tumors and adjacent normal skin in an attempt to confirm our hypothesis. Chapter $\mathbf{5 . 2}$ describes a patient with a laBCC of the nose, right cheek and upper lip, who developed hyperkeratosis and comedo-like lesions after 7 weeks of vismodegib treatment. We hypothesized that the primary BCC had transformed into a SCC. Therefore, immunohistochemical analysis was performed to rule out this possibility.

Chapter $\mathbf{6}$ provides a general discussion and valorization. In chapter $\mathbf{7}$ a summary of the main findings of this thesis is given. 


\section{References}

1. Madan V, Lear JT, Szeimies RM. Non-melanoma skin cancer. Lancet 2010;375:673-85.

2. Tsao H, Atkins MB, Sober AJ. Management of cutaneous melanoma. The New England journal of medicine 2004;351:998-1012.

3. Snow SN, Sahl W, Lo JS, et al. Metastatic basal cell carcinoma. Report of five cases. Cancer 1994;73:328-35.

4. Zoccali G, Pajand R, Papa P, Orsini G, Lomartire N, Giuliani M. Giant basal cell carcinoma of the skin: literature review and personal experience. Journal of the European Academy of Dermatology and Venereology : JEADV 2012;26:942-52.

5. Flohil SC, Seubring I, van Rossum MM, Coebergh JW, de Vries E, Nijsten T. Trends in Basal Cell Carcinoma Incidence Rates: A 37-Year Dutch Observational Study. The Journal of investigative dermatology 2012

6. Lomas A, Leonardi-Bee J, Bath-Hextall F. A systematic review of worldwide incidence of nonmelanoma skin cancer. The British journal of dermatology 2012;166:1069-80.

7. Flohil SC, de Vries E, Neumann HA, Coebergh JW, Nijsten T. Incidence, prevalence and future trends of primary basal cell carcinoma in the Netherlands. Acta dermato-venereologica 2011;91:24-30.

8. Flohil SC, Koljenovic S, de Haas ER, Overbeek LI, de Vries E, Nijsten T. Cumulative risks and rates of subsequent basal cell carcinomas in the Netherlands. The British journal of dermatology 2011;165:87481.

9. Wu S, Han J, Laden F, Qureshi AA. Long-term ultraviolet flux, other potential risk factors, and skin cancer risk: a cohort study. Cancer Epidemiol Biomarkers Prev 2014;23:1080-9.

10. . at http://www.skincancer.org/prevention/tanning/tale-of-tanning.)

11. Richmond-Sinclair NM, Pandeya N, Ware RS, et al. Incidence of basal cell carcinoma multiplicity and detailed anatomic distribution: Iongitudinal study of an Australian population. The Journal of investigative dermatology 2009;129:323-8.

12. Staples MP, Elwood M, Burton RC, Williams JL, Marks R, Giles GG. Non-melanoma skin cancer in Australia: the 2002 national survey and trends since 1985. The Medical journal of Australia 2006;184:6-10.

13. Xiang F, Lucas R, Hales S, Neale R. Incidence of nonmelanoma skin cancer in relation to ambient UV radiation in white populations, 1978-2012: empirical relationships. JAMA dermatology 2014;150:1063-71.

14. Chen H, Weng QY, Fisher DE. UV signaling pathways within the skin. The Journal of investigative dermatology 2014;134:2080-5

15. Reichrath J, Rass K. Ultraviolet damage, DNA repair and vitamin D in nonmelanoma skin cancer and in malignant melanoma: an update. Advances in experimental medicine and biology 2014;810:208-33.

16. Greinert R, Volkmer B, Henning S, et al. UVA-induced DNA double-strand breaks result from the repair of clustered oxidative DNA damages. Nucleic acids research 2012;40:10263-73.

17. Roewert-Huber J, Lange-Asschenfeldt B, Stockfleth E, Kerl H. Epidemiology and aetiology of basal cell carcinoma. The British journal of dermatology 2007;157 Suppl 2:47-51.

18. Marzuka AG, Book SE. Basal Cell Carcinoma: Pathogenesis, Epidemiology, Clinical Features, Diagnosis, Histopathology, and Management. Yale J Biol Med 2015;88:167-79.

19. Kasper M, Jaks V, Hohl D, Toftgard R. Basal cell carcinoma - molecular biology and potential new therapies. The Journal of clinical investigation 2012;122:455-63.

20. Yang SH, Andl T, Grachtchouk V, et al. Pathological responses to oncogenic Hedgehog signaling in skin are dependent on canonical Wnt/beta3-catenin signaling. Nature genetics 2008;40:1130-5.

21. Youssef KK, Van Keymeulen A, Lapouge G, et al. Identification of the cell lineage at the origin of basal cell carcinoma. Nature cell biology 2010;12:299-305. 
Peterson SC, Eberl M, Vagnozzi AN, et al. Basal cell carcinoma preferentially arises from stem cells within hair follicle and mechanosensory niches. Cell Stem Cell 2015;16:400-12.

Doucet YS, Woo SH, Ruiz ME, Owens DM. The touch dome defines an epidermal niche specialized for mechanosensory signaling. Cell Rep 2013;3:1759-65.

Grachtchouk M, Pero J, Yang SH, et al. Basal cell carcinomas in mice arise from hair follicle stem cells and multiple epithelial progenitor populations. The Journal of clinical investigation 2011;121:1768-81.

Epstein EH. Basal cell carcinomas: attack of the hedgehog. Nature reviews Cancer 2008;8:743-54.

Rohatgi R, Scott MP. Patching the gaps in Hedgehog signalling. Nature cell biology 2007;9:1005-9.

Chiang C, Swan RZ, Grachtchouk M, et al. Essential role for Sonic hedgehog during hair follicle morphogenesis. Developmental biology 1999;205:1-9.

Reifenberger J, Wolter M, Knobbe CB, et al. Somatic mutations in the PTCH, SMOH, SUFUH and TP53 genes in sporadic basal cell carcinomas. The British journal of dermatology 2005;152:43-51.

Roop D, Toftgard R. Hedgehog in Wnterland. Nature genetics 2008;40:1040-1.

El-Bahrawy M, El-Masry N, Alison M, Poulsom R, Fallowfield M. Expression of beta-catenin in basal cell carcinoma. The British journal of dermatology 2003;148:964-70.

Barker N, Clevers H. Mining the Wnt pathway for cancer therapeutics. Nature reviews 2006;5:997-1014.

Takahashi-Yanaga F, Sasaguri T. The Wnt/beta-catenin signaling pathway as a target in drug discovery. J Pharmacol Sci 2007;104:293-302.

Fecker LF, Stockfleth E, Nindl I, Ulrich C, Forschner T, Eberle J. The role of apoptosis in therapy and prophylaxis of epithelial tumours by nonsteroidal anti-inflammatory drugs (NSAIDs). The British journal of dermatology 2007;156 Suppl 3:25-33.

Elmets CA, Ledet JJ, Athar M. Cyclooxygenases: mediators of UV-induced skin cancer and potential targets for prevention. The Journal of investigative dermatology 2014;134:2497-502.

Bank A, Yu J, Zhang L. NSAIDs downregulate Bcl-X(L) and dissociate BAX and Bcl-X(L) to induce apoptosis in colon cancer cells. Nutrition and cancer 2008;60 Suppl 1:98-103.

6. Tang JY, Aszterbaum M, Athar M, et al. Basal cell carcinoma chemoprevention with nonsteroidal anti-inflammatory drugs in genetically predisposed PTCH1+/- humans and mice. Cancer Prev Res (Phila) 2010;3:25-34.

7. Ulrich C, Johannsen A, Rowert-Huber J, Ulrich M, Sterry W, Stockfleth E. Results of a randomized, placebo-controlled safety and efficacy study of topical diclofenac 3\% gel in organ transplant patients with multiple actinic keratoses. European journal of dermatology : EJD 2010;20:482-8.

8ivers JK, Arlette J, Shear N, Guenther L, Carey W, Poulin Y. Topical treatment of actinic keratoses with $3.0 \%$ diclofenac in 2.5\% hyaluronan gel. The British journal of dermatology 2002;146:94-100.

Deeb KK, Trump DL, Johnson CS. Vitamin D signalling pathways in cancer: potential for anticancer therapeutics. Nature reviews Cancer 2007;7:684-700.

0. Bijlsma MF, Spek CA, Zivkovic D, van de Water S, Rezaee F, Peppelenbosch MP. Repression of smoothened by patched-dependent (pro-)vitamin D3 secretion. PLoS biology 2006;4:e232.

1. Tang JY, Xiao TZ, Oda Y, et al. Vitamin D3 inhibits hedgehog signaling and proliferation in murine Basal cell carcinomas. Cancer Prev Res (Phila) 2011;4:744-51.

Baylin SB. DNA methylation and gene silencing in cancer. Nat Clin Pract Oncol 2005;2 Suppl 1:S4-11.

Esteller M. Aberrant DNA methylation as a cancer-inducing mechanism. Annu Rev Pharmacol Toxicol 2005;45:629-56.

Herman JG, Baylin SB. Gene silencing in cancer in association with promoter hypermethylation. The New England journal of medicine 2003;349:2042-54.

Esteller M. Epigenetics in cancer. The New England journal of medicine 2008;358:1148-59. 
46. Goldberg M, Rummelt C, Laerm A, Helmbold P, Holbach LM, Ballhausen WG. Epigenetic silencing contributes to frequent loss of the fragile histidine triad tumour suppressor in basal cell carcinomas. The British journal of dermatology 2006;155:1154-8.

47. Heitzer E, Bambach I, Dandachi N, Horn M, Wolf P. PTCH promoter methylation at low level in sporadic basal cell carcinoma analysed by three different approaches. Experimental dermatology 2010;19:926-8.

48. Sathyanarayana UG, Moore AY, Li L, et al. Sun exposure related methylation in malignant and non-malignant skin lesions. Cancer letters 2007;245:112-20.

49. van Doorn R, Gruis NA, Willemze R, van der Velden PA, Tensen CP. Aberrant DNA methylation in cutaneous malignancies. Seminars in oncology 2005;32:479-87.

50. Wu W, Zhang J, Yang H, Shao Y, Yu B. Examination of AKAP12 promoter methylation in skin cancer using methylation-sensitive high-resolution melting analysis. Clinical and experimental dermatology 2011;36:381-5.

51. Konig S, Nitzki F, Uhmann A, et al. Depletion of cutaneous macrophages and dendritic cells promotes growth of basal cell carcinoma in mice. PloS one 2014;9:e93555.

52. Lesack K, Naugler C. Morphometric characteristics of basal cell carcinoma peritumoral stroma varies among basal cell carcinoma subtypes. BMC dermatology 2012;12:1.

53. Coussens LM, Werb Z. Inflammation and cancer. Nature 2002;420:860-7.

54. Carlson JA, Combates NJ, Stenn KS, Prouty SM. Anaplastic neoplasms arising from basal cellcarcinoma xenotransplants into SCID-beige mice. Journal of cutaneous pathology 2002;29:268-78.

55. Marzbani E, Inatsuka C, Lu H, Disis ML. The invisible arm of immunity in common cancer chemoprevention agents. Cancer Prev Res (Phila) 2013;6:764-73.

56. Berger A. Th1 and Th2 responses: what are they? BMJ 2000;321:424.

57. Yanofsky VR, Mitsui H, Felsen D, Carucci JA. Understanding dendritic cells and their role in cutaneous carcinoma and cancer immunotherapy. Clin Dev Immunol 2013;2013:624123.

58. Swetter SM, Boldrick JC, Pierre P, Wong P, Egbert BM. Effects of biopsy-induced wound healing on residual basal cell and squamous cell carcinomas: rate of tumor regression in excisional specimens. Journal of cutaneous pathology 2003;30:139-46.

59. Fujimura T, Kakizaki A, Kambayashi Y, Aiba S. Basal cell carcinoma with spontaneous regression: a case report and immunohistochemical study. Case Rep Dermatol 2012;4:125-32.

60. Kaporis HG, Guttman-Yassky E, Lowes MA, et al. Human basal cell carcinoma is associated with Foxp3+ T cells in a Th2 dominant microenvironment. The Journal of investigative dermatology 2007;127:2391-8.

61. Barr LH, Menard JW. Marjolin's ulcer. The LSU experience. Cancer 1983;52:173-5.

62. de Jager WC, Walbeehm ET, Wagner T, Gerritsen MJ. [Marjolin ulcer; malignant degeneration in a chronic wound]. Nederlands tijdschrift voor geneeskunde 2015;159:A8203.

63. Rippey JJ. Why classify basal cell carcinomas? Histopathology 1998;32:393-8.

64. Crowson AN. Basal cell carcinoma: biology, morphology and clinical implications. Modern pathology : an official journal of the United States and Canadian Academy of Pathology, Inc 2006;19 Suppl 2:S127-47.

65. Smeets NW, Kuijpers DI, Nelemans P, et al. Mohs' micrographic surgery for treatment of basal cell carcinoma of the face--results of a retrospective study and review of the literature. The British journal of dermatology 2004;151:141-7.

66. Telfer NR, Colver GB, Morton CA, British Association of D. Guidelines for the management of basal cell carcinoma. The British journal of dermatology 2008;159:35-48.

67. Evidenced-based guideline Treatment of the basal cell carcinoma. at http://www.nvpc.nl/uploads/stand/ 150414DOC-MB-Definitieve_richtlijn_Basaalcelcarcinoom_2014_goedgekeurd_ALV_14_april_2015154. pdf.) 
68. Roozeboom MH, Mosterd K, Winnepenninckx VJ, Nelemans PJ, Kelleners-Smeets NW. Agreement between histological subtype on punch biopsy and surgical excision in primary basal cell carcinoma. Journal of the European Academy of Dermatology and Venereology : JEADV 2013;27:894-8.

69. Weedon D. Skin Pathology. 3rd ed. Tokyo: Churchill Livingstone; 2009.

70. Menzies SW, Westerhoff K, Rabinovitz H, Kopf AW, McCarthy WH, Katz B. Surface microscopy of pigmented basal cell carcinoma. Archives of dermatology 2000;136:1012-6.

71. Betti R, Radaelli G, Mussino F, Menni S, Crosti C. Anatomic location and histopathologic subtype of basal cell carcinomas in adults younger than 40 or 90 and older: any difference? Dermatol Surg 2009;35:201-6.

72. Edge SB BD, Compton CC, Fritz AG, Greene FL, Trotti A AJCC Cancer Staging Manual 7ed: Springer: New York; 2010.

73. Lear JT, Corner C, Dziewulski $\mathrm{P}$, et al. Challenges and new horizons in the management of advanced basal cell carcinoma: a UK perspective. British journal of cancer 2014;111:1476-81.

74. Archontaki M, Stavrianos SD, Korkolis DP, et al. Giant Basal cell carcinoma: clinicopathological analysis of 51 cases and review of the literature. Anticancer research 2009;29:2655-63.

75. Caccialanza M, Percivalle S, Piccinno R. Possibility of treating basal cell carcinomas of nevoid basal cell carcinoma syndrome with superficial X-ray therapy. Dermatology 2004;208:60-3.

76. Moeholt K, Aagaard H, Pfeiffer P, Hansen O. Platinum-based cytotoxic therapy in basal cell carcinoma--a review of the literature. Acta oncologica 1996;35:677-82.

77. Basset-Seguin N, Sharpe HJ, de Sauvage FJ. Efficacy of Hedgehog Pathway Inhibitors in Basal Cell Carcinoma. Molecular cancer therapeutics 2015;14:633-41.

78. Walling HW, Fosko SW, Geraminejad PA, Whitaker DC, Arpey CJ. Aggressive basal cell carcinoma: presentation, pathogenesis, and management. Cancer metastasis reviews 2004;23:389-402.

79. Avril MF, Auperin A, Margulis A, et al. Basal cell carcinoma of the face: surgery or radiotherapy? Results of a randomized study. British journal of cancer 1997;76:100-6.

80. Narayanan K, Hadid OH, Barnes EA. Mohs micrographic surgery versus surgical excision for periocular basal cell carcinoma. Cochrane database of systematic reviews (Online) 2014;12:CD007041.

81. Rhodes LE, De Rie MA, Leifsdottir R, et al. Five-year follow-up of a randomized, prospective trial of topical methyl aminolevulinate photodynamic therapy vs surgery for nodular basal cell carcinoma. Archives of dermatology 2007; 143:1131-6.

82. Rowe DE, Carroll RJ, Day CL, Jr. Mohs surgery is the treatment of choice for recurrent (previously treated) basal cell carcinoma. The Journal of dermatologic surgery and oncology 1989;15:424-31.

83. Rowe DE, Carroll RJ, Day CL, Jr. Long-term recurrence rates in previously untreated (primary) basal cell carcinoma: implications for patient follow-up. The Journal of dermatologic surgery and oncology 1989;15:315-28.

84. Mosterd K, Krekels GA, Nieman FH, et al. Surgical excision versus Mohs' micrographic surgery for primary and recurrent basal-cell carcinoma of the face: a prospective randomised controlled trial with 5-years' follow-up. The lancet oncology 2008;9:1149-56.

85. van Loo E, Mosterd K, Krekels GA, et al. Surgical excision versus Mohs' micrographic surgery for basal cell carcinoma of the face: A randomised clinical trial with 10 year follow-up. Eur J Cancer 2014;50:301120.

86. Moehrle M, Breuninger $\mathrm{H}$, Rocken M. A confusing world: what to call histology of three-dimensional tumour margins? Journal of the European Academy of Dermatology and Venereology : JEADV 2007;21:591-5.

87. Smeets NW, Krekels GA, Ostertag JU, et al. Surgical excision vs Mohs' micrographic surgery for basal-cell carcinoma of the face: randomised controlled trial. Lancet 2004;364:1766-72.

88. Macfarlane L, Waters A, Evans A, Affleck A, Fleming C. Seven years' experience of Mohs micrographic surgery in a UK centre, and development of a UK minimum dataset and audit standards. Clinical and experimental dermatology 2013;38:262-9. 
89. Cho M, Gordon L, Rembielak A, Woo TC. Utility of radiotherapy for treatment of basal cell carcinoma: a review. The British journal of dermatology 2014;171:968-73.

90. Hall VL, Leppard BJ, McGill J, Kesseler ME, White JE, Goodwin P. Treatment of basal-cell carcinoma: comparison of radiotherapy and cryotherapy. Clinical radiology 1986;37:33-4.

91. Caccialanza M, Piccinno R, Moretti D, Rozza M. Radiotherapy of carcinomas of the skin overlying the cartilage of the nose: results in 405 lesions. European journal of dermatology : EJD 2003;13:462-5.

92. Guix B, Finestres F, Tello J, et al. Treatment of skin carcinomas of the face by high-dose-rate brachytherapy and custom-made surface molds. International journal of radiation oncology, biology, physics 2000;47:95-102.

93. Silverman MK, Kopf AW, Gladstein AH, Bart RS, Grin CM, Levenstein MJ. Recurrence rates of treated basal cell carcinomas. Part 4: X-ray therapy. The Journal of dermatologic surgery and oncology 1992;18:549-54.

94. Arits $\mathrm{AH}$, Mosterd K, Essers BA, et al. Photodynamic therapy versus topical imiquimod versus topical fluorouracil for treatment of superficial basal-cell carcinoma: a single blind, non-inferiority, randomised controlled trial. The lancet oncology 2013;14:647-54.

95. Roozeboom MH, Arits AH, Nelemans PJ, Kelleners-Smeets NW. Overall treatment success after treatment of primary superficial basal cell carcinoma: a systematic review and meta-analysis of randomized and nonrandomized trials. The British journal of dermatology 2012;167:733-56.

96. Bath-Hextall F, Ozolins M, Armstrong SJ, et al. Surgical excision versus imiquimod 5\% cream for nodular and superficial basal-cell carcinoma (SINS): a multicentre, non-inferiority, randomised controlled trial. The lancet oncology 2014;15:96-105.

97. Vanneman M, Dranoff G. Combining immunotherapy and targeted therapies in cancer treatment. Nature reviews Cancer 2012;12:237-51.

98. Binns W, James LF, Shupe JL, Thacker EJ. Cyclopian-type malformation in lambs. Arch Environ Health 1962;5:106-8.

99. Keeler RF, Binns W. Teratogenic compounds of Veratrum californicum (Durand). V. Comparison of cyclopian effects of steroidal alkaloids from the plant and structurally related compounds from other sources. Teratology 1968;1:5-10.

100. Chen JK, Taipale J, Cooper MK, Beachy PA. Inhibition of Hedgehog signaling by direct binding of cyclopamine to Smoothened. Genes \& development 2002;16:2743-8.

101. Taipale J, Chen JK, Cooper MK, et al. Effects of oncogenic mutations in Smoothened and Patched can be reversed by cyclopamine. Nature 2000;406:1005-9.

102. Abidi A. Hedgehog signaling pathway: a novel target for cancer therapy: vismodegib, a promising therapeutic option in treatment of basal cell carcinomas. Indian J Pharmacol 2014;46:3-12.

103. Liu LS, Colegio OR. Molecularly targeted therapies for nonmelanoma skin cancers. International journal of dermatology 2013;52:654-65.

104. Yang L, Xie G, Fan Q, Xie J. Activation of the hedgehog-signaling pathway in human cancer and the clinical implications. Oncogene;29:469-81.

105. Axelson M, Liu K, Jiang X, et al. U.S. Food and Drug Administration approval: vismodegib for recurrent, locally advanced, or metastatic basal cell carcinoma. Clinical cancer research : an official journal of the American Association for Cancer Research 2013;19:2289-93.

106. Sekulic A, Migden MR, Oro AE, et al. Efficacy and safety of vismodegib in advanced basal-cell carcinoma. The New England journal of medicine 2012;366:2171-9.

107. Erivedge SmPC, June 2014.

108. Basset-Seguin N, Hauschild A, Grob JJ, et al. Vismodegib in patients with advanced basal cell carcinoma (STEVIE): a pre-planned interim analysis of an international, open-label trial. The lancet oncology 2015;16:729-36. 
109. Sekulic A, Mangold AR, Northfelt DW, LoRusso PM. Advanced basal cell carcinoma of the skin: targeting the hedgehog pathway. Current opinion in oncology 2013;25:218-23.

110. Chang AL, Atwood SX, Tartar DM, Oro AE. Surgical excision after neoadjuvant therapy with vismodegib for a locally advanced basal cell carcinoma and resistant basal carcinomas in Gorlin syndrome. JAMA dermatology 2013;149:639-41.

111. Chang AL, Oro AE. Initial assessment of tumor regrowth after vismodegib in advanced Basal cell carcinoma. Archives of dermatology 2012;148:1324-5.

112. Dijkgraaf GJ, Alicke B, Weinmann L, et al. Small molecule inhibition of GDC-0449 refractory smoothened mutants and downstream mechanisms of drug resistance. Cancer research 2011;71:435-44.

113. Fecher LA. Systemic therapy for inoperable and metastatic basal cell cancer. Current treatment options in oncology 2013;14:237-48.

114. Yauch RL, Dijkgraaf GJ, Alicke B, et al. Smoothened mutation confers resistance to a Hedgehog pathway inhibitor in medulloblastoma. Science 2009;326:572-4.

115. Atwood SX, Sarin KY, Whitson RJ, et al. Smoothened variants explain the majority of drug resistance in basal cell carcinoma. Cancer cell 2015;27:342-53.

116. Hatziapostolou M, lliopoulos D. Epigenetic aberrations during oncogenesis. Cellular and molecular life sciences : CMLS 2011;68:1681-702.

117. Bath-Hextall F, Ozolins M, Armstrong SJ, et al. Surgical excision versus imiquimod 5\% cream for nodular and superficial basal-cell carcinoma (SINS): a multicentre, non-inferiority, randomised controlled trial. The lancet oncology 2014;15:96-105.

118. Brechbiel J, Miller-Moslin K, Adjei AA. Crosstalk between hedgehog and other signaling pathways as a basis for combination therapies in cancer. Cancer treatment reviews 2014.

119. Schuebel KE, Chen W, Cope L, et al. Comparing the DNA hypermethylome with gene mutations in human colorectal cancer. PLoS genetics 2007;3:1709-23.

120. Giacinti L, Vici P, Lopez M. Epigenome: a new target in cancer therapy. La Clinica terapeutica 2008;159:347-60.

121. Evans SM, Schrlau AE, Chalian AA, Zhang P, Koch CJ. Oxygen levels in normal and previously irradiated human skin as assessed by EF5 binding. The Journal of investigative dermatology 2006;126:2596-606.

122. Wouters BG, Koritzinsky M. Hypoxia signalling through mTOR and the unfolded protein response in cancer. Nature reviews Cancer 2008;8:851-64.

123. Tjiu JW, Liao YH, Lin SJ, et al. Cyclooxygenase-2 overexpression in human basal cell carcinoma cell line increases antiapoptosis, angiogenesis, and tumorigenesis. The Journal of investigative dermatology 2006;126:1143-51.

124. Camp RL, Chung GG, Rimm DL. Automated subcellular localization and quantification of protein expression in tissue microarrays. Nature medicine 2002;8:1323-7.

125. Nagamalini BR, Suneela S, Narayan TV, et al. Origin of ameloblastoma from Basal cells of the oral epithelium- establishing the relation using neuroectodermal markers. Journal of clinical and diagnostic research : JCDR 2014;8:ZC44-7.

126. De Meyer T, Mampaey E, Vlemmix M, et al. Quality evaluation of methyl binding domain based kits for enrichment DNA-methylation sequencing. PloS one 2013;8:e59068.

127. Daniel K, Maria A, Amelie L, et al. Somatostatin receptor immunohistochemistry in neuroendocrine tumors: comparison between manual and automated evaluation. International journal of clinical and experimental pathology 2014;7:4971-80.

128. Kato N, Endo Y, Tamura G, Motoyama T. Ameloblastoma with basal cell carcinoma-like feature emerging as a nasal polyp. Pathology international 1999;49:747-51. 

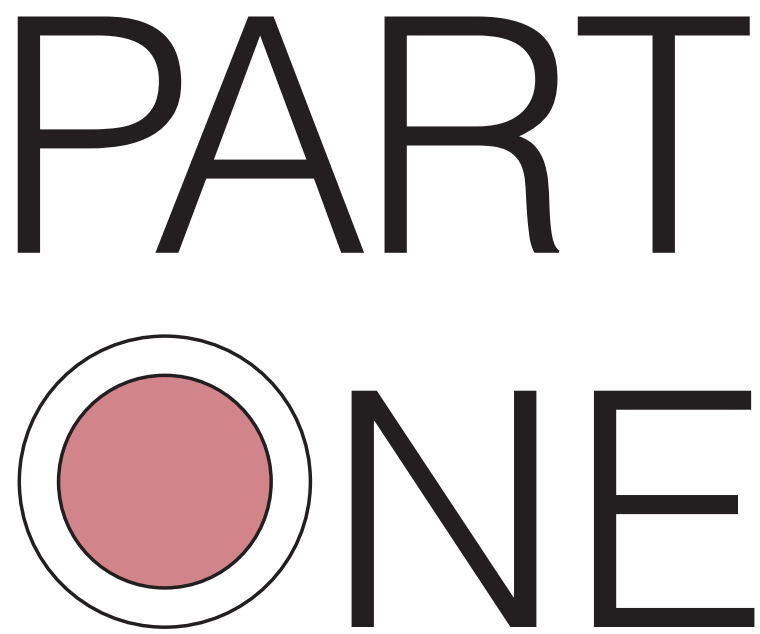

PATHOGENESIS OF BASAL CELL CARCINOMA 


\section{Chapter 2.1}

Epigenetic changes in basal cell carcinoma effect SHH and WNT signaling components

Brinkhuizen T, van den Hurk K, Winnepenninckx VJ, de Hoon JP, van Marion AM, Veeck J, van Engeland M, van Steensel MAM.

PLoS One. 2012;7(12):e51710. 


\begin{abstract}
Background: The genetic background of Basal Cell Carcinoma (BCC) has been studied extensively, while its epigenetic makeup has received comparatively little attention. Epigenetic alterations such as promoter hypermethylation silence tumor suppressor genes (TSG) in several malignancies.
\end{abstract}

Objective:We sought to analyze the promoter methylation status of ten putative (tumor suppressor) genes that are associated with Sonic Hedgehog (SHH), WNT signaling and (hair follicle) tumors in a large series of $112 \mathrm{BCC}$ and 124 healthy control samples by methylation-specific PCR.

Results: Gene promoters of $\mathrm{SHH}(\mathrm{P}=0.016)$, adenomatous polyposis coli $(A P C)(\mathrm{P}=0.003)$, secreted frizzled-related protein 5 (SFRP5) $(\mathrm{P}=0.004)$ and Ras association domain family $1 \mathrm{~A}(R A S S F 1 A)(P=0.023)$ showed significantly more methylation in $B C C$ versus normal skin. mRNA levels of these four genes were reduced for APC and SFRP5 in BCC $(n=6)$ vs. normal skin $(n=6)$. Down regulation of SHH, APC and RASSF1A could be confirmed on protein level as well ( $P<0.001$ for all genes) by immunohistochemical staining. Increased canonical WNT activity was visualized by $\beta$-catenin staining, showing nuclear $\beta$-catenin in only $28 / 101$ (27.7\%) of BCC. Absence of nuclear $\beta$-catenin in some samples may be due to high levels of membranous E-cadherin (in $94.1 \%$ of the samples).

Conclusions: We provide evidence that promoter hypermethylation of key players within the $\mathrm{SHH}$ and WNT pathways is frequent in BCC, consistent with their known constitutive activation in BCC. Epigenetic gene silencing putatively contributes to BCC tumorigenesis, indicating new venues for treatment. 


\section{Introduction}

Basal cell carcinoma (BCC) accounts for $75 \%$ of all skin cancers and is the most frequent malignancy in Caucasians. Its incidence is rising by $3-8 \%$ each year ${ }^{1}$, resulting in an average lifetime risk for Caucasians of developing BCC of $30 \%,{ }^{2,3}$ BCC rarely metastasize ${ }^{4}$, but when left untreated they may cause extensive local tissue destruction. ${ }^{4,5}$ Surgical excision is the current standard treatment, with average costs in the Netherlands of $€ 900$ per procedure, amounting to a total of $€ 45$ million by 2015.6,7 The inevitably rising workload can be expected to stress the health care system even further. Hence, BCC is becoming a serious health problem. There is a clear need for a simple and cost-efficient medical treatment. In order to develop one, a thorough understanding of BCC pathobiology will be required and the past few years have witnessed considerable progress in this respect.

Inappropriate activity of the Sonic Hedgehog (SHH) pathway due to mutations of its component genes is firmly implicated in BCC pathogenesis. Inactivating mutations of Patched-1 (PTCH1) are the most common (30-60\%) in sporadic BCC ${ }^{8-11}$, followed by mutations (10$20 \%$ ) in Smoothened (SMO) or Suppressor of Fused (SU(FU)). ${ }^{11-16}$ Even though mutations of the SHH target gene, Glioma-associated oncogene homolog-1 (GLI1), are uncommon, approximately $90 \%$ of sporadic BCC overexpress GLI1, which contributes to tumor growth. ${ }^{17}$ In addition, Yang and colleagues proved the wingless-type MMTV integration site family (WNT) pathway to be essential in tumorigenic response to deregulated $\mathrm{SHH}$ signaling, suggesting crosstalk between the SHH and canonical WNT pathways in BCC. ${ }^{18}$ Non-canonical WNT signaling, however, might also be active in the context of repressed canonical WNT signaling in BCC, as recently reported by Pourreyron et al. ${ }^{19}$ Thus, both WNT signaling pathways might act in a mutually antagonistic fashion in driving BCC growth. In all, the genetic aspects of BCC development seem to be well defined. However, most if not all cancers are characterized by epigenetic alterations in addition to genetic changes.20 Epigenetic modifications are heritable changes in deoxyribonucleic acid (DNA) structure other than alterations in the DNA sequence, and when pathologically altered are capable of driving malignant tumor development and progression. To date, DNA hypermethylation is the best-characterized epigenetic mechanism, comprising the addition of a methyl group to a cytosine base adjacent to a guanine base (the CG dinucleotide). ${ }^{20} \mathrm{CG}$-rich areas (CpG islands) located in the promoter region of many genes become hypermethylated in numerous malignancies ${ }^{21}$ leading to gene silencing. ${ }^{22}$ Promoter hypermethylation can precede genetic mutations and genomic instability in tumor development, and may thus not only be crucial for carcinogenesis, but also represent a potential therapeutic target. ${ }^{23}$ Indeed, DNA demethylating agents, such as 5-azacytidine (Vidaza ${ }^{\circledR}$, Celgene) and 5-aza-2'-deoxycytidine (Dacogen ${ }^{\mathrm{TM}}$, MGI Pharma) can lead to reactivation of silenced genes. ${ }^{24}$ Thus, it would be of interest to determine the contribution of promoter hypermethylation to BCC pathogenesis. To date, a very restricted number of studies have addressed this question in a limited number of samples. ${ }^{25-30}$ Therefore, we decided to assess the promoter CpG island methylation status of nine tumor suppressor genes (TSGs). Patched Homolog 1 (PTCH1) is the $\mathrm{SHH}$ receptor and is the most commonly mutated tumor suppressor in BCC, whereas adenomatous polyposis coli 
(APC), secreted frizzled-related protein 1 (SFRP1), SFRP2, SFRP4, and SFRP5 are all negative regulators of the canonical WNT pathway. Sustained signaling through the canonical WNT pathway contributes to the development of colorectal cancer as well as basal cell carcinoma. ${ }^{18,19,31}$ Since BCC are considered as hair follicle tumors, we additionally analyzed the cylindromatosis (CYLD) and tuberous sclerosis-1 (TSC1) genes, which both are TSGs known to be involved in classic hair follicle tumor syndromes. ${ }^{32,33}$ Additionally, TSC complex proteins are crucial negative regulators of mTOR activity, which is implicated in tumor growth. ${ }^{34}$ Ras association domain family 1 A (RASSF1A) is a well-known TSG, promoter methylation of which has been described as an early and frequent event in several malignancies including basal cell carcinoma. ${ }^{28,35}$ Finally we examined $\mathrm{SHH}$, since we hypothesized that downstream activation of its signaling pathway would permit it to become inactivated through methylation.

We have demonstrated significant hypermethylation affecting several of the selected genes in $\mathrm{BCC}$, supporting the involvement of epigenetic aberrations in the most common skin cancer. 


\section{Materials and Methods}

\section{Primary tissue specimens}

The methylation status of ten selected genes was examined in 112 sporadic BCC samples (107 formalin-fixed paraffin-embedded (FFPE), five fresh frozen (FF)) diagnosed at the Department of Dermatology, MUMC+. All BCC samples were obtained from the Maastricht Pathology Tissue Collection (MPTC). Distinct histological subtypes included were: superficial (sBCC) $(n=37)$, nodular (nBCC) $(n=53)$, and infiltrative (iBCC) $(n=22)$. Thirty of these samples were used for immunohistochemical analysis of the expression of $\mathrm{SHH}, \mathrm{APC}$ and RASSF1A. $\beta$-catenin and E-cadherin were assessed on respectively 101 (30 sBCC, $40 \mathrm{nBCC}, 31 \mathrm{iBCC}$ ) and 59 BCC (17 sBCC, 27 nBCC, 15 iBCC) independent samples. Patients included 58 men (mean age 68.1 years, $S D \pm 11.0$ ) and 54 women (mean age 66.2 years, $S D \pm 15.1$ ) A total of 124 healthy control tissue samples (71 FFPE, 53 FF) was collected at autopsies and matched with the 112 BCC patients with regard to age and gender (Table 1). Relatives gave their written consent for usage of the skin tissues. For 31 of these controls, no data were available concerning age and gender. Collection, storage and use of all tissues and patient data were performed in agreement with the "Code for Proper Secondary Use of Human Tissue in the Netherlands". All of the used samples and corresponding data were de-linked and anonymized. Usage of both BCC and control tissue samples was approved by the MPTC scientific committee (MPTC 2009-05).

\section{DNA isolation}

From each paraffin tissue block and FF sample, a $4 \mu \mathrm{m}$ section was cut and stained with haematoxylin \& eosin (H\&E) as a part of the routine intake procedure. All H\&E sections were reviewed by a dermato-pathologist (AM or VW) to confirm diagnosis and histological subtype. After deparaffinization of five sections ( $20 \mu \mathrm{m}$ ) of each FFPE sample, tumor tissue was macroscopically scraped and collected in $1.5 \mathrm{ml}$ tubes. The sections of theFF samples were directly collected into $1.5 \mathrm{ml}$ tubes. Genomic DNA was extracted from FFPE tissue sections by using a Qiagen microkit for DNA isolation (Qiagen, Venlo, The Netherlands) following the manufacturer's directions.

\section{Bisulfite modification of genomic DNA, methylation-specific PCR and bisulfite genomic sequencing}

Promoter $\mathrm{CpG}$ island methylation was determined by bisulfite genomic sequencing (BGS) for SHH, and by MSP for PTCH1, SHH, APC, SFRP1, SFRP2, SFRP4, SFRP5, CYLD, TSC1, and RASSF1A. Sodium bisulfite modification was performed on 500 ng of genomic DNA isolated from the tissue sections by use of an EZ DNA methylation kit (Zymo Research Co, Orange, CA, USA) according to the manufacturer's instructions. The modified DNA was eluted to $50 \mathrm{ng} / \mu \mathrm{l}$ in $\mathrm{H}_{2} \mathrm{O}$ and stored at $-80^{\circ} \mathrm{C}$. Nested methylation specific polymerase chain reaction (MSP) was performed on bisulfite-modified genomic DNA with primers specific for methylated DNA and unmethylated DNA as previously described ${ }^{36-38}$. Nested MSP is exquisitely suitable for methylation analysis of FFPE DNA 
since it is highly sensitive. Primer sequences and PCR conditions are listed in Table S1. To assess reproducibility of the nested MSP approach, MSP reactions were performed in duplicate starting from DNA amplification with flanking polymerase chain reaction (PCR) primers.

Table 1. Sample characteristics

Tumor $(n=112)$

Normal skin $(n=124)$

$\begin{array}{lrr}\text { Sex } & 58(51.3) & 50(53.8) \\ \text { Male, n (\%) } & 54(48.2) & 43(46.2) \\ \text { Female. } \mathrm{n}(\%) & 0(0) & 31(25.0) \\ \text { Unknown, } \mathrm{n}(\%) & \end{array}$

$\begin{array}{lll}\text { Age, years mean } & & \\ \text { Overall } & 67.2( \pm 13.1 \mathrm{SD}) & 68.1( \pm 14.0 \mathrm{SD}) \\ \text { Man } & 68.1( \pm 11.0 \mathrm{SD}) & 67.9( \pm 12.2 \mathrm{SD}) \\ \text { Women } & 66.2( \pm 15.1 \mathrm{SD}) & 68.4( \pm 16.0 \mathrm{SD})\end{array}$

\begin{tabular}{lrr} 
Tissue Source & & \\
\hline FFPE, $n(\%)$ & $107(95.5)$ & $71(57.3)$ \\
FF, $n(\%)$ & $5(4.5)$ & $53(42.7)$
\end{tabular}

$\begin{array}{ll}\text { BCC subtypes, } n(\%) & \\ \text { Superficial } & 37(32.7 \%) \\ \text { Nodular } & 53(46.9 \%) \\ \text { Infiltrative } & 22(19.6 \%)\end{array}$

${ }^{\star} \mathrm{FFPE}=$ Formalin-fixed, paraffin-embedded, $F F=$ Fresh- frozen

Non-concordant MSP results were analyzed a third time and concordance in two out of three assays was accepted as end result. The overall reproducibility for the MSP data was $90.3 \%$. For BGS analysis, one $\mu$ l of PCR product from bisulfite-modified genomic DNA was cloned into TOP10 bacteria using a TOPO-TA cloning kit (Invitrogen, Breda, The Netherlands). Bacteria were cultured at $37^{\circ} \mathrm{C}$ overnight. DNA was extracted from at least ten independent bacterial clones and sequenced using an automated DNA sequencer (Applied Biosystems, Foster City, CA, USA). Sequencing data was analyzed using Sequence Scanner v1.0 software (Applied Biosystems). Mean values were calculated and represented in pie-chart figures per CpG. 
RNA isolation, reverse transcription and quantitative reverse transcription PCR (RT-PCR)

Total ribonucleic acid (RNA) from FF samples containing more than 75\% BCC cells $(n=6)$ as evaluated from H\&E stained sections and FF normal skin samples $(n=6)$ was isolated using the standard procedure for TRIzol® RNA extraction (Invitrogen) and stored at $-80^{\circ} \mathrm{C}$. Complementary DNA (cDNA) synthesis was performed using the iScriptTM cDNA Synthesis kit (Biorad, Veenendaal, The Netherlands) according to the manufacturer's instructions. Quantitative RT-PCR on 30ng cDNA was carried out using primer sets for APC, RASSF1A, SFRP5, and SHH. Primer sequences and PCR conditions are listed in Table S1. Messenger RNA (mRNA) for SHH and RASSF1A could not be detected. APC and SFRP5 expression levels for each BCC sample were normalized to the housekeeping gene Cyclophylin $A$ and average expression levels in normal skin tissues $(n=6)$ by $2-\Delta \Delta \mathrm{Ct}$ parameter ${ }^{39}$. To assure accuracy, all reactions were performed in triplicate.

\section{Immunohistochemistry}

For SHH, APC and RASSF1A analysis, FFPE sections (4 $\mu \mathrm{m})$ were deparaffinized in xylene, rehydrated and incubated in $0.3 \%$ hydrogen peroxide $\left(\mathrm{H}_{2} \mathrm{O}_{2}\right)$ in methanol for 30 minutes to inactivate endogenous peroxidase activity. Antigen retrieval was performed by microwave treatment at $90 \mathrm{~W}$ for 10 minutes in $10 \mathrm{mM}$ citrate buffer ( $\mathrm{pH} 6$ ) (APC and SHH) or Envision Flex target retrieval solution high pH (Dako, Heverlee, Belgium) (RASSF1A). Next, non-specific protein binding was blocked using 3\% bovine-serum-albumin (BSA). Subsequently, the sections were incubated for $1 \mathrm{~h}$ at room temperature with primary antibodies listed in table S2. A horseradish peroxidase (HRP)-conjugated second antibody, either PowerVision+ (ImmunoVision Technology, Brisbane, CA, USA) (APC and SHH) or Envision detection system (Dako) (RASSF1A) was applied for 30 minutes. Bound antibody was visualized by using 3,3-diaminobenzidine (DAB) for 10 minutes. Tissue was counterstained with haematoxylin, dehydrated and sealed with coverslips. Phosphate-buffered saline (PBS) was used throughout for washing steps. For $\beta$-catenin and E-cadherin analysis, sections were pre-treated in a pre-treatment module using EnVision FLEX Target Retrieval Solution, High pH (Dako). Staining was performed on a Dako autostainer system using the Dako Envision Flex kit (K8002) for secondary detection. Sections were counterstained with Gill II haematoxylin, dehydrated and sealed with coverslips. For all antibodies, tissue known to be strongly expressing respective protein was included as positive control (Table S2). Negative controls (omission of the primary antibody) were included in all experiments.

\section{Interpretation of staining}

A specialized dermato-pathologist of the Department of Pathology, MUMC+ (VW) and an experienced resident of the Department of Dermatology, MUMC+ (TB), examined sections independently. Any discrepancy between the observers was discussed and resolved by consensus. $\beta$-catenin staining was assessed with respect to localization (membranous, cytoplasmic, and nuclear). For the other antibodies, the percentage positive tumor cells were determined by assessing ten randomly chosen high-power-fields (magnification 200x) per 
slide and the average of both observers' values was used for analysis. Additionally, the intensity of E-cadherin was considered with respect to an internal positive control ranging from 0 (no staining) to 1 (weak), 2 (moderate), and 3 (strong) staining.

\section{Statistics}

Statistical analyses were carried out using SPSS version 18.0 software (SPSS, Chicago, IL, USA). Discrete data were analyzed using a Chi-square or Fisher's exact test, where the independent-samples T-test was used for continuous variables. The correlation between two discrete variables was evaluated by the Cohen's kappa. ${ }^{40}$ To evaluate the effect of methylation on the probability of the presence of tumor, multivariate binary logistic regression analyses were performed with presence or absence of tumor as dependent variable. The two way random effect model with absolute agreement intra-class correlation coefficient (ICC) was used as inter-rater reliability analysis to determine consistency among raters. ${ }^{41}$ An ICC $\geq 0.75$ indicates excellent reproducibility. ${ }^{42}$ All reported $P$ values are two-sided, and $P$ values $\leq 0.05$ were considered statistically significant.

\section{Results}

\section{Methylation of SHH and WNT pathway components in BCC}

A total of nine candidate TSGs and one oncogene was examined in a series of primary BCC $(n=112)$ of three different subtypes and normal skin $(n=124)$. Characteristics of the tumor and normal skin samples are listed in Table 1. Primers located in promoter CpG islands were previously determined. ${ }^{36-38}$ Our data showed that promoters of four genes were significantly more frequently methylated in BCC tissue versus normal skin: $S H H(44 / 100(40.0 \%)$ vs. 30/122 (24.6\%), $P=0.016), A P C(64 / 110$ (58.2\%) vs. 47/124 (37.9\%), $P=0.003)$, SFRP5 (52/109 (47.7\%) vs. 28/100 (28.0\%), $P=0.004)$, and RASSF1A (52/112 (46.4\%) vs.. 39/124 (31.3\%), $P=0.023$. The correlation between methylation in SHH and SFRP5 was moderate (kappa 0.68 (95\% Cl 0.50-0.78)); no further correlations between genes could be detected. After mutual correction of the two genes SHH and SFRP5 by multivariate logistic regression, odds ratios for individual genes were still larger than 1 (O.R. $1.42(P=0.304)$ and 2.01 $(P=0.032)$ respectively), suggesting that methylation of SFRP5 is significantly associated with the occurrence of BCC. Notably, of the four significant differentially methylated genes, $\mathrm{nBCC}$ harbored more frequent methylation for APC and RASSF1A, whereas SHH and SFRP5 were more frequently methylated in iBCC. Nevertheless, none of these differences were significant because of the low sample numbers. Moreover, although SFRP4 overall was not significantly hypermethylated in BCC, its methylation frequencies did vary according to the subtype ( $P=0.010$ for sBCC vs. nBCC plus iBCC, $P=0.011$ sBCC vs. nBCC) (data not shown). No significant differential methylation could be detected for PTCH1, SFRP1, SFRP2, SFRP4, CYLD, and TSC1 (Figure 1A and 1B). When we compared sun-exposed (SE) ( $n=47)$ with sun-protected (SP) ( $n=54)$ normal skin tissue, only methylation of SFRP2 was found to be significantly different, with more frequent methylation in SP skin (SE 2.2\% vs.. SP $15.1 \%$, $P=0.035$ ) (Figure 1C). Gender or age-associated methylation patterns were not identified. 
As MSP primers of APC, SFRP5, and RASSF1A had been previously established and validated $^{38,43,44}$, we only validated our MSP results of the fourth differentially methylated gene, $S H H$, by BGS, which is considered the gold standard. ${ }^{45}$ Figure $1 \mathrm{D}$ depicts the BGS results of one methylated and one unmethylated SHH sample as measured by MSP, and BGS could confirm MSP results.

A

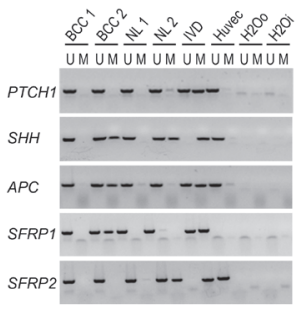

C

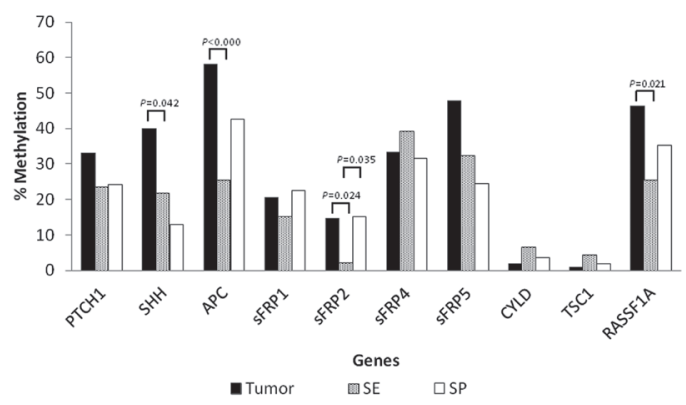

B

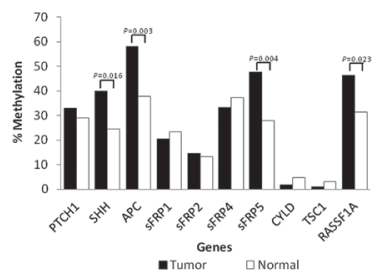

D

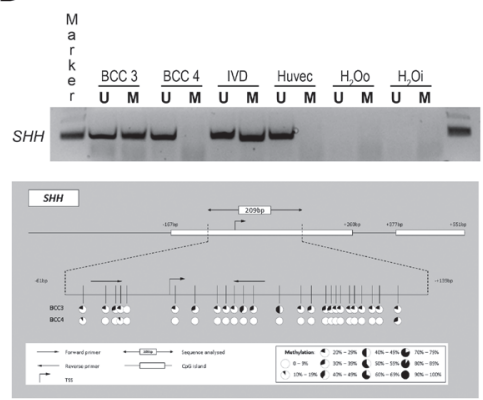

Figure 1. Methylation analysis in BCC and normal skin of ten candidate genes.

A. Illustration of MSP results of ten candidate genes resolved on a 2\% agarose gel. BCC: Basal cell carcinoma; NL, normal; IVD, In Vitro Methylated-DNA; Huvec, Human Umbilical Vein Endothelial Cells; U, unmethylated; M, methylated; $\mathrm{H} 20 \mathrm{O}$, water control outside PCR; H2Oi, water control inside PCR. B. Illustration of methylation frequencies of ten candidate genes in BCC $(n=112)$ and normal skin samples $(n=124)$. P-values represent the difference between percentage methylation in BCC an normal skin. C. Illustration of methylation frequencies of ten candidate genes in BCC, sun-exposed (SE) normal skin and sun-protected (SP) normal skin. P-values represent both the difference between percentage methylation in BCC and SE and SP. D. SHH sequence data of bisulfite treated genomic DNA from patients. Upper part shows the SHH promoter region starting 1000 base pairs (bp) upstream of the transcription start site (TSS) to 1000 bp downstream. White boxes indicate putative CpG islands (EMBOSS, http://emboss.sourceforge.net). The $256 \mathrm{bp}$ region sequenced stretches from $-51 \mathrm{bp}$ from the TSS to $+409 \mathrm{bp}$. Indicated with arrows are the forward and reverse methylation specific PCR (MSP) primers. Vertical bars represent CpG dinucleotides and pie -charts represent the percentage of methylated $\mathrm{CpG}$ sites (percentage over at least 10 sequenced clones). 
Expression of SHH, APC, SFRP5, and RASSF1A is reduced in BCC

To assess whether the methylation status impacts expression of the differential methylated genes, we performed quantitative real-time PCR on primary BCC $(n=6)$ and normal skin samples $(n=6)$. Whereas we could not detect SHH and RASSF1A mRNA in BCC and normal skin samples (data not shown), we did observe that SFRP5 mRNA expression level was significantly reduced in $B C C$ versus normal skin $(P=0.019)$. $A P C$ mRNA expression level was not altered $(P=0.937)$ in the samples examined (Figure 2A). Nevertheless, BCC with methylated APC showed borderline significant reduction mRNA level compared with $B C C$ in which $A P C$ was unmethylated $(P=0.050)$. Correlation of mRNA expression and methylation was not observed for SFRP5 ( $P=0.355)$ (Figure 2B), probably due to the fact that SFRP5 expression levels in all BCC were very low.

To investigate if we could see an effect of DNA methylation on the protein level, we performed immunohistochemical protein staining for SHH, APC and RASSF1A in a random selection of nodular BCC for which the methylation status had also been determined. After extensive testing, we found that commercially available antibodies for SFRP5 were not suitable for immunohistochemistry. Using the hair follicle as internal control (set at 100\% positivity), the expression of SHH, APC and RASSF1A in tumor cells was significantly lower (all p-values < 0.001) (Figure 2A). The intra-class correlation coefficients (ICC) for the scores of all stainings were high, with 0.87 for SHH, 0.8 for APC and 0.83 for RASSF1A. However, expression levels of methylated samples were not further reduced when compared with unmethylated samples (Figure 2C). Lastly, for SFRP5 we considered the immunohistochemical data available at the ProteinAtlas website by way of independent comparison with our data. For SFRP5, ProteinAtlas has data for one antibody (HPA019840) in six BCC and six squamous cell carcinomas, showing strong cytoplasmic and membranous antibody staining and with strong intensity in $>75 \%$ of the tumor cells as well as the overlying skin, which is in contrast with the low mRNA expression levels we found. We think that the ProteinAtlas samples are affected by overstaining. As a result, it is not possible to conclude from these data whether or not SFRP5 expression in BCC is lower than in unaffected skin, as it is in our series. Furthermore, heterogeneity among immunohistochemical stainings is a frequent problem interfering with any assessment and can be due to several factors including the antibody of choice. To settle this matter, more comprehensive SFRP5 expression analyses in a larger series of BCC, using both immunohistochemistry and quantitative RT-PCR, would be desirable. 
A
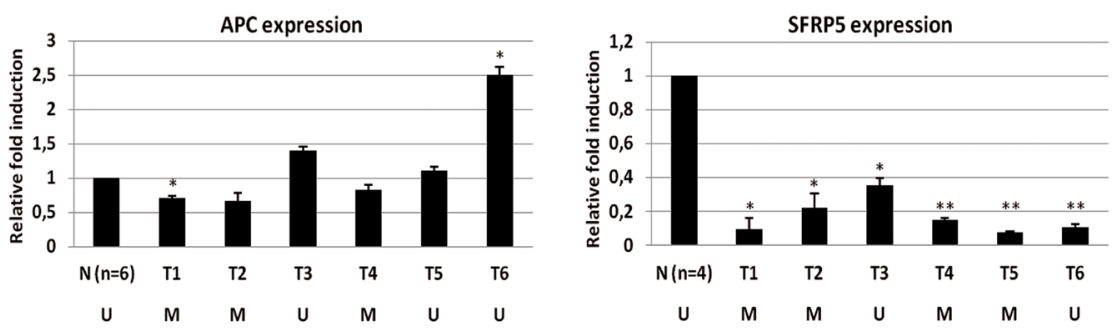

B
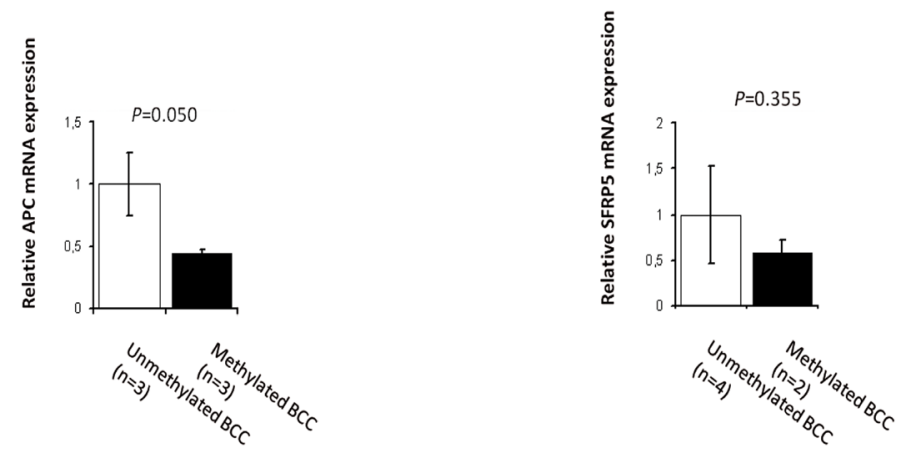

C
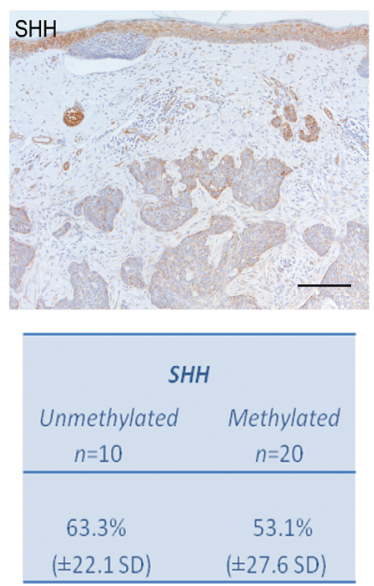

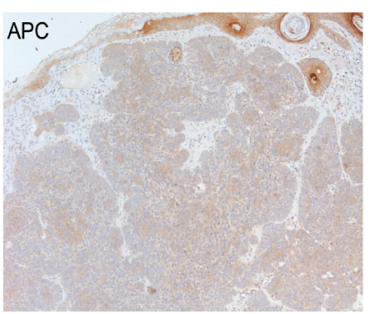

APC

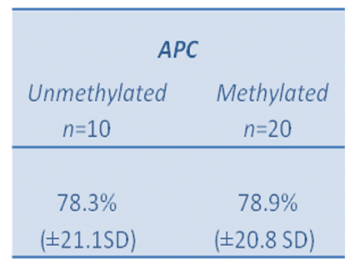

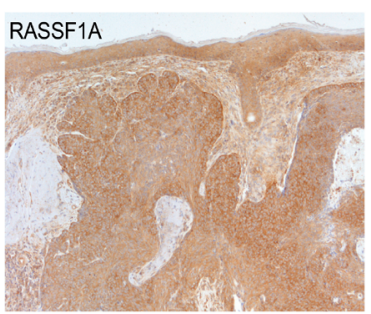

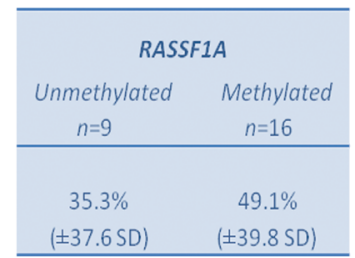

Figure 2. Expression of SHH, APC, SFRP5 and RASSF1A is reduced in BCC versus healthy skin control tissue.

A. Relative expression levels of APC and SFRP5 in BCC tissues as compared to the expression level in normal skin $(n=6)(2-\Delta \Delta C t)$. All reactions were done in triplicates, standard error of the mean (SEM) is shown as error bars. Expression levels were normalized to Cyclophilin A. U, unmethylated sample; M, methylated sample. ${ }^{*} p \leq 0.05,{ }^{* *} p \leq 0.001$. B. Relative mRNA expression in unmethylated versus methylated BCC samples for either APC or SFRP5. C. Microphotographs of selected samples of SHH, APC and RASSF1A. Bar $=200 \mu \mathrm{m}$. 
Low levels of nuclear B-catenin coincide with high levels of E-cadherin in BCC

As our results suggest epigenetic involvement of the WNT pathway in BCC, we subsequently analyzed the expression of $\beta$-catenin as readout for WNT pathway activity. $\beta$-catenin expression was localized at cell membranes of normal epidermis and within the cytoplasm and nuclei of hair follicles, consistent with the known activity of canonical WNT signaling in this structure..$^{18}$ In the 101 BCC we examined, $\beta$-catenin was present only at cell membranes in $65(64.4 \%)$ tumors, and localized at both the cell membrane and in the cytoplasm in 8 $(7.9 \%)$ cases. Nuclear staining was predominantly located at the tumor periphery and only seen in $28(27.7 \%)$ of the BCC samples without preference for one of the subtypes (Figure $3 A$ ). No correlation could be detected between the methylation status of APC or SFRP5 and the presence or localization of $\beta$-catenin. As it is known that the $E$-cadherin/ $\beta$-catenin complex is important in both cell adhesion and canonical WNT signaling ${ }^{46}$, we additionally analyzed the expression of E-cadherin, which is normally expressed throughout all layers of the epidermis, including hair follicles. ${ }^{47}$ Expression of E-cadherin in $59 \mathrm{BCC}$ was significantly lower compared with the normal epidermis $(P=0.001)$, but was rated high $(94.1 \%)$ in the tumor cells. Likewise, intensity of staining was rated as strong (69.5\%) in the BCC (Figure 3B). These findings were independent of tumor subtype and in agreement with the generally non-metastatic behavior of BCC. ${ }^{3}$ The ICC for E-cadherin (0.91) again was high.

A
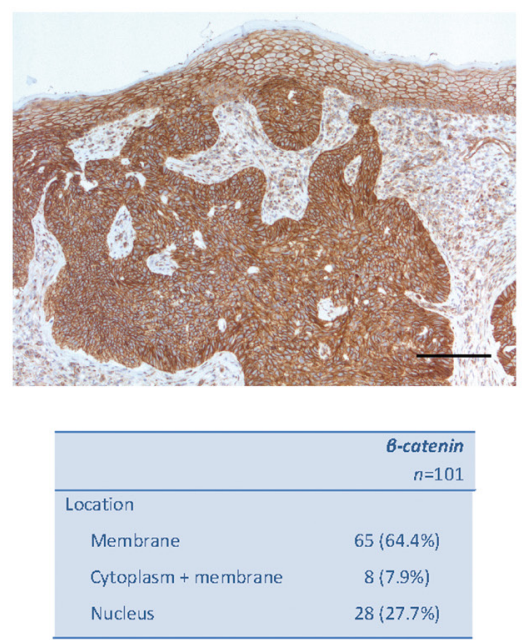

B

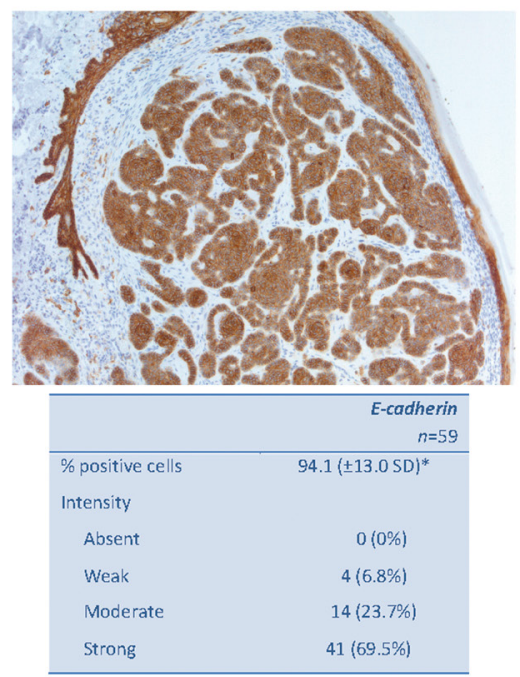

Figure 3. Low levels of nuclear B-catenin coincide with high levels of E-cadherin in BCC

A. Microphotographs of selected sample of B-catenin, showing nuclear staining only at the periphery or the tumor Bar $=200 \mu \mathrm{m}$. B. Microphotographs of selected sample of E-cadherin showing lowered expression of the tumor compared with the normal epidermis. 


\section{Discussion}

To the best of our knowledge, this is the largest study to date addressing epigenetic changes in the most common human malignancy, BCC. We provide data which suggest that the $\mathrm{SHH}$ and WNT pathways may be epigenetically involved in BCC pathobiology, since both networks are affected by aberrant promoter methylation of SHH, APC and SFRP5. Aberrant methylation and associated gene silencing of APC and SFRP5 may contribute to the pathogenesis of BCC by impairing negative regulation of WNT pathway activity. In addition, we detected differential methylation of RASSF1A, a well-known tumor suppressor modulating a broad range of cellular functions that are essential for normal cell growth. RASSF1A expression is lost in high frequency by promoter methylation in a wide variety of human tumors, in fact, it is one of the best-characterized tumor suppressor genes in UV-induced squamous cell carcinoma of the skin $^{48,49}$ and methylation of its promoter had been previously demonstrated in BCC. ${ }^{28}$

Epigenetic alterations in BCC have previously been reported, but in limited sample series 26-30 and without providing further experimental evidence for relevance of the epigenetic changes for BCC carcinogenesis. Sathyanarayana et.al. investigated promoter methylation of 12 genes in a series of 68 BCC samples and 58 non-malignant lesions (skin tags) and showed that laminin gamma 2 ( $L A M C 2)$, cadherin $1(C D H 1)$ and RASSF1A were significantly more often methylated in BCC. These findings are in agreement with our results on RASSF1A methylation, even though we found higher methylation frequencies in both BCC and normal skin. A possible explanation for this is that we performed nested MSP, which is a very sensitive technique for methylation analysis on FFPE tissue, while Sathyanarayana et al. performed direct MSP, which is less sensitive. In addition, they investigated the effect of sun exposure on promoter methylation in skin tags and cancer, and found similar methylation frequencies for the genes examined. From this, it was concluded that promoter hypermethylation in general was more likely related to sun exposure rather than being tumor-specific. In our study, we could not detect comparable methylation patterns in sun-exposed skin and BCC for SHH APC, SFRP5 and RASSF1A. Moreover, normal SE and SP skin samples showed similar methylation patterns, suggesting that the methylation we observed is tumor-specific. It is of interest that methylation patterns in sun-exposed skin did not resemble those in BCC, as sun exposure is the main risk factor in the development of BCC. ${ }^{50}$ Thus, our findings do not support a contribution of UVB exposure to the observed promoter hypermethylation of $\mathrm{SHH}$, APC, SFRP5 and RASSF1A. The intriguing observation that $\mathrm{nBCC}$ and $\mathrm{BCC}$ harbored more frequent methylation for these four genes compared with SBCC might indicate that DNA methylation contributes to the development or evolution of BCC. It is tempting to speculate that increased silencing of putative tumor suppressors correlates with increasingly invasive behavior, a notion that is supported by our finding of differential SFRP5 methylation in iBCC versus $\mathrm{nBCC}$ and $\mathrm{sBCC}$. The increased $\mathrm{SHH}$ methylation is probably an epiphenomenon, as $\mathrm{SHH}$ is no longer required for $\mathrm{SMOH}$ activity in the majority of BCC. As an alternative explanation, $\mathrm{SHH}$ silencing might contribute through as yet unidentified pathways to tumorigenesis. 
To confirm functionally relevant methylation, i.e., transcriptional silencing of affected genes, we performed gene expression analyses with RT-PCR on FF and immunohistochemical staining on FFPE samples with verified methylation status. Our mRNA expression analysis of APC and SFRP5 in patient and control samples confirmed a down regulation of these genes in samples harboring methylation. By immunohistochemistry, expression of SHH, APC and RASSF1A (all p-values < 0.001) was lowered in BCC as compared to normal skin. We did not detect a direct correlation between methylation status and expression levels as visualized by immunohistochemistry. We think that this observation can be explained by the two-way detection method in immunohistochemical staining intensifying the original signal. Also, mRNA levels do not always correlate directly with protein levels. ${ }^{51}$

These data together support our hypothesis of epigenetic involvement of the SHH and WNT pathways in BCC pathogenesis. Promoter hypermethylation-mediated silencing of negative regulators of WNT signaling is consistent with activity of this pathway, however available literature concerning activity of the WNT pathway in BCC is ambiguous. Therefore we assessed activation of canonical WNT signaling by determining $\beta$-catenin intracellular localization. $\beta$-catenin, a key WNT effector, is a membrane-bound protein which accumulates in the cytoplasm and subsequently translocates to the nucleus when activated. ${ }^{52}$ In BCC, the literature concerning immunohistochemical analysis of $\beta$-catenin is ambiguous. ${ }^{19,53-55}$ El-Bahrawy et al. ${ }^{53}$ showed nuclear staining in $55 \%(n=56)$ of $B C C$, mostly at the periphery of the tumors, while only $23 \%$ of the 86 BCC examined by Saldanha et al. ${ }^{54}$ had $\beta$-catenin positive nuclei. No correlation between $\beta$-catenin localization and histological subtype was seen in either study. Furthermore, in a paper recently published by Pourreyron et al. ${ }^{19}$, nuclear $\beta$-catenin was absent in all examined BCC $(n=7) .{ }^{19}$ In our series, we demonstrated nuclear $\beta$-catenin in 28 of the $101(27.7 \%)$ BCC examined, which is consistent with previous reports. It would be of interest in this context to examine Axin 2 expression levels in BCC as an additional marker for canonical WNT activity. ${ }^{19}$ We reasoned that the lack of $\beta$-catenin nuclear localization might be due to E-cadherin co-expression, as high levels of E-cadherin can prevent nuclear translocation of $\beta$-catenin. ${ }^{46,56}$ Indeed our data show high levels of E-cadherin expression in BCC, with $94.1 \%$ of the cells being positive, although significantly lowered when compared to adjacent normal epidermis. Thus, the absence of nuclear $\beta$-catenin in many cases may be due to high E-cadherin levels, which would also be consistent with the general inability of BCC to metastasize. ${ }^{50}$

It is of considerable interest that the epigenetic changes we found parallel the genetic changes driving BCC growth, in particular the silencing of known WNT pathway inhibitors. These observations support a biological relevance of gene silencing by promoter hypermethylation in BCC. Moreover, they are consistent with a model in which epigenetic changes help to drive BCC tumor growth through deregulation of the WNT pathway, upon initiation of growth by mutations affecting $\mathrm{SHH}$ signaling. Previous work has demonstrated that $\mathrm{BCC}$ growth requires WNT signaling. ${ }^{18,19}$ WNT pathway effectors are among the target genes of $\mathrm{SHH}$, providing a rationale for this positive feedback mechanism. Our findings uncover a new level of regulation; inactivation of WNT inhibitors may be equally important as increased activity of WNT effectors. 
In conclusion, we provide evidence that promoter hypermethylation-mediated silencing of known and putative TSGs is present in BCC. The extent to which these epigenetic changes actively contribute to BCC development will be more fully charted in order to determine whether DNA demethylation could be a viable strategy for BCC treatment. 


\section{References}

1. Szeimies RM, Karrer S. Towards a more specific therapy: targeting nonmelanoma skin cancer cells. The British journal of dermatology 2006;154 Suppl 1:16-21.

2. Holterhues C, Vries E, Louwman MW, Koljenovic S, Nijsten T. Incidence and trends of cutaneous malignancies in the Netherlands, 1989-2005. The Journal of investigative dermatology 2010;130:1807-12.

3. Roewert-Huber J, Lange-Asschenfeldt B, Stockfleth E, Kerl H. Epidemiology and aetiology of basal cell carcinoma. The British journal of dermatology 2007;157 Suppl 2:47-51.

4. von Domarus H, Stevens PJ. Metastatic basal cell carcinoma. Report of five cases and review of 170 cases in the literature. Journal of the American Academy of Dermatology 1984;10:1043-60.

5. Tilli CM, Van Steensel MA, Krekels GA, Neumann HA, Ramaekers FC. Molecular aetiology and pathogenesis of basal cell carcinoma. The British journal of dermatology 2005;152:1108-24.

6. Mosterd K, Arits AH, Thissen MR, Kelleners-Smeets NW. Histology-based treatment of basal cell carcinoma. Acta dermato-venereologica 2009;89:454-8.

7. Smeets NW, Krekels GA, Ostertag JU, et al. Surgical excision vs. Mohs' micrographic surgery for basal-cell carcinoma of the face: randomised controlled trial. Lancet 2004;364:1766-72.

8. Bonifas JM, Bare JW, Kerschmann RL, Master SP, Epstein EH, Jr. Parental origin of chromosome 9q22.3-q31 lost in basal cell carcinomas from basal cell nevus syndrome patients. Human molecular genetics 1994;3:447-8.

9. Gailani MR, Stahle-Backdahl M, Leffell DJ, et al. The role of the human homologue of Drosophila patched in sporadic basal cell carcinomas. Nature genetics 1996;14:78-81.

10. Hahn H, Wojnowski L, Miller G, Zimmer A. The patched signaling pathway in tumorigenesis and development: lessons from animal models. Journal of molecular medicine (Berlin, Germany) 1999;77:459-68.

11. Reifenberger J, Wolter M, Knobbe CB, et al. Somatic mutations in the PTCH, SMOH, SUFUH and TP53 genes in sporadic basal cell carcinomas. The British journal of dermatology 2005;152:43-51.

12. Epstein EH. Basal cell carcinomas: attack of the hedgehog. Nature reviews Cancer 2008;8:743-54.

13. Reifenberger J, Wolter M, Weber RG, et al. Missense mutations in $\mathrm{SMOH}$ in sporadic basal cell carcinomas of the skin and primitive neuroectodermal tumors of the central nervous system. Cancer research 1998;58:1798-803.

14. Xie J, Murone M, Luoh SM, et al. Activating Smoothened mutations in sporadic basal-cell carcinoma. Nature 1998;391:90-2.

15. Zedan W, Robinson PA, Markham AF, High AS. Expression of the Sonic Hedgehog receptor "PATCHED" in basal cell carcinomas and odontogenic keratocysts. The Journal of pathology 2001;194:473-7.

16. Donovan J. Review of the hair follicle origin hypothesis for basal cell carcinoma. Dermatol Surg 2009;35:1311-23.

17. Dahmane N, Lee J, Robins P, Heller P, Ruiz i Altaba A. Activation of the transcription factor Gli1 and the Sonic hedgehog signalling pathway in skin tumours. Nature 1997;389:876-81.

18. Yang SH, Andl T, Grachtchouk V, et al. Pathological responses to oncogenic Hedgehog signaling in skin are dependent on canonical Wnt/beta3-catenin signaling. Nature genetics 2008;40:1130-5.

19. Pourreyron C, Reilly L, Proby C, et al. Wnt5a is strongly expressed at the leading edge in non-melanoma skin cancer, forming active gradients, while canonical Wnt signalling is repressed. PloS one 2012; $7:$ e31827.

20. Herman JG, Baylin SB. Gene silencing in cancer in association with promoter hypermethylation. The New England journal of medicine 2003;349:2042-54.

21. Schuebel KE, Chen W, Cope L, et al. Comparing the DNA hypermethylome with gene mutations in human colorectal cancer. PLoS genetics 2007;3:1709-23. 
22. Giacinti L, Vici P, Lopez M. Epigenome: a new target in cancer therapy. La Clinica terapeutica 2008; 159:347-60.

23. Hatziapostolou M, Iliopoulos D. Epigenetic aberrations during oncogenesis. Cellular and molecular life sciences : CMLS 2011;68:1681-702.

24. Issa JP, Kantarjian HM. Targeting DNA methylation. Clinical cancer research : an official journal of the American Association for Cancer Research 2009;15:3938-46.

25. Abdulla FR, Feldman SR, Williford PM, Krowchuk D, Kaur M. Tanning and skin cancer. Pediatric dermatology 2005;22:501-12.

26. Goldberg M, Rummelt C, Laerm A, Helmbold P, Holbach LM, Ballhausen WG. Epigenetic silencing contributes to frequent loss of the fragile histidine triad tumour suppressor in basal cell carcinomas. The British journal of dermatology 2006;155:1154-8.

27. Heitzer E, Bambach I, Dandachi N, Horn M, Wolf P. PTCH promoter methylation at low level in sporadic basal cell carcinoma analysed by three different approaches. Experimental dermatology 2010;19:926-8.

28. Sathyanarayana UG, Moore AY, Li L, et al. Sun exposure related methylation in malignant and non-malignant skin lesions. Cancer letters 2007;245:112-20.

29. van Doorn R, Gruis NA, Willemze R, van der Velden PA, Tensen CP. Aberrant DNA methylation in cutaneous malignancies. Seminars in oncology 2005;32:479-87.

30. Wu W, Zhang J, Yang H, Shao Y, Yu B. Examination of AKAP12 promoter methylation in skin cancer using methylation-sensitive high-resolution melting analysis. Clinical and experimental dermatology 2011;36:3815 .

31. Comprehensive molecular characterization of human colon and rectal cancer. Nature 2012;487:330-7.

32. Lee DA, Grossman ME, Schneiderman P, Celebi JT. Genetics of skin appendage neoplasms and related syndromes. Journal of medical genetics 2005;42:811-9.

33. Borkowska J, Schwartz RA, Kotulska K, Jozwiak S. Tuberous sclerosis complex: tumors and tumorigenesis. International journal of dermatology 2011;50:13-20.

34. van Steensel MA, van Geel M, Badeloe S, Poblete-Gutierrez P, Frank J. Molecular pathways involved in hair follicle tumor formation: all about mammalian target of rapamycin? Experimental dermatology 2009;18:18591.

35. Richter AM, Pfeifer GP, Dammann RH. The RASSF proteins in cancer; from epigenetic silencing to functional characterization. Biochimica et biophysica acta 2009;1796:114-28.

36. Derks S, Lentjes MH, Hellebrekers DM, de Bruine AP, Herman JG, van Engeland M. Methylation-specific PCR unraveled. Cell Oncol 2004;26:291-9.

37. Herman JG, Graff JR, Myohanen S, Nelkin BD, Baylin SB. Methylation-specific PCR: a novel PCR assay for methylation status of $\mathrm{CpG}$ islands. Proceedings of the National Academy of Sciences of the United States of America 1996;93:9821-6.

38. van Engeland M, Roemen GM, Brink M, et al. K-ras mutations and RASSF1A promoter methylation in colorectal cancer. Oncogene 2002;21:3792-5.

39. Livak KJ, Schmittgen TD. Analysis of relative gene expression data using real-time quantitative PCR and the 2(-Delta Delta C(T)) Method. Methods 2001;25:402-8.

40. Kundel HL, Polansky M. Measurement of observer agreement. Radiology 2003;228:303-8.

41. Shrout PE, Fleiss JL. Intraclass correlations: uses in assessing rater reliability. Psychological bulletin 1979;86:420-8.

42. Rosner B. Fundamentals of biostatistics. . 6 ed. Belmont: Duxbury.2006.

43. Veeck J, Geisler C, Noetzel E, et al. Epigenetic inactivation of the secreted frizzled-related protein-5 (SFRP5) gene in human breast cancer is associated with unfavorable prognosis. Carcinogenesis 2008;29:991-8. 
44. House MG, Guo M, lacobuzio-Donahue C, Herman JG. Molecular progression of promoter methylation in intraductal papillary mucinous neoplasms (IPMN) of the pancreas. Carcinogenesis 2003;24:193-8.

45. Chhibber A, Schroeder BG. Single-molecule polymerase chain reaction reduces bias: application to DNA methylation analysis by bisulfite sequencing. Analytical biochemistry 2008;377:46-54.

46. Tian X, Liu Z, Niu B, et al. E-cadherin/beta-catenin complex and the epithelial barrier. Journal of biomedicine \& biotechnology 2011;2011:567305.

47. Bezdekova M, Brychtova S, Sedlakova E, et al. Immunohistochemical assessment of E-cadherin and beta-catenin in trichofolliculomas and trichoepitheliomas. Biomedical papers of the Medical Faculty of the University Palacky, Olomouc, Czechoslovakia 2007;151:251-5.

48. Donninger H, Vos MD, Clark GJ. The RASSF1A tumor suppressor. Journal of cell science 2007;120:316372.

49. Nandakumar V, Vaid M, Tollefsbol TO, Katiyar SK. Aberrant DNA hypermethylation patterns lead to transcriptional silencing of tumor suppressor genes in UVB-exposed skin and UVB-induced skin tumors of mice. Carcinogenesis 2011;32:597-604.

50. Madan V, Lear JT, Szeimies RM. Non-melanoma skin cancer. Lancet 2010;375:673-85.

51. Gort EH, Suijkerbuijk KP, Roothaan SM, et al. Methylation of the TWIST1 promoter, TWIST1 mRNA levels, and immunohistochemical expression of TWIST1 in breast cancer. Cancer Epidemiol Biomarkers Prev 2008; 17:3325-30.

52. MacDonald BT, Tamai K, He X. Wnt/beta-catenin signaling: components, mechanisms, and diseases. Developmental cell 2009;17:9-26.

53. El-Bahrawy M, El-Masry N, Alison M, Poulsom R, Fallowfield M. Expression of beta-catenin in basal cell carcinoma. The British journal of dermatology 2003;148:964-70.

54. Saldanha G, Ghura V, Potter L, Fletcher A. Nuclear beta-catenin in basal cell carcinoma correlates with increased proliferation. The British journal of dermatology 2004;151:157-64.

55. Fukumaru K, Yoshii N, Kanzaki T, Kanekura T. Immunohistochemical comparison of beta-catenin expression by human normal epidermis and epidermal tumors. The Journal of dermatology 2007;34:746-53.

56. Li L, Zhang Z, Li B, Gao F, Jonas JB. E-cadherin and beta-catenin expression in sebaceous eyelid adenocarcinomas. Graefe's archive for clinical and experimental ophthalmology = Albrecht von Graefes Archiv fur klinische und experimentelle Ophthalmologie 2011;249:1867-73. 
Epigenetic changes in basal cell carcinoma affect SHH and WNT signaling components | 53
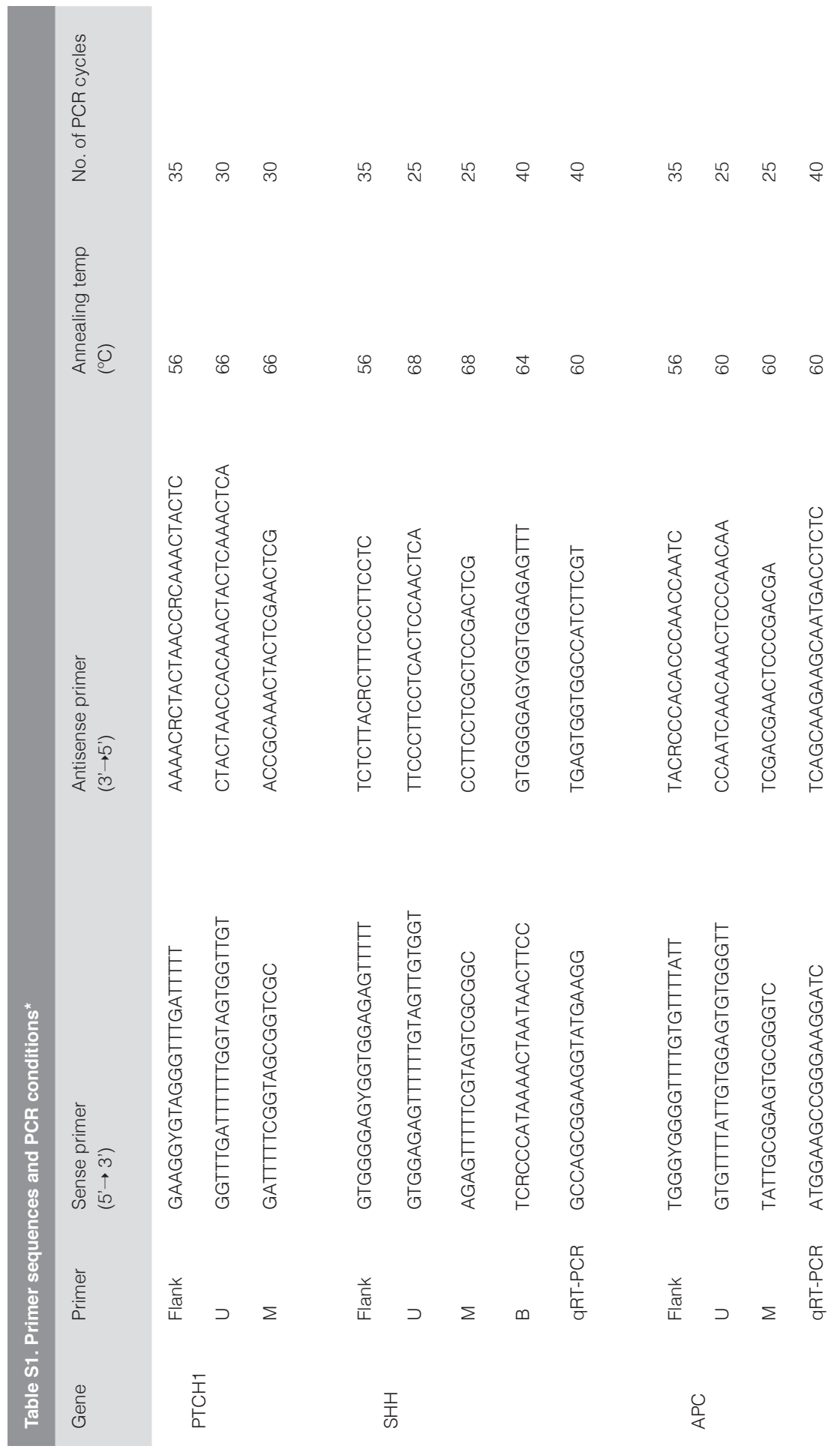


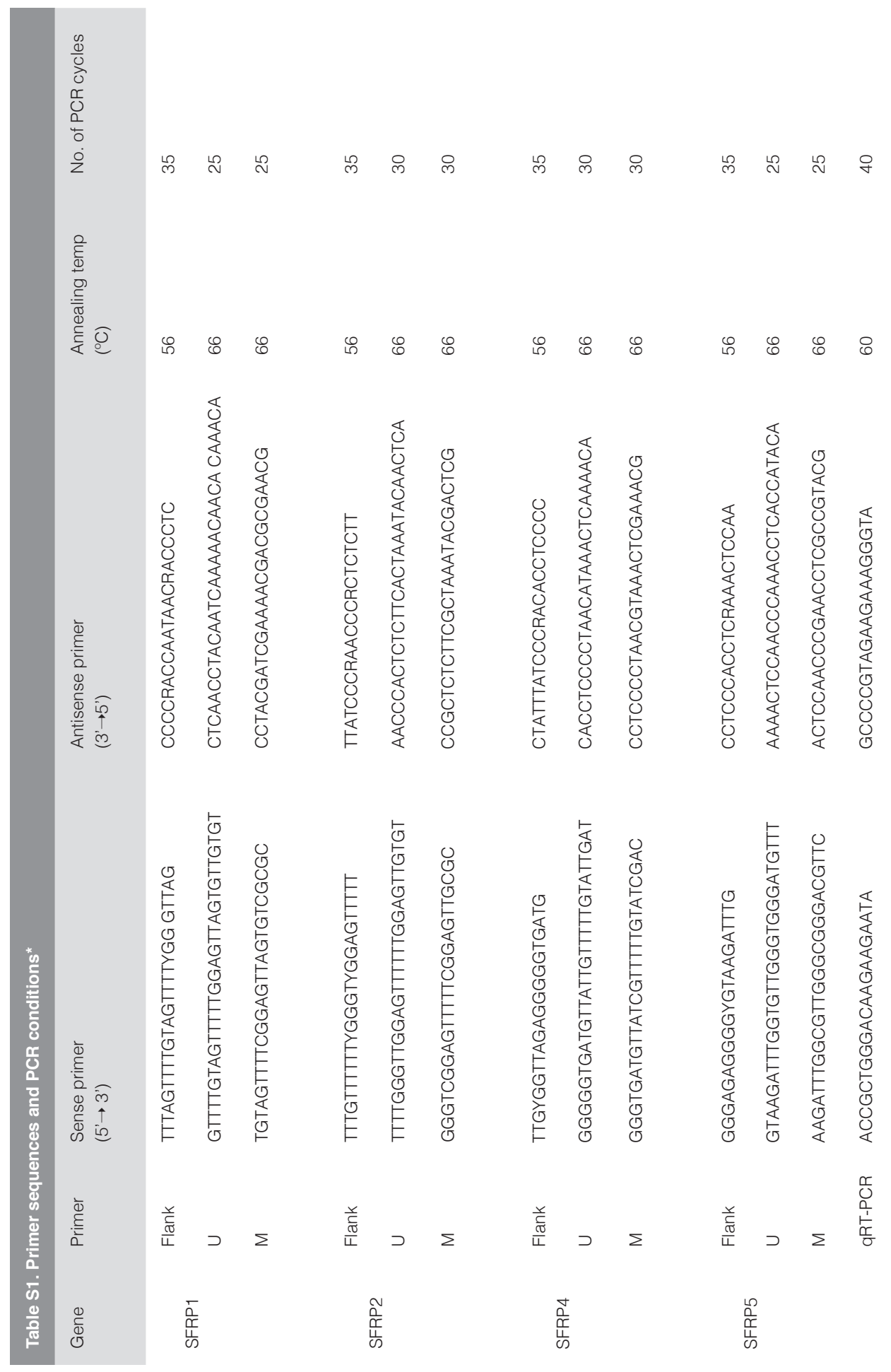


Epigenetic changes in basal cell carcinoma affect SHH and WNT signaling components | 55
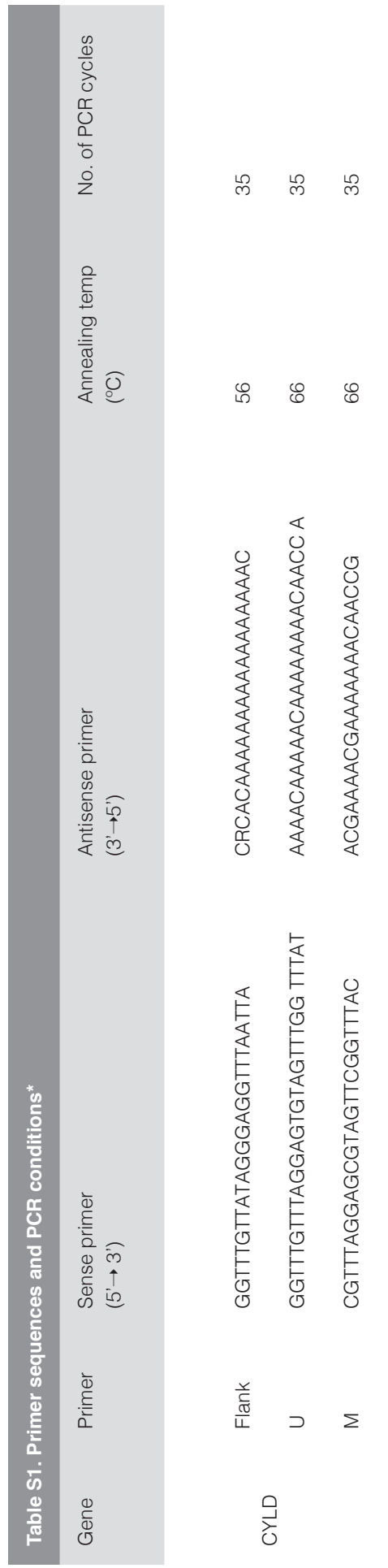

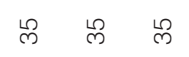

œ 户 \&

(1)

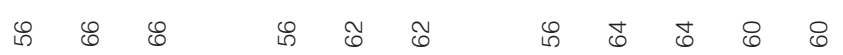

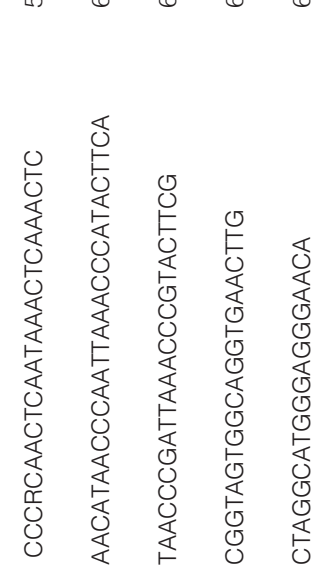

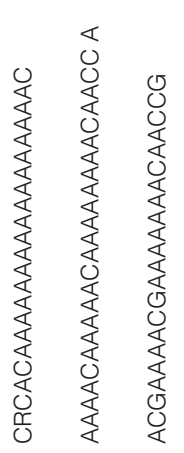

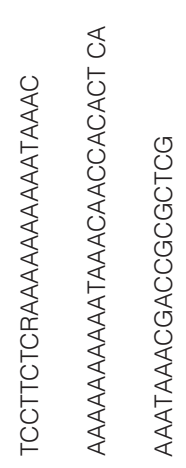
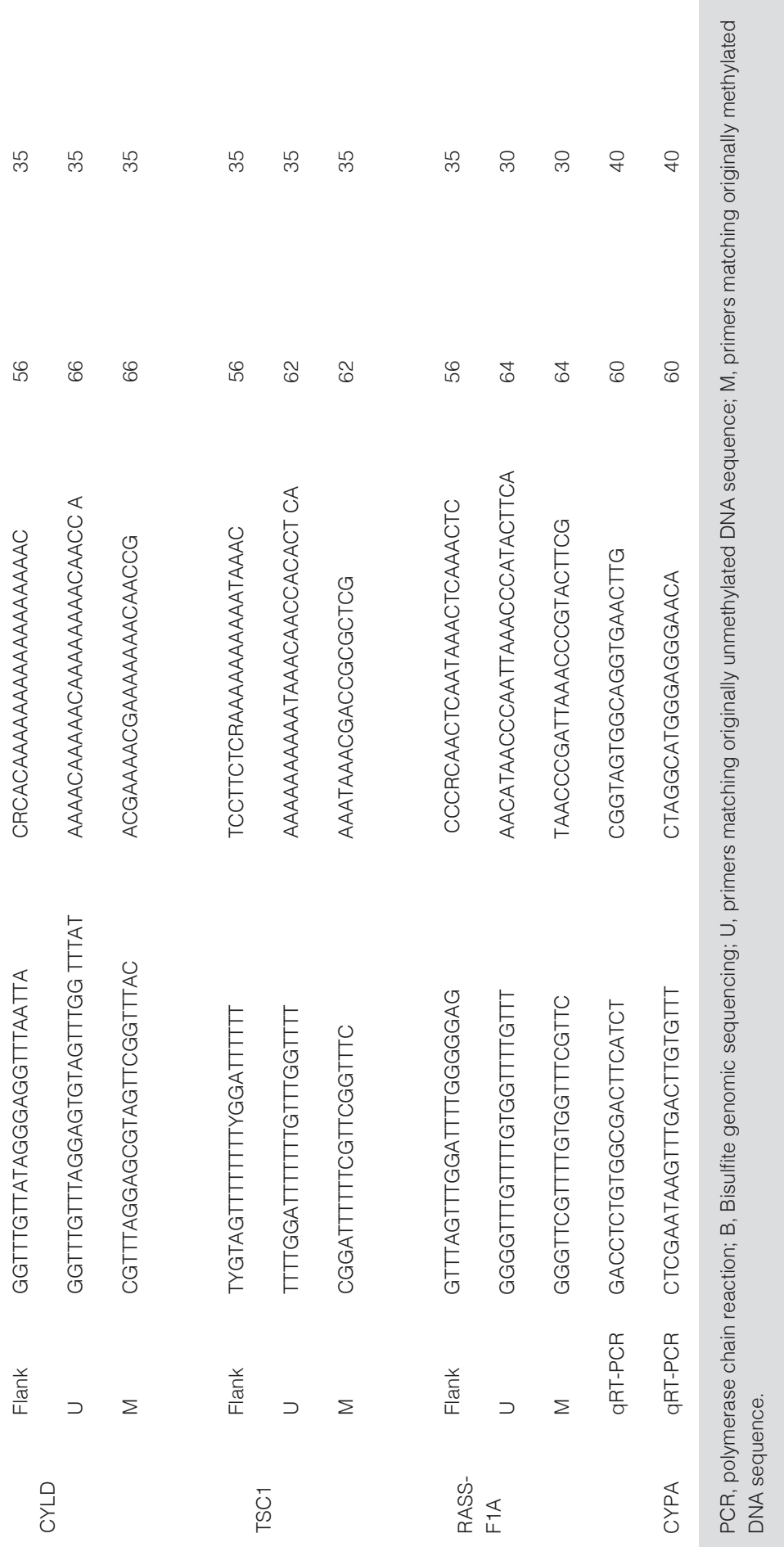


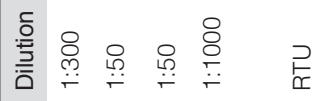

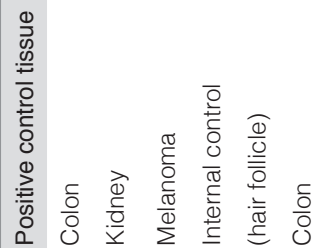

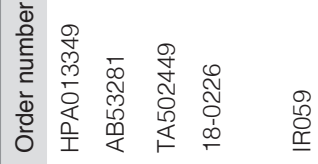

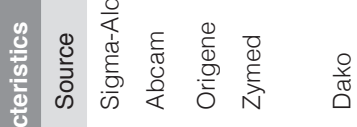

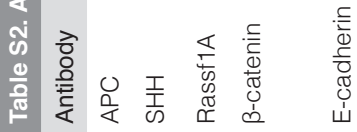






\section{Chapter 2.2}

\section{Functional epigenomics implicates}

inflammatory signaling in

\section{basal cell carcinoma pathogenesis}

Tjinta Brinkhuizen*, Simon L.I.J. Denil*, Tim De Meyer, Wim Van Criekinge, Klara Mosterd, Manon van Engeland, Maurice A.M van Steensel

*Both authors contributed equally to this paper and should be considered as first authors.

Submitted at the Journal of Investigative Dermatology 
Basal cell carcinoma (BCC) is the most common malignancy in Caucasians. ${ }^{1}$ To explore the impact of epigenetic regulation on BCC carcinogenesis, we integrated MethylCap-sequencing $^{2}$ on $5 \mathrm{BCC}$ and 5 healthy human skin samples with RNA-sequencing data from 3 $\mathrm{BCC}$ and 3 control samples. The combined data constitute the functional methylome. We correlated methylation with RNA expression and identified 15 differentially methylated or expressed loci in $\mathrm{BCC}$, among which PTCH $1{ }^{1}, \mathrm{PTPRN}^{3}$ and IFI30. ${ }^{4}$ In silico pathway analysis using the expression data firmly implicated cytokine signaling in BCC growth. Pathway activity was confirmed with immunohistochemical staining. We identified significant epigenetic modulation of gene expression in BCC and uncovered immune modulatory signaling as a driver in BCC development. We translated these findings into a trial with topical application of cyclooxygenase inhibitors to BCC, yielding excellent treatment responses.

Basal cell carcinoma (BCC) mostly affects people aged 60 years or older. Its incidence is rising by $10 \%$ each year worldwide, reaching epidemic proportions. ${ }^{1}$ The average lifetime risk for Caucasians to develop a BCC is up to $30 \% .{ }^{5}$ About $40-50 \%$ of patients presenting with a primary $\mathrm{BCC}$ will develop one or more further BCC within 5 years. ${ }^{1} \mathrm{BCC}$ rarely metastasizes, but can cause extensive local tissue destruction when left untreated. ${ }^{6}$ Surgical excision is the current standard of treatment, with cure rates of roughly $95 \%,{ }^{1,7}$ However, surgery is costly and time-consuming. The ever-increasing number of tumors will thus generate high costs and workload, putting a significant burden on health-care systems. Hence, BCC is becoming a serious public health problem and there is a clear need for more cost-effective and simple treatment strategies. In order to develop these, a thorough understanding of BCC biology is required.

It is well established that aberrant Sonic Hedgehog $(\mathrm{SHH})$ signaling due to mutations in one of its components is central to BCC carcinogenesis. ${ }^{8}$ However, a recent study of the BCC exome showed an extremely high mutational load, suggesting that BCC could be a heterogeneous malignancy, whose growth might be sustained by mechanisms other than $\mathrm{SHH}$ signaling. ${ }^{9}$

It is known that, in addition to genetic mutations, epigenetic alterations such as DNA methylation play an important role in carcinogenesis, occurring very frequently in a variety of tumor types. ${ }^{10}$ DNA methylation consists of adding a methyl moiety to cytosine nucleotides followed by a guanine (CpG dinucleotide). These CpG dinucleotides are clustered (CpG islands) and are usually not methylated when associated with the promoter region of the gene. Cancer-related hypermethylation of the promoter region results in silencing of crucial tumor suppressor genes, in addition to mutational changes. ${ }^{11}$ Based on results from a pilot study, we reasoned that methylation might contribute to BCC biology. ${ }^{12}$ To understand how, we combined as first genome-wide methylated DNA enrichment sequencing (MethylCap-seq) 2 and single-end RNA sequencing on primary BCC and healthy skin controls. We sequenced methylated $\mathrm{CpG}(\mathrm{mCpG})$ enriched DNA of five $\mathrm{BCC}$ and five control samples of healthy skin from unaffected persons to obtain an overview of the BCC methylome (see Supplementary Table S1 for patients characteristics and Supplementary methods 1.1). In a first approach, we examined 32979 methylation regions in 22121 genes selected for their potential information content (see Methods). Of these regions, 1 gene was significantly dif- 
ferentially methylated between BCC and unaffected skin (False Discovery Rate (FDR) < 0.05) and 12 with FDR $<0.1$, see Table 1 , see Supplementary Table S2 for full list). Noteworthy is CDKN2A, which codes for two tumor suppressor genes (p14 and p16) and is involved in the negative control of cell proliferation in BCC. ${ }^{13}$ IFI30 is of additional interest, playing a critical immunoregulatory role in melanoma by mediating antitumor immunity via CD4+ T-cells. ${ }^{4}$

\begin{tabular}{llrrrr}
\multicolumn{5}{l}{ Table 1. Significantly differentially methylated loci in bcc tissue versus healthy control tissue. } \\
Ensembl.Gene.ld & Gene Label & Chr & Begin & End & FDR \\
\hline ENSG00000104879 & CKM & 19 & 45826253 & 45826629 & 0,0496 \\
ENSG00000147889 & CDKN2A & 9 & 21970816 & 21971287 & 0,0596 \\
ENSG00000095066 & HOOK2 & 19 & 12876661 & 12877365 & 0,0657 \\
ENSG00000078399 & HOXA9 & 7 & 27204556 & 27205337 & 0,0704 \\
ENSG00000111452 & GPR133 & 12 & 131513956 & 131514262 & 0,0773 \\
ENSG00000187909 & AC078925.1 & 12 & 131513956 & 131514262 & 0,0773 \\
ENSG00000183876 & ARSI & 5 & 149681603 & 149681936 & 0,0822 \\
ENSG00000100418 & PPPDE2 & 22 & 41995921 & 41996177 & 0,0858 \\
ENSG00000172346 & CSDC2 & 22 & 41995921 & 41996177 & 0,0858 \\
ENSG00000196155 & PLEKHG4 & 16 & 67322714 & 67322881 & 0,0889 \\
ENSG00000186891 & TNFRSF18 & 1 & 1141672 & 1142148 & 0,0921 \\
ENSG00000165588 & OTX2 & 14 & 57270914 & 57271235 & 0,0952 \\
ENSG00000216490 & IFl30 & 19 & 18282492 & 18282589 & 0,0980 \\
\hline Chr: chromosome, FDR: False Discovery Rate & & & &
\end{tabular}

As methylation can influence gene expression levels ${ }^{14}$, we decided to correlate the methylation results to global transcriptome data, aiming to determine the 'functional methylome' (see Supplementary Methods 1.2). To obtain genome-wide expression data, we performed rRNA depleted, strand specific, total RNA sequencing (RNA-seq henceforth) on three independent primary BCC samples and three independent, age- and sex-matched, healthy glabrous skin samples. We ranked 51984 Ensembl gene identifiers with detectable expression levels according to differential expression between tumors and controls (see Supplementary Table S3). Fifty-eight differentially expressed genes were identified using FDR $<0.1$. The top twenty differentially expressed genes at FDR $<0.05$ are listed in Table 2 and include genes affecting inter- and intracellular structure (such as MMP13 ${ }^{15}$, MMP9 $\left.^{16}, \mathrm{ACTN2}^{17}, \mathrm{CDHR} 1\right)$, genes that facilitate apoptotic signaling (GMZK ${ }^{18}, \mathrm{GZMB}^{19}$ ), tumor suppressor genes $\left(\mathrm{DCC}^{20}\right)$ and immune signaling genes (XCL2 $\left.{ }^{21}, \mathrm{CXCL} 10^{22}\right)$. 


\begin{tabular}{|c|c|c|c|c|c|}
\hline Ensembl.Gene.Id & Gene Label & Chr & Begin & End & FDR \\
\hline ENSG00000137745 & MMP13 & 11 & 102813724 & 102826463 & 0,0199 \\
\hline ENSG00000212195 & U3 & 17 & 56709003 & 56709197 & 0,0255 \\
\hline ENSG00000244734 & $\mathrm{HBB}$ & 11 & 5246694 & 5250625 & 0,0274 \\
\hline ENSG00000202077 & RNU1-60P & 17 & 56736510 & 56736666 & 0,0290 \\
\hline ENSG00000143185 & CXCL2 & 1 & 168510003 & 168513235 & 0,0304 \\
\hline ENSG00000187323 & DCC & 18 & 49866542 & 51057784 & 0,0313 \\
\hline ENSG00000118785 & SPP1 & 4 & 88896819 & 88904562 & 0,0323 \\
\hline ENSG00000187689 & AMTN & 4 & 71384257 & 71398459 & 0,0344 \\
\hline ENSG00000232775 & AP000525.10 & 22 & 16162066 & 16172700 & 0,0356 \\
\hline ENSG00000211526 & AL121869.1 & $x$ & 91715451 & 91715544 & 0,0366 \\
\hline ENSG00000100453 & GZMB & 14 & 25100160 & 25103473 & 0,0382 \\
\hline ENSG00000077522 & ACTN2 & 1 & 236849754 & 236927931 & 0,0395 \\
\hline ENSG00000238274 & CASC15 & 6 & 22056775 & 22111157 & 0,0409 \\
\hline ENSG00000148600 & CDHR1 & 10 & 85954410 & 85979377 & 0,0422 \\
\hline ENSG00000100985 & MMP9 & 20 & 44637547 & 44645200 & 0,0433 \\
\hline ENSG00000113088 & GZMK & 5 & 54320081 & 54330398 & 0,0445 \\
\hline ENSG00000125869 & LAMP5 & 20 & 9495008 & 9511171 & 0,0456 \\
\hline ENSG00000131203 & IDO5 & 8 & 39759794 & 39785963 & 0,0469 \\
\hline ENSG00000160321 & ZNF208 & 19 & 22148897 & 22193745 & 0,0484 \\
\hline ENSG00000169245 & CXCL10 & 4 & 76942273 & 76944650 & 0,0498 \\
\hline
\end{tabular}

Based on the RNA-seq data, we repeated the differential methylation analysis in 15983 selected methylation regions located in 643 genes that are likely differentially expressed (see Methods). Fifteen of these regions were significantly differentially methylated (FDR $<0.1$, listed in Table 3 Supplemental Table S4). Notable hits include PTCH1 (part of SHH pathway), PTPRN2 (methylation marker in squamous lung cell carcinoma3) and IFI30.4 It should be noted that there was little overlap between the top-ranked differentially methylated loci and differentially expressed genes $(F D R<0.1)$ even when preselecting for genes which might be differentially expressed in BCC. 


\begin{tabular}{llcccc}
\multicolumn{5}{l}{ Table 3. Significantly differentially methylated 'functional' loci in bcc tissue versus healthy control tissue. } \\
\hline Ensembl.Gene.Id & Gene Label & Chr & Begin & End & FDR \\
\hline ENSG00000104879 & CKM & 19 & 45826253 & 45826629 & 0,0246 \\
ENSG00000185920 & PTCH1 & 9 & 98218565 & 98218860 & 0,0383 \\
ENSG00000186439 & TRDN & 6 & 123680870 & 123681186 & 0,0481 \\
ENSG00000155093 & PTPRN2 & 7 & 157916451 & 157916924 & 0,0535 \\
ENSG00000155093 & PTPRN2 & 7 & 158135892 & 158136224 & 0,0572 \\
ENSG00000107518 & ATRNL1 & 10 & 117676548 & 117676940 & 0,0602 \\
ENSG00000231200 & AC068490.2 & 2 & 22404105 & 22404340 & 0,0623 \\
ENSG00000216490 & IFI30 & 19 & 18282492 & 18282589 & 0,0655 \\
ENSG00000182870 & GALNT9 & 12 & 132896405 & 132896746 & 0,0714 \\
ENSG00000155093 & PTPRN2 & 7 & 157486385 & 157486857 & 0,0756 \\
ENSG00000105963 & ADAP1 & 7 & 949072 & 949345 & 0,0815 \\
ENSG00000240230 & COX19 & 7 & 9449072 & 949345 & 0,0815 \\
ENSG00000155093 & PTPRN2 & 7 & 157477818 & 157478450 & 0,0866 \\
ENSG00000077522 & ACTN2 & 1 & 236860004 & 236860257 & 0,0914 \\
ENSG00000164176 & EDIL3 & 5 & 83598091 & 83598405 & 0,0995 \\
ENSG00000186732 & MPPED1 & 43822136 & & 0,0998 \\
\hline Chr: chrom050me, FDR: False Discovery Rate & & & 43822777 & \\
\hline
\end{tabular}

To further place the RNA expression patterns found in this study in a functional context, we performed pathway analysis of the RNA-seq data with SPIA ${ }^{23}$ (see Figure 1, Supplementary Table S5 and methods 2.1) using log2 fold changes of genes that were differentially expressed between BCC and normal tissue (FDR $<0.10)$. This analysis identified three pathways as potentially activated in BCC (FDR < 0.05): cytokine-cytokine receptor interaction signaling (chemokine ( $\mathrm{C}-\mathrm{X}-\mathrm{C}$ motif) ligand 1, chemokine ( $\mathrm{C}-\mathrm{X}-\mathrm{C}$ motif) ligand 10, Interleukin 8, Chemokine (C Motif) Ligand 2, inhibin beta A chain Precursor (INHBA)) (KEGG hsa04060), chemokine signaling (Chemokine) (KEGG hsa04062) and Toll-like receptor signaling (Osteopontin, Interferon gamma-induced protein 10, Interleukin 8) (KEGG hsa04620). One pathway is potentially inhibited (Amoebiasis, (chemokine (C-X-C motif) ligand 1, Actinin, Interleukin 8) KEGG hsa05146, FDR<0.05), though given the role of this pathway in infectious diseases, we considered this finding to be of no relevance in BCC. $\mathrm{SHH}$ signaling is not in the list of significantly activated pathways based on RNA-seq data. Most of the differentially expressed genes are involved in two or all three of these pathways. Only INHBA, secreted phosphoprotein 1 (SSP1) and Toll-like receptor 8 Precursor (TLR8) were unique for one of each of the respected pathways. We also performed pathway enrichment analysis of the differentially methylated genes obtained in the original gene ranking. This analysis also identified cytokine-cytokine receptor interaction signaling as affected in BCC (Supplementary Table S6). We identified several more pathways when combining the top ranked differentially expressed and methylated genes but cytokine-cytokine receptor interaction signaling was the most significant result (Supplementary Table S7). 


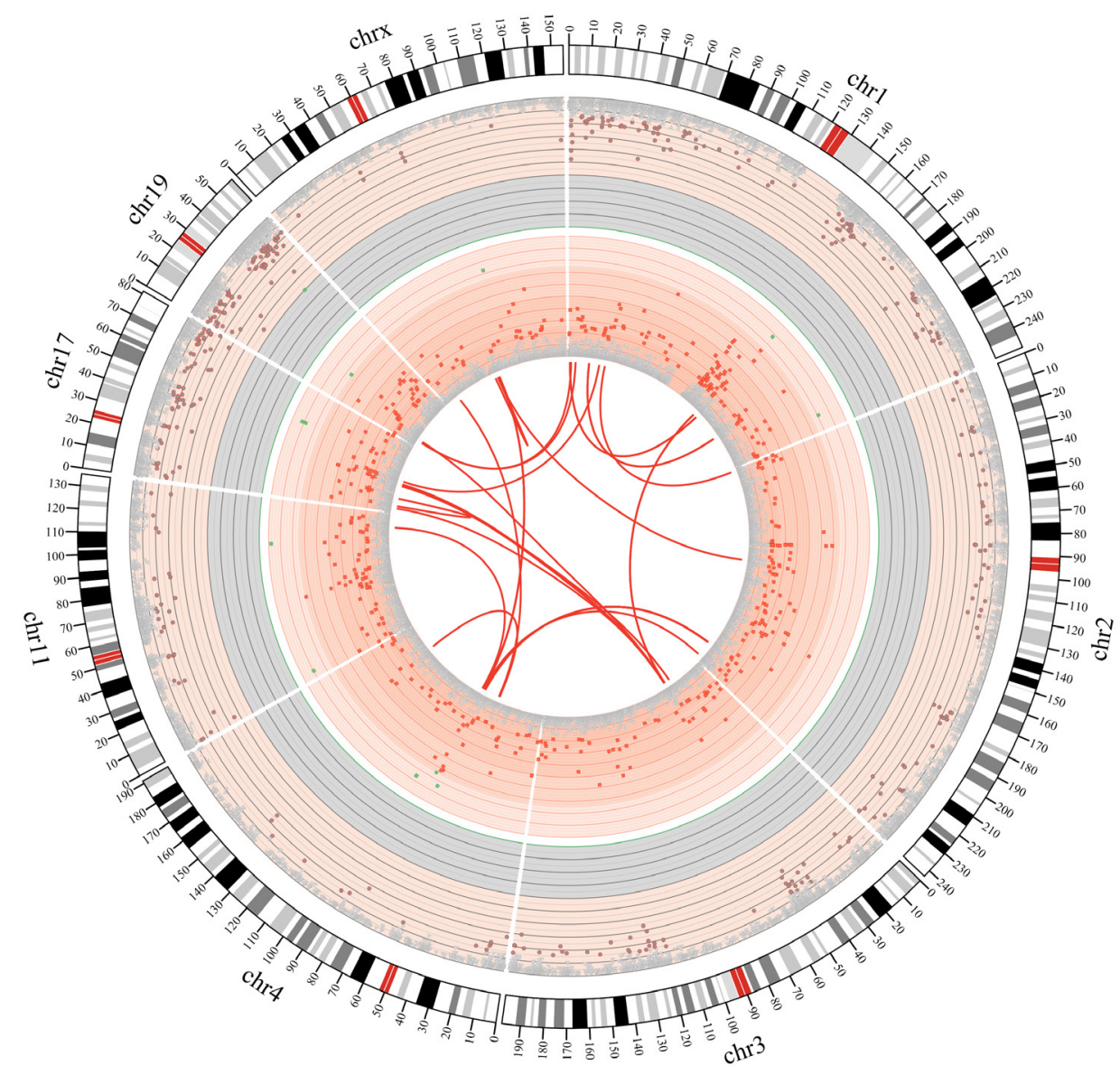

Figure 1. Circos plot.

The 'cytokine - cytokine receptor interaction' pathway was the primary candidate to emerge from our data. The central region of this plot shows the pathway ties which are potential affected by differential RNA expression in BCC (red). The circular sections from center to periphery display first the false discovery rate (FDR) for differential expression, secondly the FDR for differential DNA methylation and lastly an idiogram of the chromosomes. FDR values were log transformed and color coded by significance (green for FDR<0.05 and light grey for FDR>=0.50). Note that the axes for the FDR values are pointed towards each other. Only chromosomes with at least one significantly differentially expressed gene $(F D R<0.05)$ in the cytokine pathway were included. 
To validate the pathway analysis predictions, we performed immunohistochemical staining for INHBA, SSP1 and TLR8 on a group of 46 independent BCC samples (Supplementary Table S8). The majority of the BCC samples were positive for INHBA (61.9\%). SSP1 (56.8\%) and TLR8 (40.9\%) positivity was found less frequently. The high expression levels found for INHBA are consistent with the activation of the chemokine-signaling pathway found by SPIA. The relatively low expression levels of TLR8 are in agreement with previous work, reporting no expression of TLR8 in normal human keratinocytes ${ }^{24}$ and low levels in murine BCC cell lines. ${ }^{25}$ Of note, topically applied imidazoquinolinamines, of which imiquimod is the most widely used, are effective in the treatment of superficial BCC (sBCC) by initiating a TLR8 mediated innate-immune response. ${ }^{25}$

Our results are further substantiated by an independently conducted clinical trial in which we tested topical diclofenac, an aselective cyclooxygenase 1 and 2 inhibitor, as treatment for sBCC. After 8 weeks of twice daily application of diclofenac-3\% gel, complete tumor regression occurred in $64.3 \%$ of patients (chapter 4.1). Further support for the importance of inflammatory signaling in BCC is provided by the results of a 3 year, double blinded, randomized clinical trial in 60 patients with basal cell nevus syndrome, all harboring a germ line mutation in PTCH1. There, oral celecoxib (200 mg, twice daily, 24 months) significantly reduced BCC burden in all subjects. ${ }^{26}$ Our results suggest that $\mathrm{SHH}$ signaling might not be the sole driver of $\mathrm{BCC}$, and immune signaling seems to be important in this respect. Thus, combining $\mathrm{SHH}$-targeted therapy with (topical) anti-inflammatory drugs might increase the efficacy of non-invasive therapy.

In conclusion, we report the first comprehensive correlative analysis of the sporadic BCC methylome and transcriptome. Despite the limited number of samples analyzed, we were able to identify and confirm cytokine-cytokine receptor signaling pathway as a key mechanism in BCC. We used independent samples for MethylCap-seq and RNAseq in order to enhance the robustness of our results. However, this came at the cost of increased biologic variability. Although the MethylCap-seq and RNAseq data point in the same direction at the pathway level, strengthening the biological inference, we observe that congruent results at the gene level were often not retained at stringent FDR cut-off.

Although verification in a larger sample set is required, the combined results suggest that inflammation could be a major driver of BCC development, a notion that is supported by previous clinical work ${ }^{27}$ as well as by the results of our trial with topical application of diclofenac (unpublished data). Anti-inflammatory drugs are relatively cheap and widely available and inflammation thus could present a very attractive target for future development of drugs to treat BCC. 


\title{
Methods
}

\section{MethylCap-seq}

Sample selection and preparation

Five fresh frozen (FF) primary basal cell carcinoma samples and five control samples (FF normal sun-exposed (SE) ( $n=2)$ and sun-protected (SP) skin $(n=3))$ samples were matched for gender and age (Supplementary Table S1). DNA was extracted using the Puregene® DNA isolation kit (Gentra Systems, now Qiagen) following the manufacturer's instructions.

\section{Fragmentation and methylation enrichment}

Fragmentation was performed on a Covaris S2 to obtain fragments with an average length of 200bp. Maximum $3 \mu \mathrm{g}$ was loaded in $130 \mu \mathrm{l}$ TE in a microtube with AFA intensifier (Covaris, Woburn, Massachusetts, USA). The DNA was then analysed on the Agilent 2100 (Agilent Technologies) to check the fragment distribution on a high sensitivity DNA chip. Methylated DNA was captured using the MethylCap kit (Diagenode, Liège, Belgium). According to the manufacturer's protocol, $200 \mathrm{ng}$ of starting material was used.

\section{Library preparation, cluster generation and sequencing}

We used the DNA Sample Prep Master Mix Set 1 (New England BioLabs, Ipswich, Massachusetts, USA) in combination with the Multiplexing Sample Preparation Oligo Kit (96 samples, Illumina PE-400-1001). We used the total amount of fragmented DNA and followed the NEBNext End Repair Module Protocol. Subsequently adapters were ligated using the NEBNext Quick Ligation Module Protocol. Library size selection was performed on a $2 \%$ agarose gel (Low Range Ultra agarose Biorad, Veenendaal, the Netherlands). Fragments of $300 \mathrm{bp}+/$ - 50bp were excised and eluted on a Qiagen Gel Extraction Kit column (Qiagen).

Indexes were incorporated during the amplification step using the 'Multiplexing Sample Preparation Oligo kit' (Illumina). A paired end (PE) flow cell was prepared according to the Cluster Station User Guide. Sequencing was performed according to the GAllx user guide with a multiplexed paired-end run with $2 \times 45$ cycles.

\author{
Mapping \\ Paired-end 45bp sequence reads were mapped using bowtie (v0.12.7) software \\ 28. Only unique paired reads were retained and both fragments were required to lo- \\ cate within $400 \mathrm{bp}$ of each other on the human reference genome (GRCh37/hg 19). For \\ each sample duplicate paired reads with the exact same location were discarded.
}




\section{Data summarization and normalization}

The mapped reads were summarized using the Map of the Human Methylome (http:// www.biobix.be/map-of-the-human-methylome/mhm-version-2/). This location map contains ca. 1.5 million putatively independently methylated regions i.e. "methylation cores". For each sample, the maximum coverage within a specific methylation core was used as intensity value for that locus. Normalization factors were calculated using the Trimmed Median of M-values (TMM) method described in Robinson \& Oshlack $^{29}$ with standard settings as implemented in the edgeR package (version 2.0.3). ${ }^{30}$

\section{Differential methylation analysis}

For the analyses presented in this research we limited our focus to those loci which were contained within the exons of annotated genes or their promoter region (-2000 bp to +500 bp relative to the TSS). The remaining loci were analyzed using the R statistical package "baySeq". ${ }^{31}$ We applied a selection to the methylation cores to be analyzed by imposing additional requirements that were independent of the sample grouping (see Supplementary Methods). Briefly, in our first approach we only considered those methylation cores for which the number of unmethylated samples varied between 3 and 7. Analysis settings were identical to those in the RNA results based approach. In a second approach, methylation cores were retained for this analysis only if they were located in genes with a differential expression FDR $<0.5$ and had at least 4 read pairs across all samples.

\section{Total RNA Sequencing}

\section{Sample selection and preparation}

FF BCC samples containing more than $75 \%$ tumor cells as evaluated on haematoxylin and eosin stained sections were selected $(n=3)$. FF normal skin samples $(n=3)$ were matched with regard to gender and age. Total RNA was isolated using the standard procedure for TRIzol® RNA extraction (Invitrogen, now Life Technologies, Carlsbad, California, USA) and stored at $-80^{\circ} \mathrm{C}$. Patient characteristics are listed in Supplementary Table S1. The extracted RNA was treated with the "DNA free kit" (Ambion, Life Technologies) according to the manufacturer's instructions to remove any remaining DNA (50 $\mu$ reaction volume).

\section{rRNA depletion, Reverse Transcription and Adaptor ligation}

The extracted RNA were treated with the Ribo-Zero Gold kit (non-magnetic, Epicentre, Madison, Wisconsin, USA). Reverse transcription and adaptor ligation was performed using the ScriptSeq v2 RNA-Seq Library Preparation Kit (Epicentre). The final library was purified with AMPureXP beads (Beckman Coulter, Brea, California, USA). 


\section{Cluster generation and Sequencing}

Single read cluster generation was performed on the Cluster Station with the TruSeq v5 kit according to the manufacturer's guidelines (Illumina). All samples were sequenced for 72 cycles on a single Illumina GAll x run with one sample per lane (no multiplexing) and a Phi-X control lane.

\section{Read mapping}

To obtain expression estimates for all known human transcripts we applied Tophat (v 2.0.4) to map reads to the human genome with reference annotation GRCh37.68. Directional information obtained due to the ScriptSeq 2 library preparation was preserved in the mapping.

\section{Data summarization, normalization and differential expression analysis}

The mapped reads were summarized by counting the number of reads that mapped (at least partially within known exons and matched the sense of the transcript. The counts were again normalized by the TMM method. Differential expression was assessed using the R statistical package "baySeq".

\section{Pathway analyses}

RNA-seq based pathway analysis was performed with SPIA standard settings as implemented in R. ${ }^{23}$ MethylCap-seq based pathway analysis was performed with WebGestalt available at "http://bioinfo.vanderbilt.edu/webgestalt/". This analysis was performed twice: once with the differentially methylated genes and a second time using the union of genes in the previously mentioned list and the list of differentially expressed genes.

\section{Immunohistochemistry}

For a description of the immunohistochemical staining procedure see Suppl. methods 3.1. 


\section{References}

1. Madan V, Lear JT, Szeimies RM. Non-melanoma skin cancer. Lancet 2010;375:673-85.

2. De Meyer T, Mampaey E, Vlemmix M, et al. Quality evaluation of methyl binding domain based kits for enrichment DNA-methylation sequencing. PloS one 2013;8:e59068.

3. Anglim PP, Galler JS, Koss MN, et al. Identification of a panel of sensitive and specific DNA methylation markers for squamous cell lung cancer. Molecular cancer 2008;7:62.

4. Rausch MP, Hastings KT. GILT modulates CD4+ T-cell tolerance to the melanocyte differentiation antigen tyrosinase-related protein 1. The Journal of investigative dermatology 2012;132:154-62.

5. Holterhues C, Vries E, Louwman MW, Koljenovic S, Nijsten T. Incidence and trends of cutaneous malignancies in the Netherlands, 1989-2005. The Journal of investigative dermatology 2010;130:1807-12.

6. Zoccali G, Pajand R, Papa P, Orsini G, Lomartire N, Giuliani M. Giant basal cell carcinoma of the skin: literature review and personal experience. Journal of the European Academy of Dermatology and Venereology : JEADV 2012;26:942-52.

7. Bath-Hextall FJ, Perkins W, Bong J, Williams HC. Interventions for basal cell carcinoma of the skin. Cochrane database of systematic reviews (Online) 2007:CD003412.

8. Kasper M, Jaks V, Hohl D, Toftgard R. Basal cell carcinoma - molecular biology and potential new therapies. The Journal of clinical investigation 2012;122:455-63.

9. Jayaraman SS, Rayhan DJ, Hazany S, Kolodney MS. Mutational landscape of basal cell carcinomas by whole-exome sequencing. The Journal of investigative dermatology 2014;134:213-20.

10. Schuebel KE, Chen W, Cope L, et al. Comparing the DNA hypermethylome with gene mutations in human colorectal cancer. PLoS genetics 2007;3:1709-23.

11. Herman JG, Baylin SB. Gene silencing in cancer in association with promoter hypermethylation. The New England journal of medicine 2003;349:2042-54.

12. Brinkhuizen T, van den Hurk K, Winnepenninckx VJ, et al. Epigenetic Changes in Basal Cell Carcinoma Affect SHH and WNT Signaling Components. PloS one 2012;7:e51710.

13. Kanellou $\mathrm{P}$, Zaravinos A, Zioga M, Spandidos DA. Deregulation of the tumour suppressor genes p14(ARF), p15(INK4b), p16(INK4a) and p53 in basal cell carcinoma. The British journal of dermatology 2009;160:1215-21.

14. van Vlodrop IJ, Niessen HE, Derks S, et al. Analysis of promoter CpG island hypermethylation in cancer: location, location, location! Clinical cancer research : an official journal of the American Association for Cancer Research 2011;17:4225-31.

15. El-Hawary AK, Yassin E, Khater A, Abdelgaber S. Expression of matrix metalloproteinase-13 and Ki-67 in nonmelanoma skin cancer in xeroderma pigmentosum and non-xeroderma pigmentosum. The American Journal of dermatopathology 2013;35:45-9.

16. Poswar FO, Fraga CA, Farias LC, et al. Immunohistochemical analysis of TIMP-3 and MMP-9 in actinic keratosis, squamous cell carcinoma of the skin, and basal cell carcinoma. Pathology, research and practice 2013;209:705-9.

17. Mills M, Yang N, Weinberger R, et al. Differential expression of the actin-binding proteins, alpha-actinin-2 and -3 , in different species: implications for the evolution of functional redundancy. Human molecular genetics 2001; 10:1335-46.

18. Ulger C, Toruner GA, Alkan M, et al. Comprehensive genome-wide comparison of DNA and RNA level scan using microarray technology for identification of candidate cancer-related genes in the HL-60 cell line. Cancer genetics and cytogenetics 2003;147:28-35.

19. Baginska J, Viry E, Berchem G, et al. Granzyme B degradation by autophagy decreases tumor cell susceptibility to natural killer-mediated lysis under hypoxia. Proceedings of the National Academy of Sciences of the United States of America 2013;110:17450-5. 
20. Rasool S, Rasool V, Naqvi T, Ganai BA, Shah BA. Genetic unraveling of colorectal cancer. Tumour biology : the journal of the International Society for Oncodevelopmental Biology and Medicine 2014;35:5067-82.

21. Yau C, Sninsky J, Kwok S, et al. An optimized five-gene multi-platform predictor of hormone receptor negative and triple negative breast cancer metastatic risk. Breast cancer research : BCR 2013;15:R103.

22. Lo BK, Yu M, Zloty D, Cowan B, Shapiro J, McElwee KJ. CXCR3/ligands are significantly involved in the tumorigenesis of basal cell carcinomas. The American journal of pathology 2010;176:2435-46.

23. Tarca AL, Draghici S, Khatri P, et al. A novel signaling pathway impact analysis. Bioinformatics 2009;25:7582.

24. Li ZJ, Sohn KC, Choi DK, et al. Roles of TLR7 in activation of NF-kappaB signaling of keratinocytes by imiquimod. PloS one 2013;8:e77159.

25. Wolff F, Loipetzberger A, Gruber W, Esterbauer H, Aberger F, Frischauf AM. Imiquimod directly inhibits Hedgehog signalling by stimulating adenosine receptor/protein kinase A-mediated GLI phosphorylation. Oncogene 2013;32:5574-81.

26. Tang JY, Aszterbaum M, Athar M, et al. Basal cell carcinoma chemoprevention with nonsteroidal anti-inflammatory drugs in genetically predisposed PTCH1+/- humans and mice. Cancer Prev Res (Phila) 2010;3:25-34.

27. Muehleisen B, Jiang SB, Gladsjo JA, Gerber M, Hata T, Gallo RL. Distinct innate immune gene expression profiles in non-melanoma skin cancer of immunocompetent and immunosuppressed patients. PloS one 2012; 7:e40754.

28. Langmead B, Trapnell C, Pop M, Salzberg SL. Ultrafast and memory-efficient alignment of short DNA sequences to the human genome. Genome biology 2009;10:R25.

29. Robinson MD, Oshlack A. A scaling normalization method for differential expression analysis of RNA-seq data. Genome biology 2010;11:R25.

30. Robinson MD, McCarthy DJ, Smyth GK. edgeR: a Bioconductor package for differential expression analysis of digital gene expression data. Bioinformatics 2010;26:139-40.

31. baySeq: Empirical Bayesian analysis of patterns of differential expression in count data. 2014. 


\section{Supplementary}

Supplementary

Supplementary

Additional excel file not provided

Supplementary

Table S3

Additional excel file not provided

Supplementary

Table S4

Additional excel file not provided

Supplementary

Supplementary

Supplementary

Supplementary

Supplementary methods

References
Table S5 SPIA pathways

Table S6 Pathway analysis differentially methylated genes

Table S7 Pathway analysis DNA and RNA

Table S8 Antibody characteristics 


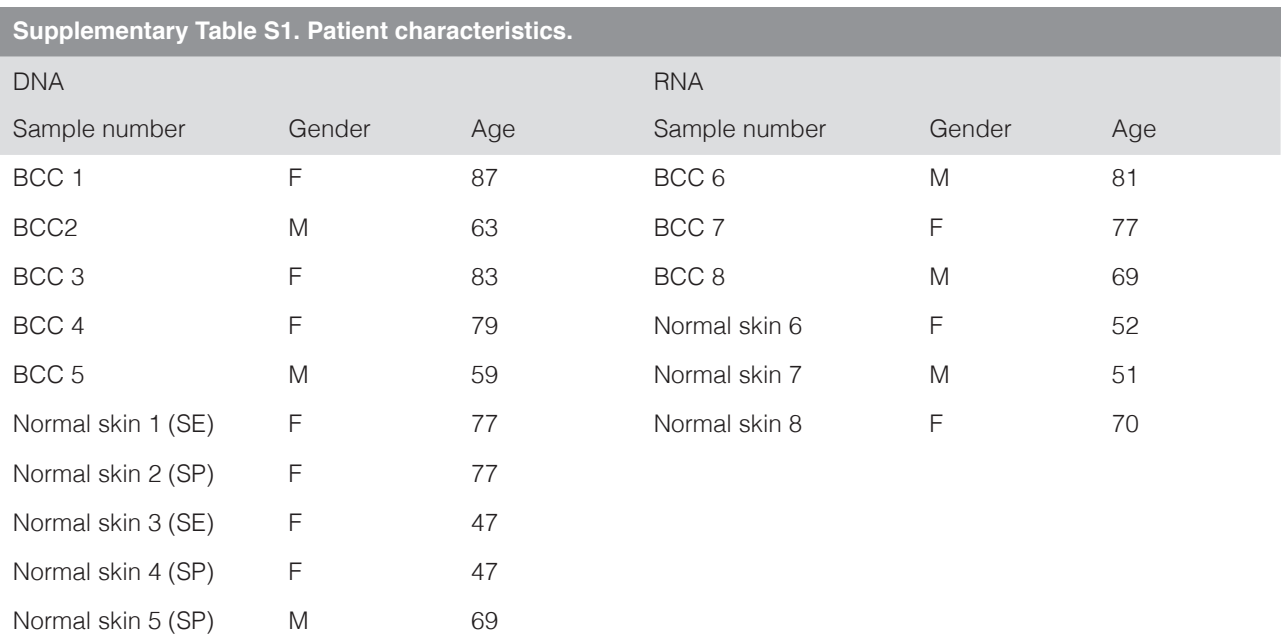

DNA: deoxyribonucleic acid, RNA: ribonucleic acid, BCC: basal cell carcinoma, SE: sun exposed, SP: sun protected.

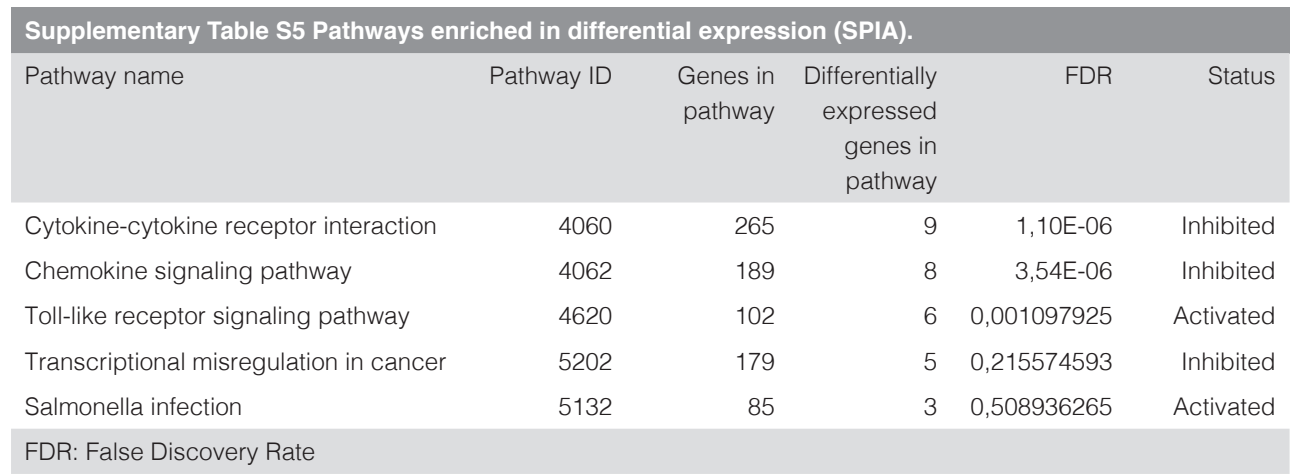

\begin{tabular}{|c|c|c|c|}
\hline \multicolumn{4}{|c|}{$\begin{array}{l}\text { Supplementary Table S6. Pathway enrichment analysis of the differentially methylated genes obtained in } \\
\text { the original gene ranking. }\end{array}$} \\
\hline Enriched KEGG pathway & Entrez IDs & Genes in pathway & FDR \\
\hline Taste transduction & 2914, 2782 & 52 & 0.01 \\
\hline P53 signaling pathway & 51246,1029 & 68 & 0.01 \\
\hline Cytokine-cytokine receptor interaction & $8784,3572,268$ & 265 & 0.01 \\
\hline Fc gamma R-mediated phagocytosis & 56848,255189 & 94 & 0.01 \\
\hline
\end{tabular}


Supplementary Table S7. Pathway enrichment analysis of genes with differentially methylation or expression.

Enriched KEGG pathway

Cytokine-cytokine receptor interaction

Toll-like receptor signaling pathway

African trypanosomiasis

Bladder cancer

Taste transduction

Pathways in cancer

Chemokine signaling pathway

p53 signaling pathway

RIG-I-like receptor signaling pathway

Fc gamma R-mediated phagocytosis

Leukocyte transendothelial migration

FDR: False Discovery Rate
Entrez IDs Genes in pathway

$8784,3627,6846,3572,268$

$7187,6696,3627$

3043,3620

4318, 1029

2914,2782

4318, 7187, 1630, 1029

2782, 3627, 6846

51246, 1029

7187,3627

56848, 255189

4318, 88
FDR

0.0026

102

0.0072

35

0.0095

42

0.0101

52

0.0104

326

0.0104

189

0.0117

68

0.0126

71

0.0126

94

0.0194

16

0.0261

Supplementary Table S8. Antibodies characteristics.

$\begin{array}{llll}\text { Antibody } & \text { Dilution } & \text { Positive control } & \text { Localization } \\ \text { INHBA } & 1: 250 & \text { Pancreas } & \text { Nucleus/cytoplasm } \\ \text { SPP1 } & 1: 500 & \text { Kidney } & \text { Cytoplasm } \\ \text { TLR8 } & 1: 1000 & \text { lung } & \text { Cytoplasm }\end{array}$

INHBA: inhibin beta A chain Precursor, SPP1: secreted phosphoprotein 1, TLR8: Toll-like receptor 8 Precursor. 


\section{Supplementary Methods}

\subsection{MethylCap-seq}

\section{Sample selection and preparation}

Five fresh frozen (FF) primary basal cell carcinoma samples and five control samples (FF normal sun-exposed (SE) $(n=2)$ and sun-protected (SP) skin $(n=3))$ samples were matched

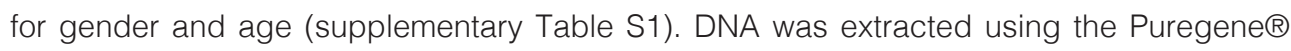
DNA isolation kit (Gentra Systems, now Qiagen) following the manufacturer's instructions.

\section{Fragmentation and methylation enrichment}

Fragmentation was performed on a Covaris S2, settings: duty cycle 10\%, intensity 5, 200 cycles per burst during $190 \mathrm{sec}$, to obtain fragments with an average length of 200bp. The power mode was frequency sweeping, temperature $6-8^{\circ} \mathrm{C}$, water level 12. Maximum $3 \mu \mathrm{g}$ was loaded in $130 \mu \mathrm{l}$ TE in a microtube with AFA intensifier (Covaris, Woburn, Massachusetts, USA). The DNA was then analyzed on the Agilent 2100 (Agilent Technologies) to check the fragment distribution on a high sensitivity DNA chip. Methylated DNA was captured using the MethylCap kit (Diagenode, Liège, Belgium). According to the manufacturer's protocol, $200 \mathrm{ng}$ of starting material was used. The concentrations of the fragmented and captured DNA were determined on a Fluostar Optima plate reader (BMG Labtech, Offenburg, Germany) with the Quant-iTTM Picogreen $®$ dsDNA assay kit (Invitrogen, Bleiswijk, the Netherlands) on 480/520nm.

\section{Library preparation, cluster generation and sequencing}

We used the DNA Sample Prep Master Mix Set 1 (New England BioLabs, Ipswich, Massachusetts, USA) in combination with the Multiplexing Sample Preparation Oligo Kit (96 samples, Illumina PE-400-1001). We used the total amount of fragmented DNA and followed the NEBNext End Repair Module Protocol; output was cleaned with the Qiaquick PCR Purification Kit (Qiagen, Manchester, United Kingdom) and eluted in $37 \mu$ l Elution Buffer (EB). After applying NEBNext dA-tailing Module Protocol, we purified the product with a Minelute

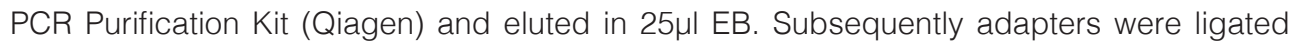
using the NEBNext Quick Ligation Module Protocol. We used the multiplexing sequencing adapters provided in the Multiplexing Sample Preparation Oligo Kit (Illumina). The ligation product was purified with the Minelute PCR Purification Kit (Qiagen) and eluted in $30 \mu \mathrm{l}$ EB. Library size selection was performed on a $2 \%$ agarose gel (Low Range Ultra agarose Biorad, Veenendaal, the Netherlands). We used a $1 \mathrm{~Kb}$ Plus ladder (Invitrogen) and ran the gel at $120 \mathrm{~V}$ for $2 \mathrm{hrs}$. A fragment of $300 \mathrm{bp}+/-50 \mathrm{bp}$ was excised and eluted on a Qiagen Gel Extraction Kit column (Qiagen), then eluted it in $23 \mu \mathrm{l}$ EB. 
Indexes were incorporated during the amplification step using the 'Multiplexing Sample Preparation Oligo kit' (Illumina). The amplified libraries were purified on a Qiaquick PCR Purification column (Qiagen 28101), eluted in $50 \mu \mathrm{l}$ EB 1:5 diluted and concentrated in a rotary evaporator to $10 \mu \mathrm{l}$. $1 \mu \mathrm{l}$ of each purified library was analyzed on an Agilent 2100 HS DNA chip. Concentration was determined on the Agilent 2100. Samples were subsequently diluted to $15 \mathrm{nM}$ and measured with qPCR according to the 'qPCR Quantification Protocol guide' (Illumina). Phix control was used as a standard. A paired end (PE) flow cell was prepared according to the Cluster Station User Guide. Sequencing was performed according to the GAllx user guide with a multiplexed paired-end run with $2 \times 45$ cycles.

\section{Mapping}

Paired-end 45bp sequence reads were mapped using bowtie (v0.12.7) software ${ }^{1}$. Bowtie parameters were set to 0 mismatches in the seed (first 28 nucleotides). Only unique paired reads were retained and both fragments were required to locate within 400bp of each other on the human reference genome (GRCh37/hg19). The mapped PE reads allow us to give every nucleotide in the genome a coverage value. For each sample duplicate paired reads with the exact same location were discarded, as these are most likely amplified from the same DNA fragment.

\section{Data summarization and normalization}

The mapped reads were summarized using the Map of the Human Methylome (http://www. biobix.be/map-of-the-human-methylome/mhm-version-2/). This location map contains ca. 1.5 million putatively independently methylated regions (200-300bp), i.e. "methylation cores". For each sample, the maximum coverage within a specific methylation core was used as intensity value for that locus. To compensate for artificial differences in read distributions, the original library sizes were multiplied by additional normalization factors calculated using the Trimmed Median of M-values (TMM) method described in Robinson \& Oshlack ${ }^{2}$ with standard settings as implemented in the edgeR package (version 2.0.3). ${ }^{3}$

\section{Differential methylation analysis}

For the analyses presented in this research we limited our focus to those loci which were contained within annotated genes or their promoter region (-2000 bp to $+500 \mathrm{bp}$ relative to the TSS). The remaining loci were analyzed using the R statistical package "baySeq". ${ }^{4}$ This package assigns likelihoods of differential expression in RNA sequencing experiments and is also suited to determine differential methylation. ${ }^{5}$ Expression data is typically featured by fewer variables and higher coverage per variable than enrichment data, causing the analysis to be prohibitively stringent. To compensate for this property of the analysis tool, we applied a selection to the methylation cores to be analyzed by imposing additional requirements that were independent of the sample grouping. In our first approach the selection criteria were aimed at studying methylation independent of the RNA results without inflating the search space too much. Using a zero count (unmethylat- 
ed) versus non-zero count (methylated) classification, we only considered those methylation cores for which the number of unmethylated samples varied between 3 and 7 , the sum of read pairs across all 10 samples was 10 or greater and the location was in the promoter region or an exon. Analysis settings were identical to those in the RNA results based approach. In a second approach we utilized the information gained in the RNA-seq experiment. Methylation cores were retained for this analysis only if they were located in genes with a differential expression FDR $<0.5$ and had at least 4 read pairs across all samples. Further, standard settings were used with 5 -fold sampling for prior estimation and 15 bootstraps for posterior estimation.

\subsection{Total RNA Sequencing}

\section{Sample selection and preparation}

FF BCC samples containing more than $75 \%$ tumor cells as evaluated on haematoxylin and eosin stained sections were selected $(n=3)$. FF normal skin samples $(n=3)$ were matched with regard to gender and age. Total RNA was isolated using the standard procedure for TRIzol@ RNA extraction (Invitrogen, now Life Technologies, Carlsbad, California, USA) and stored at $-80^{\circ} \mathrm{C}$. Patient characteristics are listed in Supplementary Table S1. The extracted RNA was treated with the "DNA free kit" (Ambion, Life Technologies) according to the manufacturer's instructions to remove any remaining DNA (50 $\mu$ reaction volume).

\section{rRNA depletion, Reverse Transcription and Adaptor ligation}

The extracted RNA were treated with the Ribo-Zero Gold kit (non-magnetic, Epicentre, Madison, Wisconsin, USA) to deplete the ribosomal RNA according to the manufacturer's specifications. The remaining RNA was purified with the RNA clean and concentrator kit (procedure > 17 nt, Zymo Research, Irvine, California, USA) and eluted in $10 \mu$ UltraPure water. The reverse transcription and adaptor ligation was performed using the ScriptSeq v2 RNASeq Library Preparation Kit (Epicentre). The initial cDNA was purified with the Minelute PCR Purification Kit (Qiagen 28004, Qiagen, Hilden, Germany). The final library was purified with AMPureXP beads (Beckman Coulter, Brea, California, USA). Quality control of the libraries was performed on a High sensitivity chip (Agilent Technologies, Santa Clara, California, USA). The concentration was determined with qPCR according to the 'qPCR Quantification Protocol guide' (Illumina, San Diego, California, USA). Phi-X control was used as a standard.

\section{Cluster generation and Sequencing}

After denaturation with $\mathrm{NaOH}$ (end concentration $0.1 \mathrm{M}$ ), we further diluted the samples to $11 \mathrm{pM}$. Single read cluster generation was performed on the Cluster Station with the TruSeq v5 kit according to the manufacturer's guidelines (Illumina). All samples were sequenced for 72 cycles on a single Illumina GAllx run with one sample per lane (no multiplexing) and a Phi-X control lane. 


\section{Read mapping}

To obtain expression estimates for all known human transcripts we applied Tophat (v 2.0.4) to map reads to the human genome. The reference annotation GRCh37.68 from the Ensembl ftp server was provided in gtf format using the "-G" option. The other parameters used were: "--no-novel-juncs" turns off search for unannotated splice junctions, "-m 1" allows 1 mismatch in seed, "-a 10" 10 base pair overhang for mapping over junctions, "-i 40" indicates the minimum length for an intron is $40 \mathrm{bp}$, "--no-coverage-search", "--microexon-search", "--segment-length 24" attempts to map each unmapped read as 3 separate reads of length $24 \mathrm{bp}$, "--min-segment-intron 40" minimum intron length for segment mapping is also $40 \mathrm{bp}$ and "--library-type fr-secondstrand" to indicate the reads contain directional information due to the ScriptSeq v2 library preparation.

\section{Data summarization, normalization and differential expression analysis}

The mapped reads were summarized by counting the number of reads that mapped (at least partially) within the exons and matched the sense of the annotated gene models. The counts were again normalized by the TMM method. Differential expression was assessed using the $R$ statistical package "baySeq". Two models of interest were defined: equal expression across all samples and differential expression between BCC samples and healthy controls. Genes that did not have at least one read in one of the samples were excluded from the analysis. Standard settings were used with these exceptions: prior estimation was performed by sampling 5 -fold over the number of remaining genes and posterior estimation was repeated in 20 bootstraps.

\subsection{Pathway analysis}

RNA-seq based pathway analysis was performed with SPIA standard settings as implemented in R. ${ }^{6}$ As input we used the Entrez gene identifiers and log fold-changes of all genes, which were found to be differentially expressed with FDR $<0.2$. MethylCap-seq based pathway analysis was performed with WebGestalt available at "http:// bioinfo.vanderbilt.edu/webgestalt/". As input we used the Entrez identifiers of all genes containing at least one differentially methylated methylation core (FDR $<0.2)$ obtained by the selecting loci with 3 to 7 unmethylated samples. This analysis was performed a second time using the union of genes in the previously mentioned list and the list of differentially expressed genes. In both cases enrichment was calculated relative to the entire human genome based on the KEGG database using the hypergeometric test with Benjamini-Hochberg correction and limiting the results to those pathways with a FDR $<0.05$ and at least 2 affected genes.

\subsection{Immunohistochemical analyses}

Forty-six independent formalin-fixed and paraffin embedded (FFPE) BCC excision specimens were selected from the archives of the Department of Pathology of the Maastricht University Medical Centre. Collection, storage and use of all tissues and patient data were performed in 
agreement with the "Code for Proper Secondary Use of Human Tissue in the Netherlands". All of the used samples and corresponding data were de-linked and anonymized. Usage of tissue samples was approved by the Maastricht Pathology Tissue Collection (MPTC) scientific committee (MPTC 2009-05). Five $\mu \mathrm{m}$ sections were mounted on superfrost plus slides and heated for $15 \mathrm{~min}$ at $70^{\circ} \mathrm{C}$. After deparaffinization the endogenous peroxidase activity was blocked for $30 \mathrm{~min}$ in $3 \% \mathrm{H} 2 \mathrm{O} 2 /$ methanol. Antigen retrieval was performed by microwave treatment at $90 \mathrm{~W}$ for 10 minutes in $10 \mathrm{mM}$ citrate buffer $(\mathrm{pH} \mathrm{6})$. Blocking of non-specific binding and diluting of primary antibodies was performed in $3 \%$ bovine-serum-albumin (BSA) in phosphate buffer saline (PBS). Sections were incubated o/n at $5^{\circ} \mathrm{C}$ with primary antibodies listed in Supplementary Table S8. The secondary antibody from the Dako Real Envision detection system (Dako, Heverlee, Belgium) was used (30min RT) and bound antibody was visualized by using the DAKO DAB chromogen (10 min). Tissue was counterstained with Gill II haematoxylin, dehydrated and coverslipped. In all reactions, appropriate positive and negative controls were included and always showed the expected results. A trained resident of the Dermatology department manually quantified the percentage of positive tumor cells. 


\section{References}

1. Langmead B, Trapnell C, Pop M, Salzberg SL. Ultrafast and memory-efficient alignment of short DNA sequences to the human genome. Genome biology 2009;10:R25.

2. Robinson MD, Oshlack A. A scaling normalization method for differential expression analysis of RNA-seq data. Genome biology 2010;11:R25.

3. Robinson MD, McCarthy DJ, Smyth GK. edgeR: a Bioconductor package for differential expression analysis of digital gene expression data. Bioinformatics 2010;26:139-40.

4. baySeq: Empirical Bayesian analysis of patterns of differential expression in count data. 2014.

5. Jones PA. Functions of DNA methylation: islands, start sites, gene bodies and beyond. Nature reviews Genetics 2012;13:484-92.

6. Tarca AL, Draghici S, Khatri P, et al. A novel signaling pathway impact analysis. Bioinformatics 2009;25:7582. 



\title{
Chapter 2.3
}

Locally advanced basal cell carcinoma has a distinct methylation and transcriptomic profile

\author{
Brinkhuizen T, van Geel M, Denil SLIJ, de Meyer T, \\ Kelleners-Smeets NWJ, Lohuis PJFM, Hoebers FJP, \\ Winnepenninckx VJL, van Steensel MAM, Mosterd K.
}

Experimental Dermatology 2016 Apr;25(4):316-8 


\section{Background}

Sporadic basal cell carcinoma (BCC) is the most frequent malignant tumor in Caucasians. Generally, BCC grow slowly and rarely metastasize or are fatal. ${ }^{1}$ Depending on tumor size, histological subtype ('infiltrative', 'micronodular' and 'morphoeic'), tumor localization (mostly face) and perineural spread, some BCC are considered high-risk. ${ }^{2}$ Those, and neglected or inadequately treated $\mathrm{BCC}$, have a greater potential of progressing to locally advanced BCC (laBCC). ${ }^{2}$ laBCC are at least $5.0 \mathrm{~cm}$ in maximum dimension, show deep tissue invasion, may metastasize and cause substantial morbidity or even mortality. ${ }^{3}$ Since laBCC are histologically indistinguishable from common BCC, they are classified according to phenotypic characteristics as described above.

Essentially all BCC have activated Hedgehog $(\mathrm{HH})$ pathway signaling due to mutations in its component genes ${ }^{1}$, however the molecular basis for the aggressive behavior of laBCC remains to be elucidated. The partial response of established laBCC to vismodegib (Erivedge), an orally active synthetic SMOOTHENED (SMO)-inhibitor, suggests that other pathways and mechanisms might be involved in their growth. ${ }^{4}$ As most, if not all, cancers are characterized by epigenetic alterations in addition to genetic changes ${ }^{5}$, this might also be the case for BCC, with epigenetic modifications contributing to its growth. The best-characterized epigenetic mechanism in carcinogenesis to date is promoter hypermethylation, comprising increased methylation of cytosines in promoter $\mathrm{CpG}$ islands and resulting in gene silencing ${ }^{5}$, for example of tumor suppressors. Others and we have previously demonstrated significant and relevant promoter hypermethylation in non-aggressive BCC (naBCC). ${ }^{6}$

\section{Question addressed}

Genome-wide BCC methylation data ("methylome") and comparisons with laBCC are lacking. We now report the first complete methylome and correlated transcriptome of an laBCC, comparing it with those of naBCC. We find significant differences that might explain the more invasive behavior of laBCC.

\section{Experimental design}

A 77-year old woman presented with an ulcerating nodular and infiltrative BCC with squamous differentiation of approximately 12 centimeters in diameter, invading much of the left side of her face, including the orbit, nose and nasal cavity, maxillary bone, and cheek. The tumor had been present for 10 to 15 years (figure 1A, 1B). 

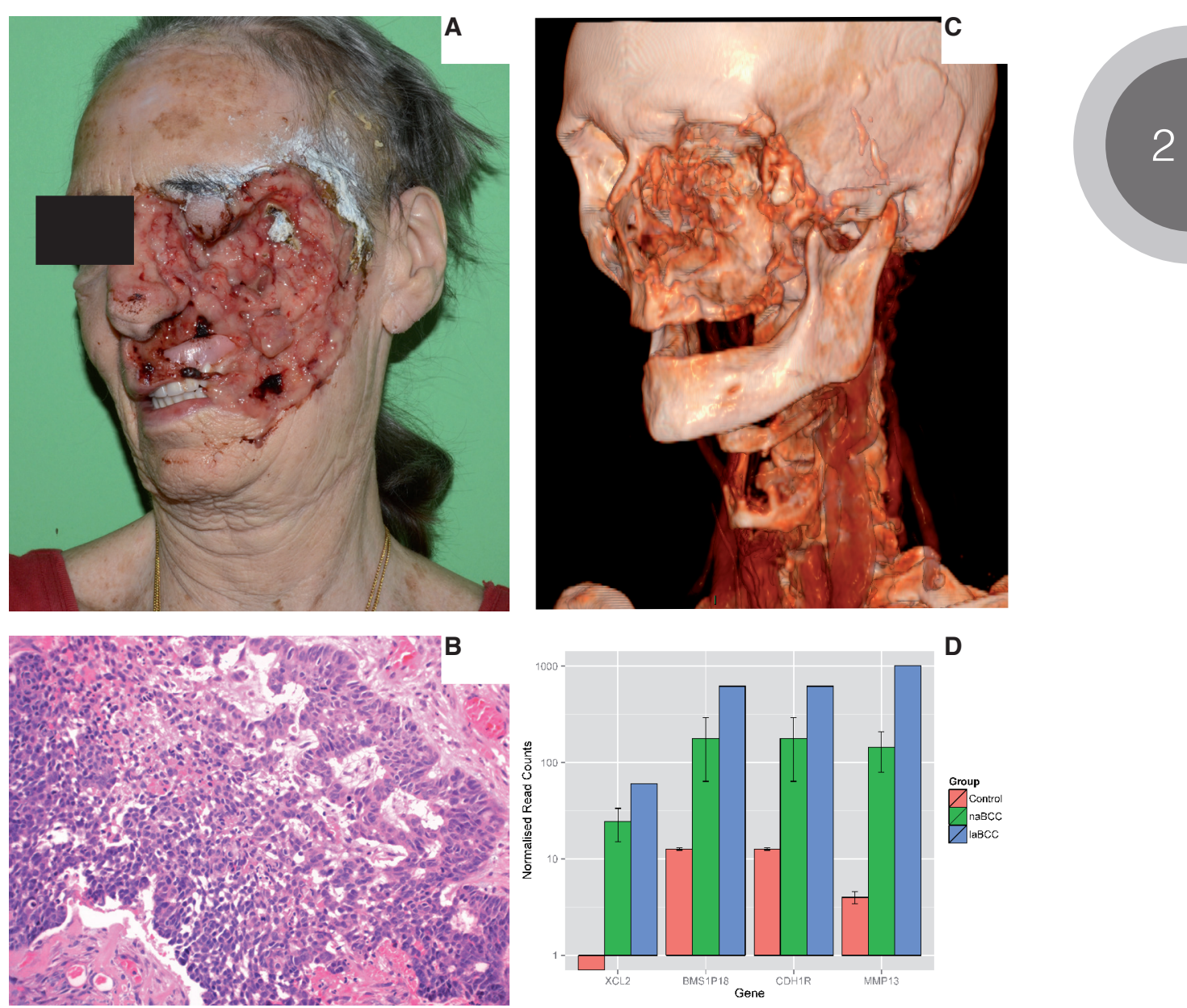

Figure 1. A locally advanced basal cell carcinoma of the face.

A. A 77-year old female with extensive facial destruction due to locally advanced BCC of the face existing for 10-15 years. The tumour was very painful and the patient had difficulties speaking and eating. Ultrasound-guided fine-needle aspiration cytology revealed a lymph node metastasis in level II of the left neck. The patient was treated by conventional excision, with a $1 \mathrm{~cm}$ margin, nasal amputation and orbital exenteration of the left eye. The resulting defect was closed with use of a free revascularized $\mathrm{m}$. rectus abdominis flap. Adjuvant locoregional radiotherapy and a cervical lymph node dissection of levels I-III were also performed. B. Haematoxolin and eosin stain of the tumour showing nests of palisading basaloid cells (20x). C. 3D reconstruction based on computed tomography (CT). CT and magnetic resonance imaging (MRI) showed an extensive tumour of $6.5 \mathrm{~cm}$ with extensive osteolysis, including the left maxillary sinus, and intraorbital growth. The left eyeball had collapsed, with lateral shifting of the optic nerve. The scans showed no intracerebral growth. D. Expression levels as normalized read counts for XCL2, BMS1P18, $\mathrm{CDH} 1 \mathrm{R}$ and MMP13. These four genes show an increased expression in this patient (locally advanced BCC) compared to other BCCs and healthy control skin. The 95\% confidence interval is indicated for the non-aggressive BCCs and healthy controls. MMP13 expression is particularly heightened, also when compared to non-advanced BCCs. 
Computed tomography (CT) and magnetic resonance imaging (MRI) showed destruction of orbit and maxilla (figure 1C). Biopsy samples were taken from both tumor tissue and unaffected skin during excision of the tumor; the patient provided her written informed consent. We sequenced the genes coding for PATCHED 1 (PTCH1), SMOOTHENED (SMO), and SUPPRESSOR OF FUSED (SUFU), all key regulators of Sonic Hedgehog (SHH) signaling and frequently mutated in BCC. Additionally, we used Methylcap-Seq ${ }^{7}$ and RNA-Seq to compare genome-wide DNA methylation and total-RNA expression patterns of this laBCC to those of naBCC and healthy skin of this and other patients (controls) that we had previously obtained. See supplementary methods S1.

\section{Results and Conclusions}

In the tumor sample we detected a heterozygous point mutation c. 1234C > T in SMO, which is predicted to result in a leucine to phenylalanine substitution at position 412 (p.(Leu412Phe)) in the fifth transmembrane domain of the SMO protein (figure S1). No deleterious changes were present in the other genes analysed. p.Leu412Phe has not yet been reported in BCC, but was previously described in medulloblastoma ${ }^{8}$, meningioma ${ }^{9}$ and maxillary ameloblastoma. ${ }^{10}$ In ameloblastoma, rare locally destructive benign tumors of odontogenic epithelium histologically resembling BCC, p.Leu412Phe appeared to be an activating mutation. ${ }^{10}$ To test whether the tumor could be an ameloblastoma instead of a laBCC, we examined BerEp4, cytokeratin (CK) 14 and CK19 expression. Both BerEp4 and CK14 (markers for BCC) showed robust staining while CK19 (marker for odontogenic epithelium) was negative. ${ }^{11,12}$ Based on these results and the histomorphological characteristics, we considered laBCC the most likely diagnosis. At the time our patient presented, vismodegib was not yet available. However, since the p.Leu412Phe SMO mutant is resistant to this SMO-inhibitor ${ }^{10}$, the tumor would not have responded.

Limiting the genome-wide DNA methylation results to those regions ( $n=15$, located in 12 genes) that were significantly differentially methylated between naBCC and controls, we found that 7 regions were more similar to naBCC in terms of sequencing coverage, while the remaining 8 resembled the controls (table 1, table S1).For the RNA data, we found that this tumor was similar to naBCC in 15 out of 20 differentially expressed genes (false discovery rate (FDR) <0.05) (table 1, table S2). In the laBCC, SMO expression was higher than in the adjacent (unaffected) skin of this patient and, interestingly, also higher compared to naBCC and the naBCC control group. Higher SMO expression levels might be an indicator of more aggressive behavior, but this notion needs to be verified in other laBCC. The most dramatic change in expression was observed for matrix metalloproteinase 13 (MMP13), where the laBCC sample showed more than 20-fold increase of expression compared to previously analysed naBCC (figure 1D). 
Table 1. Comparison of locally advanced basal cell carcinoma with respect to genes which are significantly differentially methylated and expressed between non-aggressive basal cell carcinoma and normal skin.

Differentially methylated genes

Methylation pattern similar

Methylation pattern

to naBCC

similar to normal skin

PTCH1

CKM

PTPRN2

TRDN

ATRNL1

IFI30

AC068490.2

PTPRN2

GALNT9

ADAP1

ACTN2

EDIL3

MPPED1

Differentially expressed genes

$\begin{array}{ll}\text { Expression pattern } & \text { Expression pattern } \\ \text { similar to naBCC } & \text { similar to normal skin }\end{array}$

MMP13 U3

HBB U1

XCL2 MMP9

DCC GZMK

$\begin{array}{ll}\text { SPP1 IDO1 } & \end{array}$

AMTN

BMS1P22

AL121869.1

GZMB

ACTN2

LINC00340

CDHR1

LAMP5

ZNF208

CXCL10

naBCC non-aggressive basal cell carcinoma. A single gene may contain multiple methylation sites, but is mentioned only once in the table.

Immunohistochemical analyses of MMP13 confirmed strong expression in our laBCC and an independent laBCC, compared to mild staining in naBCC (methods S1, figure S2). MMP's are a family of proteolytic enzymes, several of which promote tumor invasion by degrading the 
extracellular matrix (ECM) and basement membrane. Higher expression of MMP13 has been reported to correlate with a more invasive histological subtype in facial BCC. ${ }^{13} \mathrm{MMP} 13$ is also responsible for osteolysis in various other types of cancer, and may have therefore played a role in the extensive destruction of the maxilla in our patient. ${ }^{14}$ Our study provides the first evidence that there are substantial differences in gene methylation and expression levels between laBCC and general BCC, which could contribute to the difference in their behavior. Since there are drugs that can modulate DNA methylation, our findings may have direct therapeutic implications. Finally, the higher level of MMP13 found in laBCC, possibly combined with the higher SMO expression, might explain why laBCC are much more aggressive than naBCC. Thus, MMP13 might be used as a biomarker to distinguish BCC that should be treated and followed up more rigorously to prevent local recurrence or evolution into an laBCC. 


\section{References}

1. Madan V, Lear JT, Szeimies RM. Non-melanoma skin cancer. Lancet 2010;375:673-85.

2. Zoccali G, Pajand R, Papa P, Orsini G, Lomartire N, Giuliani M. Giant basal cell carcinoma of the skin: literature review and personal experience. Journal of the European Academy of Dermatology and Venereology : JEADV 2012;26:942-52.

3. Archontaki M, Stavrianos SD, Korkolis DP, et al. Giant Basal cell carcinoma: clinicopathological analysis of 51 cases and review of the literature. Anticancer research 2009;29:2655-63.

4. Sekulic A, Migden MR, Oro AE, et al. Efficacy and safety of vismodegib in advanced basal-cell carcinoma. The New England journal of medicine 2012;366:2171-9.

5. Herman JG, Baylin SB. Gene silencing in cancer in association with promoter hypermethylation. The New England journal of medicine 2003;349:2042-54.

6. Brinkhuizen T, van den Hurk K, Winnepenninckx VJ, et al. Epigenetic Changes in Basal Cell Carcinoma Affect SHH and WNT Signaling Components. PloS one 2012;7:e51710.

7. De Meyer T, Mampaey E, Vlemmix M, et al. Quality evaluation of methyl binding domain based kits for enrichment DNA-methylation sequencing. PloS one 2013;8:e59068.

8. Jones DT, Jager N, Kool M, et al. Dissecting the genomic complexity underlying medulloblastoma. Nature 2012;488:100-5

9. Brastianos PK, Horowitz PM, Santagata S, et al. Genomic sequencing of meningiomas identifies oncogenic SMO and AKT1 mutations. Nature genetics 2013;45:285-9.

10. Sweeney RT, McClary AC, Myers BR, et al. Identification of recurrent SMO and BRAF mutations in ameloblastomas. Nature genetics 2014;46:722-5.

11. Kato N, Endo Y, Tamura G, Motoyama T. Ameloblastoma with basal cell carcinoma-like feature emerging as a nasal polyp. Pathology international 1999;49:747-51.

12. Nagamalini BR, Suneela S, Narayan TV, et al. Origin of ameloblastoma from Basal cells of the oral epithelium- establishing the relation using neuroectodermal markers. Journal of clinical and diagnostic research : JCDR 2014;8:ZC44-7.

13. Ciurea ME, Cernea D, Georgescu CC, et al. Expression of CXCR4, MMP-13 and beta-catenin in different histological subtypes of facial basal cell carcinoma. Romanian journal of morphology and embryology = Revue roumaine de morphologie et embryologie 2013;54:939-51.

14. Wu Z, Yin H, Liu T, et al. MiR-126-5p regulates osteoclast differentiation and bone resorption in giant cell tumor through inhibition of MMP-13. Biochemical and biophysical research communications 2014;443:944-9. 


\section{Supplementary methods}

\section{MethylCap-seq}

\section{Sample selection and preparation}

Six fresh frozen (FF) primary basal cell carcinoma samples and five control samples (FF normal sun exposed (SE) $(n=2)$ and sun protected (SP) skin $(n=3))$ samples were matched with regard to gender and age. DNA was extracted using the Puregene $®$ DNA isolation kit (Gentra Systems, now Qiagen) following the manufacturer's instructions.

\section{Fragmentation and methylation enrichment}

Fragmentation was performed on a Covaris S2, settings: duty cycle $10 \%$, intensity 5, 200 cycles per burst during $190 \mathrm{sec}$, to obtain fragments with an average length of 200bp. The power mode was frequency sweeping, temperature $6-8^{\circ} \mathrm{C}$, water level 12. Maximum $3 \mu \mathrm{g}$ was loaded in $130 \mu \mathrm{l}$ TE in a microtube with AFA intensifier (Covaris, Woburn, Massachusetts, USA). The DNA was then analysed on the Agilent 2100 (Agilent Technologies) to check the fragment distribution on a high sensitivity DNA chip. Methylated DNA was captured using the MethylCap kit (Diagenode, Liège, Belgium). According to the manufacturer's protocol, $200 \mathrm{ng}$ of starting material was used. The concentrations of the fragmented and captured DNA were determined on a Fluostar Optima plate reader (BMG Labtech, Offenburg, Germany) with the Quant-iTTM Picogreen $®$ dsDNA assay kit (Invitrogen, Bleiswijk, the Netherlands) on 480/520nm.

\section{Library preparation, cluster generation and sequencing}

We made a minor modification to the 'multiplexed paired end ChIP protocol' (Illumina). We used the DNA Sample Prep Master Mix Set 1 (New England BioLabs, Ipswich, Massachusetts, USA) in combination with the Multiplexing Sample Preparation Oligo Kit (96 samples, Illumina PE-400-1001). We used the total amount of fragmented DNA and followed the NEBNext End Repair Module Protocol; output was cleaned with the Qiaquick PCR Purification Kit (Qiagen, Manchester, United Kingdom) and eluted in $37 \mu$ l Elution Buffer (EB). After applying NEBNext dA-tailing Module Protocol, we purified the product with a Minelute PCR Purification Kit (Qiagen) and eluted in $25 \mu$ l EB. Subsequently adapters were ligated using the NEBNext Quick Ligation Module Protocol. We used the multiplexing sequencing adapters provided in the Multiplexing Sample Preparation Oligo Kit (Illumina). The ligation product was purified with the Minelute PCR Purification Kit (Qiagen) and eluted in $30 \mu \mathrm{l}$ EB.

Library size selection was performed on a $2 \%$ agarose gel (Low Range Ultra agarose Biorad, Veenendaal, the Netherlands). We used a $1 \mathrm{~Kb}$ Plus ladder (Invitrogen) and ran the gel at $120 \mathrm{~V}$ for $2 \mathrm{hrs}$. A fragment of $300 \mathrm{bp}+/-50 \mathrm{bp}$ was excised and eluted on a Qiagen Gel Extraction Kit column (Qiagen), then eluted it in $23 \mu \mathrm{l}$ EB. Indexes were incorporated during the amplification step using the 'Multiplexing Sample Preparation Oligo kit' (Illumina). The amplified libraries were purified on a Qiaquick PCR 
Purification column (Qiagen 28101), eluted in $50 \mu$ EB 1:5 diluted and concentrated in a rotary evaporator to $10 \mu \mathrm{l}$. $1 \mu \mathrm{l}$ of each purified library was analysed on an Agilent 2100 HS DNA chip. The concentration was determined with smear analysis on the Agilent 2100. Samples were subsequently diluted to $15 \mathrm{nM}$ and measured with qPCR according to the 'qPCR Quantification Protocol guide' (Illumina). Phix control was used as a standard.

A paired end (PE) flow cell was prepared according to the Cluster Station User Guide. Sequencing was performed according to the GAllx user guide with a multiplexed paired-end run with $2 \times 45$ cycles.

\section{Mapping}

Paired-end 45bp sequence reads were mapped using bowtie (v0.12.7) software ${ }^{37}$. The bowtie parameters were set to 0 mismatches in the seed (first28 nucleotides). Only unique paired reads were retained and both fragments were required to locate within 400bp of each other on the human reference genome (GRCh37/hg19). The mapped PE reads allow us to give every nucleotide in the genome a coverage value. For each sample duplicate paired reads with the exact same location were discarded, as these are most likely amplified from the same DNA fragment.

\section{Data summarization and normalization}

The mapped reads were summarized using the Map of the Human Methylome (http://www. biobix.be/map-of-the-human-methylome/mhm-version-2/). This location map contains ca. 1.5 million putatively methylated regions, i.e. "methylation cores".

For each sample, the maximum coverage within a specific methylation core was used as intensity value for that locus. To compensate for artificial differences in read distributions, the original library sizes were multiplied by additional normalization factors calculated using the Trimmed Median of M-values (TMM) method described in (Robinson \& Oshlack, 2010) with standard settings as implemented in the edgeR package (version 2.0.3). ${ }^{38}$

\section{Differential methylation analysis}

For the analyses presented in this research we limited our focus to those loci which were contained within annotated genes or their promoter region $(-2000 \mathrm{bp}$ to $+500 \mathrm{bp}$ relative to the TSS). The remaining loci were analysed using the R statistical package "baySeq". ${ }^{39}$ This package was designed to assign likelihoods of differential expression in RNA sequencing experiments. It is also suited to determine differential methylation since the underlying data is based on discrete counts (negative binomial distribution). However the package is more apt at detecting quantitative differences whereas promoter methylation often has a qualitative effect on gene expression (i.e. on/off switch, see Jones 2012 for a more nuanced overview of the effects of $\mathrm{CpG}$ methylation) ${ }^{13}$. Additionally, expression data is typically featured by fewer variables and higher coverage per variable than enrichment data, causing the analysis to be prohibitively stringent. To compensate for this property of the analysis tool, we applied a selection to the methylation cores to be ana- 
lysed, by imposing additional requirements that were independent of the sample grouping. In a first approach we utilized the information gained in the RNA-seq experiment. Methylation cores were retained for this analysis only if they were located in genes with a differential expression FDR $<0.5$ and had at least 4 read pairs across all samples. Further, standard settings were used with 5 fold sampling for prior estimation and 15 bootstraps for posterior estimation. The second set of selection criteria were aimed at studying methylation independent of the RNA results without inflating the search space to much. Using a zero count (unmethylated) versus non-zero count (methylated) classification, we only considered those methylation cores for which the number of unmethylated samples varied between 3 and 7 , the sum of read pairs across all 10 samples was 10 or greater and the location was in the promoter region or an exon. Analysis settings were identical to those in the RNA results based approach.

\section{Total RNA Sequencing}

\section{Sample selection and preparation}

FF BCC samples containing more than $75 \%$ tumour cells as evaluated on haematoxylin and eosin stained sections were selected $(n=5)$. FF normal skin samples $(n=5)$ were matched with

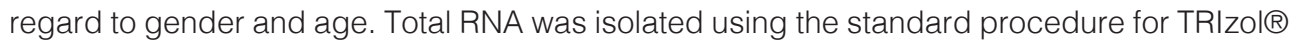
RNA extraction (Invitrogen, now Life Technologies, Carlsbad, California, USA) and stored at $-80^{\circ} \mathrm{C}$. The extracted RNA was treated with the "DNA free kit" (Ambion, Life Technologies) according to the manufacturer's instructions to remove any remaining DNA ( $50 \mu \mathrm{l}$ reaction volume).

\section{rRNA depletion, Reverse Transcription and Adaptor ligation}

The extracted RNA were treated with the Ribo-Zero Gold kit (non-magnetic, Epicentre, Madison, Wisconsin, USA) to deplete the ribosomal RNA according to the manufacturer's specifications. The remaining RNA was purified with the RNA clean and concentrator kit (procedure > 17 nt, Zymo Research, Irvine, California, USA) and eluted in $10 \mu$ UltraPure water. The reverse transcription and adaptor ligation was performed using the ScriptSeq v2 RNASeq Library Preparation Kit (Epicentre). The initial cDNA was purified with the Minelute PCR Purification Kit (Qiagen 28004, Qiagen, Hilden, Germany). The final library was purified with AMPureXP beads (Beckman Coulter, Brea, California, USA). Quality control of the libraries was performed on a High sensitivity chip (Agilent Technologies, Santa Clara, California, USA). The concentration was determined with qPCR according to the 'qPCR Quantification Protocol guide' (Illumina, San Diego, California, USA). Phix control was used as a standard.

\section{Cluster generation and Sequencing}

After denaturation with $\mathrm{NaOH}$ (end concentration 0.1 M), we further diluted the samples to 11 pM. Single read cluster generation was performed on the Cluster Station with the TruSeq v5 kit according tothe manufacturer's guidelines(Illumina). All samples were sequenced for 72 cycles on a single Illumina GAll x run with one sample per lane (no multiplexing) and a Phi-X control lane. 


\section{Read mapping}

To obtain expression estimates for all known human transcripts we applied Tophat ( $v$ 2.0.4) to map reads to the human genome. The reference annotation GRCh37.68 from the Ensembl ftp server was provided in gtf format using the "-G" option. The other parameters used were: "--no-novel-juncs" turns off search for unannotated splice junctions, "-m 1" allows 1 mismatch in seed, "-a 10" 10 base pair overhang for mapping over junctions, "-i 40" indicates the minimum length for an intron is 40 bp, "--no-coverage-search", "--microexon-search", "--segment-length 24" attempts to map each unmapped read as 3 separate reads of length $24 \mathrm{bp}$, "--min-segment-intron 40" minimum intron length for segment mapping is also $40 \mathrm{bp}$ and "--library-type fr-secondstrand" to indicate the reads contain directional information due to the ScriptSeq v2 library preparation.

\section{Data summarization, normalization and differential expression analysis}

The mapped reads were summarized by counting the number of reads that mapped within the exons and matched the sense of the annotated gene models listed in the Ensembl database of human genes (v56). Alternative assemblies of genomic regions were ignored for this purpose. The counts were again normalized by the TMM method. Differential expression was assessed using the R statistical package "baySeq". Two models of interest were defined: equal expression across all samples and differential expression between BCC samples and healthy controls. Genes that did not have at least one read in one of the samples were excluded from the analysis. Standard settings were used with these exceptions: prior estimation was performed by sampling 5 -fold over the number of remaining genes and posterior estimation was repeated in 20 bootstraps. 


\section{Immunohistochemistry}

Collection, storage and use of all tissues and patient data were performed in agreement with the "Code for Proper Secondary Use of Human Tissue in the Netherlands". We selected 5 BCC BCC, the laBCC described in this paper and an additional laBCC from the Pathologic Anatomical National Automated Archive of the Department of Pathology in the Maastricht University Medical Centre (MUMC). Normal skin samples were obtained during autopsies. Usage of tissue samples was approved by the Maastricht Pathology Tissue Collection (MPTC) scientific committee (MPTC 2009-05). Immunohistochemical stains were performed on 4- $\mu m$ formalin-fixed paraffin-embedded (FFPE) tissue sections according to standardized laboratory protocols at the Department of Pathology, MUMC+ by using a DakoAutostainer Link 48 ${ }^{\mathrm{TM}}$ (DAKO Corporation, Carpinteria, CA, USA). Briefly, after heat induced epitope retrieval (K8004 EnVision ${ }^{\mathrm{TM}}$ Flex Target Retrieval Solution High pH 9, DAKO), immunohistochemical analysis was performed using DakoAutostainer Link 48 CA, USA). Endogenous peroxidase activity was blocked by peroxidase blocking reagent for 5 minutes. The primary antibodies MMP13 (ab39012, rabbit polyclonal, 1:200 Abcam) was incubated for 20 minutes. The DAKO Envision Flex kit (K8002) was used for secondary detection (+ linker). After secondary detection, all sections were counterstained with haematoxylin. A positive control was included and showed the expected positive result. Slides were assessed by a dermato-pathologist and a trained resident dermatology and scored as mild, moderate or strong positive staining. Discrepancies were resolved by consensus.

$\begin{array}{llll}\text { Table 1. Patient characteristics immunohistochemical stains } & \\ \text { Sample } & \text { Gender } & \text { Age } & \text { Staining } \\ \text { naBCC1 } & \text { F } & 64 & \text { Mild } \\ \text { naBCC2 } & \text { F } & 74 & \text { Mild } \\ \text { naBCC3 } & \text { M } & 73 & \text { Mild } \\ \text { naBCC4 } & \text { M } & 58 & \text { Moderate } \\ \text { naBCC5 } & \text { M } & 75 & \text { Moderate } \\ \text { laBCC1 } & \text { F } & 77 & \text { Strong } \\ \text { laBCC2 } & \text { F } & 69 & \text { Strong } \\ \text { NL1 } & \text { M } & 67 & \text { Absent to mild } \\ \text { NL2 } & \text { M } & 51 & \text { Absent to mild } \\ \text { NL3 } & \text { F } & 66 & \text { Absent to mild } \\ \text { NL4 } & \text { M } & 71 & \text { Absent to mild } \\ \text { NL5 } & \text { F } & 53 & \text { Absent to mild } \\ \text { naBCC } \text { non-aggressive basal cell carcinoma, laBCC locally advanced basal cell carcinoma, F female, M male }\end{array}$



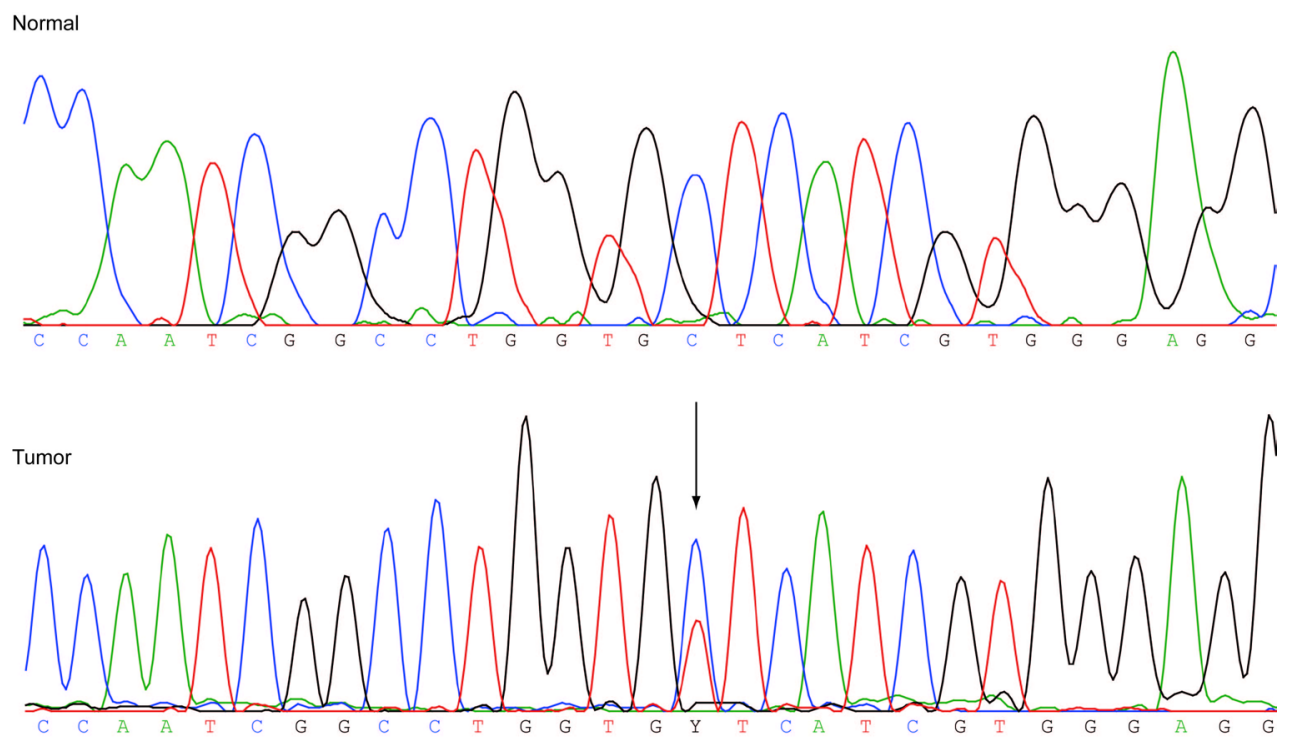

Figure S1. Sequence analysis of SMOOTHENED.

Top panel indicates normal control trace, while bottom panel shows tumour trace with the mutation indicated by an arrow (c.1234C>T).

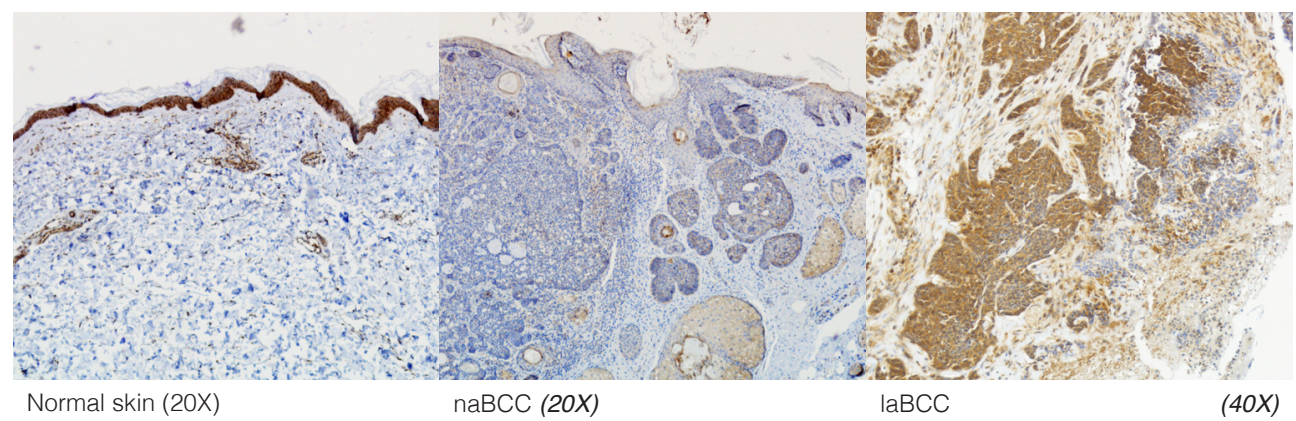

Figure S2. Immunohistochemical analysis of MMP13.

MMP13 matrix metalloproteinase 13, naBCC non-aggressive basal cell carcinoma, laBCC locally advanced basal cell carcinoma 



\section{Chapter 3}

Immunohistochemical analysis of the mechanistic target of rapamycin and hypoxia signaling pathways in basal cell carcinoma and trichoepithelioma

Brinkhuizen T, Weijzen CA, Eben J, Thissen MR, van Marion AM, Lohman BG, Winnepenninckx VJ Nelemans PJ, van Steensel MA.

PLoS One. 2014 Sep 2;9(9):e106427. 


\begin{abstract}
Background Basal cell carcinoma (BCC) is the most common cancer in Caucasians. Trichoepithelioma (TE) is a benign neoplasm that strongly resembles BCC. Both are hair follicle (HF) tumors. HFs are hypoxic microenvironments, therefore we hypothesized that hypoxia-induced signaling pathways could be involved in BCC and TE as they are in other human malignancies. Hypoxia-inducible factor 1 (HIF1) and mechanistic/mammalian target of rapamycin (mTOR) are key players in these pathways.
\end{abstract}

Objectives To determine whether HIF1/mTOR signaling is involved in BCC and TE.

Methods We used immunohistochemical staining of formalin-fixed paraffin-embedded BCC $(n=45)$ and TE $(n=35)$ samples to assess activity of HIF1, mTORC1 and their most important target genes. The percentage positive tumor cells was assessed manually in a semi-quantitative manner and categorized (0\%, < 30\%, 30-80\% and >80\%).

Results Among $45 \mathrm{BCC}$ and 35 TE examined, expression levels were respectively $81 \%$ and $57 \%$ (BNIP3), 73\% and 75\% (CAIX), 79\% and 86\% (GLUT1), 50\% and $19 \%$ (HIF1a), $89 \%$ and $88 \%$ (pAKT), 55\% and 61\% (pS6), 15\% and 25\% (pMTOR), 44\% and 63\% (PHD2) and 44\% and 49\% (VEGF-A). CAIX, Glut1 and PHD2 expression levels were significantly higher in TE when only samples with at least $80 \%$ expression were included.

Conclusions HIF and mTORC1 signaling seems active in both BCC and TE. There are no appreciable differences between the two with respect to pathway activity. At this moment immunohistochemical analyses of HIF, mTORC1 and their target genes does not provide a reliable diagnostic tool for the discrimination of BCC and TE. 


\section{Introduction}

Basal cell carcinoma (BCC) is the most common cancer in Caucasians. Its incidence is increasing by $3-10 \%$ annually, leading to a lifetime BCC risk in Caucasians of approximately 1 in 5-6. ${ }^{1}$ Sun-exposed body sites like the head and neck are most commonly affected. ${ }^{1}$ Histologically, BCC can be classified into three main subtypes: superficial, nodular and infiltrative. ${ }^{2}$ Youssef et al. were the first to show that BCC are epithelial tumors originating from progenitor cells of the interfollicular epidermis (IFE) and the upper infundibulum. ${ }^{3}$ Later evidence shows that BCC can also arise from the hair follicle (HF) bulge. ${ }^{4}$ Trichoepithelioma (TE) is a similar, though benign epithelial tumor which expresses markers that are also present in the outer root sheath of the HF ${ }^{5,6}$, which suggests that TE originate from the HF as well.

Two distinct subtypes of TE are recognized: the classic and the desmoplastic type. ${ }^{6}$ Some TE can mimic BCC macroscopically, ${ }^{6}$ making it difficult to differentiate between both tumors and this dilemma can extend to the microscopic level. Histologically, both BCC and TE are characterized by dermal nests of basaloid cells. TE differs from BCC by its absence of peripheral palisading of basaloid keratinocytes, necrosis, retraction artifact, mitotic activity and peritumoral mucin deposition. Also, TE rarely ulcerates. BCC conversely is usually not associated with formation of horn cysts and papillary mesenchymal bodies. However histological distinction is predominantly based on the higher degree of follicular differentiation as found in TE. ${ }^{6}$ Differentiation between these two HF derived tumors is important because of their distinct biologic behavior and therapeutic approach. Decreased oxygen levels are a common feature in many tumors ${ }^{7}$, including cutaneous squamous cell carcinoma and melanoma. ${ }^{8}$ While the human dermis is well-oxygenated and the epidermis is modestly hypoxic, the HF is considered as a moderately to severely hypoxic microenvironment. ${ }^{9}$ A primary mediator of hypoxia-induced gene expression in human HFs is the hypoxia-inducible transcription factor 1 (HIF1). ${ }^{10} \mathrm{HIF} 1$ is activated in several tumor types, ${ }^{11}$ and is mainly negatively regulated by prolyl hydroxylase 2 (PHD2); the key oxygen sensor. ${ }^{12}$ In hypoxic conditions, HIF1a regulates the expression of important proteins including vascular endothelial growth factor (VEGF-A), glucose transporter 1 (GLUT1), catalytic enzymes such as carbonic anhydrase IX (CAIX) and pro-apoptotic BCL-2/adenovirus E1B 19 kDa-interacting protein 3 (BNIP3). VEGF-A is suggested as a key driver in the angiogenic response and is overexpressed in most solid cancer. As a consequence, inhibition of VEGF-A can suppress tumor growth. ${ }^{13}$ GLUT1 is responsible for glucose uptake and the expression of GLUT1 increases under hypoxic conditions inducing glycolysis. ${ }^{14}$ Tumors generally have high rates of glycolysis (the Warburg effect). ${ }^{15}$ CAIX is involved in several regulatory process that are beneficial for tumour survival, the most important is $\mathrm{pH}$ regulation. Aggressive tumour behavior and poor patient outcome is associated with high CAIX expression levels. ${ }^{16}$ Also BNIP3 is associated with poor prognosis in some cancers, promoting apoptosis and even autophagy. ${ }^{17}$ Hence, all of these genes play critical roles in tumor growth. ${ }^{18}$ Of note, they can also be induced by mechanistic/mammalian target of rapamycin (mTORC1) signaling ${ }^{19}$ which is activated in several tumor types. ${ }^{20}$ Also, mTORC1 enhances the protein levels of HIF via HIF1a and consequently enhances the expression of HIF target genes. ${ }^{21}$ Dodd et al additionally showed that mTORC1 mediates VEGF-A expression via both HIF1a dependent and independent mech- 
anisms. ${ }^{19}$ Conversely, hypoxia promotes tuberous sclerosis complex 2 (TSC2) via 'regulated in development and DNA damage responses 1' (REDD1) that leads to inhibition of mTORC1 and reveals a feedback mechanism where HIF can turn off mTOR complex 1 (mTORC1). ${ }^{22}$ mTOR, as part of mTORC1, plays an integral role in the coordination of cell growth and division in response to growth factors, nutrients and the energy status of the cell (Figure 1.))..$^{22}$

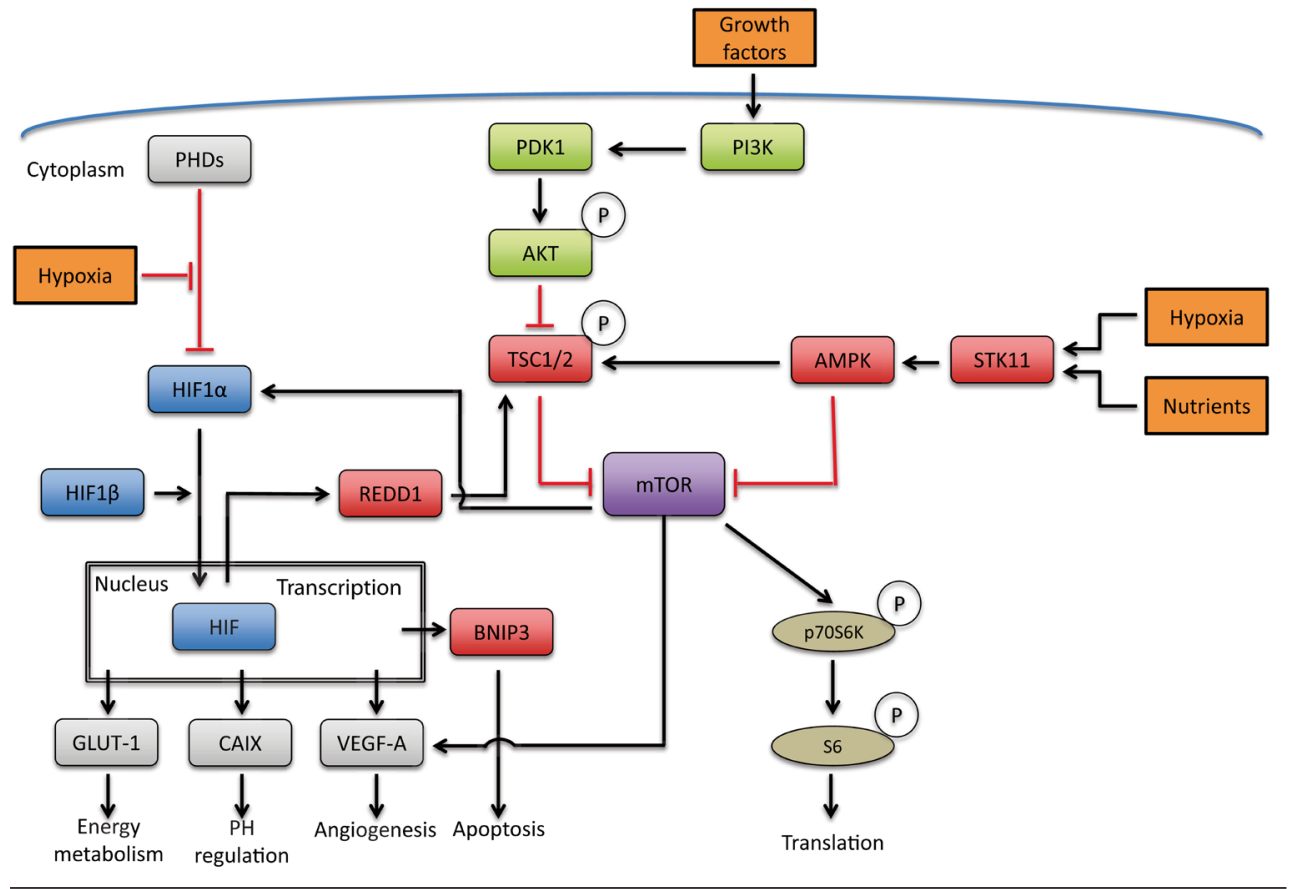

\section{Figure 1: Simplified depiction of pathways affected by hypoxia.}

Under hypoxic conditions the expression of prolyl hydroxylase domain proteins (PHDs) is reduced leading to an induction of hypoxia inducible factor $1 \mathrm{a}(\mathrm{HIF} 1 \mathrm{a})$ expression, which becomes stable and active as a transcription factor, together with hypoxia inducible factor $1 \beta$ (HIF1 $\beta)$. HIF1 activation regulates the expression of several target genes whose products address the needs of oxygen starved cells, such as vascular endothelial growth factor (VEGF-A), BCL-2/adenovirus E1B 19 kDa-interacting protein 3 (BNIP3), glucose transporter 1 (GLUT1) and glycolytic enzymes such as carbonic anhydrase IX (CAIX). Hypoxia also influences mechanistic/mammalian target of rapamycin complex 1 (mTORC1) signaling mainly mediated through hypoxic activation of the TSC1TSC2 complex by REDD1. First, phosphatidylinositol 3 kinase $(\mathrm{PI}(3) \mathrm{K})$ and protein kinase $\mathrm{B}(\mathrm{AKT})$ have been implicated in the activation of the mTOR protein kinase. One critical target of AKT that regulates mTOR is the tumour suppressor protein, tuberin (TSC2). Tuberin negatively regulates mTOR signaling, and AKT activation circumvents this inhibition. Constitutive mTOR signaling positively stimulates S6 kinase (S6K), a downstream effector of mTOR pathway, which mainly drives cell growth and proliferation. Also, mTOR enhances the protein levels of HIF and consequently enhances the expression of HIF target genes. Second, under conditions of hypoxia, intracellular ATP levels drop and AMP levels rise. AMP directly binds to a subunit of AMP activated protein kinase (AMPK), which is then phosphorylated by serine/threonine protein kinase 11 (STK11/LKB1). Elevated concentrations of AMPK can cause a complete inhibition of mTOR (mTORC1) activity without affecting PI(3)K-AKT signaling. 
Human tumors are characterized by an heterogeneous microenvironment that differs from normal tissue with respect to nutrient supply, $\mathrm{pH}$ and oxygenation. As discussed, deficiencies in oxygenation (hypoxia) is strongly associated with tumor development, growth, metastasis and poor response to therapy. HIF and mTORC1 signaling are crucial in here, either directly or indirectly acting on the hypoxia response. ${ }^{23}$

Based on the hypoxia affecting the HF and the known role of both HIF and mTORC1 in tumor growth, we have previously hypothesized that hypoxia response pathways are involved in the pathogenesis of HF tumors. ${ }^{10}$ If HIF and mTORC1 pathways do contribute to the growth of HF tumors, new opportunities for targeted therapy and diagnostics could emerge.

Therefore, we decided to examine BCC as this is the most prevalent HF tumor and included the very similar TE. ${ }^{9,10,24}$ We analysed the expression of the most important target genes in the hypoxia response using immunohistochemical stains of both $\mathrm{BCC}$ and TE. To the best of our knowledge, this is the first large series of BCC and TE to date to be analysed for HIF1 and mTORC1 activity. Our results suggest that HIF1/mTORC1 mediated signaling is active in both tumor types.

\section{Material and Methods}

\section{Tissue samples}

Collection, storage and use of all tissues and patient data were performed in agreement with the "Code for Proper Secondary Use of Human Tissue in the Netherlands". We randomly selected 45 BCC and 80 TE haematoxylin-eosin stain (HE) slides from the Pathologic Anatomical National Automated Archive of the Department of Pathology in the Maastricht University Medical Centre (MUMC). A combination of search terms was used to retrieve reports of the specific histological diagnosis. Based on the morphological classification by Rippey, BCC were subcategorized into superficial, nodular and infiltrative during routine histological diagnostic examination. ${ }^{2}$ All TE HE slides were reviewed by three independent investigators (i.e. a dermato-pathologist (AM), a general pathologist (BL) and a dermatologist (MT)), because the histopathologic diagnosis of TE can present considerably more difficulties than that of BCC. The 80 slides were reviewed based on the presence or absence of the following characteristics: tumor-stroma cleft formation, ulceration, epithelial primitive structures, small keratinous cysts, inflammatory response, mitosis, necrotic tumor cells, papillary mesenchymal bodies, stromal edema and peritumoral mucin production. ${ }^{5}$ Eventually, 35 TE were found to unambiguously fit the criteria for classic TE and these were used for the analysis, together with 45 BCC. All of the used samples and corresponding data were de-linked and anonymized. Usage of tissue samples was approved by the Maastricht Pathology Tissue Collection (MPTC) scientific committee (MPTC 2009-05). 


\section{Immunohistochemistry}

Formalin fixed and paraffin embedded (FFPE) biopsies as well as excision specimens were used. Four-micrometer sections were cut and stained with primary antibodies, listed in table 1. Hif1a, CAIX, Glut-1 and VEGF-A stains were performed on a Dako autostainer system with use of a pre-treatment module using EnVision FLEX Target Retrieval Solution, High pH (Dako, Heverlee, Belgium). The antibodies were applied for 20 minutes at room temperature. For HIF1a, the slides were additionally incubated with Envision Flex Mouse Linker to amplify the signal. The Dako Envision Flex kit (K8002) was used for secondary detection. For BNIP3, PHD2, pS6, pMTOR2448 and PAKT, slides were deparaffinized in xylene, rehydrated and incubated in 0.3\% (PHD2, pMTOR2448) or 3\% (BNIP3, pS6) hydrogen peroxide $(\mathrm{H} 2 \mathrm{O} 2)$ in methanol for 30 minutes to inactivate endogenous peroxidase activity. Antigen retrieval was performed by microwave treatment at $90 \mathrm{~W}$ for 10 minutes in $10 \mathrm{mM}$ citrate buffer ( $\mathrm{pH} 6$ ) and non-specific protein binding was blocked using 3\% bovine-serum-albumin (BSA). The sections were incubated for 1 hour at room temperature (BNIP3, PHD2) or overnight at $4^{\circ} \mathrm{C}$ (pS6, pMTOR2448, pAKT). For secondary detection an Envision detection system was used and bound antibody was visualized by using 3,3-diaminobenzidine (DAB) for 10 minutes. After secondary detection, all sections were counterstained with Gill II haematoxylin, dehydrated and coverslipped. In all reactions, appropriate positive and negative controls were included and always showed the expected positive resp. negative results.

$\begin{array}{lll}\begin{array}{l}\text { Table 1. Antibodies used for immunohistochemical analysis } \\ \text { Antibody }\end{array} & \text { Dilution } & \text { Producer } \\ \text { BNIP3 } & 1: 800 & \text { Sigma Aldrich, St. Louis, U.S.A. } \\ \text { CAIX } & 1: 1000 & \text { Novus Biologicals, Littleton, U.S.A. } \\ \text { GLUT1 } & 1: 200 & \text { Thermo Scientific, Landsmeer, the Netherlands } \\ \text { HIF1a } & 1: 50 & \text { BD Transduction Laboratories, Breda, the Netherlands } \\ \text { pAKT } & 1: 25 & \text { Cell Signaling technology, Beverly, MA, U.S.A. } \\ \text { PS6 } & 1: 50 & \text { Cell Signaling technology, Beverly, MA, U.S.A. } \\ \text { PMTOR2448 } & 1: 50 & \text { Cell Signaling technology, Beverly, MA, U.S.A. } \\ \text { PHD2 } & 1: 250 & \text { Novus Biologicals, Littleton, US } \\ \text { VEGF-A } & 1: 200 & \text { Thermo Scientific, Landsmeer, the Netherlands }\end{array}$

BCL2/adenovirus E1B 19 kDa protein-interacting protein 3 (BNIP3), carbonic anhydrase IX (CAIX), glucose transporter member 1 (GLUT1), hypoxia-inducible factor 1-alpha (HIF1a), phosphorylated -protein kinase B (pAKT), phosphorylated-S6 (pS6), phosphorylated-mechanistic target of Rapamycin (pMTOR), prolyl hydroxylase domain protein 2 (PHD2), vascular endothelial growth factor (VEGF-A). 


\section{Interpretation of the stains}

A trained medical student of the Department of Dermatology, MUMC (CW) and an experienced resident of the Department of Pathology, MUMC (JE) examined all sections. Both were blinded for patient details. Any discrepancy between the observers was discussed and resolved by consensus. For all tumors, at least four randomly chosen high-power fields (magnification 200x) per slide were assessed to determine the percentage positive tumor cells. The percentage of cell staining was scored as 0 (no staining), 1 ( $<30 \%$ staining), 2 (30-80\% staining) and 3 (>80\% staining $)^{25}$. For all assessments, the HF was used as internal standard and considered as $100 \%$ positive.

\section{Statistical analysis}

Statistical analyses were carried out using SPSS version 20.0 software (SPSS, Chicago, IL, USA). Descriptive data were presented as absolute numbers and percentages for categorical data and as means with standard deviations for continuous data. The Chi-square test for independent proportions was performed to evaluate the differences and similarities in expression of CAIX, BNIP3, GLUT1, HIF1a, pAKT, PHD2, pMTORC1, pS6 and VEGF-A between BCC and TE specimens. Correlations among the different stainings were assessed by Spearman's correlation coefficient. $P<0.05$ was considered to be statistically significant. 


\section{Results}

\section{Sample characteristics}

Forty-five BCC (superficial $n=14$, nodular $n=24$, and infiltrative $n=7$ ) were included. Among the 80 TE cases selected, 35 were unanimously found to be classic type TE. Distribution of patient and tumor characteristics within the TE and BCC group are equal and listed in table 2.

\begin{tabular}{|c|c|c|}
\hline & Trichoepithelioma & Basal cell carcinoma \\
\hline Number tumours & 35 & 45 \\
\hline Biopsy/excision* & $19 / 16(54.3 / 45.7)$ & $0 / 45(0.0 / 100.0)$ \\
\hline Gender $(\mathrm{m} / \mathrm{f})^{*}$ & $16 / 21(43.2 / 56.8)$ & $24 / 21(53.3 / 46.7)$ \\
\hline Mean patient age in years & 58.8 & 68.6 \\
\hline \multicolumn{3}{|l|}{ Tumour localisation* } \\
\hline Head & $28(80.0)$ & $18(40.0)$ \\
\hline Nek & $3(8.6)$ & $4(8.9)$ \\
\hline Trunk & $3(8.6)$ & 15 (33.3) \\
\hline Arm & $0.0(0.0)$ & $2(4.4)$ \\
\hline Leg & $1(2.9)$ & $6(13.3)$ \\
\hline \multicolumn{3}{|l|}{ TE Subtype* } \\
\hline Classic TE & $35(100.0)$ & \\
\hline Desmoplastic TE & $0(0.0)$ & \\
\hline \multicolumn{3}{|l|}{ BCC Subtype* } \\
\hline Superficial BCC & & $14(31.1)$ \\
\hline Nodular BCC & & $24(53.3)$ \\
\hline Infiltrative BCC & & $7(15.6)$ \\
\hline
\end{tabular}

Hypoxia target staining patterns in basal cell carcinoma and trichoepithelioma

We investigated the activation status of hypoxia and mTORC1 signaling cascades by immunohistochemical analysis of BCC and TE. Immunohistochemical staining of BNIP3, GLUT1, CAIX, VEGF-A and PHD2 was observed in the suprabasal portion of the overlying epidermis of the BCC and TE tumor islands and in the internal control, the HF. Immunohistochemical staining for HIF1a was negative in epidermis overlying the tumor islands, while the HF showed weak scattered positive nuclear expression. VEGF-A showed expression in the endothelial cells of blood vessels. An overview of all stains is presented in figure 2 and 3 . 
Overall, about half of all BCC and TE indicate active hypoxia signaling, however the BCC specimens tended to have less CAIX, GLUT1, and PHD2 expression than TEs (Fig. 4a). In TE high expression levels (>80\%) of PHD2, GLUT1 and CAIX were observed in $53.3 \%, 31.0 \%$ and $34.4 \%$, respectively (Fig. 4b). Conversely, HIF1a was more often positive in BCC nuclei than in TE $(50.0 \%$ vs. $19.4 \%(P<0.05))$. The downstream effectors BNIP3 and VEGF-A showed no significantly different expression levels. When examining different BCC subtypes, nodular BCC harbored more GLUT1 and PHD2 expression compared to superficial and infiltrative BCC with P-values of respectively $\mathrm{P}=0.032$ and $P=0 \cdot 17$. An overview of the expression in BCC and TE is presented in table 3 .
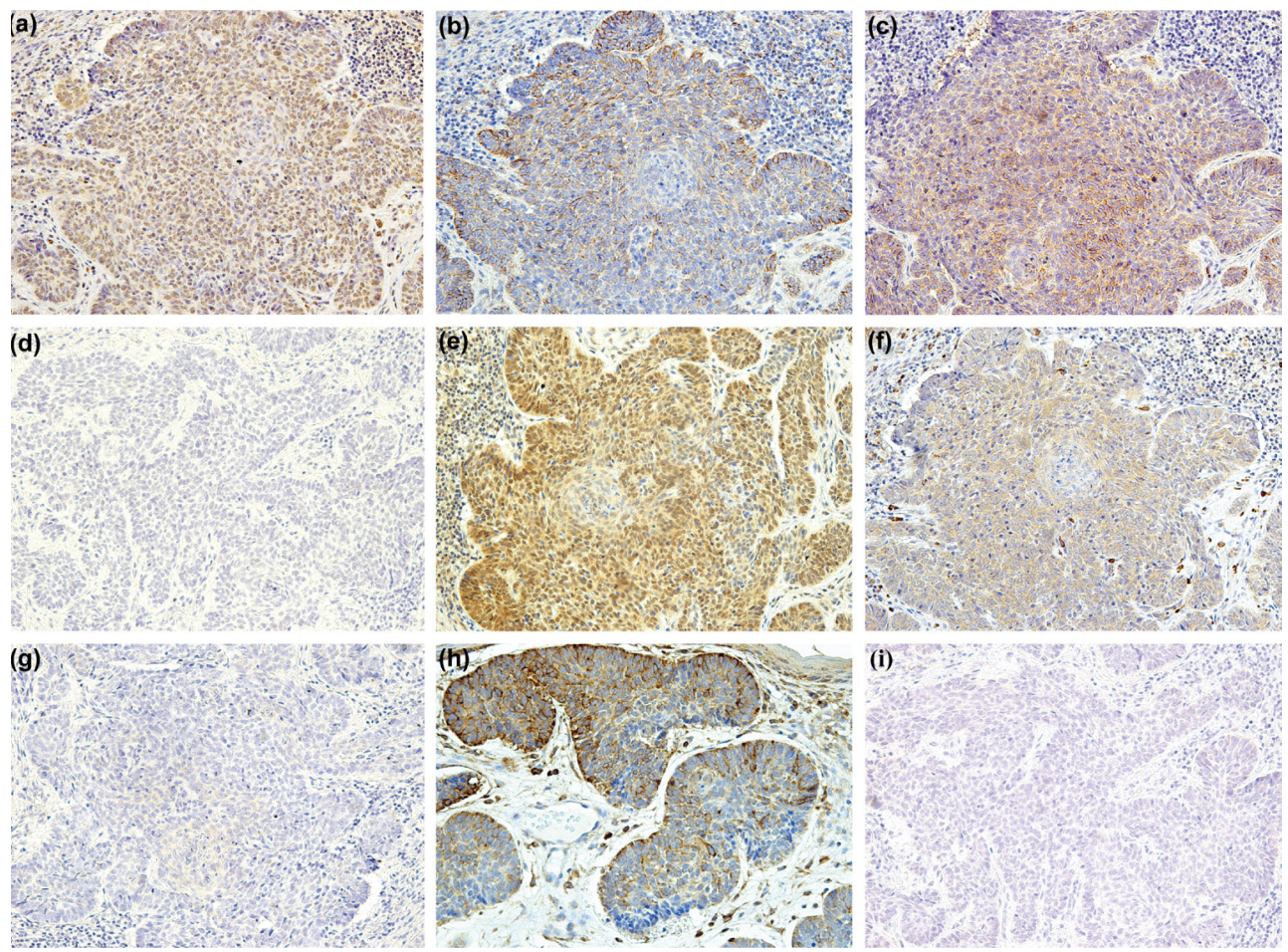

Figure 2: Immunohistochemical stains for hypoxia inducible factor 1a (HIF1a), phosphorylated mechanistic/ mammalian target of rapamycin (pmTOR) and their target genes in basal cell carcinoma (BCC).

BCL-2/adenovirus E1B 19 kDa-interacting protein 3 (BNIP3) (a); carbonic anhydrase IX (CAIX) (b); glucose transporter 1 (GLUT1) (c); HIF1a (d); phosphorylated protein kinase B (pAKT) (e);phosphorylated ribosomal protein S6 (pS6) (f); pmTOR (g); prolyl hydroxylase domain protein 2 (PHD2) (h); vascular endothelial growth factor (VEGF-A) (i). Original magnification: $(\mathrm{a}-\mathrm{i}) \times 200$. 

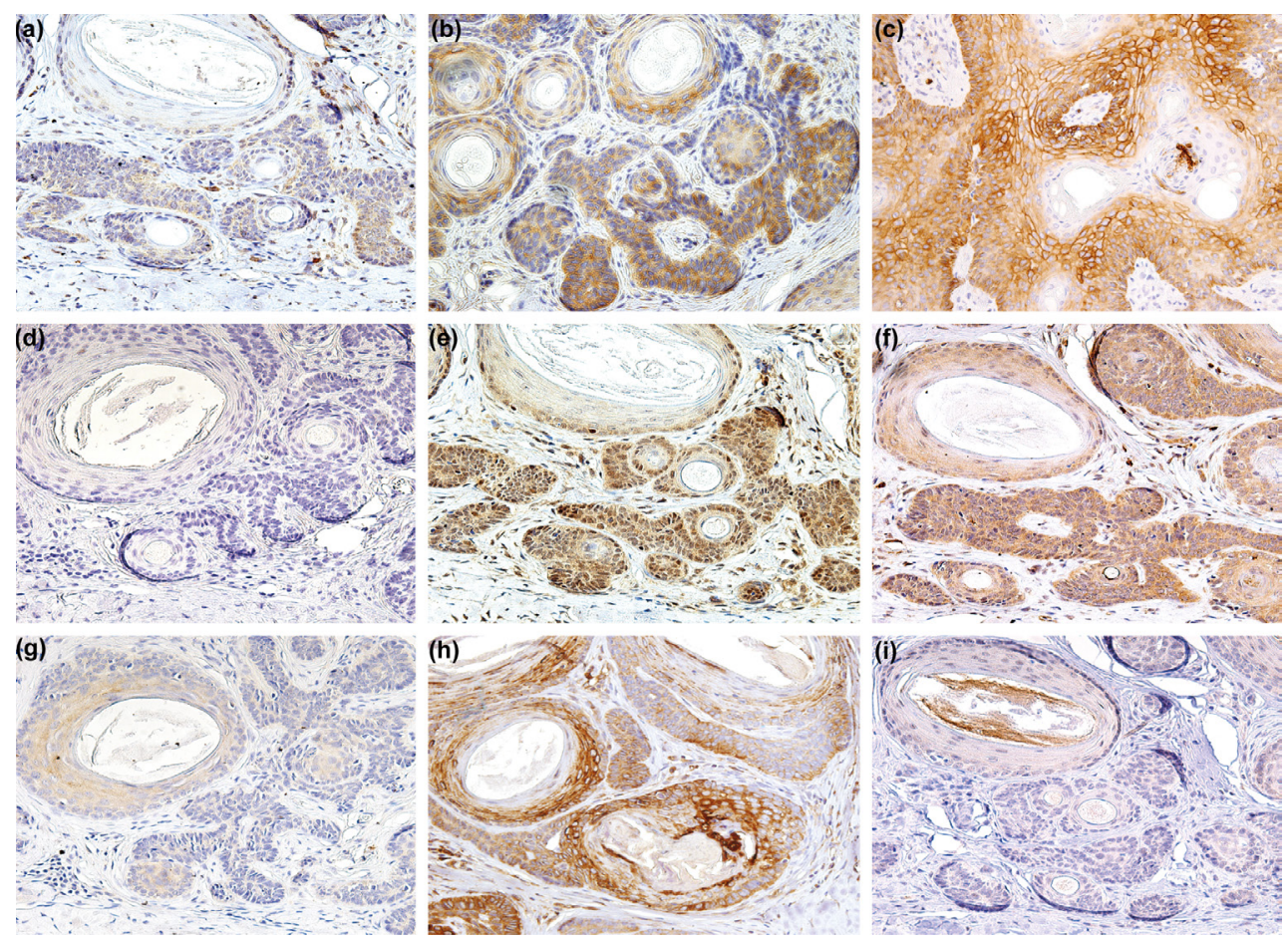

Figure 3: Immunohistochemical staining for hypoxia inducible factor 1a (HIF1a) and phosphorylated mechanistic/mammalian target of rapamycin (pmTOR) and their target genes in trichoepithelioma (TE).

BCL-2/adenovirus E1B 19 kDa-interacting protein 3 (BNIP3) (a); carbonic anhydrase IX (CAIX) (b); glucose transporter 1 (GLUT1) (c); HIF1a (d); phosphorylated protein kinase B (pAKT) (e); phosphorylated ribosomal protein S6 (pS6) (f); p-mTOR (g); prolyl hydroxylase domain protein 2 (PHD2) (h); vascular endothelial growth factor (VEGF-A)

(i). Original magnification: $(a-i) \times 200$.

\section{mTORC1 target staining patterns in basal cell carcinoma and trichoepithelioma}

An overview of all stains is shown in figure 2,3,4 and table 3. pAKT and pS6 were positive in the epidermis overlying the BCC and TE tumor islands and the HF, while immunohistochemical expression of phosphorylated mTORC1 at Ser-2448 (pmTOR) in the overlying epidermis was negative. The HF showed some weak and scattered pmTOR staining. In contrast, we found PAKT to be highly expressed in $88.9 \%$ of BCC and $87.5 \%$ in TEs $(P=0.791)$. As a downstream effector of pAKT, pmTOR exhibited positive intracytoplasmic and partly membranous expression in only $15.0 \%$ of the BCC, while $33.3 \%$ of the TE showed positive staining $(\mathrm{P}=0.083)$. pS6 showed no significant difference between intracytoplasmic staining expression in BCC (55.0\%) specimens and TE (63.3\%) specimens $(P=0.935)$. No differences among the BCC subtypes could be detected. 
Spearman's correlation coefficients among HIF and mTORC1 signaling target genes

All stains were statistically analyzed for correlation of expression patterns revealing the following significant correlations. In BCC, significant correlations between CAIX and GLUT1 $(r=0.369, P=0.024)$, CAIX and pS6 ( $r=0.522, P=0.001)$ and CAIX and PHD2 $(r=0.426, P=0.007)$ were detected as well as between PHD2 and BNIP3 ( $r=0 \cdot 485, P=0 \cdot 003)$ and PHD2 and pS6 $(r=0 \cdot 399, P=0 \cdot 012)$. Furthermore, the expression of VEGF-A correlated significantly with that of pMTOR ( $r=0.342, P=0.033)$. Moreover in TE, PHD2 was significantly correlated with CAIX $(r=0 \cdot 438, P=0.017)$ and GLUT1 ( $r=0.544, P=0.003)$. Lastly, pAKT expression correlates significantly with pS6 $(r=0.438, P=0.015)$ and VEGF-A with pMTOR $(r=0.502, P=0.07)$ in TE. 


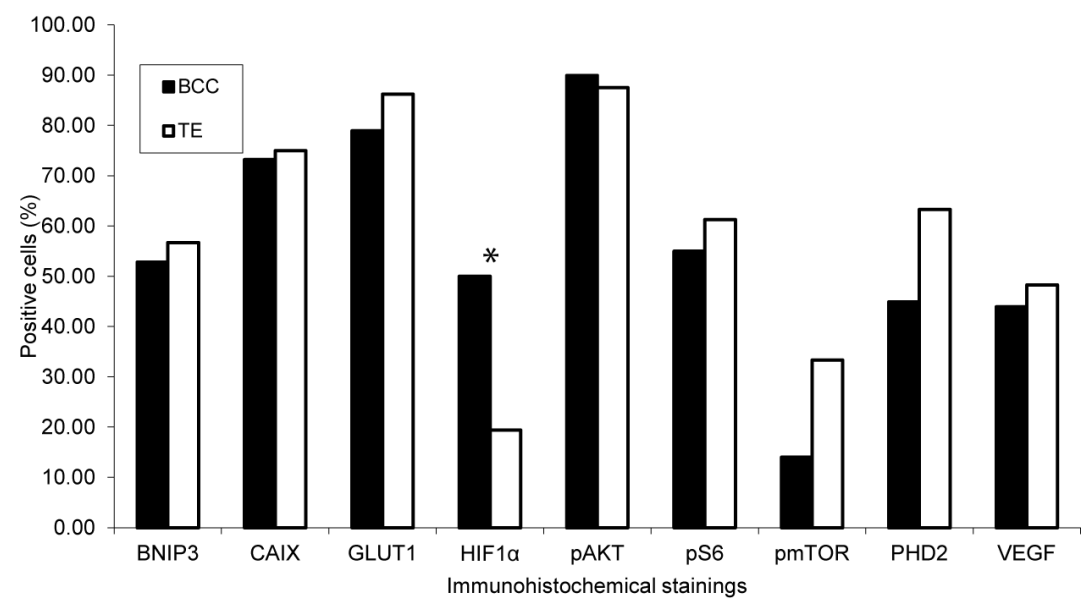

b Percentage positive excision specimens cutoff value $>80 \%$

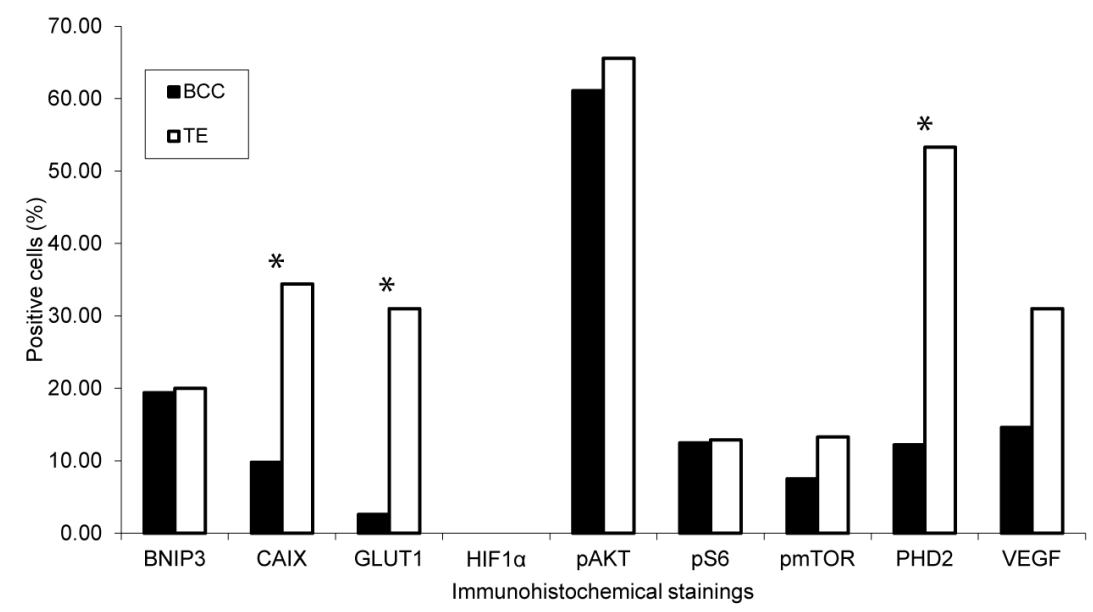

Figure 4: Percentage of positive specimens between basal cell carcinoma and trichoepithelioma.

Panel a represents all tissue samples being either positive or negative. In panel b the same results are shown, however here a cut off value of $80 \%$ of the tumour cells being positive was used. ${ }^{*}(P<0 \bullet 05)$, basal cell carcinoma (BCC); trichoepithelioma (TE). 


\section{Discussion}

The HIF and mTORC1 signaling pathways play crucial roles in many malignancies. ${ }^{23}$ To the best of our knowledge, we are the first to perform a systematic analysis of HIF and mTORC1 signaling in both BCC and TE. Although TE showed significantly more expression of CAIX, Glut1 and PHD2, this was only in the proportion of samples with high expression levels of at least $80 \%$ and thus not likely to reflect a genuine difference between the two tumor types. The correlations found between components of HIF signaling and the mTORC1 pathway are in line with known crosstalk between both pathways. ${ }^{23}$ GLUT1, CAIX and BNIP3 are the most important readouts for HIF1a signaling ${ }^{26-28}$ and our data show positivity of all three target genes in the majority of both BCC and TE. Positive VEGF-A staining patterns were found in the endothelial cells of blood vessels as well as in BCC/TE tumor cells. However, VEGF-A expression was not specifically tumor related, since some weak staining was also noted in the basal cell layer of epidermis adjacent to the tumors. These low and weak VEGF-A expression levels are in agreement with earlier studies in BCC and other benign and malignant skin tumors ${ }^{29,30}$ and might be explained by the low levels of mTORC1 expression found (see below), since recent evidence indicates that $\mathrm{mTORC} 1$ can drive VEGF-A expression. ${ }^{19}$ It also fits with the low metastatic behavior of $\mathrm{BCC}^{29}$. Accordingly, strong VEGF-A expression is observed in the more aggressive and potentially metastasizing squamous cell carcinoma and melanoma. ${ }^{29,31}$

Furthermore, we found expression of PHD2 in $63.3 \%$ of TE and $43.9 \%$ of BCC specimens, whereas expression of HIF1a itself was low or even undetectable. Several in vitro studies have shown that PHD2 is transiently upregulated in a HIF-dependent manner under normoxic as well as mild hypoxic conditions, which could suggest that HIF might induce an autoinhibitory effect on its own activity. ${ }^{32}$ Moreover, D'Angelo et al demonstrated that hypoxic upregulation of PHD2 acts as a feedback mechanism to stop hypoxic signaling in reoxygenated cells. ${ }^{33}$ Hence, we postulate that the lack of HIF1a in BCC and TE specimens can be explained by the presence of PHD2.

Activity of the mTORC1 pathway was assessed by use of the upstream regulator PAKT and downstream target pS6. PAKT was positive in $88.9 \%$ of BCC and $87.5 \%$ of TE, while $55 \%$ of BCC and $61.3 \%$ of TE specimens were positive for pS6, supporting the presence of active mTOR signaling. The positive stains for PAKT and pS6 found in our study are consistent with the known activity of $\mathrm{PI}(3) \mathrm{K} / \mathrm{AKT}$ signaling in BCC. ${ }^{34}$ In addition, strong expression of pAKT and pS6 has been observed in a variety of skin neoplasms including Bowen's disease, keratoacanthoma, squamous cell carcinoma and extramammary Paget's disease..$^{35,36}$ However, despite positive staining of pAKT and pS6, hardly any mTORC1 phosphorylation at Ser-2448 was detected in both tumor types. This observation is consistent with two other studies reporting weak positivity of mTOR (Ser-2448) in only $7 \cdot 7 \%$ of $\mathrm{BCC}^{37}$ and $36 \%$ positivity among 85 epidermal tumors other than BCC. ${ }^{36}$ Ser-2448 is the mTORC1phosphorylation site modified either directly by AKT or by the downstream target of mTORC1, p70S6 kinase, making it the most important marker for activation of mTOR. ${ }^{38}$ However, the upregulation of up- and downstream target genes of the mTORC1 signaling cascade do 
suggest activation of downstream mTORC1 signaling components downstream, which could be attributed to $\mathrm{PI}(3) \mathrm{K}$ - signaling. ${ }^{39}$ In addition, it is known that multiple feedback loops exist, for example S6 kinase can dampen growth factor receptor signaling to PI3K.40

Overall, the immunohistochemical analysis is consistent with activity of HIF1 and mTORC1 signaling in both BCC and TE, in addition to the known PI(3)K-AKT activity in BCC. ${ }^{34}$ We also showed that the number of HIF1a positive cells was significantly higher in BCC than in TE, which might be explained by the fact that BCC tumor nests are generally larger than the TE tumor nests and consequently could be more hypoxic. Nodular BCC tend to have more GLUT1 and PHD2 expression when compared to superficial and infiltrative BCC, which could similarly be due to them being more hypoxic. Advanced insights in molecular pathways active in cancer development have already resulted in the development of novel topical and systemic targeted therapies as a rational approach to the management of many (skin) cancers. ${ }^{41}$ Our results suggest that it might be of interest to further explore the contribution of HIF1 and mTORC1 signaling to BCC and TE growth. Deeper insights into such signaling pathways might eventually result in the identification of novel targets for treatment. Finally a better understanding of alterations in gene expression could be used to develop better histological diagnostics, since at this moment immunohistochemical analysis of HIF, mTORC1 and their target genes does not provide a reliable diagnostic tool for the discrimination of BCC and TE. 


\section{References}

1. Flohil SC, Seubring I, van Rossum MM, Coebergh JW, de Vries E, Nijsten T. Trends in Basal cell carcinoma incidence rates: a 37-year Dutch observational study. The Journal of investigative dermatology 2013;133:913-8.

2. Rippey JJ. Why classify basal cell carcinomas? Histopathology 1998;32:393-8.

3. Youssef KK, Van Keymeulen A, Lapouge G, et al. Identification of the cell lineage at the origin of basal cell carcinoma. Nature cell biology 2010;12:299-305.

4. Kasper M, Jaks V, Hohl D, Toftgard R. Basal cell carcinoma - molecular biology and potential new therapies. The Journal of clinical investigation 2012;122:455-63.

5. Arits AH, Van Marion AM, Lohman BG, et al. Differentiation between basal cell carcinoma and trichoepithelioma by immunohistochemical staining of the androgen receptor: an overview. European journal of dermatology : EJD 2011;21:870-3.

6. Weedon D. Skin Pathology. 3rd ed. Tokyo: Churchill Livingstone; 2009.

7. Yang Y, Sun M, Wang L, Jiao B. HIFs, angiogenesis, and cancer. Journal of cellular biochemistry 2013;114:967-74.

8. Nys K, Maes H, Dudek AM, Agostinis P. Uncovering the role of hypoxia inducible factor-1alpha in skin carcinogenesis. Biochimica et biophysica acta 2011;1816:1-12.

9. Evans SM, Schrlau AE, Chalian AA, Zhang P, Koch CJ. Oxygen levels in normal and previously irradiated human skin as assessed by EF5 binding. The Journal of investigative dermatology 2006;126:2596-606.

10. van Steensel MA, van Geel M, Badeloe S, Poblete-Gutierrez P, Frank J. Molecular pathways involved in hair follicle tumor formation: all about mammalian target of rapamycin? Experimental dermatology 2009;18:18591.

11. Ruan K, Song G, Ouyang G. Role of hypoxia in the hallmarks of human cancer. Journal of cellular biochemistry 2009; 107:1053-62.

12. Chan DA, Giaccia AJ. PHD2 in tumour angiogenesis. British journal of cancer 2010;103:1-5.

13. Vasudev NS, Reynolds AR. Anti-angiogenic therapy for cancer: current progress, unresolved questions and future directions. Angiogenesis 2014;17:471-94.

14. Sadlecki P, Bodnar M, Grabiec M, et al. The role of Hypoxia-inducible factor-1 alpha, glucose transporter-1, (GLUT-1) and carbon anhydrase IX in endometrial cancer patients. BioMed research international 2014;2014:616850.

15. Warburg O. On the origin of cancer cells. Science 1956;123:309-14.

16. Sedlakova O, Svastova E, Takacova M, Kopacek J, Pastorek J, Pastorekova S. Carbonic anhydrase IX, a hypoxia-induced catalytic component of the $\mathrm{pH}$ regulating machinery in tumors. Frontiers in physiology 2014;4:400.

17. Chinnadurai G, Vijayalingam S, Gibson SB. BNIP3 subfamily BH3-only proteins: mitochondrial stress sensors in normal and pathological functions. Oncogene 2008;27 Suppl 1:S114-27.

18. Semenza GL. Defining the role of hypoxia-inducible factor 1 in cancer biology and therapeutics. Oncogene 2010;29:625-34.

19. Dodd KM, Yang J, Shen MH, Sampson JR, Tee AR. mTORC1 drives HIF-1alpha and VEGF-A signaling via multiple mechanisms involving 4E-BP1, S6K1 and STAT3. Oncogene 2014.

20. Arsham AM, Howell JJ, Simon MC. A novel hypoxia-inducible factor-independent hypoxic response regulating mammalian target of rapamycin and its targets. The Journal of biological chemistry 2003;278:29655-60.

21. Duvel K, Yecies JL, Menon S, et al. Activation of a metabolic gene regulatory network downstream of mTOR complex 1. Molecular cell 2010;39:171-83.

22. Brugarolas J, Lei K, Hurley RL, et al. Regulation of mTOR function in response to hypoxia by REDD1 and the TSC1/TSC2 tumor suppressor complex. Genes \& development 2004;18:2893-904. 
23. Wouters BG, Koritzinsky M. Hypoxia signaling through mTOR and the unfolded protein response in cancer. Nature reviews Cancer 2008;8:851-64.

24. Rathman-Josserand M, Genty G, Lecardonnel J, et al. Human Hair Follicle Stem/Progenitor Cells Express Hypoxia Markers. J Invest Dermatol 2013.

25. Krahl D, Sellheyer K. p75 Neurotrophin receptor differentiates between morphoeic basal cell carcinoma and desmoplastic trichoepithelioma: insights into the histogenesis of adnexal tumours based on embryology and hair follicle biology. The British journal of dermatology 2010;163:138-45.

26. Wykoff CC, Beasley NJ, Watson $\mathrm{PH}$, et al. Hypoxia-inducible expression of tumor-associated carbonic anhydrases. Cancer research 2000;60:7075-83.

27. Nakayama K. Cellular signal transduction of the hypoxia response. Journal of biochemistry 2009;146:75765.

28. Zhang HM, Cheung P, Yanagawa B, McManus BM, Yang DC. BNips: a group of pro-apoptotic proteins in the Bcl-2 family. Apoptosis : an international journal on programmed cell death 2003;8:229-36.

29. Bowden J, Brennan PA, Umar T, Cronin A. Expression of vascular endothelial growth factor in basal cell carcinoma and cutaneous squamous cell carcinoma of the head and neck. Journal of cutaneous pathology 2002;29:585-9.

30. Weninger W, Uthman A, Pammer J, et al. Vascular endothelial growth factor production in normal epidermis and in benign and malignant epithelial skin tumors. Laboratory investigation; a journal of technical methods and pathology 1996;75:647-57.

31. Toth-Jakatics R, Jimi S, Takebayashi S, Kawamoto N. Cutaneous malignant melanoma: correlation between neovascularization and peritumor accumulation of mast cells overexpressing vascular endothelial growth factor. Human pathology 2000;31:955-60.

32. Jokilehto T, Rantanen K, Luukkaa M, et al. Overexpression and nuclear translocation of hypoxia-inducible factor prolyl hydroxylase PHD2 in head and neck squamous cell carcinoma is associated with tumor aggressiveness. Clinical cancer research : an official journal of the American Association for Cancer Research 2006;12:1080-7.

33. D'Angelo G, Duplan E, Boyer N, Vigne P, Frelin C. Hypoxia up-regulates prolyl hydroxylase activity: a feedback mechanism that limits HIF-1 responses during reoxygenation. The Journal of biological chemistry 2003;278:38183-7.

34. Jee SH, Chiu HC, Tsai TF, et al. The phosphotidyl inositol 3-kinase/Akt signal pathway is involved in interleukin-6-mediated Mcl-1 upregulation and anti-apoptosis activity in basal cell carcinoma cells. The Journal of investigative dermatology 2002;119:1121-7.

35. Chen S, Nakahara T, Uchi H, et al. Immunohistochemical analysis of the mammalian target of rapamycin signaling pathway in extramammary Paget's disease. The British journal of dermatology 2009;161:357-63.

36. Chen SJ, Nakahara T, Takahara M, et al. Activation of the mammalian target of rapamycin signaling pathway in epidermal tumours and its correlation with cyclin-dependent kinase 2. The British journal of dermatology 2009;160:442-5.

37. Karayannopoulou G, Euvrard S, Kanitakis J. Differential expression of p-mTOR in cutaneous basal and squamous cell carcinomas likely explains their different response to mTOR inhibitors in organ-transplant recipients. Anticancer research 2013;33:3711-4.

38. Chiang GG, Abraham RT. Phosphorylation of mammalian target of rapamycin (mTOR) at Ser-2448 is mediated by p70S6 kinase. The Journal of biological chemistry 2005;280:25485-90.

39. Hardie DG. New roles for the LKB1-->AMPK pathway. Current opinion in cell biology 2005;17:167-73.

40. Fruman DA, Rommel C. PI3K and cancer: lessons, challenges and opportunities. Nature reviews 2014;13:140-56.

41. Liu LS, Colegio OR. Molecularly targeted therapies for nonmelanoma skin cancers. International journal of dermatology 2013;52:654-65. 


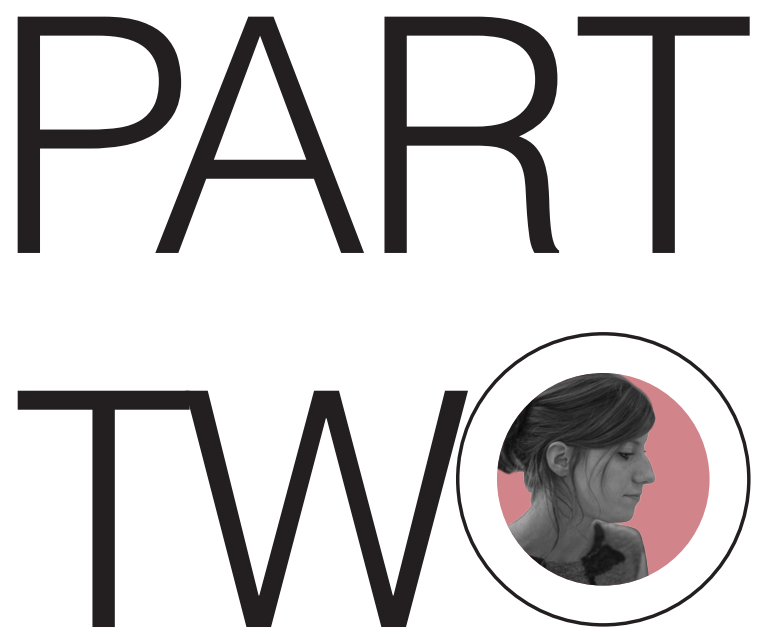

NEW TOPICAL AND SYSTEMIC TREATMENT MODALITIES FOR BASAL CELL CARCINOMA 


\section{Chapter 4.1}

The effect of topical diclofenac 3\% and calcitriol $3 \mu \mathrm{g} / \mathrm{g}$ on superficial basal cell carcinoma (sBCC) and nodular basal cell carcinoma (nBCC): A phase II, randomized controlled trial

Tjinta Brinkhuizen, Kiki JA. Frenckent, Patty J. Nelemans, Marlou LS. Hoff, Nicole WJ. Kelleners-Smeets, Axel zur Hausen, Michiel PJ. van der Horst, Dorit Rennspiess, Véronique JL. Winnepenninckx, Maurice AM. van Steensel, Klara Mosterd. 


\section{Abstract}

Background Non-steroidal anti-inflammatory drugs and vitamin D derivatives can target signaling pathways activated in Basal Cell Carcinoma (BCC).

Objective We investigated the efficacy of topically applied diclofenac sodium $3 \%$ gel, calcitriol $3 \mu \mathrm{g} / \mathrm{g}$ ointment and a combination of both in superficial (sBCC) and nodular (nBCC).

Methods Patients with a primary, histologically proven sBCC $(n=64)$ or nBCC $(n=64)$ were randomized to topical diclofenac, calcitriol, combination of both or no topical treatment (control group). After self-application twice daily under occlusion (8 weeks), tumors were excised. Primary outcome: post-treatment expression levels of proliferation (Ki67) and anti-apoptosis (Bcl-2) immunohistochemical markers. Secondary outcomes: histological clearance, adverse events, application-site reactions, patient compliance.

Results sBCCs treated with diclofenac showed a significant decrease in Ki-67 $(p<0.001)$ and Bcl-2 ( $p=0.001)$, and after combination therapy for Ki-67 ( $p=0.012)$. Complete histological tumor regression was seen in $64.3 \%(P=0.0003)$ of sBCCs (diclofenac) and $43.8 \%$ $(\mathrm{P}=0.007$ ) of sBCCs (combination therapy) compared to $0.0 \%$ of controls. No considerable changes were found in $\mathrm{nBCCs}$. Application-site reactions were mostly mild to moderate.

Limitations The small sample size.

Conclusion Our results suggest that topical diclofenac is a promising new treatment for SBCC. Its mode of action differs from available non-invasive therapies, and thus has an additive value.

\section{Capsule summary}

- $\quad$ Non-steroidal anti-inflammatory drugs and vitamin D derivatives can specifically target SHH and/or WNT signaling, activated in BCCs.

- $\quad$ Eight-weeks of topical diclofenac significantly reduced expression of proliferation and anti-apoptosis immunohistochemical markers and resulted in complete tumor regression in nearly two-thirds of treated sBCCs.

- $\quad$ Topical diclofenac is a promising non-invasive treatment for sBCCs. 


\section{Introduction}

Nonmelanoma skin cancer (NMSC) is the most common cancer among Caucasians. Sporadic basal cell carcinoma (BCC) accounts for $80 \%$ of all NMSCs and its incidence is still rising by $3-10 \%$ annually. ${ }^{1}$ The most common histological subtypes are nodular (nBCC) (40\%) and superficial BCC (sBCC) (18-31\%), both generally considered to be low-risk tumors. ${ }^{2}$ Conventional surgical excision is the gold standard in treatment of all BCCs, with success rates of $95-98 \% .^{3,4}$ However, for low-risk sBCCs, non-invasive treatment modalities such as photodynamic therapy (PDT), imiquimod (immune-modulating) and 5-fluorouracil cream (chemotherapeutic) are frequently prescribed. ${ }^{5}$ Tumor-free survival rates in SBCC vary from 72.8-84.0\% (PDT), 83.4$87.3 \%$ (imiquimod) and $80.1 \%$ (5-flourouracil). 5,6 Non-invasive treatments have several advantages, such as lower healthcare costs and a decrease in the workload for dermatologists. Also, a better cosmetic outcome with an increase in quality of life for patients can be expected. ${ }^{4}$

Current research on BCC focuses on treatments that specifically target key signaling pathways required for tumor growth. The Sonic Hedgehog $(\mathrm{SHH})$ signaling pathway is involved in the pathogenesis of essentially all sporadic $\mathrm{BCCs}^{7}$ and murine models show that canonical Wingless (WNT) signaling is essential for the tumorigenic response to deregulated $\mathrm{SHH}$ signaling in the skin. ${ }^{8}$ Interestingly, a recent study suggests that imiquimod, in addition to its immunomodulating effects, may actually serve as a targeted therapy by directly inhibiting $\mathrm{SHH}$ signaling. ${ }^{9}$ As shown in figure 1 , both $\mathrm{SHH}$ and WNT pathways can either directly or indirectly serve as therapeutic targets for non-steroidal anti-inflammatory drugs (NSAIDs) and the active form of vitamin $\mathrm{D}$ (calcitriol $\left(1 \mathrm{a}, 25[\mathrm{OH}]_{2} \mathrm{D} 3\right)$ ). NSAIDs were found to inhibit canonical WNT signaling in patients with familial adenomatous polyposis and are suggested to be pro-apoptotic in BCC cell lines in a cyclooxygenase-2 (COX-2) dependent and independent manner (fig 1). ${ }^{10-12}$ COX-2 is highly expressed in several solid tumors, including BCC. ${ }^{13}$ In a phase II clinical trial, systemic NSAIDs reduced both the number and burden of BCCs in patients with basal cell nevus syndrome. ${ }^{14}$ Additionally, topically applied diclofenac induced a clinical response in the majority of the patients with actinic keratosis, which can be a precursor of squamous cell carcinoma. ${ }^{15}$ The anti-tumor effects of calcitriol, as seen in model systems of several human malignancies such as cancer of the prostate, ovary and lungs, are mainly attributed to stimulation of the vitamin D receptor (VDR). ${ }^{16}$ In keratinocytes, the VDR has a regulatory role in $\mathrm{SHH}$ and WNT signaling by acting as a tumor suppressor, resulting in reduction of proliferation and differentiation and induction of apoptosis (programmed cell death) (fig 1). ${ }^{16}$

We sought to investigate the efficacy of topical application of either a NSAID or a vitamin D analogue on low-risk BCCs, by evaluating the effects on proliferation and apoptosis. Both are well-accepted drugs already available for treatment of diseases other than cancer, like muscle pain (NSAIDs) and psoriasis (calcitriol). ${ }^{10}$ We also investigated the effect of combination of both therapies, because we hypothesized that simultaneously targeting different signaling pathways or pathway elements may have a synergistic effect as suggested by several preclinical and clinical studies. ${ }^{17}$ 


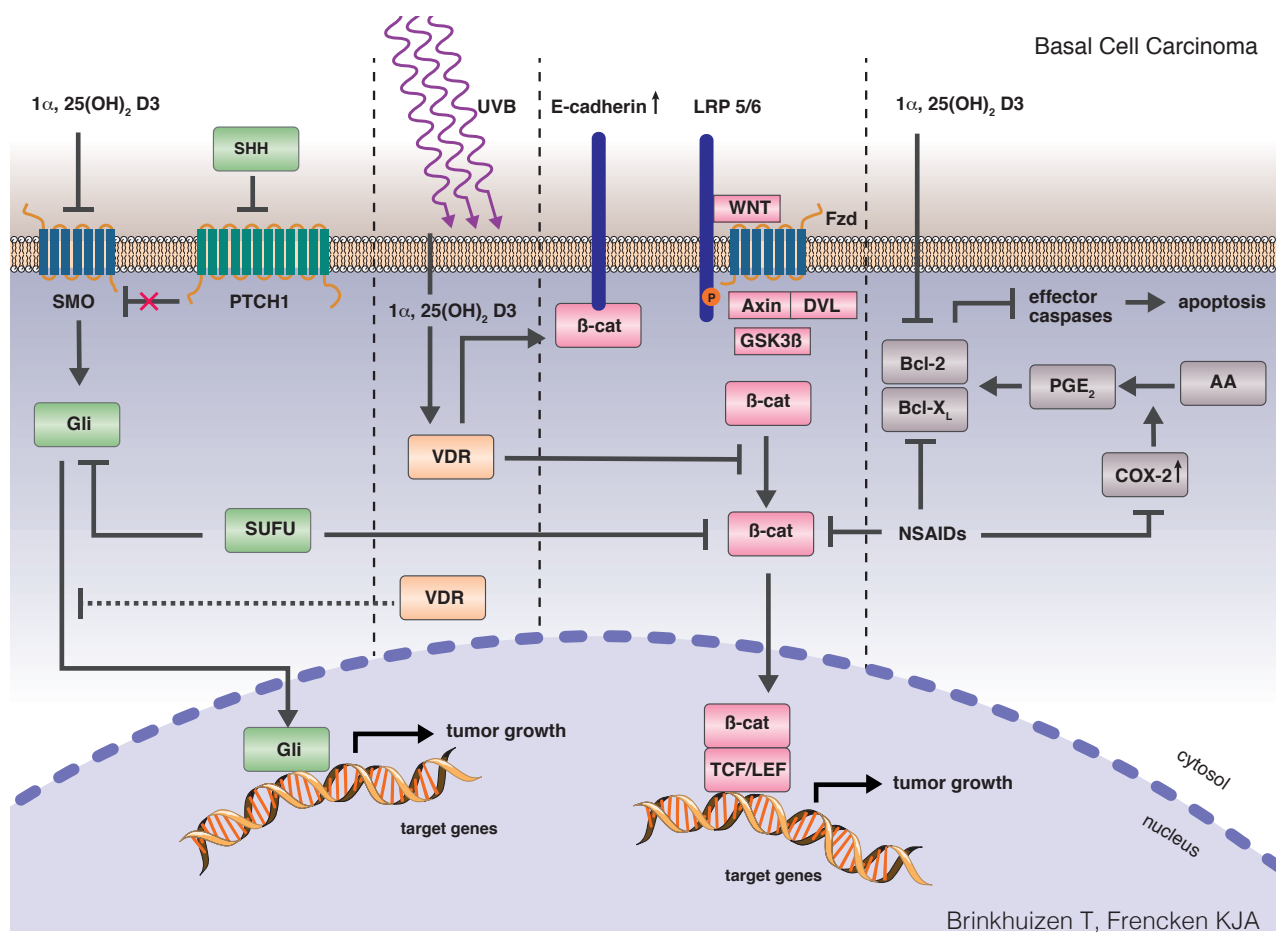

Figure 1. Actions of NSAIDs and Calcitriol in Basal Cell Carcinoma: a schematic overview.

Sonic Hedgehog Pathway (green). The extracellular protein Sonic hedgehog (SHH) binds to and inhibits Patched (PTCH1), a transmembrane receptor, which relieves the inhibition of another transmembrane protein, Smoothened (SMO). SMO activates glioma-associated oncogene homolog 1 (GLI1) and GLI2, transcription factors that travel into the nucleus to activate the expression of tumor-promoting genes.7 Canonical WNT signaling pathway (pink). Binding of a WNT ligand to its specific receptor complex containing a Frizzled (FZD) family member and LRP5 or LRP6 co-receptors, initiates WNT- $\beta$-catenin signaling. Axin relocates to the LRP $5 / 6$ tail at the membrane that is bound to WNT through its interaction with dishevelled (DVL), which forms a complex with GSK3 3 and prevents $\beta$-catenin ( $\beta$-cat) degradation. ${ }^{10}$ This allows $\beta$-catenin to accumulate and enter the nucleus, where it interacts with members of the TCF/LEF family. In the nucleus, $\beta$-catenin converts the TCF proteins into transcriptional activators. Suppressor of fused (SUFU) functions as a tumor suppressor by inhibiting both $\mathrm{SHH}$ and WNT signaling. ${ }^{8}$ NSAIDs inhibit WNT signaling by reducing nuclear $\beta$-catenin localization. ${ }^{10,31}$ Furthermore, NSAIDs inhibit cyclooxygenase-2 (COX-2), which is overexpressed in basal cell carcinoma (BCC) and catalyzes the conversion of arachidonic acid (AA) to prostaglandins (PGE2). A subsequent reduction of PGE2 and a direct down regulation of the anti-apoptotic $\mathrm{Bcl}-2$ family proteins by NSAIDs induces apoptosis. ${ }^{11} \mathrm{~A}$ down regulation of $\mathrm{Bcl}-2$ is also induced by $1 \mathrm{a}, 25(\mathrm{OH}) 2 \mathrm{D} 3$ (calcitriol), resulting in caspase cleavage leading to apoptosis. Calcitriol directly inhibits SMO in vitro, resulting in repression of $\mathrm{SHH}$ signaling. SHH-signaling is also suggested to be directly repressed by the Vitamin D Receptor (VDR) by inhibition of GLI. Finally, activation of the VDR by calcitriol induces the expression of the transmembrane protein E-Cadherin, which recruits $\beta$-catenin to the cell membrane and prevents translocation of $\beta$-catenin to the nucleus. ${ }^{16,32,33}$ 


\section{Methods}

\section{Protocol}

We performed a phase II, single-blind, randomized-controlled intervention trial. Patients from the dermatology outpatient clinic of the Maastricht University Medical Centre (MUMC), Maastricht, the Netherlands, were included between November $1^{\text {st }} 2011$ and February $15^{\text {th }} 2013$. Histologically proven primary sBCCs or (micro) nodular BCCs $\geq 4 \mathrm{~mm}$, not located in the face or on the hairy scalp, were eligible for inclusion and were asked to participate in the trial (appendix p 2). In case of mixed superficial and nodular BCC, tumors were categorized according to the most aggressive component (nBCC). Patients were excluded if they were using oral NSAIDs more than four days a week (chronic users) ${ }^{18}$ or vitamin D (containing) supplements in the preceding 30 days. The local medical ethics and scientific committee approved the protocol and two following amendments. The study was performed in accordance with the Declaration of Helsinki. All participants provided written informed consent.

Enrolled patients with a superficial BCC or nodular BCC were randomly assigned to receive either topical diclofenac sodium-3\% gel in hyaluronic acid 2.5\% (Solaraze ${ }^{\circledR}$, Almirall, Barcelona, Spain), calcitriol ointment $3 \mu \mathrm{g} / \mathrm{g}$ (Silkis ${ }^{\circledR}$, Galderma, Rotterdam, the Netherlands) (henceforward called diclofenac and calcitriol respectively), a combination of both (combination therapy), or no medication (control group).

The primary outcome measure was the post-treatment expression of $\mathrm{Ki}-67$ and $\mathrm{Bcl}-2$. Ki-67 is a proliferation marker, which can be detected in the nuclei of proliferating cells. Apoptosis is regulated by the proto-oncogene Bcl-2, which is overexpressed in the majority of the BCC. ${ }^{19} \mathrm{Immu}$ nohistochemical analysis is a reliable ap proach to evaluate treatment effects ${ }^{20,21}$ and was used to evaluate the percentage of cells expressing Ki-67 or Bcl-2. Secondary outcomes were histological tumor regression, adverse events, application-site reactions and patient compliance.

\section{Assignment and masking}

Randomizationwasstratifiedforhistological tumortypeand performedviaacomputer-generated randomallocation scheme. Random permuted blocks of eight were used to ensure concealment of allocation. Patients as well as investigators prescribing study treatment and collecting clinical data were not blinded to treatment assignment. The pathologists who assessed expression levels of $\mathrm{Ki}-67$ and $\mathrm{Bcl}-2$ and histological tumor regression were blinded to assigned treatment.

\section{Participant flow and follow-up}

Patients received the study medication from the investigator directly after randomization. The vehicle was self-applied on the tumor with a radius of $0.5 \mathrm{~cm}$ and covered with an occlusive sheet (Tegaderm $®$, 3M, Leiden, The Netherlands) twice a day for eight weeks. This period was generally the time patients waited for surgical excision. In case of combination therapy, diclofenac gel application was followed by calcitriol ointment with a two-minute interval. 
Treatment was continued until the day before surgery. In case of a severe local skin reaction, surgery was postponed. Surgical excision was performed with a 3-5mm safety margin. Expression of $\mathrm{Ki}-67$ and $\mathrm{Bcl}-2$ was evaluated in both baseline biopsies and excision specimens (appendix p2). No study related follow-up visits were planned after surgical excision.

Treatment reactions were evaluated by a phone interview two weeks after the start of the treatment. Furthermore, secondary outcome parameters were collected from a patient diary, which was filled-out once a week during the course of the treatment. The patient diary included questions on pain expressed on a visual analogue scale, local skin reactions and compliance. Standardized photographs of all lesions were taken with a ruler and pantone color card (Danes-Picta, Praha, Czech Republic) on day one and 56. Every consultation was documented. Compliance was defined as the number of actual applications as a percentage of the total prescribed number of applications.

\section{Statistical analysis}

In total, we aimed to include $64 \mathrm{nBCC}$ and 64 sBCC patients to enable comparison of the four study arms separately for sBCC and nBCC (appendix p2). Continuous variables were presented as a mean with \pm standard deviations (if normally distributed) or as a median with an interquartile range (if not normally distributed). Differences in proportions between groups were tested using the Fisher's exact test. Analysis of covariance (ANCOVA) was used to compare post-treatment expression of $\mathrm{Ki}-67$ and $\mathrm{Bcl}-2$ between treatment groups and control group. Variables indicating treatment group and baseline expression levels of $\mathrm{Ki}-67$ and $\mathrm{Bcl}-2$ were entered as independent variables. The regression coefficients associated with the treatment groups represent the difference in post-treatment expression level between the corresponding treatment group and the control group. In case of skewed baseline distributions of the of Ki-67 and $\mathrm{Bcl}-2$ expression levels data were log transformed to normalize distributions and normality of the distribution of residuals was checked using a normal probability plot. Statistical analyses were carried out using SPSS 20.0 software and www.openepi.com. All reported P values are two-sided, and $P$ values $\leq 0.05$ were considered statistically significant. This study is registered as a controlled trial at clinicaltrials.gov, number NCT01358045, since May $17^{\text {th }} 2011$. 


\section{Results}

\section{Patients}

One hundred and twenty-eight patients were included (64 nBCC and 64 sBCC) and randomly assigned to one of the four study arms (fig 2). The distribution of the baseline demographic and tumor characteristics was comparable between treatment groups (table 1). One patient withdrew directly after treatment allocation and thus information on all outcomes was missing. The primary outcome was not available for eight patients. One patient had a BCC that required treatment by Mohs' micrographic surgery and another patient had a syringoma. For six other patients, the biopsy or excision specimens did not include sufficient paraffin embedded tumor tissue to enable immunohistochemistry for $\mathrm{Ki}-67$ and $\mathrm{Bcl}-2$. The subjects with missing primary outcome were evenly distributed among the treatment groups. A total of 119 patients were included in the statistical analysis of the primary outcome ( $n=59$ sBCC and $n=60$ nBCC, fig 2). No crossovers occurred.

\section{Superficial BCC}

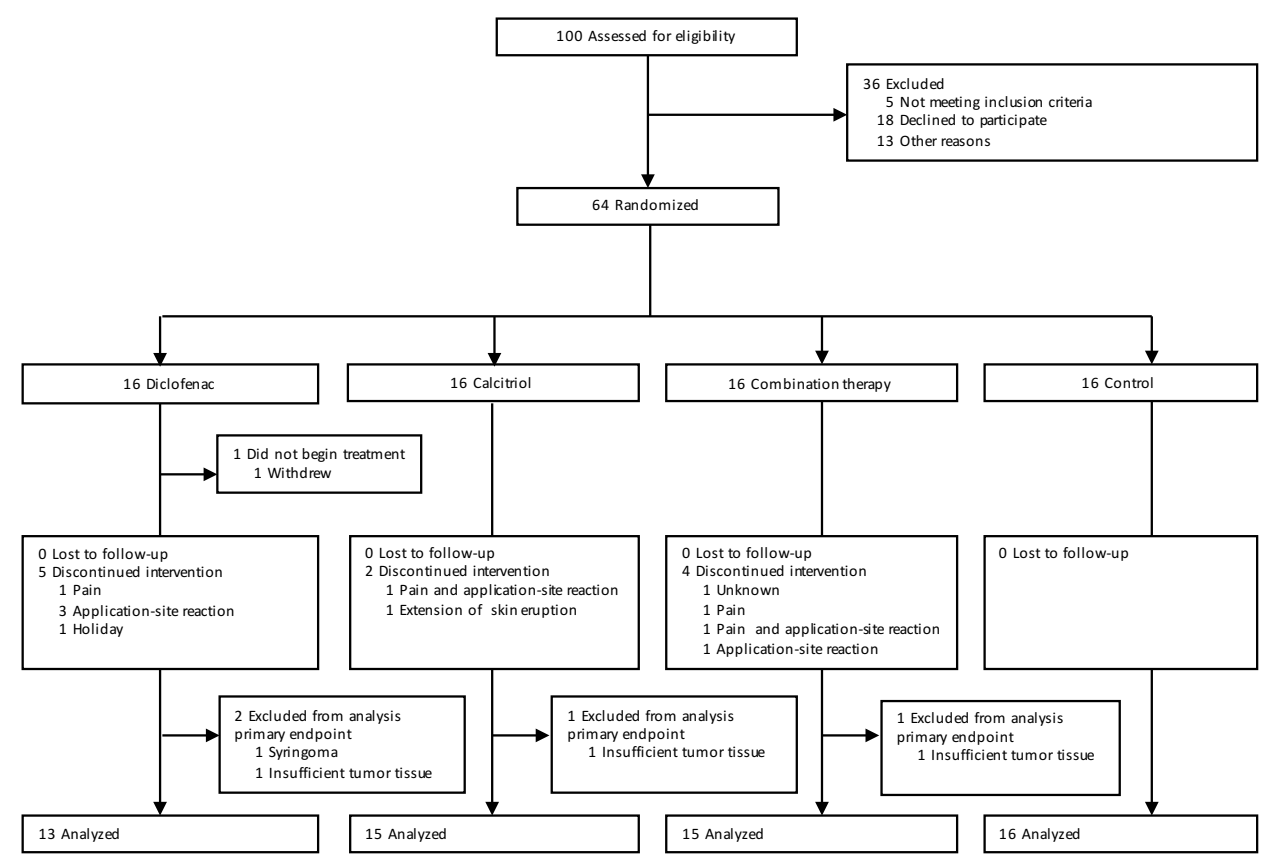

\section{Figure 2A: CONSORT diagram.}

Abbreviations: $\mathrm{BCC}=$ Basal Cell Carcinoma. Diclofenac=diclofenac sodium-3\% gel, Calcitriol=Calcitriol $3 \mu \mathrm{g} / \mathrm{g}$ ointment, Combination therapy=diclofenac and calcitriol. 


\section{Nodular BCC}

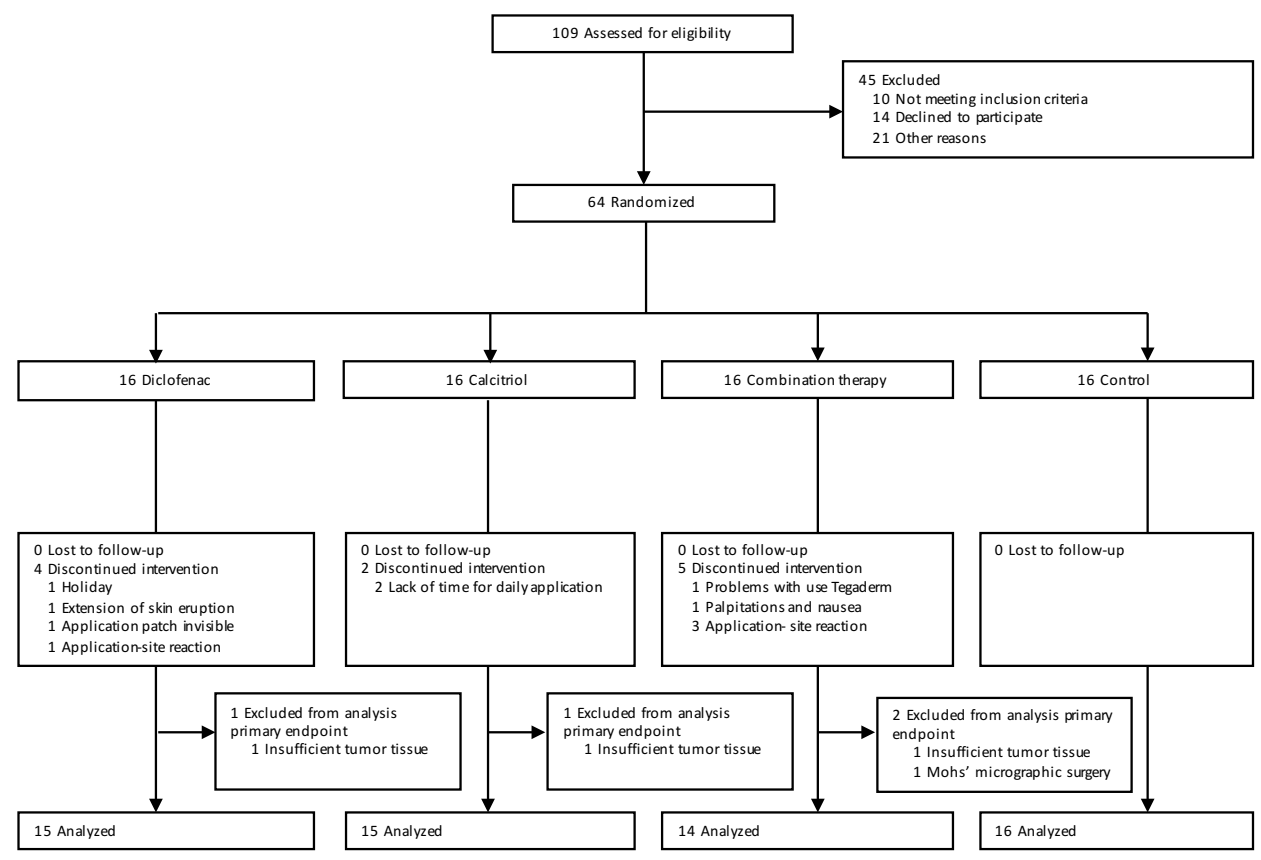

\section{Figure 2B: CONSORT diagram.}

Abbreviations: $\mathrm{BCC}=$ Basal Cell Carcinoma. Diclofenac=diclofenac sodium-3\% gel, Calcitriol=Calcitriol $3 \mu \mathrm{g} / \mathrm{g}$ ointment, Combination therapy=diclofenac and calcitriol.

\begin{tabular}{|c|c|c|c|c|}
\hline & $\begin{array}{r}\text { Diclofenac } \\
\qquad(n=16)\end{array}$ & $\begin{array}{r}\text { Calcitriol } \\
\qquad(n=16)\end{array}$ & $\begin{array}{l}\text { Combination therapy } \\
\qquad(n=16)\end{array}$ & $\begin{array}{r}\text { Control } \\
(n=16)\end{array}$ \\
\hline \multicolumn{5}{|l|}{ Superficial BCC } \\
\hline \multicolumn{5}{|l|}{ Sex } \\
\hline Male & $12(75 \%)$ & $13(81 \%)$ & $9(56 \%)$ & $11(69 \%)$ \\
\hline Female & $4(25 \%)$ & $3(19 \%)$ & $7(44 \%)$ & $5(31 \%)$ \\
\hline Age in years & $63.0(54-68)$ & $65.5(55-72)$ & $67.5(60-74)$ & $61.5(49-68)$ \\
\hline \multicolumn{5}{|l|}{ Localization BCC } \\
\hline Neck & $2(13 \%)$ & 0 & 0 & 0 \\
\hline Upper Extremity & $5(31 \%)$ & $8(50 \%)$ & $5(31 \%)$ & $6(38 \%)$ \\
\hline Trunk & $6(38 \%)$ & $8(50 \%)$ & $10(63 \%)$ & $5(31 \%)$ \\
\hline Lower Extremity & $3(19 \%)$ & 0 & $1(6 \%)$ & $5(31 \%)$ \\
\hline Other & 0 & 0 & 0 & 0 \\
\hline
\end{tabular}

Histology biopsy

Superficial BCC 


\begin{tabular}{|c|c|c|c|c|}
\hline $\begin{array}{l}\text { Tumor surface in } \\
\mathrm{mm}^{2}\end{array}$ & $61.7(30.0-84.4)$ & $54.2(44.8-87.6)$ & $46.7(37.1-101.3)$ & $59.7(43.6-74.2)$ \\
\hline $\begin{array}{l}\text { Percentage Ki-67 } \\
\text { expression }\end{array}$ & $20.0(6-37)$ & $5.5(4-32)$ & $21.0(12-49)$ & $10.0(6-35)$ \\
\hline $\begin{array}{l}\text { Percentage } \mathrm{Bcl}-2 \\
\text { expression }\end{array}$ & $8.5(4-52)$ & $10.0(4-63)$ & $19.0(5-65)$ & $5.0(1-25)$ \\
\hline \multicolumn{5}{|l|}{ Nodular BCC } \\
\hline \multicolumn{5}{|l|}{ Sex } \\
\hline Male & $12(75 \%)$ & $12(75 \%)$ & $11(69 \%)$ & $9(56 \%)$ \\
\hline Female & $4(25 \%)$ & $4(25 \%)$ & $5(31 \%)$ & $7(44 \%)$ \\
\hline Age in years & $78.5(62-82)$ & $68.5(61-75)$ & $71(68-79)$ & $66(56-73)$ \\
\hline \multicolumn{5}{|l|}{ Localization BCC } \\
\hline Neck & 0 & $1(6 \%)$ & $2(13 \%)$ & $1(6 \%)$ \\
\hline Upper Extremity & $1(6 \%)$ & $3(19 \%)$ & $6(38 \%)$ & $3(19 \%)$ \\
\hline Trunk & $9(56 \%)$ & $10(63 \%)$ & $4(25 \%)$ & $9(56 \%)$ \\
\hline Lower Extremity & $6(38 \%)$ & $2(13 \%)$ & $3(19 \%)$ & $3(19 \%)$ \\
\hline Other & 0 & 0 & $1(6 \%)$ & 0 \\
\hline \multicolumn{5}{|l|}{ Histology biopsy } \\
\hline Nodular BCC & $16(100 \%)$ & $13(81 \%)$ & 15 (94\%) & $14(88 \%)$ \\
\hline Micro nodular BCC & 0 & $1(6 \%)$ & 0 & $1(6 \%)$ \\
\hline Mixed type & 0 & $2(13 \%)$ & 0 & $1(6 \%)$ \\
\hline $\begin{array}{l}\text { Tumor surface in } \\
\mathrm{mm}^{2}\end{array}$ & 49.5 (31.4-70.7) & $59.7(34.3-87.6)$ & $44.8(33.0-53.8)$ & $53.4(39.1-98.4)$ \\
\hline $\begin{array}{l}\text { Percentage Ki-67 } \\
\text { expression }\end{array}$ & $10.0(5-13)$ & $14.5(6-21)$ & $10.5(9-28)$ & $8.5(6-14)$ \\
\hline $\begin{array}{l}\text { Percentage } \mathrm{Bcl}-2 \\
\text { expression }\end{array}$ & $22.0(4-69)$ & $47.5(6-80)$ & $28.5(5-76)$ & $42.5(8-80)$ \\
\hline \multicolumn{5}{|c|}{$\begin{array}{l}\text { Continuous variables are expressed as median (interquartile range) and categorical variables as n (\%). } \\
\text { Abbreviation: } \mathrm{BCC}=\text { Basal Cell Carcinoma. Diclofenac=diclofenac sodium- } 3 \% \text { gel, Calcitriol=Calcitriol } 3 \mu \mathrm{g} / \mathrm{g} \\
\text { ointment, Combination therapy=diclofenac and calcitriol. * Percentages have been rounded and therefore do not } \\
\text { always total } 100 .\end{array}$} \\
\hline
\end{tabular}

\section{Immunohistochemical analysis of proliferation and apoptosis}

Figure 3 illustrates the median expression levels of Ki-67 and Bcl-2 in tumor cells before and after treatment for $\mathrm{sBCC}$ and $\mathrm{nBCC}$ in the four randomized groups. With respect to sBCCs, this figure shows that in the control group median values of endpoint Ki-67 expression levels increased slightly when compared with baseline levels. There was a substantial decrease in Ki-67 expression after treatment with diclofenac and combination therapy and a small increase in the calcitriol group. Median values in $\mathrm{Bcl}-2$ expression show a small increase in the control group, whereas there was a slight decrease in tumors treated with diclofenac, calcitriol and combination treatment. In the appendix photomicrographs of the changes on the immunohistochemical stains were given (appendix pp 3 and 4). 
superficial bcc

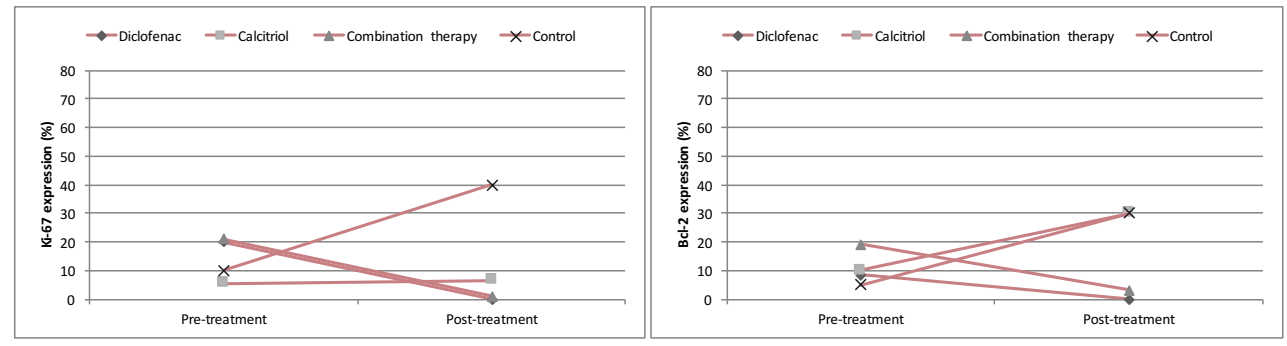

nodular bcc

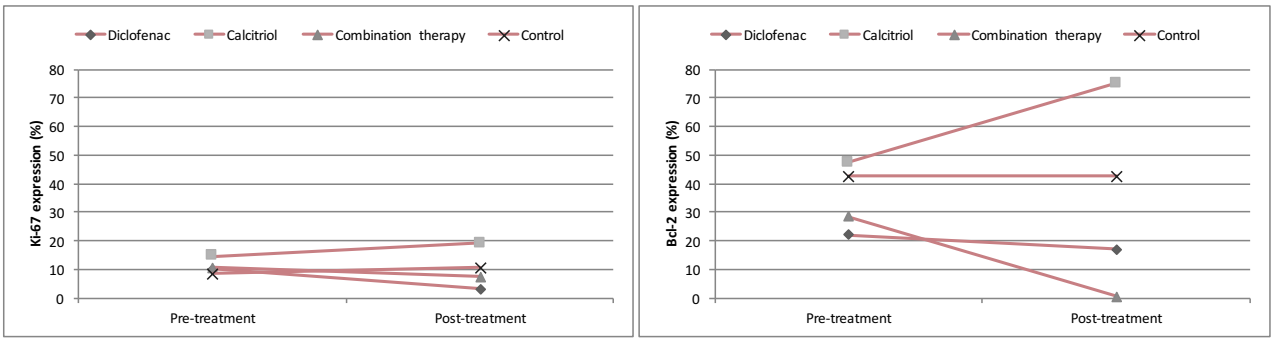

Figure 3: Pre- versus post-treatment scatterplot of changes in $\mathrm{Ki}-67$ and $\mathrm{Bcl}-2$ expression.

Data are median \%.Abbreviation: BCC=Basal Cell Carcinoma. Diclofenac=diclofenac sodium-3\% gel, Calcitriol=calcitriol $3 \mu \mathrm{g} / \mathrm{g}$ ointment, Combination therapy=diclofenac and calcitriol. Pre- versus post-treatment changes in $\mathrm{Ki}-67$ and $\mathrm{Bcl}-2$ expression after 8 weeks treatment according to treatment groups.

The distributions of $\mathrm{Ki}-67$ and $\mathrm{Bcl}-2$ expression levels were skewed and a $\log (\mathrm{x}+1)$ transformation was used to normalize the distributions. ANCOVA analyses were used to adjust for imbalances in baseline values of $\mathrm{Ki}-67$ and $\mathrm{Bcl}-2$. The mean differences between the treatment groups and the control group were back transformed from the logarithm scale to raw scale and are presented in Table 2. Ki-67 expression was significantly lower in SBCCs treated with diclofenac and combination therapy, when compared to the post treatment levels in the control group $(p<0.001$ and $p=0.012$, respectively, table 2). Also, Bcl-2 expression was significantly lower in sBCCs treated with diclofenac $(p=0.001$, table 2). Post treatment expression levels in sBCCs treated with calcitriol did not differ significantly from those in the control group. With respect to nBCCs, there were no statistically significant differences in expression levels in excision specimens, neither for Ki-67 nor for Bcl-2, between treatment groups and the control group (Table 2). 
Table 2: Mean difference in post treatment percentage of cells with expression of Ki-67 or Bcl-2 between treatment and control group, after adjustment for baseline differences using ANCOVA

\begin{tabular}{|c|c|c|c|c|c|c|}
\hline & $\begin{array}{l}\text { Mean } \\
\text { erence }\end{array}$ & $95 \% \mathrm{Cl}$ & $P$ & $\begin{array}{l}\text { Mean } \\
\text { erence }\end{array}$ & $95 \% \mathrm{Cl}$ & $\mathrm{P}$ \\
\hline \multicolumn{7}{|c|}{$\begin{array}{l}\text { Superficial BCC } \\
(n=59)\end{array}$} \\
\hline Diclofenac & -6.37 & -19.01 to -1.72 & $<0.001$ & -6.63 & -21.67 to -1.57 & 0.001 \\
\hline Calcitriol & -0.70 & -2.77 to 0.30 & 0.179 & -0.45 & -3.33 to 1.07 & 0.492 \\
\hline $\begin{array}{l}\text { Combination } \\
\text { therapy }\end{array}$ & -3.61 & -13.81 to -0.44 & 0.012 & -0.97 & -8.14 to 1.35 & 0.372 \\
\hline \multicolumn{7}{|l|}{$\begin{array}{l}\text { Nodular BCC } \\
(n=60)\end{array}$} \\
\hline Diclofenac & -0.59 & -2.55 to 0.40 & 0.246 & -0.59 & -3.50 to 0.78 & 0.367 \\
\hline Calcitriol & +0.39 & -0.54 to 1.97 & 0.386 & +0.82 & -0.71 to 4.71 & 0.290 \\
\hline $\begin{array}{l}\text { Combination } \\
\text { therapy }\end{array}$ & -0.60 & -3.12 to 0.62 & 0.321 & -1.12 & -5.72 to 0.50 & 0.193 \\
\hline \multicolumn{7}{|c|}{$\begin{array}{l}\text { Data represent mean difference in post treatment percentage of cells with expression between treatment and } \\
\text { control group, after adjustment for baseline differences. P-values are calculated with ANCOVA. } \\
\text { Abbreviation: BCC=Basal cell carcinoma. Diclofenac=diclofenac sodium-3\% gel, Calcitriol=calcitriol } 3 \mu \mathrm{g} / \mathrm{g} \text { oint- } \\
\text { ment, Combination therapy=diclofenac and calcitriol. }\end{array}$} \\
\hline
\end{tabular}

\section{Clinical response and compliance}

In the sBCC subgroup, histologically complete tumor regression was seen in $64.3 \%$ (9 of 14 ) and in $43.8 \%$ (7 of 16) of the tumors treated with diclofenac and combination therapy respectively (fig 4). The difference with the control group (0 of 16) was statistically significant $(P=0.0003$ and $P=0.007$, respectively). None of the participants in the calcitriol group showed complete regression. In the nBCC subgroup $31.2 \%$ (5 of 16), $6.2 \%$ (1 of 16), 33.3\% (5 of 15) showed histological complete regression in patients treated with diclofenac, calcitriol or combination therapy, respectively. No residual tumor was observed in 18.8\% (3 of 16) of the nBCC control group (fig 4). Differences between active treatment groups and the control group were not statistically significant. Evaluation of excision specimens of the SBCC subgroup with no complete tumor regression revealed a nodular BCC component in four tumors assigned to the combination therapy group. In the calcitriol group two tumors with a nodular component and one tumor with an invasive BCC were found. Data on compliance were available for 95 patients. Median compliance rates were generally high (92.7\%-98.2\%) and comparable between groups (appendix p 5). 
superficial bcc
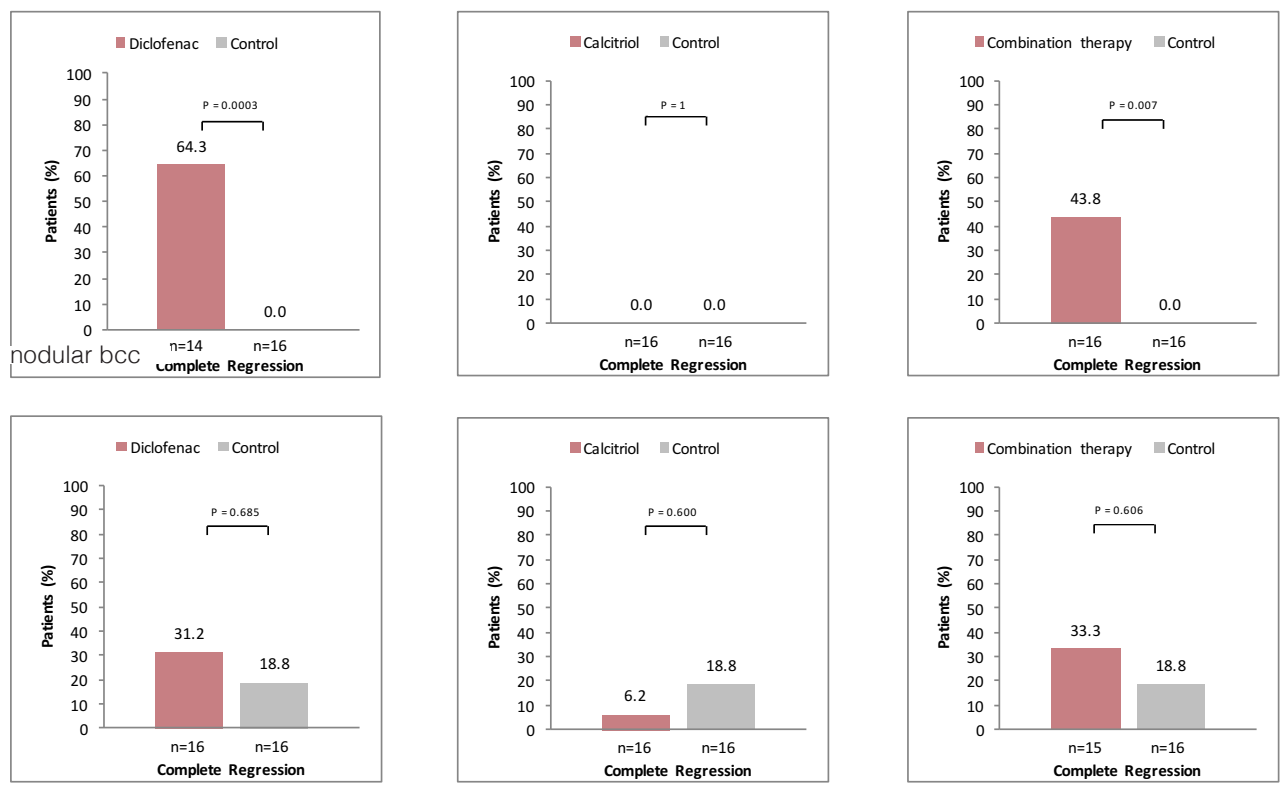

Figure 4: Complete histologic tumor regression rates after 8 weeks of topical treatment.

Abbreviation: $\mathrm{BCC}=$ Basal Cell Carcinoma. Diclofenac=diclofenac sodium-3\% gel, Calcitriol=calcitriol 3 $\mu \mathrm{g} / \mathrm{g}$ ointment, Combination therapy=diclofenac and calcitriol. P-values were calculated with Fisher exact test. Complete histologic tumor regression for superficial BCC and for nodular BCC according to treatment groups, compared to control group.

\section{Adverse events}

Adverse events were comparable for sBCC and $\mathrm{nBCC}$, with mostly mild to moderate severity (appendix pp 7-10). Erythema, pruritus and erosions at the target tumor site were the most frequently reported application-site reactions (table 3). In eight cases the severity of the application-site reactions led to discontinuation of the therapy. A topical antimicrobial cream was prescribed in those cases. In 19.4\% (6 of 31) patients treated with diclofenac and $9.4 \%$ (3 of 32 ) patients treated with combination therapy, surgical excision had to be postponed two weeks, due to the severity of investigator-observed application-site reactions. Three patients had serious adverse events requiring treatment and were hospitalized (table 3), but none of these serious adverse events were considered to be related to the study medication (appendix p 9). No adverse events were reported in the control group. 
Table 3. Adverse events according to treatment groups*

$\begin{array}{rrrr}\text { Diclofenac } & \text { Calcitriol } & \text { Combination therapy } & \text { Control } \\ (\mathrm{N}=31) & (\mathrm{N}=32) & (\mathrm{N}=32) & (\mathrm{N}=32)\end{array}$

\section{Event}

Any adverse event

Serious adverse

event $\emptyset$

Adverse event lead-

ing to permanent

discontinuation

Pain at application

site \|

$28(90.3 \%)$

$2(6.5 \%)$

$8(25.8 \%)$

$12(38.7 \%)$

at application site ||

Application-site

reactions $\int$

$\begin{array}{lccc}\text { Erythema } & 21(67.7 \%) & 20(62.5 \%) & 22(68.8 \%) \\ \text { Swelling } & 12(38.7 \%) & 7(21.9 \%) & 10(31.2 \%) \\ \text { Wounds } & 12(38.7 \%) & 9(28.1 \%) & 13(40.6 \%) \\ \text { Crusts } & 11(35.5 \%) & 5(15.6 \%) & 10(31.2 \%) \\ \text { Vesicles } & 11(35.5 \%) & 7(21.9 \%) & 7(21.9 \%) \\ \text { Scaling } & 6(19.4 \%) & 3(9.4 \%) & 4(12.5 \%) \\ \text { Pruritus } & 16(51.6 \%) & 11(34.4 \%) & 14(43.8 \%) \\ \text { Paresthesia } & 5(16.1 \%) & 2(6.2 \%) & 5(15.6 \%)\end{array}$

Data are $n(\%)$. Diclofenac=diclofenac sodium $-3 \%$ gel, Calcitriol=Calcitriol $3 \mu \mathrm{g} / \mathrm{g}$ ointment, Combination therapy=diclofenac and calcitriol.

* The events included are those that occurred at the time the first dose of the study drug was administered or any time thereafter, up to 14 days after excision. ๆ Serious adverse events where as follows: hospitalization due to fever and hypotension probably associated with a non-Hodgkin lymphoma, hospitalization due to fever associated with recurrent urinary tract infections after kidney transplantation, hospitalization due to fever associated with erysipelas, 3 days after excision. || Pain and burning sensation scores were assessed using a $10 \mathrm{~cm}$ Visual Analogue Scale (VAS) where 0 represented 'no pain' and 10 represented, 'most severe pain imaginable'. We computed variables into mild (VAS 1-3), moderate (VAS 4-6) and severe (VAS 7-10) (appendix p 6). J Application-site reactions were reported by the participants in a week diary and scored by mild, moderate or severe (appendix $\mathrm{p} 6$ ). 


\section{Discussion}

This phase II trial provides evidence that topical diclofenac has the potential to clear sBCC, with complete histologic tumor regression in $64.3 \%$ (9 of 14) and $43.8 \%$ (7 of 16) of the patients treated with diclofenac and combination therapy, respectively. These results are in line with significant decreases of expression levels of the proliferative marker Ki-67 and the anti-apoptotic marker $\mathrm{Bcl}-2$ in these treatment groups. Although, application-site reactions were reported frequently, most reactions were of mild to moderate severity, which is in accordance with the literature. ${ }^{22}$ Similar reactions are seen in other non-invasive therapies for sBCC, such as imiquimod and 5-fluorouracil cream. Those reactions are postulated to be necessary to achieve tumor regression and are indicators of a therapeutic effect. ${ }^{3,5}$ Repetitive occlusion may have attributed to the severity of the skin reactions.

Our results do not corroborate effectiveness of calcitriol. In calcitriol treated sBCCs, slight increases in expression levels of the proliferative marker Ki-67 and slight decreases in the anti-apoptotic marker $\mathrm{Bcl}-2$ between baseline levels and end point levels were detected, and differences in endpoint levels when compared with the control group were not statistically significant. We found no evidence for the hypothesized synergistic effect of the combination of diclofenac and calcitriol. The observed clinical effect of the combination therapy in SBCC is probably due to the effect of diclofenac. A lower diclofenac concentration following dilution by the calcitriol or the relatively high ratio of nodular BCC components in four tumors diagnosed as SBCC in the combination therapy group are possible explanations for clinical treatment failure. In more than 15\% of BCC of mixed histology the most aggressive histological subtype is missed by punch biopsy. ${ }^{23}$ An uneven distribution of misdiagnosed subtypes among treatment groups despite randomization is probably due to the relatively low sample size within treatment groups.

In the nBCC subgroup, we did not detect significant effects of treatment on Ki-67 and $\mathrm{Bcl}-2$ expression levels or in histological tumor clearance. However, when comparing post treatment expression levels of Ki-67 between the calcitriol and the control group (fig. 3), calcitriol treated nBCCs had slightly, although not significantly, higher $\mathrm{Ki}-67$ and $\mathrm{Bcl}-2$ expression. Previous in vitro studies found that high doses of calcitriol can inhibit keratinocyte proliferation, while lower doses may stimulate proliferation. ${ }^{24,25}$ As topical application of low dose calcitriol to mouse skin can even stimulate epidermal proliferation ${ }^{26}$, this might have been the case in the nBCCs treated with calcitriol.

An explanation for the lack of effect of calcitriol in general may be that the calcitriol ointment did not contain hyaluronic acid, whereas the diclofenac sodium-3\% gel contains $2.5 \%$ hyaluronic acid. Excipients such as hyaluronic acid may enhance the penetrance and bioavailability of a substance. ${ }^{27}$ It is possible that a higher concentration of calcitriol and/or a different vehicle might be needed to obtain clinical tumor regression. 
A complete tumor regression was seen in $18.8 \%$ of the controls in the nBCC subgroup. As we included tumors $\geq 4 \mathrm{~mm}$ and punch biopsies were $3 \mathrm{~mm}$, total tumor clearance could be a result of a previously reported biopsy-induced local immune response ${ }^{28}$, or a lack of sensitivity of regular histological techniques to detect small amounts of residual tumor. The presence of "therapeutic biopsy", found in the control group is an event that could have occurred in all other treatment groups and underlines the importance of a control group in clinical trials. The difference in response between $\mathrm{nBCC}$ and SBCC is in agreement with reports from other studies investigating non-invasive therapies and is presumably caused by insufficient penetration of the drug into the deeper dermis. ${ }^{29}$

In this study, the use of readily available diclofenac and calcitriol compounds was explored, without previous studies of dose-response effects for this indication. The clinical effect of diclofenac was higher than expected, but not as high as effects of currently available non-invasive BCC treatments. However, the expected major benefit of the investigated therapies is their ability to attack different molecular pathways activated in BCC. Evidence has already suggested that simultaneously targeting $\mathrm{SHH}$ and other signaling pathways may have a synergistic effect. ${ }^{17}$ Combination of therapies is therefore a logical next step in improving topical treatments. From this perspective, combining diclofenac with imiquimod cream could be promising. Imiquimod is known to inhibit the $\mathrm{SHH}$-pathway and is currently the most effective non-invasive therapy for BCC. However, with a 1-year efficacy of $83.4 \%{ }^{30}$, it is still not as effective as surgery, the gold standard. By adding a drug targeting a different pathway, such as diclofenac cream, the imiquimod resistant cells could be attacked, resulting in higher long-term cure rates.

Limitations of the study are the small sample size and imbalances in baseline levels of Ki-67 and Bcl-2 Despite the small sample size, significant effects of diclofenac treatment in targeting key signaling pathways could be demonstrated and ANCOVA was used to adjust for the differences in baseline levels.

This trial provides evidence that topical application of diclofenac 3\% gel in $2.5 \%$ hyaluronic acid in SBCC significantly reduces proliferation, induces apoptosis and moreover results in significant histological clearance compared to the control group. We therefore conclude that although surgical excision remains the gold standard for all BCC, topical diclofenac may be a promising new treatment for low risk sBCC. Efficacy of topical calcitriol was not observed. Other trials using different concentrations, excipients or combinations of both investigated drugs may be useful to optimize treatment strategies. Also, given the effectiveness of diclofenac gel in treatment of both BCCs and actinic keratosis with only limited side effects, it is tempting to speculate that there might be a role for topical diclofenac as a prophylactic agent in NMSC. This might be an interesting hypothesis to test in future studies. 


\section{References}

1. Lomas A, Leonardi-Bee J, Bath-Hextall F. A systematic review of worldwide incidence of nonmelanoma skin cancer. The British journal of dermatology 2012;166:1069-80.

2. Raasch BA, Buettner PG, Garbe C. Basal cell carcinoma: histological classification and body-site distribution. The British journal of dermatology 2006;155:401-7.

3. Telfer NR, Colver GB, Morton CA, British Association of D. Guidelines for the management of basal cell carcinoma. The British journal of dermatology 2008;159:35-48.

4. Bath-Hextall F, Ozolins M, Armstrong SJ, et al. Surgical excision versus imiquimod $5 \%$ cream for nodular and superficial basal-cell carcinoma (SINS): a multicentre, non-inferiority, randomised controlled trial. The lancet oncology 2014;15:96-105.

5. Arits $\mathrm{AH}$, Mosterd K, Essers BA, et al. Photodynamic therapy versus topical imiquimod versus topical fluorouracil for treatment of superficial basal-cell carcinoma: a single blind, non-inferiority, randomised controlled trial. The lancet oncology 2013;14:647-54.

6. Roozeboom MH, Arits AH, Nelemans PJ, Kelleners-Smeets NW. Overall treatment success after treatment of primary superficial basal cell carcinoma: a systematic review and meta-analysis of randomized and nonrandomized trials. The British journal of dermatology 2012;167:733-56.

7. Epstein EH. Basal cell carcinomas: attack of the hedgehog. Nature reviews Cancer 2008;8:743-54.

8. Yang SH, Andl T, Grachtchouk V, et al. Pathological responses to oncogenic Hedgehog signaling in skin are dependent on canonical Wnt/beta3-catenin signaling. Nature genetics 2008;40:1130-5.

9. Wolff F, Loipetzberger A, Gruber W, Esterbauer H, Aberger F, Frischauf AM. Imiquimod directly inhibits Hedgehog signalling by stimulating adenosine receptor/protein kinase A-mediated GLI phosphorylation. Oncogene 2013;32:5574-81.

10. Barker N, Clevers H. Mining the Wnt pathway for cancer therapeutics. Nature reviews 2006;5:997-1014.

11. Fecker LF, Stockfleth E, Nindl I, Ulrich C, Forschner T, Eberle J. The role of apoptosis in therapy and prophylaxis of epithelial tumours by nonsteroidal anti-inflammatory drugs (NSAIDs). The British journal of dermatology 2007;156 Suppl 3:25-33.

12. Tjiu JW, Liao YH, Lin SJ, et al. Cyclooxygenase-2 overexpression in human basal cell carcinoma cell line increases antiapoptosis, angiogenesis, and tumorigenesis. The Journal of investigative dermatology 2006;126:1143-51.

13. Muller-Decker K. Cyclooxygenase-dependent signaling is causally linked to non-melanoma skin carcinogenesis: pharmacological, genetic, and clinical evidence. Cancer metastasis reviews 2011;30:343-61.

14. Tang JY, Aszterbaum M, Athar M, et al. Basal cell carcinoma chemoprevention with nonsteroidal anti-inflammatory drugs in genetically predisposed PTCH1+/- humans and mice. Cancer Prev Res (Phila) 2010;3:25-34.

15. Criscione VD, Weinstock MA, Naylor MF, Luque C, Eide MJ, Bingham SF et al. Actinic keratoses: Natural history and risk of malignant transformation in the Veterans Affairs Topical Tretinoin Chemoprevention Trial. Cancer 2009; 115:2523-30.

16. Deeb KK, Trump DL, Johnson CS. Vitamin D signalling pathways in cancer: potential for anticancer therapeutics. Nature reviews Cancer 2007;7:684-700.

17. Brechbiel J, Miller-Moslin K, Adjei AA. Crosstalk between hedgehog and other signaling pathways as a basis for combination therapies in cancer. Cancer treatment reviews 2014.

18. Schnitzer TJ, Kong SX, Mavros PP, Straus WL, Watson DJ. Use of nonsteroidal anti-inflammatory drugs and gastroprotective agents before the advent of cyclooxygenase-2-selective inhibitors: analysis of a large United States claims database. Clin Ther 2001;23:1984-98.

19. Vidal D, Matias-Guiu X, Alomar A. Efficacy of imiquimod for the expression of Bcl-2, Ki-67, p53 and basal cell carcinoma apoptosis. The British journal of dermatology 2004;151:656-62. 
The effect of topical diclofenac and calcitriol on sBCC and sBCC: a phase II, randomized controlled trial | 129

20. Cho EA, Moloney FJ, Cai H, et al. Safety and tolerability of an intratumorally injected DNAzyme, Dz13, in patients with nodular basal-cell carcinoma: a phase 1 first-in-human trial (DISCOVER). Lancet 2013;381:1835-43.

21. Kim DJ, Kim J, Spaunhurst K, et al. Open-label, exploratory phase II trial of oral itraconazole for the treatment of basal cell carcinoma. Journal of clinical oncology : official journal of the American Society of Clinical Oncology 2014;32:745-51.

22. Ulrich C, Johannsen A, Rowert-Huber J, Ulrich M, Sterry W, Stockfleth E. Results of a randomized, placebo-controlled safety and efficacy study of topical diclofenac 3\% gel in organ transplant patients with multiple actinic keratoses. European journal of dermatology : EJD 2010;20:482-8.

23. Roozeboom MH, Mosterd K, Winnepenninckx VJ, Nelemans PJ, Kelleners-Smeets NW. Agreement between histological subtype on punch biopsy and surgical excision in primary basal cell carcinoma. Journal of the European Academy of Dermatology and Venereology : JEADV 2013;27:894-8.

24. Bikle DD, Oda Y, Xie Z. Vitamin D and skin cancer: a problem in gene regulation. The Journal of steroid biochemistry and molecular biology 2005;97:83-91.

25. Bollag WB, Ducote J, Harmon CS. Biphasic effect of 1,25-dihydroxyvitamin D3 on primary mouse epidermal keratinocyte proliferation. Journal of cellular physiology 1995;163:248-56.

26. Lutzow-Holm C, De Angelis P, Grosvik H, Clausen OP. 1,25-Dihydroxyvitamin D3 and the vitamin D analogue $\mathrm{KH} 1060$ induce hyperproliferation in normal mouse epidermis. A BrdUrd/DNA flow cytometric study. Experimental dermatology 1993;2:113-20.

27. Ulrich M, Stockfleth E. Field treatment of actinic keratoses - focus on COX-2-inhibitors. Actas dermo-sifiliograficas 2009;100 Suppl 2:55-8.

28. Swetter SM, Boldrick JC, Pierre P, Wong P, Egbert BM. Effects of biopsy-induced wound healing on residual basal cell and squamous cell carcinomas: rate of tumor regression in excisional specimens. Journal of cutaneous pathology 2003;30:139-46.

29. Rippey JJ. Why classify basal cell carcinomas? Histopathology 1998;32:393-8.

30. Arits AHMM, Mosterd K, Essers BAB, et al. Photodynamic therapy versus topical imiquimod versus topical fluorouracil for treatment of superficial basal-cell carcinoma: A single blind, non-inferiority, randomised controlled trial. The lancet oncology 2013;14:647-54. 


\section{Appendix}

\section{Eligibility criteria}

Patients with a histologically proven primary superficial BCC or (micro)nodular BCC with a diameter of at least $4 \mathrm{~mm}$, not located in the face or hairy scalp were eligible for inclusion and were asked to participate in the trial. Only one BCC per patient was included in order to facilitate the patients' compliance. In patients with two or more BCC eligible for inclusion, the tumour most accessible for topical application of the cream by the patient him/herself was chosen for inclusion. When all BCCs were equally accessible, the largest tumour was included. The main exclusion criteria were: proven or suspected malignancy of other organs; genetic skin cancer disorders; tumors in the face or on the hairy scalp; incapable of comprehending treatment instructions; use of oral NSAIDs ( $>4$ days a week) or vitamin $D$ (containing) supplements during the trial period or within 30 days before inclusion.

\section{Power analysis}

For calculation of the sample size it was assumed that in non-treated BCCs the mean percentage of cells expressing Ki-67 would be $25 \%$ with a standard deviation of $8 \%$. Assuming an alpha of $5 \%$ with a power of $90 \%, 13$ patients per study arm were required to detect a minimally relevant clinical difference of at least $10 \%$ in the percentage of cells expressing Ki-67 at the end of the treatment when comparing active treatment with control. Taking into account a potential lost to follow up of $20 \%$, we planned to include 16 patients per randomized group.

\section{Immunohistochemical staining procedure and assessment}

Formalin-fixed and paraffin-embedded (FFPE) $4 \mu \mathrm{m}$ tissue sections of both biopsy and excision specimens were collected onto one slide to ensure congruent staining results. Immunohistochemistry $(\mathrm{IHC})$ was performed with the primary antibodies Ki-67 and Bcl-2 (both mouse monoclonal antibodies [mAbs]). After heat induced epitope retrieval (K8004 EnVision ${ }^{\mathrm{TM}}$ Flex Target Retrieval Solution High pH 9, DAKO), immunohistochemical analysis was performed using DakoAutostainer Link 48 ${ }^{\mathrm{TM}}$ (DAKO Corporation, Carpinteria, CA, USA). Endogenous peroxidase activity was blocked by peroxidase blocking reagent for 5 minutes. The primary antibodies Ki-67 (Ki-67, clone MIB-1, ready to use Antibody, DAKO) and $\mathrm{Bcl}-2$ ( $\mathrm{Bcl}-2$, clone 124, ready to use Antibody, DAKO) were incubated for 20 minutes. The DAKO Envision Flex kit (K8002) was used for secondary detection. After secondary detection, all sections were counterstained with Haematoxylin. In all IHC procedures positive controls were included. Two independent and blinded dermato-pathologists, manually quantified the percentage of tumor cells positive for Ki-67 and Bcl-2 at x200 magnification. Zero percent expression was scored if there were no tumor cells in the excision specimens (complete histologic tumor regression). Discrepancies in the quantification were resolved in a follow-up joint review of the slides and discussion by both pathologists. 


\section{Comprehensive description of serious adverse events}

The first participant (sBCC, diclofenac sodium-3\% gel) was hospitalized with fever and hypotension associated with a recurrent non-Hodgkin lymphoma. This participant already had a medical history of recurrent Hodgkin lymphomas treated with radio- and chemotherapy more than 5 years ago. The second participant (nBCC, diclofenac sodium-3\% gel) was hospitalized with fever due to a urinary tract infection. The patient had a history of kidney transplantation and known recurrent urinary tract infections. Both patients continued their study medication during their hospitalization. The third participant (nBCC, combined therapy) was hospitalized with fever due to erysipelas at the excision site two days after excision of the target lesion. This participant already discontinued the study medication after trial week 6 due to erythema of the target lesion. The erythema had resolved at the time of the excision. 


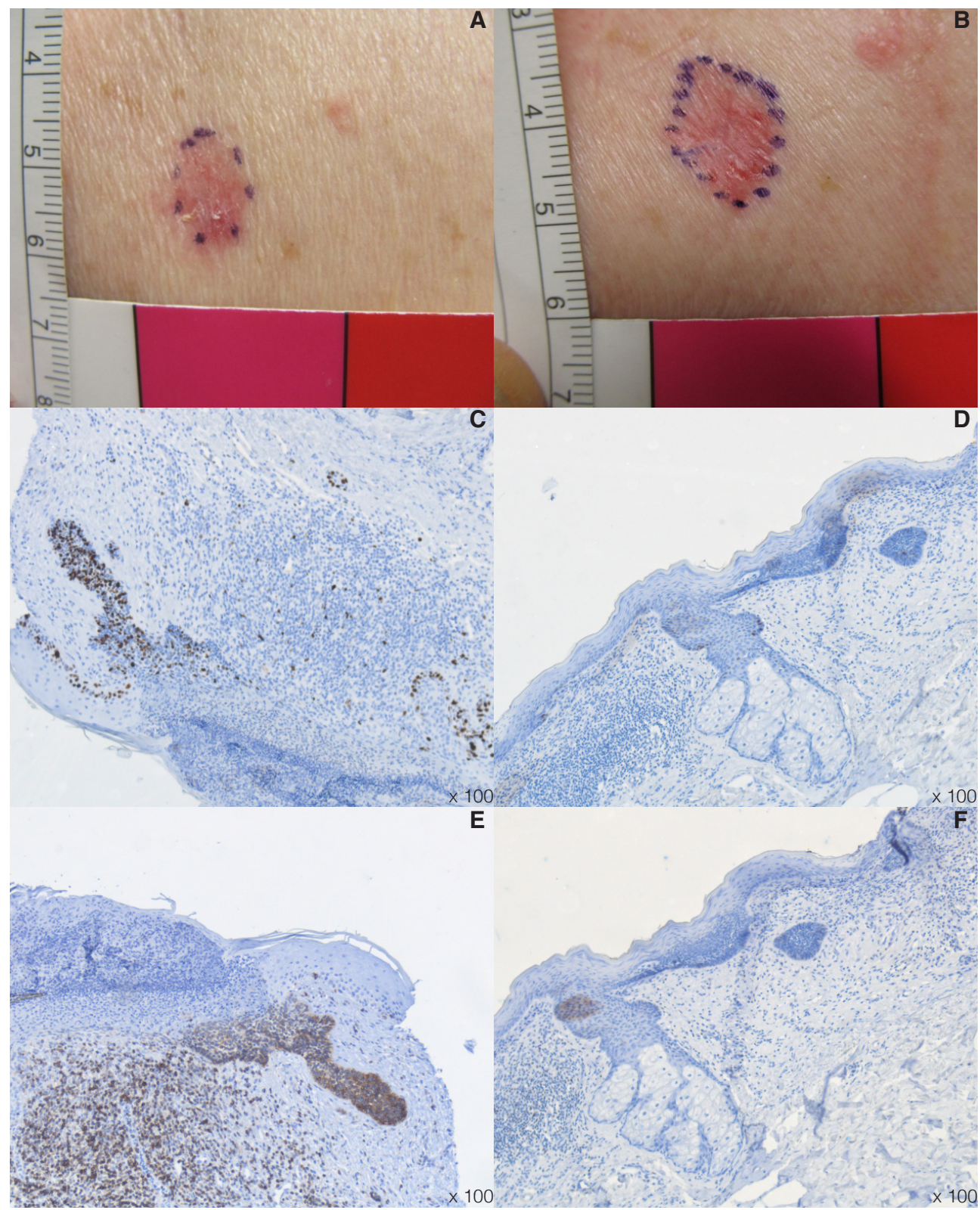

Figure S1. Clinical and histological responses.

A. Superficial basal cell carcinoma (sBCC) at baseline. B. sBCC after 8 weeks treatment with diclofenac sodium-3\% gel and calcitriol $3 \mu \mathrm{g} / \mathrm{g}$ (combined therapy). C. Specific nuclear (brown) Ki-67 expression at baseline in an sBCC. D. Reduction of Ki-67 expression after 8 weeks treatment with combined therapy in an SBCC as assessed by immunohistochemistry (IHC). E. Specific cytoplasmic (brown) Bcl-2 expression at baseline in an sBCC. F. Reduction of Bcl-2 expression after 8 weeks treatment with combined therapy in an sBCC as assessed by IHC. Fig A-F are from the same patient. 


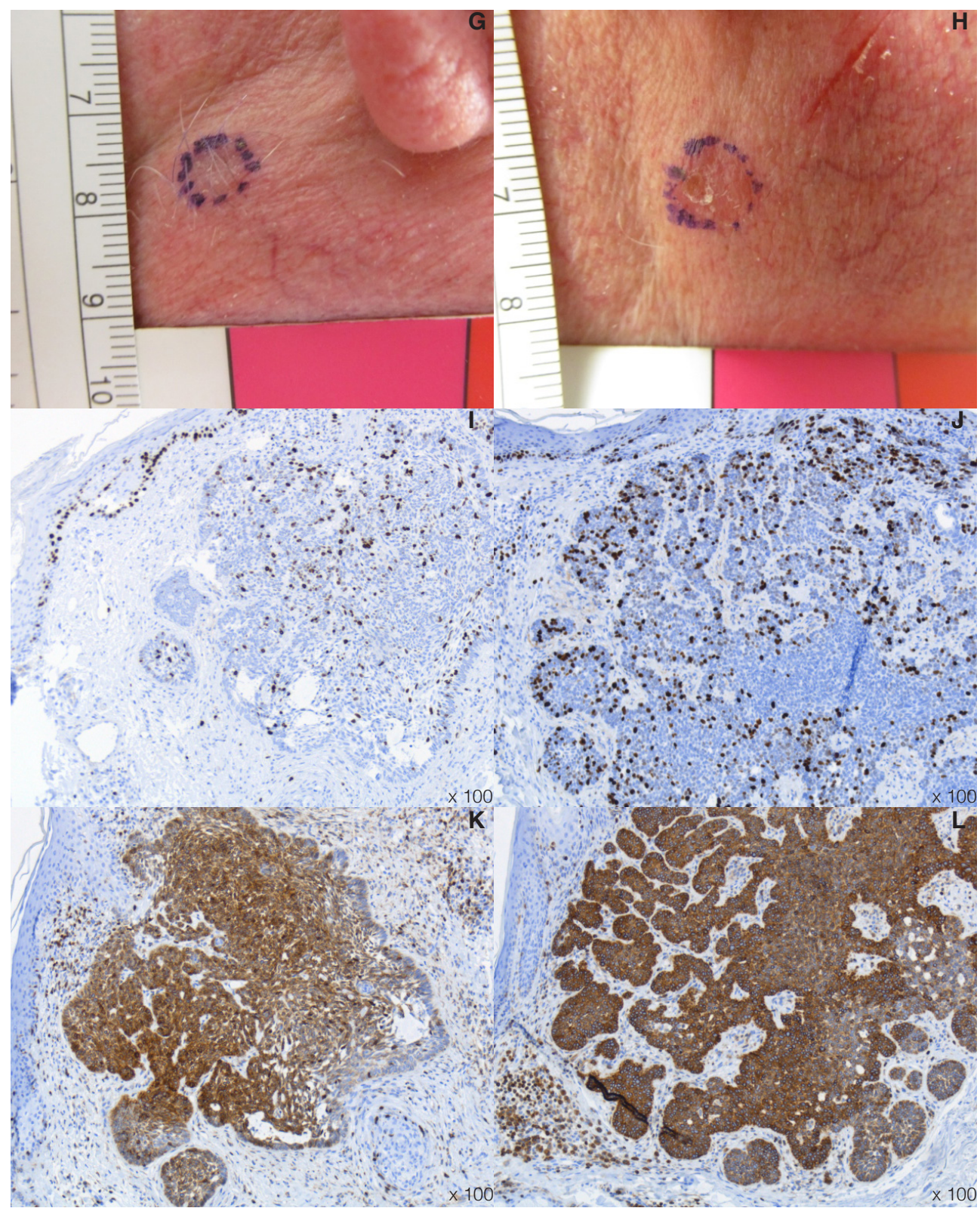

Figure S2. Clinical and histological responses in biopsy versus excision specimens.

G. Nodular basal cell carcinoma (nBCC) at baseline. H. nBCC after 8 weeks without topical treatment (control group). I. Specific nuclear (brown) Ki-67 expression at baseline in an nBCC. J. No reduction of Ki-67 expression after 8 weeks without topical treatment in an nBCC as assessed by immunohistochemistry (IHC). K. Specific cytoplasmic (brown) Bcl-2 expression at baseline in an nBCC. L. No reduction of Bcl-2 expression after 8 weeks treatment without topical treatment in an $\mathrm{nBCC}$ as assessed by $\mathrm{HHC}$. Figures $\mathrm{G}-\mathrm{L}$ are from the same patient. 


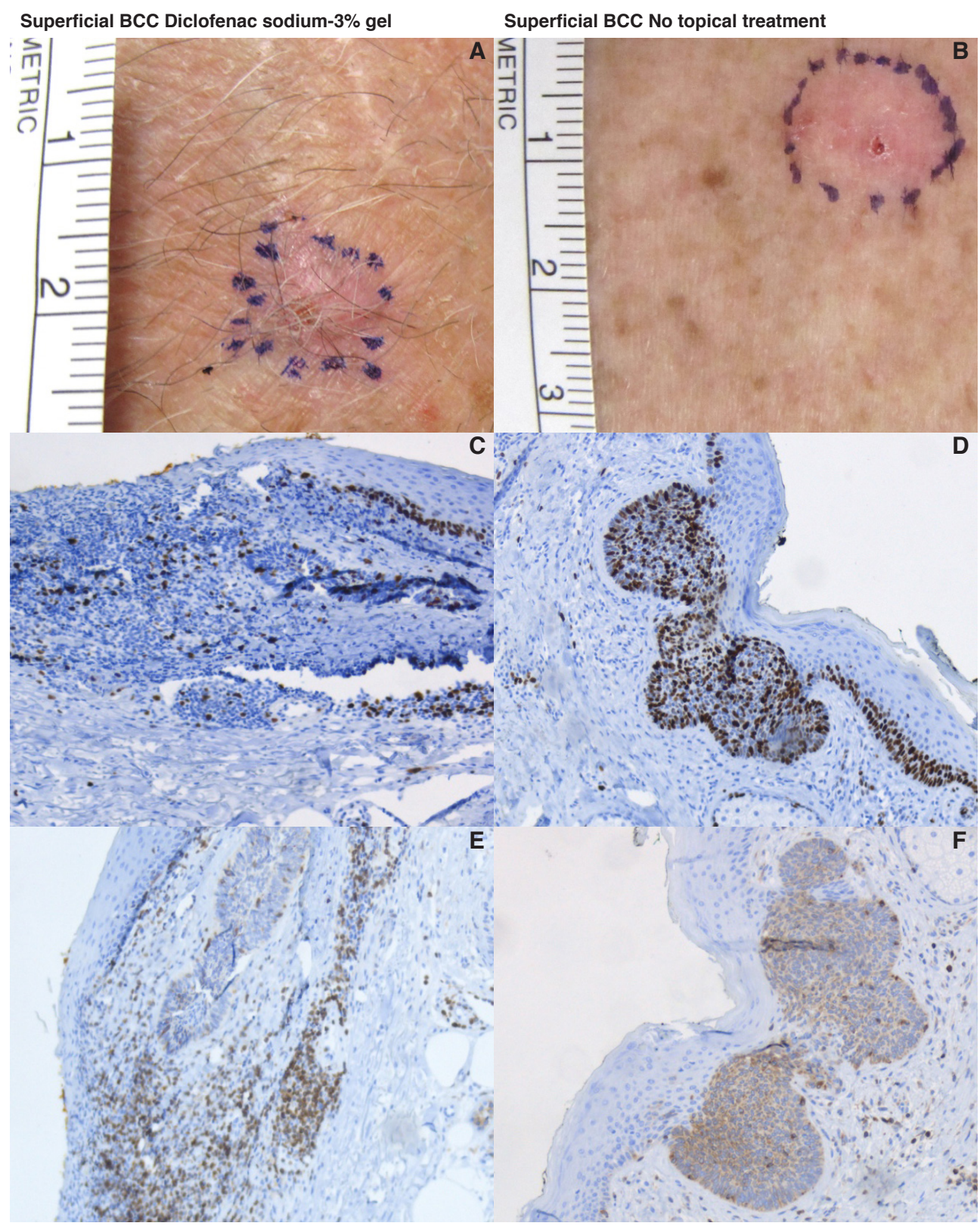

Figure S3. Clinical and histological responses after 8 weeks.

A. Superficial basal cell carcinoma (sBCC) after 8 weeks treatment with topical diclofenac sodium-3\% gel. B. sBCC after 8 weeks without topical treatment (control group). C. Specific nuclear (brown) Ki-67 expression after 8 weeks treatment with diclofenac in an sBCC as assessed by immunohistochemistry (IHC). D. Specific nuclear Ki-67 expression after 8 weeks without topical treatment in an sBCC as assessed by IHC. E. Specific cytoplasmic (brown) Bcl-2 expression after 8 weeks treatment with diclofenac in an sBCC. F. Specific cytoplasmic Bcl-2 expression after 8 weeks without topical treatment in an $\mathrm{BBCC}$ as assessed by $\mathrm{IHC}$. Figures $\mathrm{A}, \mathrm{C}$ and $\mathrm{E}$ and $\mathrm{B}, \mathrm{D}$ and $\mathrm{F}$ are from the same patients. 
Table S1: Median compliance rates according to treatment assignment

Median Compliance (\%)

Superficial BCC

Diclofenac sodium-3\%

Calcitriol

Diclofenac sodium-3\% and Calcitriol
$93.18 \%$ (0 to 100$)$

$98.18 \%(15.45$ to 100$)$

$96.36 \%(10.91$ to 100$)$

Nodular BCC

Diclofenac sodium-3\%

Calcitriol

Diclofenac sodium-3\% and Calcitriol
$91.82 \%$ (23.64 to 100$)$

$96.36 \%$ (0 to 100 )

$93.64 \%$ (0 to 100$)$

Data are median \% (range). BCC=Basal Cell Carcinoma. Diclofenac=diclofenac sodium $-3 \%$ gel, Calcitriol=Calcitriol $3 \mu \mathrm{g} / \mathrm{g}$ ointment, Combined therapy=diclofenac and calcitriol Compliance was calculated as the number of applications actually applied by the patient as percentage of the total prescribed number of applications. 


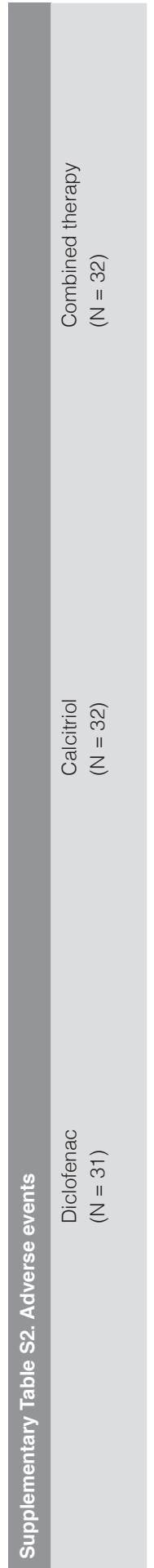

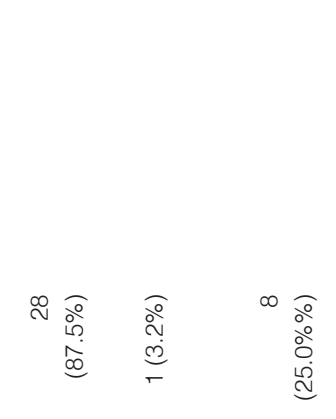

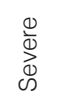

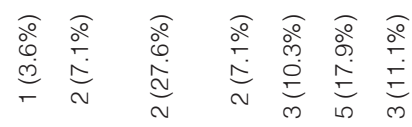

$\frac{0}{\frac{\pi}{\pi}}$

을

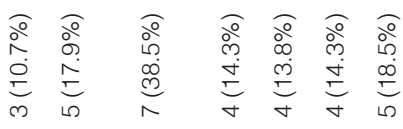

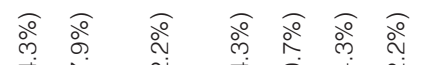

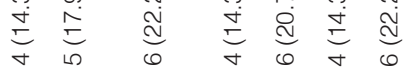

$=\stackrel{\infty}{\sim} \hat{\sim} \quad \stackrel{\sim}{\sim} \stackrel{\infty}{N}$

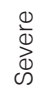

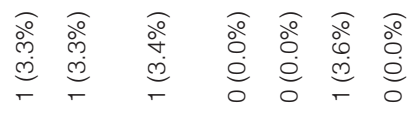

$\frac{0}{\pi}$
$\frac{\pi}{0}$
$\frac{0}{0}$
$\sum$

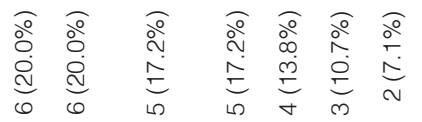

$\stackrel{\frac{0}{\bar{z}}}{\Sigma}$

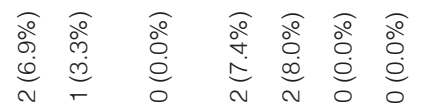

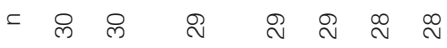

$\stackrel{\frac{1}{d}}{\infty}$

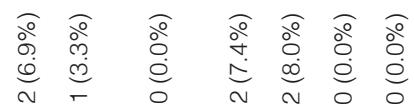

$\frac{0}{\frac{1}{\pi}}$

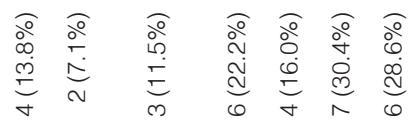

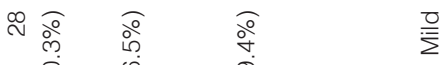

ᄋ $\stackrel{\sim}{0} \quad \stackrel{0}{=}$

$=\stackrel{\sim}{\sim} \stackrel{\sim}{N} \stackrel{N}{\sim}$

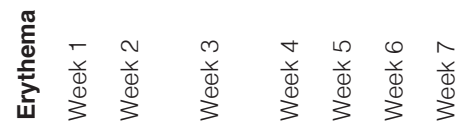

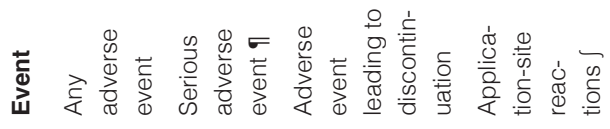




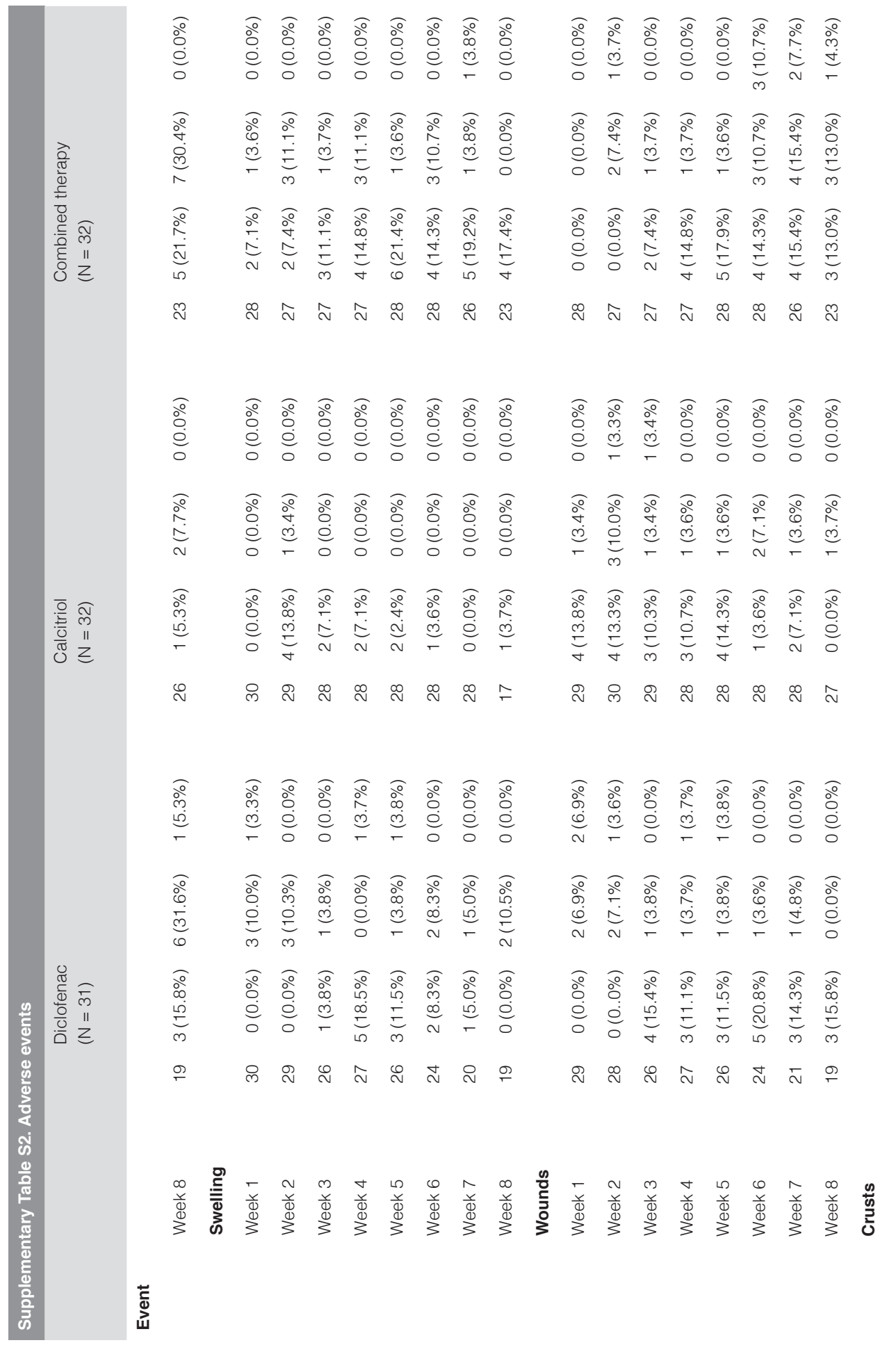




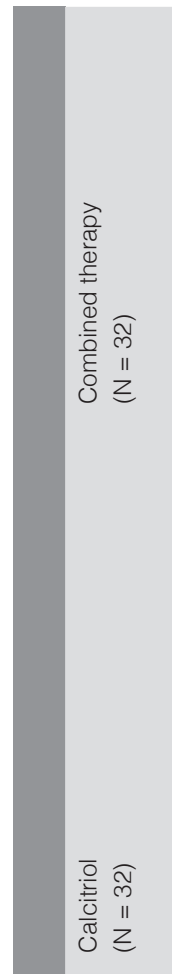

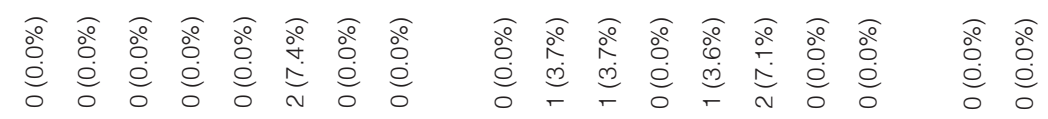

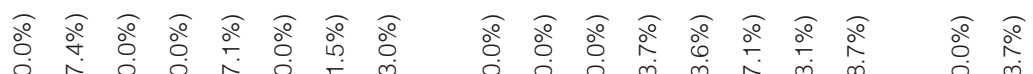

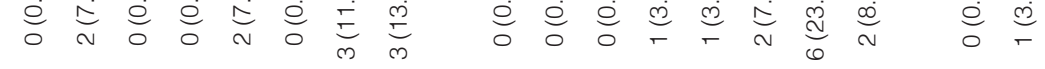

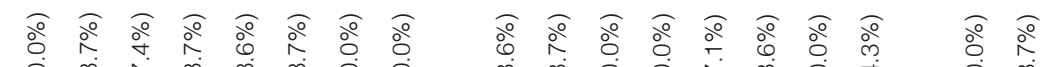

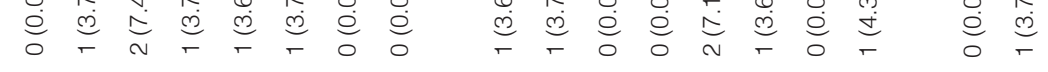

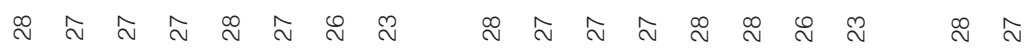

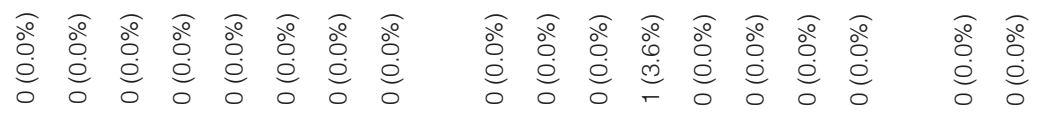

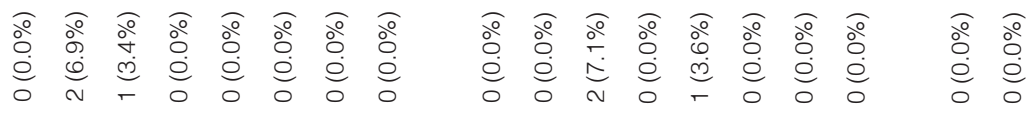

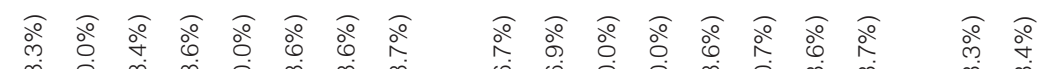

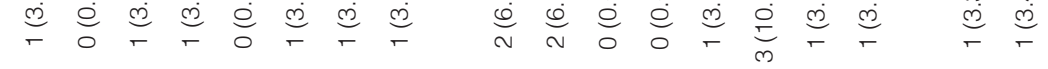

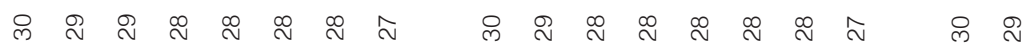

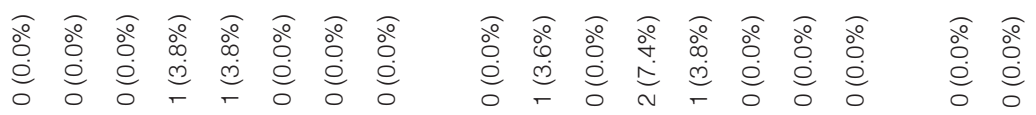

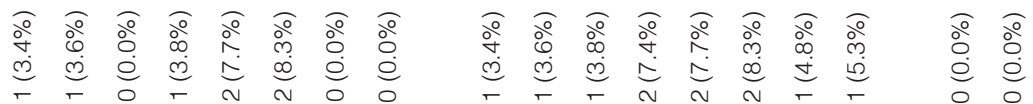

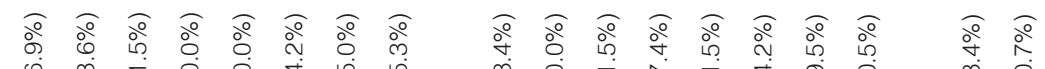

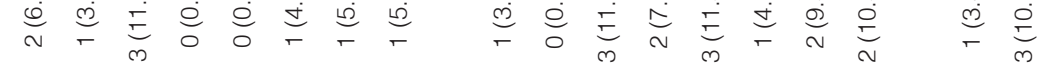

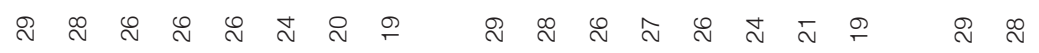

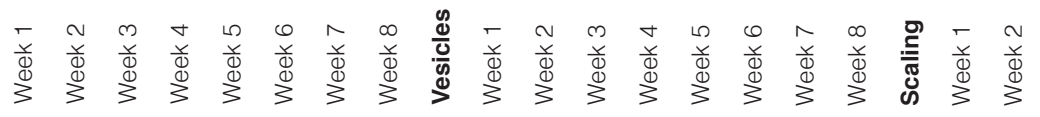




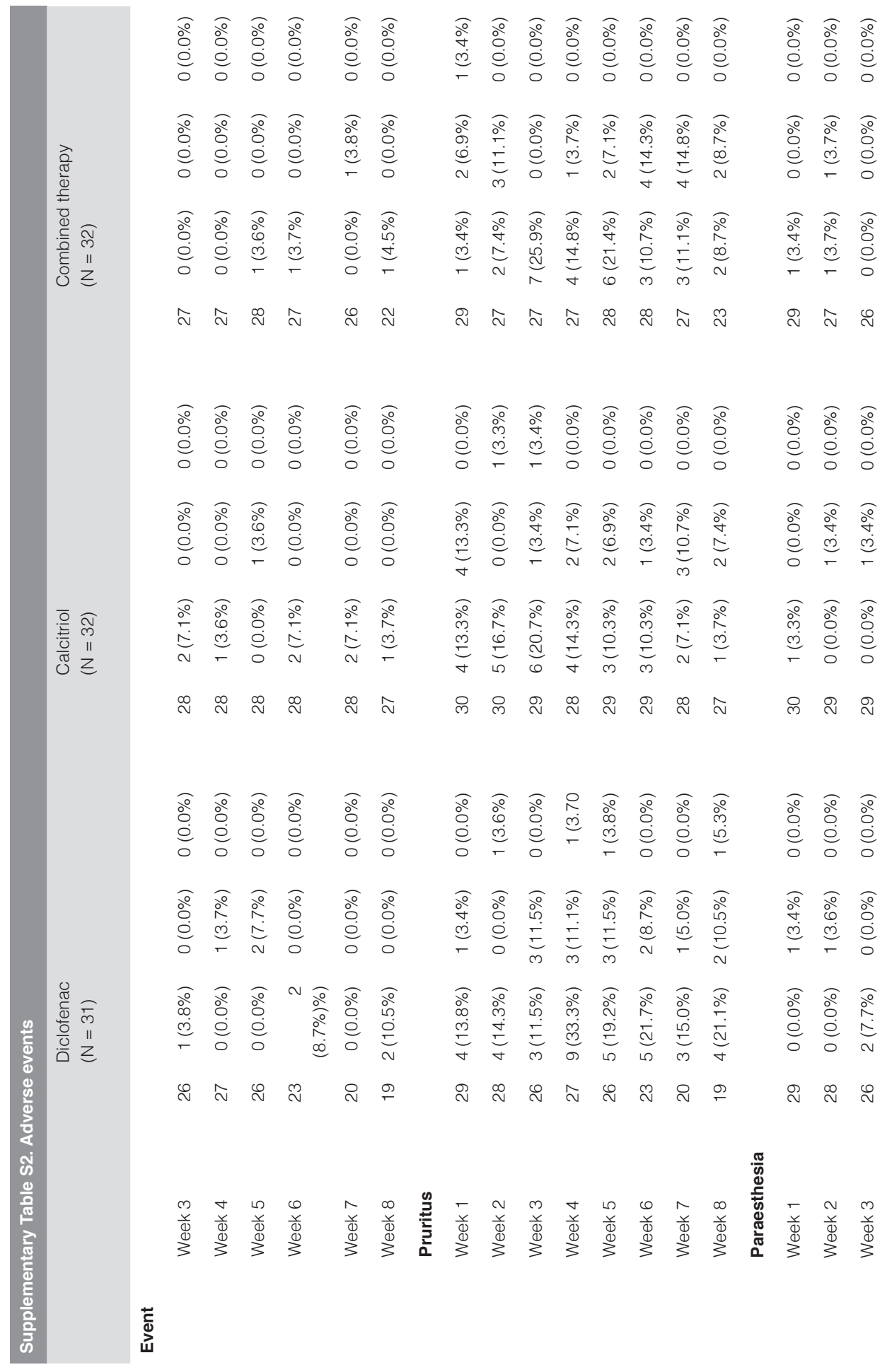




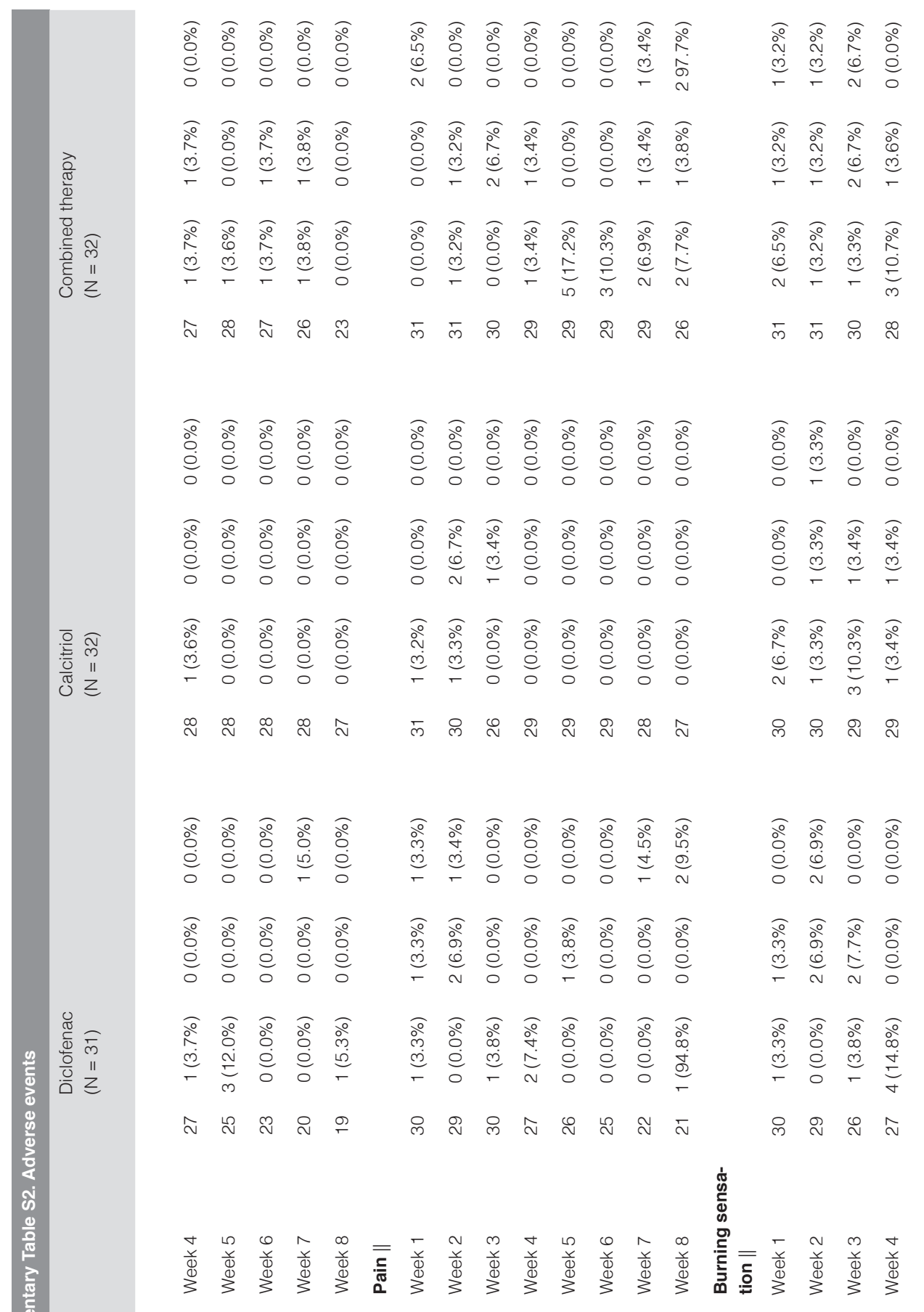




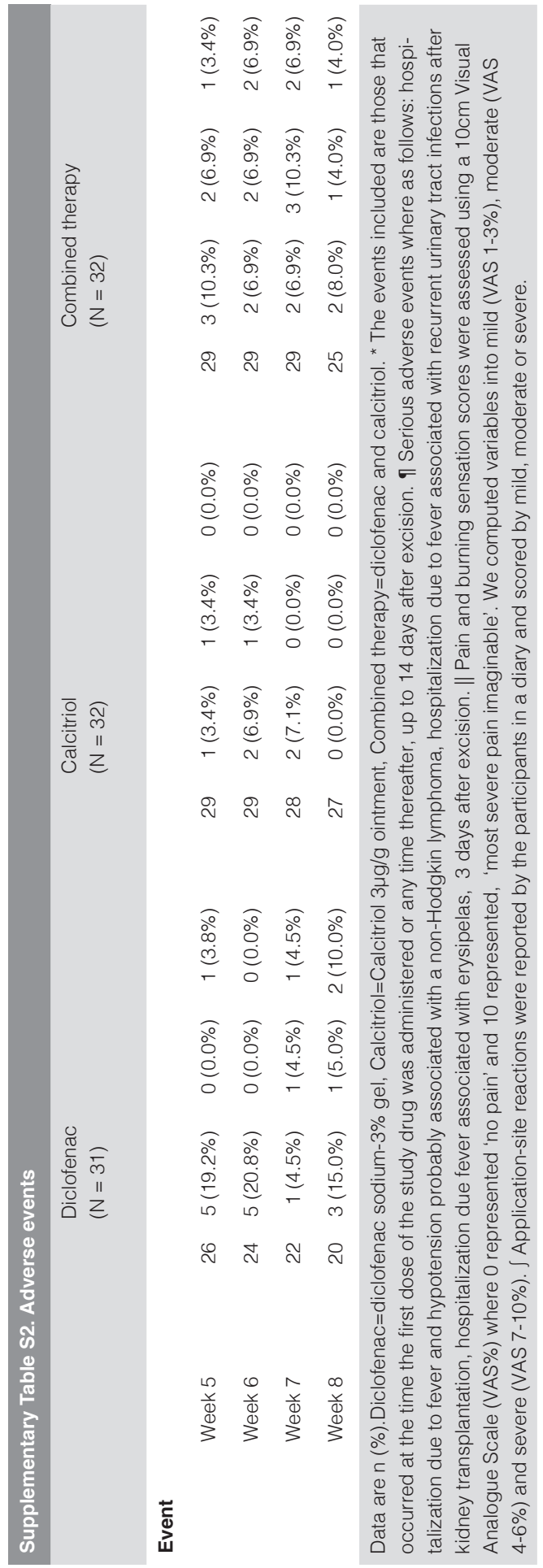





\section{Chapter 4.2}

A comparison of semi-automated versus pathologist-based manual quantification of immunohistochemical stains in basal cell carcinoma

Kiki J.A. Frencken†, Tjinta Brinkhuizen, Annelot J.J. Hendriksen, Jack P.M. Cleutjens, Michiel P.J. van der Horst, Axel Zur Hausen, Véronique J.L. Winnepenninckx, Patty J. Nelemans, Klara Mosterd. 


\begin{abstract}
Immunohistochemical analysis of important markers for proliferation (Ki-67) and apoptosis (Bcl-2) is crucial to evaluate therapeutic efficacy of experimental treatments in basal cell carcinoma (BCC). Manual pathologist-based quantification (MQ) is currently still the most frequently used technique, however it is subjected to high intra- and inter-observer variability, it is time-consuming and labor-intensive. Evaluation of 122 superficial and nodular BCC shows that semi-automated quantification (SAQ) generates reasonable high inter-observer agreements with $95 \%$ limits of agreement between $-9.2 \%$ and $10.8 \%$ for $\mathrm{Ki}-67$ and between $-12.2 \%$ and $20.0 \%$ for Bcl2. However, large discrepancies were seen between SAQ and MQ especially at higher expression levels (more than 20\% positive tumor cells) of $\mathrm{Ki}-67$ and $\mathrm{Bcl}-2$. This finding points to the need for a further study wherein consensus evaluation by more than one pathologist will be compared with SAQ measurements.
\end{abstract}




\section{Introduction}

Immunohistochemical $(\mathrm{IHC})$ analysis is being used more often to gain insight in the underlying mode of action or efficacy of new targeted therapies for basal cell carcinoma (BCC). ${ }^{1-3}$ Manual pathologist-based quantification (MQ) is currently the most frequently used technique for $\mathrm{IHC}$ evaluation ${ }^{4}$ and is considered as the gold standard. However, estimation of the percentage positively stained tumor cells is a subjective approach and may demonstrate high intra- and inter-observer variability. ${ }^{5-7}$ Moreover, $\mathrm{MQ}$ is time-consuming and labor-intensive. ${ }^{8}$ Dichotomized scoring scales are frequently used to standardize quantification of immunohistochemical stains. ${ }^{9,10}$ However, a major disadvantage of these categorized scoring methods is that they are less sensitive to detect limited changes in expression.

BCC is the most common malignancy in Caucasians, leading to a social and economic burden on health care facilities. ${ }^{11,12}$ There is an urgent need for cost-efficient and patient friendly (targeted) non-invasive treatments. The proliferation marker Ki-67 and anti-apoptosis marker $\mathrm{Bcl}-2$ have previously been used in research settings to evaluate therapeutic efficacy of experimental treatments in BCC. ${ }^{1,2}$ The nuclear protein $\mathrm{Ki}-67$ is expressed in all active cell cycle phases, except $\mathrm{G}_{0}{ }^{13} \mathrm{Ki}-67$ is generally accepted as the most accurate cell proliferation marker. ${ }^{14}$ The proto-oncogene Bcl-2 encodes the largest known anti-apoptotic cytoplasmic protein ${ }^{15}$ and inhibits programmed cell death (apoptosis). For reliable evaluation of $\mathrm{IHC}$ analysis in the development of BCC treatments, there is a need for a reproducible and time-efficient method. Digital image analysis techniques offer an objective approach with a high sensitivity for change and the possibility for increased reproducibility. ${ }^{16,17}$ However, comparative studies that prove the assumption of high reproducibility of digital image analysis in BCC are lacking.

A digital image analysis program was used to quantify the expression of $\mathrm{Ki}-67$ and $\mathrm{Bcl}-2 \mathrm{in} \mathrm{BCC}$. The aim of this study was to evaluate reproducibility of semi-automated quantification (SAQ) of skin tissue sections in patients with superficial and nodular BCC using a self-developed software program. Another aim was to evaluate the agreement of SAQ scores with MQ scores. 


\section{Materials \& methods}

\section{Immunohistochemistry}

Tissue sections were derived from patients $(n=128)$ enrolled in a single blind, exploratory phase II, randomized-controlled trial at the Maastricht University Medical Center (MUMC+) registered at clinicaltrials.gov, number NCT01358045 (clinical trial chapter 4.1). All patients gave their written informed consent before enrolment. This study was conducted according to the Declaration of Helsinki principles. Haematoxylin and eosin histological sections from all formalin-fixed paraffin-embedded (FFPE) punch biopsy tumor blocks were re-evaluated by an investigator and a dermato-pathologist and per patient the tumor block including the most tumor cells was selected for further analysis. Immunohistochemical stains were performed on 4- $\mu \mathrm{m}$ tissue sections according to standardized laboratory protocols at the Department of Pathology, MUMC+ by using a DakoAutostainer Link 48 ${ }^{\mathrm{TM}}$ (DAKO Corporation, Carpinteria, CA, USA). Briefly, after heat induced epitope retrieval (K8004 EnVisionTM Flex Target Retrieval Solution High pH 9, DAKO), immunohistochemical analysis was performed using DakoAutostainer Link 48 ${ }^{\mathrm{TM}}$ (DAKO Corporation, Carpinteria, CA, USA). Endogenous peroxidase activity was blocked by peroxidase blocking reagent for 5 minutes. The primary antibodies Ki-67 (IR614, clone MIB-1, mouse monoclonal, ready to use Antibody, DAKO) and Bcl-2 (IR626, clone 124, mouse monoclonal, ready to use Antibody, DAKO) were incubated for 20 minutes. The DAKO Envision Flex kit (K8002) was used for secondary detection. After secondary detection, all sections were counterstained with haematoxylin. In both $\mathrm{IHC}$ procedures positive controls were included and all showed the expected positive result.

\section{Quantification of immunohistochemical staining}

One dermato-pathologist and two investigators analyzed the same set of 122 biopsies stained with $\mathrm{Ki}-67$ and $\mathrm{Bcl}-2$ antibodies. MQ was performed independently by one dermato-pathologist blinded for order, patient and clinical data. BCC nests were identified manually in all fields. The percentage positively stained tumor cells (brown staining) within the total number of tumor cells was manually estimated in all tumor nests on the entire slide at x200 magnification.

SAQ was performed independently by two trained, but previously inexperienced, investigators blinded for order, patient and clinical data. All slides were analyzed by using a so-called operator-dependent computerized thresholding technique (Leica Qwin version 3.5.1, Leica Microsystems, Cambridge, UK). This technique is an extensively evaluated method, especially for mammographic density. ${ }^{18,19}$ All tumor nests were manually identified under low-power magnification. From each section, two color photomicrographs of 2088×1550 pixels redgreen-blue (RGB) format were obtained with a Leica DFC320® digital camera coupled to a microscope (Leica DM3000) using a x10 objective for both markers. In case there was only one tumor nest on the biopsy, one picture was taken. The images were stored as tagged image file (TIF). For each new session the microscope, camera and computer were calibrated according to a standardized procedure. Both stains were performed on parallel tissue sections. All images were analyzed using Leica Qwin version 3.5.1. For each image, the following 
procedure was performed. The investigator defined the tumor area to be quantified by encircling the area manually with a mouse. Color thresholds were manually calibrated to establish a positive color threshold for each immunohistochemical staining. The threshold values of each staining were held constant during the assessment of all images. The percentage nuclear (Ki-67) or cytoplasm (Bcl-2) immunopositive area was calculated automatically by dividing the sum of the pixels of the immunopositive area by the sum of the pixels of the total tumor area multiplied by one hundred. Outcomes were automatically stored in an Excel-file.

\section{Statistical analysis}

Statistical analyses were performed by using SPSS version 20.0 (IBM SPSS Statistics, Chicago, IL, USA). Agreement between observers on SAQ scores as well as agreement between $S A Q$ and $M Q$ measurements was visualized by Bland-Altman plots. The difference in measurements between observers are plotted against the mean of measurements. ${ }^{20}$ If the mean difference deviates from zero, this deviance can be interpreted as systematic error. The standard deviation (SD) of the mean difference is used to calculate $95 \%$ limits of agreement $(1.96 \times \mathrm{SD})$ which represent the range wherein $95 \%$ of discrepancies between measurements are situated. The limits of agreement give an indication of the size of random measurement error. ${ }^{21}$ Additionally, the inter-observer reproducibility for SAQ was calculated by using the intra-class correlation coefficient (ICC). An ICC $<0.4$ indicates poor reproducibility, $\geq 0.4$ and $<0.75$ fair to good reproducibility and $\geq 0.75$ excellent reproducibility. ${ }^{22}$

\section{Results}

Semi-automated quantification shows reproducible expression levels of Ki-67 and Bcl-2 in basal cell carcinoma

Of 128 available punch biopsy specimens, 122 (58 superficial BCC and 64 nodular $\mathrm{BCC}$ ) included sufficient paraffin embedded tumor tissue to enable staining with Ki-67 and Bcl-2 antibodies, respectively. All 122 slides were assessed by SAQ and MQ. Figure 1 provides an overview of an example of a superficial/nodular BCC assessed using an operator-dependent computerized thresholding technique (Leica Qwin version 3.5.1).

For Ki-67, mean percentage of positive tumor cells was $16.2 \pm 10.0$ for investigator 1 and $15.4 \pm 10.3$ for investigator 2 (Table 1). A Bland-Altman plot of the SAQ results for Ki-67 indicates a mean difference between investigators of $0.8 \pm 5.1$ with $95 \%$ limits of agreement ranging from -9.2 to 10.8 (Table 2 and Figure $2 a$ ). The intra-class correlation coefficient (ICC) was 0.87 [95\% Cl 0.82-0.91].

Mean expression levels of Bcl-2 were $12.9 \pm 13.8$ for investigator 1 and $9.0 \pm 10.5$ for investigator 2 (average $10.9 \pm 11.6$ ) (Table 1). The mean difference between the two assessors was $3.9 \pm 8.2$. The $95 \%$ limits ranged from -12.2 to 20.0 . The ICC was of 0.74 [95\% CI 0.58-0.84] (Table 2 and Figure 2b). 


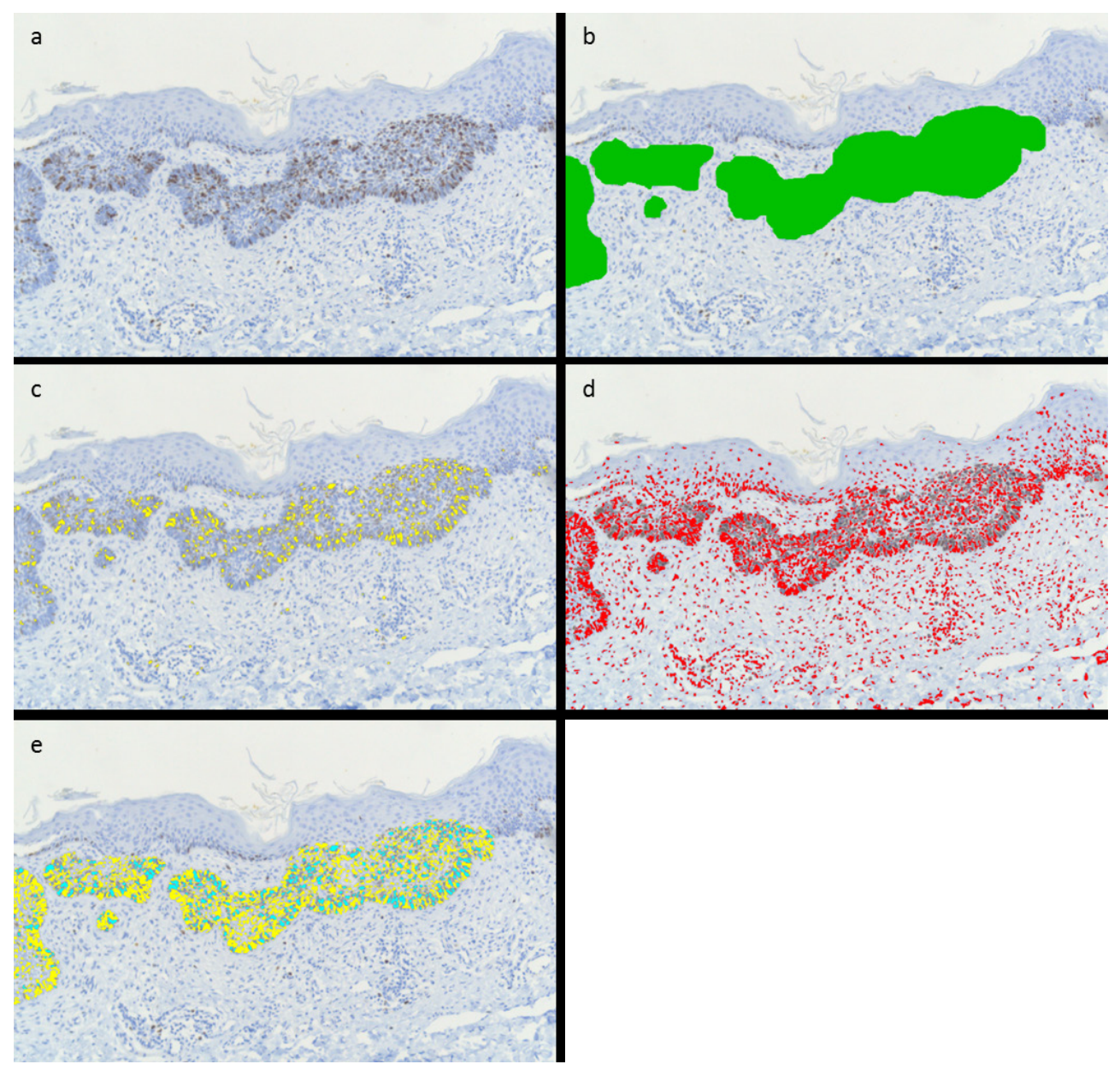

Figure 1. Overview of operator-dependent computerized thresholding technique (Leica Qwin version 3.5.1, Leica Microsystems, Cambridge, UK) for Ki-67 immunohistochemical stains.

An overview of Ki-67 semi-automated quantification in a basal cell carcinoma using an operator-dependent computerized thresholding technique. (a) Immunohistochemical stain for Ki-67 in a superficial and nodular basal cell carcinoma. (b) Manually drawn tumor area. (c) Detection of positively stained tumor cells. (d) Detection of negatively stained tumor cells. (e) Positively and negatively stained cells per within the tumor area. 
Table 1. Percentages of positively stained cells for semi-automated (SAQ) and manual quantification (MQ) measurements.

$\begin{array}{lrr}\text { Ki-67 } & \text { Mean } \% \pm \text { SD } & \begin{array}{r}\text { Average of mean \% SAQ scores } \\ \pm S D\end{array} \\ \text { SAQ investigator 1 } & 16.2 \pm 10.0 & 15.8 \pm 9.8 \\ \text { SAQ investigator 2 } & 15.4 \pm 10.3 & 19.3 \pm 20.1 \\ \text { MQ } & & 10.9 \pm 11.6 \\ \text { Bcl-2 } & 12.9 \pm 13.8 & 9.0 \pm 10.5 \\ \text { SAQ investigator 1 } & & 34.6 \pm 32.9 \\ \text { SAQ investigator 2 } & & \end{array}$

Table 2. Agreement between semi-automated quantification (SAQ) derived by two investigators and agreement between $S A Q$ and manual quantification (MQ) by one pathologist.

$\begin{array}{lrr}\text { SAQ (investigator1 vs investigator 2) } & \text { Mean difference } \pm \text { SD } & \text { 95\% limits of agreement } \\ \text { Ki-67 } & 0.8 \pm 5.1 & -9.2 \text { to } 10.8 \\ \text { Bcl-2 } & 3.9 \pm 8.2 & -12.2 \text { to } 20.0 \\ \text { MQ minus SAQ } & 3.5 \pm 14.4 & -24.7 \text { to } 31.7 \\ \text { Ki-67 } & -2.2 \pm 7.4 & -16.7 \text { to } 12.3 \\ \text { Ki-67 } \leq 20 \% \text { positive cells } & 19.0 \pm 17.3 & -14.9 \text { to } 52.9 \\ \text { Ki-67 > 20\% positive cells } & 23.7 \pm 26.8 & -28.8 \text { to } 76.2 \\ \text { Bcl-2 } & 3.3 \pm 9.2 & -14.7 \text { to } 21.3 \\ \text { Bcl-2 } \leq 20 \% \text { positive cells } & 51.0 \pm 16.0 & 19.64 \text { to } 82.36 \\ \text { Bc-2 }>20 \% \text { positive cells } & \end{array}$

SD: standard deviation of mean difference. The results presented are percentages. 

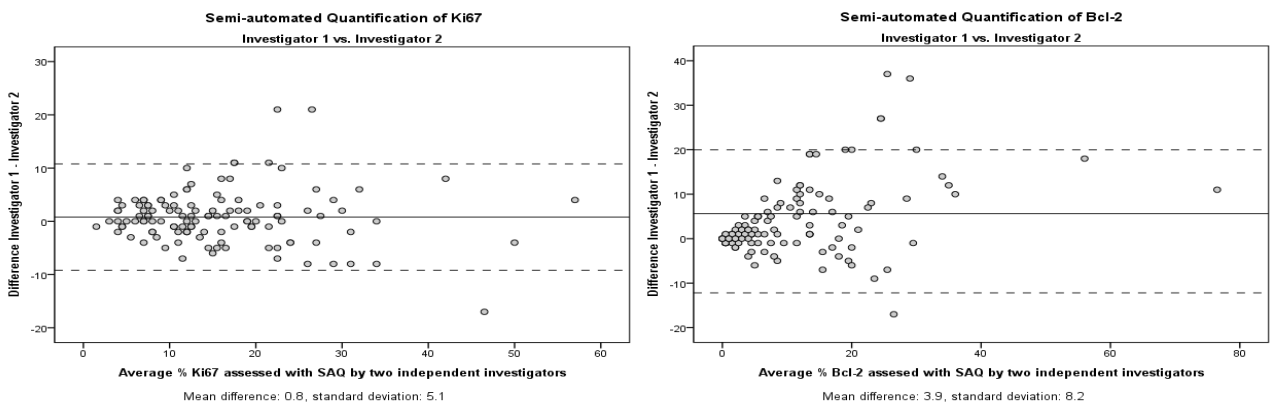

Figure 2. Bland-Altman plot for semi-automated quantification of $\mathrm{Ki}-67$ and $\mathrm{Bcl}-2$ immunohistochemical stains in basal cell carcinoma showing high reproducibility.

Semi-automated quantification by two independent investigators for Ki-67, (a) and Bcl-2, (b). The average percentage positive tumor cells is represented on the $\mathrm{x}$-axis. The $\mathrm{y}$-axis (difference in $\mathrm{Ki}-67$ and $\mathrm{Bcl}-2$ respectively) represents the difference in percentage of positively stained tumor cells assessed by assessor 1 minus assessor 2. The middle continuous line represents the mean difference of all measurements. The two dashed lines represent the $95 \%$ limits of agreements $( \pm 1.96$ Standard Deviation).

Manual quantification versus semi-automated quantification of Ki-67 and Bcl-2 in basal cell carcinoma shows limited agreement, especially at high percentage positive tumor cells

When comparing SAQ with $\mathrm{MQ}$, the mean percentage of Ki-67 positive cells according to $M Q$ was $19.3 \pm 20.1 S D$ and higher than the average of the two investigators on SAQ of $15.8 \pm 9.8$ (Table 1). The mean difference was $3.5 \pm 14.4$ and 95\% of the differences fell between -24.7 and 31.7 with (Table 2 and Figure 3a). However, the Bland-Altman plot indicated that the magnitude of differences did not remain the same over the whole range of mean values. Limited agreement was seen especially at higher expression levels of Ki67. Therefore, tumors were categorized into subgroups according to percentage of positive tumor cells. A cut-off point of $20 \%$ was chosen. Within tumors with $\leq 20 \%$ positive cells, $\mathrm{MQ}$ was scored lower than SAQ (mean difference $-2.2 \pm 7.4$ ) with $95 \%$ limits of agreement between -16.7 and 12.3) (Table 2 and Figure 3b). Within tumors with $>20 \%$ positive cells higher scores for MQ compared to SAQ were seen with a mean difference of $19.0 \pm 17.3$ ) and wider $95 \%$ limits of agreement ranging from -14.9 to 52.9) (Table 2 and Figure 3c). 

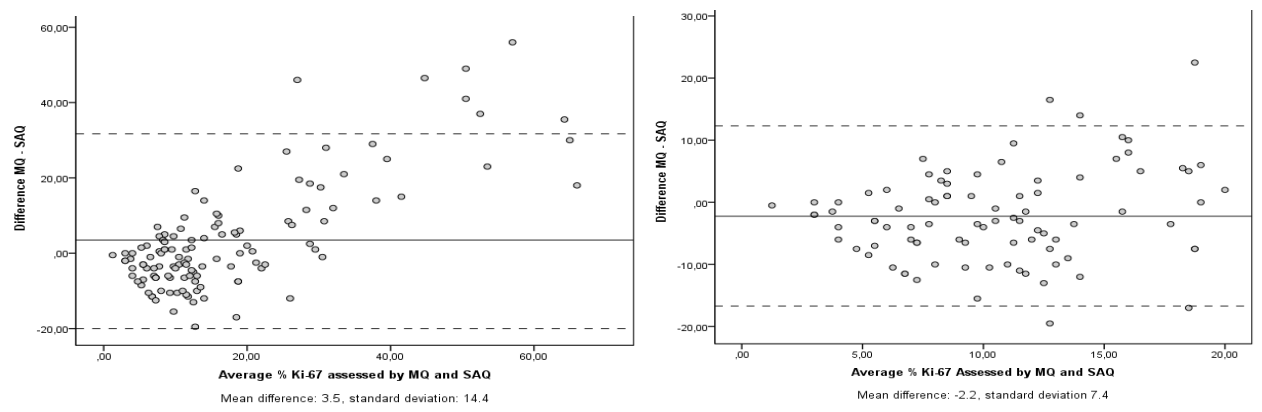

C

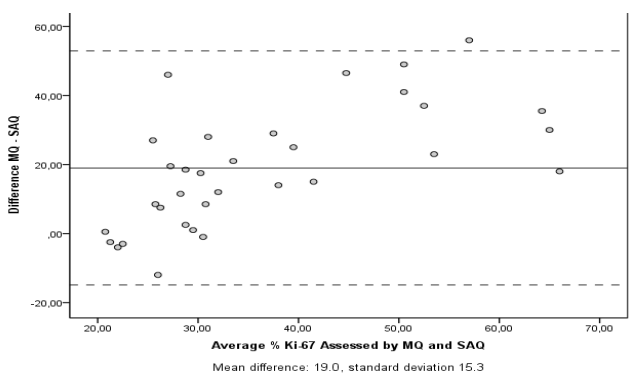

Figure 3. Bland-Altman plot for manual quantification versus semi-automated quantification of $\mathrm{Ki}-67 \mathrm{immu}-$ nohistochemical stains in basal cell carcinoma showing variable agreement, especially at high percentages of positive cells.

Manual quantification (MQ) by one pathologist versus semi-automated quantification (SAQ) of the average of two independent investigators for Ki-67. (a) Overall results. (b) Results separately for percentages of positive tumor cells $\leq 20 \%$. (c) Results separately for percentages of positive tumor cells $>20 \%$. (a, b, c) The average percentage positive tumor cells is represented on the $x$-axis. The $y$-axis represents the difference in percentage of positively stained tumor cells assessed by MQ minus SAQ. The middle continuous line represents the mean difference of all measurements. The two dashed lines represent the $95 \%$ limits of agreements ( \pm 1.96 Standard Deviation).

For Bcl-2, the mean percentage positive cells according to $\mathrm{MQ}$ was $34.6 \pm 32.9$ versus an average of $10.9 \pm 11.6$ according to SAQ (Table 1). The mean difference was $23.7 \pm$ 26.8 and $95 \%$ of the differences fell between -28.8 and 76.2. Again, differences between $\mathrm{SAQ}$ and $\mathrm{MQ}$ were seen especially at high expression levels of Bcl-2 (Table 2, Figure $4 \mathrm{a}$ ). In BCC with $\leq 20 \%$ positive tumor cells, the mean difference was $3.3 \pm 9.2$ (95\% limits of agreement between -14.7 and 21.3) (Table 2, Figure 4b). The mean difference was much higher within the subgroup with $>20 \%$ positively stained cells (MD 51 \pm 16.0 ) and the $95 \%$ limits of agreement were wide (19.64 to 82.36) Table 2, Figure 4c). 

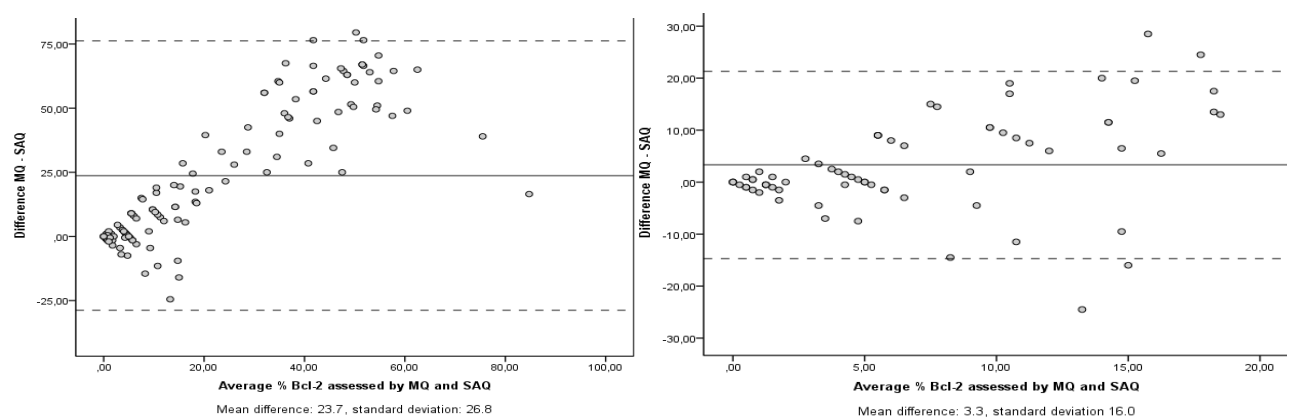

C

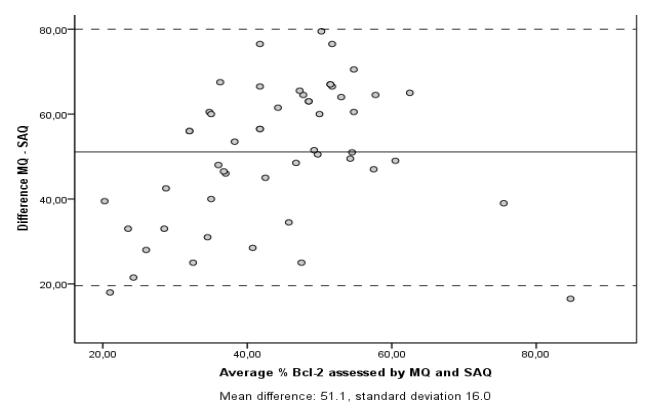

Figure 4. Bland-Altman plot for manual quantification versus semi-automated quantification of Bcl-2 immunohistochemical stains in basal cell carcinoma showing variable agreement, especially at high percentages of positive cells.

Manual quantification (MQ) by one pathologist versus semi-automated quantification (SAQ) of the average of two independent investigators for Bcl-2. (a) Overall results. (b) Results separately for percentages of positive tumor cells $\leq 20 \%$. (c) Results separately for percentages of positive tumor cells $>20 \%$. (a, b, c) The average percentage positive tumor cells is represented on the $x$-axis. The $y$-axis represents the difference in percentage of positively stained tumor cells assessed by MQ minus SAQ. The middle continuous line represents the mean difference of all measurements. The two dashed lines represent the $95 \%$ limits of agreements ( \pm 1.96 Standard Deviation). 


\section{Discussion}

In this study, we used a dedicated software program that we have developed to evaluate the reproducibility of SAQ of superficial and nodular BCC cells expressing Ki-67 and Bcl-2, and we compared SAQ scores with MQ scores. Our results show that the $95 \%$ limits of agreement between SAQ scores of two observers lie between -9.2\% and 10.8\% for Ki-67 and between $-12.2 \%$ and $20.0 \%$ for $\mathrm{Bcl} 2$. The random measurement error may be acceptable for evaluation of treatments with large effects. However, in case of studies on therapies with more subtle effects it may be advisable to use the average score of two observers to increase the precision.

When we compared $S A Q$ to $M Q$, large discrepancies between $S A Q$ and $M Q$ scores were observed especially in tumors with higher levels of expression of Ki-67 or Bcl2. In tumors with a mean of $\geq 20 \%$ positively stained cells, the $M Q$ score tended to be systematically higher than the SAQ score. Whether this should be considered as overestimation of $\mathrm{MQ}$ or underestimation of SAQ remains debatable. True reference values are required in order to determine the accuracy of a measurement, but for expression of Ki-67 and Bcl-2 in BCC no true reference values are available. Although the evaluation by the pathologist is still considered as the gold standard, the results obtained in our study suggest that MQ could overestimate the amount of positive cells. This is line with literature describing that positive cells are more prone to capture the attention of the assessor as compared to unstained cells. ${ }^{23}$ It has previously been reported that when comparing $M Q$ and $S A Q$ in breast tissue, higher scores for $\mathrm{MQ}$ were mainly seen in highly positive tissue. ${ }^{19}$ In our study, discrepancies were even larger for Bcl-2, which is a cytoplasmic stain and cytoplasmic stains might be even harder to asses manually because the distinction with "background staining" can be difficult.

Another explanation for the lack of agreement between $S A Q$ and $M Q$ could be related to a variation in the selection of tumor area to be counted. As BCC are characterized by (multiple) small nests of basaloid cells spreading into the epidermis, $M Q$ is often based on the estimation of positive cells in a limited area of the tumor in one or a few fields of view. Then, this estimation is averaged and extrapolated to the total tumor nest. Since it is known that BCC is a highly heterogeneous tumor, this approach may not be representative for the total of all tumor nests. ${ }^{24}$ Visual recognition remains a subjective method and, even for the experienced pathologist, the assessment is performed in a more approximate rather than an accurate manner. ${ }^{16,25} \mathrm{SAQ}$ has the advantage of measuring a large area of positive cells, negative cells and stromal tissue. In case of BCC, all tumor nests will be included in the measurement. This large number of evaluated cells reduces the risk of over- or underestimation. Furthermore the computer objectively measures the percentage positively stained tumor cells.

Another major advantage of SAQ is that even investigators with little experience in using the software, can achieve excellent reproducibility, ${ }^{19}$ as is also confirmed in this study. Additionally, the software program can obtain extra measurements like a quantitative staining intensity measurement or other details, which can help to distinguish for example background staining from surrounding positive tissues. All measurements are automatical- 
ly stored in an Excel-file, which make the data easy accessible for statistical analyses. In contrast to fully automated quantifications, SAQ, with the technique we used, is dependent on the selection of color thresholds by the observer. However, the threshold is set once before the start of the measurements and is saved for all following slides, so in case of different observers, this would lead to a systematic difference between observers rather than random measurement errors. Some authors warn for the fact that the software program also requires input from the observer for tumor selection. However, human input as in SAQ even seems to be necessary since a human observer may be better at recognizing non-tumor or stromal areas in the sample than a fully automated image analysis system. ${ }^{26}$

There are some limitations of the study. Currently, assessment by the pathologist is still regarded as the gold standard in the assessment of immunohistochemical stains, but assessment of $\mathrm{MQ}$ by only one pathologist is a considerable disadvantage of this study. Because of the lack of agreement between $S A Q$ and $M Q$ measurements, we were not able to derive definite conclusions on accuracy of SAQ. Further study is needed which enable comparison of SAQ scores with MQ scores by multiple pathologists. Such a study design not only offers the opportunity to evaluate the reproducibility of pathologist based measurements and to compare it with reproducibility of SAQ scores but also provides a more valid approach to the concept of assessment by the pathologist as gold standard. Evidence from a well-designed study that would confirm that SAQ is a reproducible and accurate method for assessing immunohistochemical stains is very relevant for research. The use of a semi-automated technique would be especially useful when large amounts of slides needs to be assessed, which is often the case in clinical trials studying new treatment modalities. Pre- and post-treatment expression levels of important immunohistochemical markers are more and more used as important outcome measures. Assessing the change in expression values is less subjected to random errors of individual observers which makes SAQ very suitable for these studies. Even the rather inexperienced young researches would be able to generate valid results and at and this could relieve the workload of the pathologist in the daily practice of patients diagnostics.

In this study, reasonable high inter observer agreement was demonstrated for both Ki-67 and $\mathrm{Bcl}-2$ antibody assessment with SAQ. The large discrepancies between SAQ and MQ measurements can partly be explained by systematic error. Systematic error is not a large problem, because adjustment for this type of error is easy. More worrying are the wide $95 \%$ limits of agreement especially in highly positive tumors. This finding points to the need for studies wherein consensus evaluation by more than one pathologist will be compared with SAQ measurements. 


\section{References}

1. Cho EA, Moloney FJ, Cai H, et al. Safety and tolerability of an intratumorally injected DNAzyme, Dz13, in patients with nodular basal-cell carcinoma: a phase 1 first-in-human trial (DISCOVER). Lancet 2013;381:183543.

2. Kim DJ, Kim J, Spaunhurst K, et al. Open-label, exploratory phase II trial of oral itraconazole for the treatment of basal cell carcinoma. Journal of clinical oncology : official journal of the American Society of Clinical Oncology 2014;32:745-51.

3. Tang JY, Mackay-Wiggan JM, Aszterbaum M, et al. Inhibiting the hedgehog pathway in patients with the basal-cell nevus syndrome. The New England journal of medicine 2012;366:2180-8.

4. Camp RL, Chung GG, Rimm DL. Automated subcellular localization and quantification of protein expression in tissue microarrays. Nature medicine 2002;8:1323-7.

5. Taylor CR, Levenson RM. Quantification of immunohistochemistry--issues concerning methods, utility and semiquantitative assessment II. Histopathology 2006;49:411-24.

6. Rubin MA, Zerkowski MP, Camp RL, et al. Quantitative determination of expression of the prostate cancer protein alpha-methylacyl-CoA racemase using automated quantitative analysis (AQUA): a novel paradigm for automated and continuous biomarker measurements. The American journal of pathology 2004;164:83140.

7. Polley MY, Leung SC, McShane LM, et al. An international Ki-67 reproducibility study. Journal of the National Cancer Institute 2013;105:1897-906.

8. Daniel K, Maria A, Amelie L, et al. Somatostatin receptor immunohistochemistry in neuroendocrine tumors: comparison between manual and automated evaluation. International journal of clinical and experimental pathology 2014;7:4971-80.

9. Allred DC, Harvey JM, Berardo M, Clark GM. Prognostic and predictive factors in breast cancer by immunohistochemical analysis. Modern pathology : an official journal of the United States and Canadian Academy of Pathology, Inc 1998;11:155-68.

10. de Jong D, Rosenwald A, Chhanabhai M, et al. Immunohistochemical prognostic markers in diffuse large B-cell lymphoma: validation of tissue microarray as a prerequisite for broad clinical applications--a study from the Lunenburg Lymphoma Biomarker Consortium. Journal of clinical oncology : official journal of the American Society of Clinical Oncology 2007;25:805-12.

11. Housman TS, Feldman SR, Williford PM, et al. Skin cancer is among the most costly of all cancers to treat for the Medicare population. Journal of the American Academy of Dermatology 2003;48:425-9.

12. Lomas A, Leonardi-Bee J, Bath-Hextall F. A systematic review of worldwide incidence of nonmelanoma skin cancer. The British journal of dermatology 2012;166:1069-80.

13. Gerdes J, Lemke H, Baisch H, Wacker HH, Schwab U, Stein H. Cell cycle analysis of a cell proliferation-associated human nuclear antigen defined by the monoclonal antibody Ki-67. Journal of immunology 1984;133:1710-5.

14. Dowsett M, Nielsen TO, A'Hern R, et al. Assessment of Ki-67 in breast cancer: recommendations from the International Ki-67 in Breast Cancer working group. Journal of the National Cancer Institute 2011;103:165664.

15. Vidal D, Matias-Guiu X, Alomar A. Efficacy of imiquimod for the expression of Bcl-2, Ki-67, p53 and basal cell carcinoma apoptosis. The British journal of dermatology 2004;151:656-62.

16. Feuchtinger A, Stiehler $\mathrm{T}$, Jutting $U$, et al. Image analysis of immunohistochemistry is superior to visual scoring as shown for patient outcome of esophageal adenocarcinoma. Histochemistry and cell biology 2014.

17. Tang LH, Gonen M, Hedvat C, Modlin IM, Klimstra DS. Objective quantification of the Ki-67 proliferative index in neuroendocrine tumors of the gastroenteropancreatic system: a comparison of digital image analysis with manual methods. The American journal of surgical pathology 2012;36:1761-70.

18. Yaffe MJ. Mammographic density. Measurement of mammographic density. Breast cancer research : BCR 2008;10:209. 
19. Lobbes MB, Cleutjens JP, Lima Passos V, et al. Density is in the eye of the beholder: visual versus semi-automated assessment of breast density on standard mammograms. Insights into imaging 2012;3:91-9.

20. Bland JM, Altman DG. Statistical methods for assessing agreement between two methods of clinical measurement. Lancet 1986;1:307-10.

21. de Vet HCWT, C.B.; Mokkink, L.B.; Knol, D.L. . Measurement in Medicine. A practical guide.: Cambridge University Press; 2011.

22. Rosner B. Fundamentals of Biostatistics. 6th ed. Duxbury, Belmont2006.

23. Fasanella S, Leonardi E, Cantaloni C, et al. Proliferative activity in human breast cancer: Ki-67 automated evaluation and the influence of different Ki-67 equivalent antibodies. Diagnostic pathology 2011;6 Suppl $1:$ S7.

24. Jee BA, Lim H, Kwon SM, et al. Molecular classification of basal cell carcinoma of skin by gene expression profiling. Molecular carcinogenesis 2014.

25. Dobson L, Conway C, Hanley A, et al. Image analysis as an adjunct to manual HER-2 immunohistochemical review: a diagnostic tool to standardize interpretation. Histopathology 2010;57:27-38.

26. Konsti J, Lundin M, Joensuu H, et al. Development and evaluation of a virtual microscopy application for automated assessment of Ki-67 expression in breast cancer. BMC clinical pathology 2011;11:3. 
A comparison of semi-automated vs pathologist-based manual quantification of immunohistoch. stains in BCC | 157 



\section{Chapter 5.1}

Acquired Resistance to the Hedgehog Pathway Inhibitor Vismodegib due to smoothened Mutations in Treatment of locally advanced basal cell carcinoma

Tjinta Brinkhuizen*, Marie G. Reinders*, Michel van Geel, Annelotte J.J. Hendriksen, Aimee D. Paulussen, Véronique J.L. Winnepenninckx, Kristien B. Keymeulen, Patricia M Soetekouw, Maurice A.M. van Steensel, Klara Mosterd.

*Both authors contributed equally to this paper and should be considered as first authors.

Published as a Letter to the Editor Journal of the American Academy of Dermatology 2014 Nov; 71(5):1005-8. 


\section{Abstract}

Background: Basal cell carcinoma (BCC) is the most common cancer in Caucasians. Aberrant activation of the Sonic Hedgehog $(\mathrm{SHH})$ signaling pathway is a key event in the development of BCCs. Smoothened (SMO) activates $\mathrm{SHH}$ signaling and can be inhibited by small molecule inhibitors, such as vismodegib. However subsequent acquired resistance is an upcoming problem.

Objective: To analyze the acquired resistance in a 68-year old woman with a locally advanced BCC, who developed tumor regrowth 20 weeks after an initial response to vismodegib.

Methods: Mutation analysis of PTCH1 and SMO was performed on primary tumor tissue as well as on secondary developed tumors and on clinically responding skin.

Results: Histologic examination confirmed the presence of BCC in the newly developed tumors. A PTCH1 mutation was found both in the primary tumor and in the two newly developed tumor nodules. The recurrent tumor nodules harbored two SMO mutations ( $p$.Trp281Leu and $p$. Val321Met) which were neither found in the primary BCC nor in the responding skin.

Limitation: We performed no functional analysis.

Conclusion: Our findings suggest that in this patient, two novel acquired SMO mutations resulted in resistance of the BCC to vismodegib. Alternating treatment schedules or combined therapies can be promising in this respect. 


\section{Introduction}

Sporadic basal cell carcinoma (BCC) is the most frequent malignancy in Caucasians. It mostly behaves indolently, however when neglected it can become very extensive and destructive. Such locally advanced BCC (laBCC) are usually located in the head and neck area and surgical excision is the primary choice of treatment. ${ }^{1,2}$ However, surgery can be very mutilating or disabling and radiotherapy as alternative treatment is not always desirable. ${ }^{2}$ BCC metastases are rare (0.003 to $0.55 \%$ ), usually affecting lymph nodes, liver or lungs, and are characterized by a poor prognosis with median survival rates of 8-14 months. ${ }^{3}$ Reports of successful treatment with cisplatin based chemotherapy for laBCC and metastatic BCC (mBCC) are scarce. ${ }^{4}$

The SHH pathway is essential for embryonic development and in adult tissues it regulates tissue stem cells required for organ repair and maintenance. ${ }^{5}$ Upregulation of $\mathrm{SHH}$ signaling is crucial in the development of almost all BCCs and also plays a role in the pathogenesis of other human malignancies, such as brain and pancreatic cancer (Fig A and B in the Supplementary Appendix). ${ }^{6}$ Hedgehog ligands bind to the tumorsuppressor transmembrane receptor protein Patched homologue $1(\mathrm{PTCH} 1)$, suppressing inhibition of the oncogenic transmembrane protein Smoothened (SMO) and so activates downstream effectors. ${ }^{5}$ Approximately $90 \%$ of sporadic BCCs have identifiable mutations in $\mathrm{PTCH}$, and an additional $10 \%$ have activating mutations in $S M O{ }^{5}$

In 2012 the U.S. Food and Drug Administration (FDA) approved vismodegib 150 mg capsules for the treatment of laBCC and $\mathrm{mBCC}{ }^{7}$ Vismodegib is an orally active synthetic cyclopaminelike SMO-inhibitor. In a phase 2 study, complete and partial response to vismodegib was seen in 43\% (laBCC) and 30\% (mBCC) of patients, respectively ${ }^{8}$ and recent follow-up results showed increasing response rates of respectively $47.6 \%$ and $33.3 \%{ }^{9}$ Initial response to vismodegib followed by secondary tumor progression has already been observed. ${ }^{10-14}$ Acquired resistance to vismodegib has also been described in medulloblastoma, caused by a heterozygous SMO mutation found in the metastatic medulloblastoma that developed during treatment. ${ }^{15}$ Here, we describe a patient with IaBCC who developed resistance to vismodegib after an initial response. To unravel the mechanism of this resistance, we performed mutation analysis on pre-treatment tumor tissue as well as on responding tissue and recurrent tumors after treatment with vismodegib. 


\section{Case description}

A 68-year-old woman presented with an extensive, deeply ulcerating tumor on the dorsal side of her left shoulder, with a diameter of 15 centimeters, which initially started as a small asymptomatic red papule twenty years ago twenty years ago (Fig. 1A). She had never sought medical care before. Histological examination showed an infiltrative BCC (Fig. $2 \mathrm{~A}$ ) and magnetic resonance imaging (MRI) confirmed extensive tumor invasion of the shoulder girdle, including the infraspinatus, teres major and minor and also in the proximal latissimus dorsi muscles and a spina scapulae fragment with part of the deltoid muscle (Fig 1B, 1C). There was no evidence for lymphogenic or visceral metastasis. Because curative surgery would imply a significant risk of impaired shoulder function, our patient decided to participate in a trial with vismodegib. She was enrolled in a clinical trial and treated with vismodegib $150 \mathrm{mg}$ once daily. Treatment resulted in dramatic clinical tumor regression within 8 weeks of treatment. After 16 weeks, a complete clinical response was achieved; only sclerotic tissue remained (Fig 1D). Treatment was continued, but at a control visit in week 20 multiple nodules were found in the former tumor area (FIG $1 \mathrm{E})$. On MRI multifocal tumor recurrence was seen in the skin and underlying muscles (Fig 1F, 1G). Biopsies of two of these nodules revealed infiltrative BCC while in clinically responding skin no $\mathrm{BCC}$ cells were detected (Fig. $2 \mathrm{~B}$ and $2 \mathrm{C}$ ). Because of this progressive disease, vismodegib was discontinued approximately 26 weeks after treatment initiation. Extensive surgical excision was performed, including resection of involved musculature, resulting in tumor free margins. The defect was successfully closed with a rotation flap.

For mutation analyses, 8-mm biopsy samples were taken from both clinically tumor-free sclerotic tissue and two newly developed tumor nodules during surgery. Each (tumor) tissue sample was coated with Tissue-Tek® O.C.T. (Sakura) and immediately frozen and stored at $-80^{\circ} \mathrm{C}$. From all tissue samples a $4 \mathrm{~mm}$ section was stained with haematoxylin \& eosin (H\&E) as part of routine diagnostic procedure. All H\&E sections were reviewed by a dermato-pathologist to confirm diagnosis and histological subtype. We collected a saliva sample (OrageneDNA, DNA GenoTek, Kanata, Ontario, Canada). Written informed consent was obtained. 

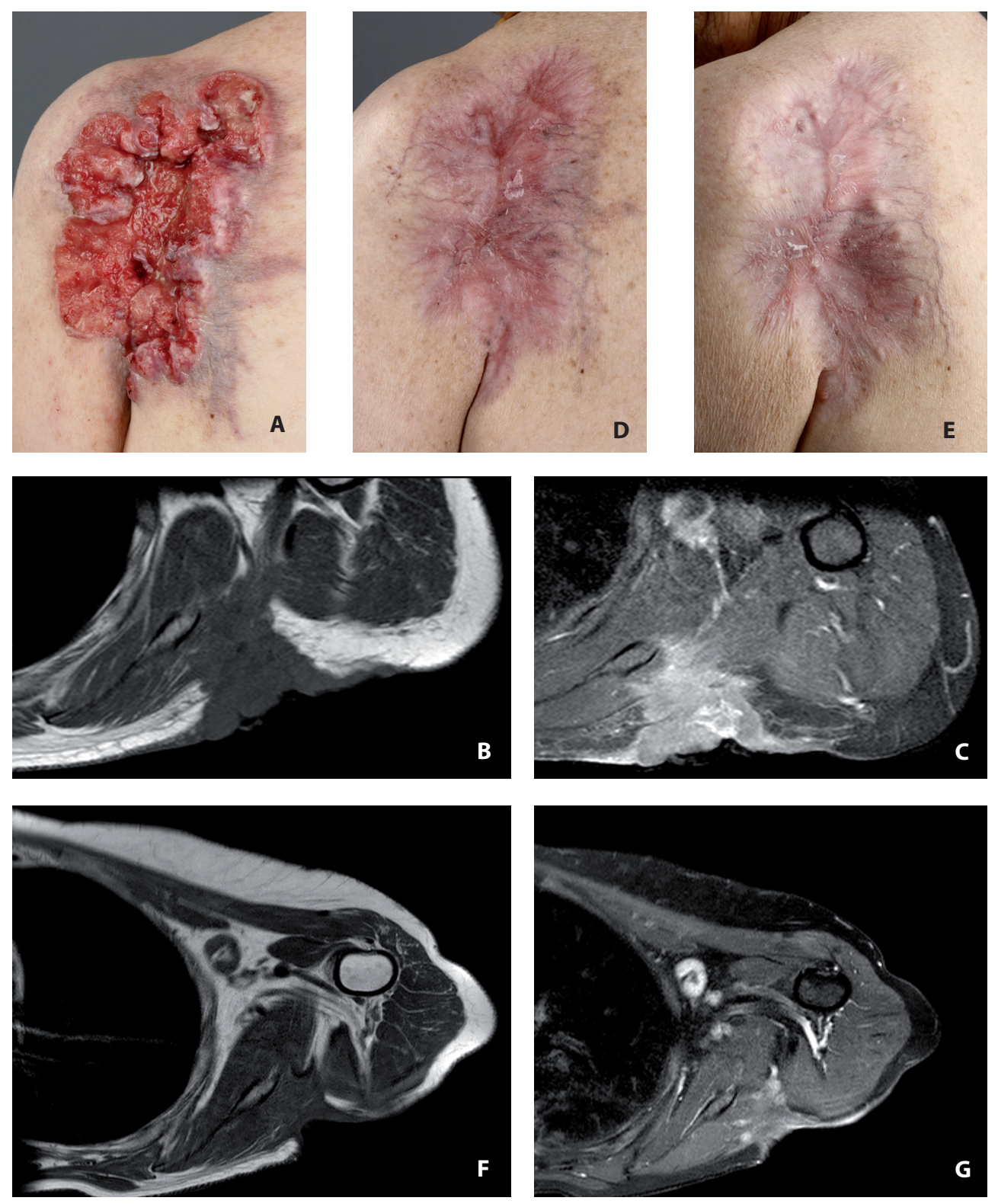

Figure 1. Clinical and magnetic resonance imaging (MRI) measured response of the tumor.

A. Clinical picture basal cell carcinoma (BCC) before treatment with vismodegib. B. Corresponding T1 MRI and T1 with fat suppression (Spectral Presaturation with Inversion Recovery (SPIR)) MRI after gadolinium are shown in panel C. D. Complete granulation of defect, 16 weeks after start of treatment. E. Newly developed tumors after 27 weeks of treatment with vismodegib and in panel $\mathbf{F}$ and $\mathbf{G}$ the corresponding T1 MRI and T1 SPIR MRI after Gadolinium. 

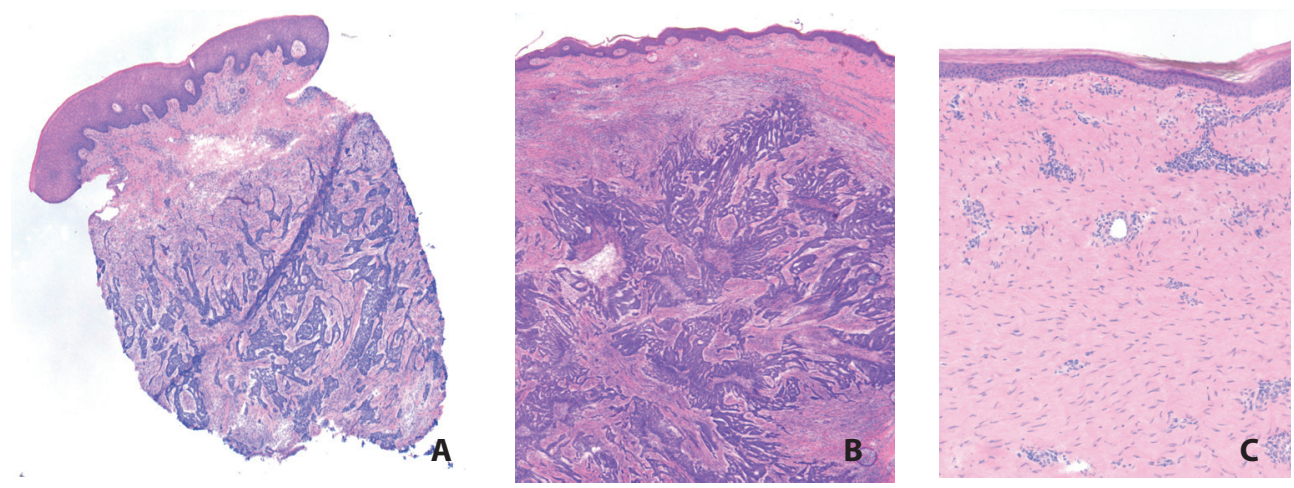

Figure 2: Histopathological examination of pre- and post-treatment tissue.

A. Tumor biopsy before treatment with vismodegib, showing infiltrative palisading basaloid cells reaching into the reticular dermis fitting the diagnosis of BCC (haematoxylin-eosin (HE) magnification x2,5). B. Biopsy from newly developed tumor nodule after 27 weeks of treatment with vismodegib, showing infiltrative basal cell carcinoma. (HE, magnification 2,5x). C. Biopsy of responding tissue after 27 weeks of vismodegib, showing extensive fibrosis with an increased number of fibroblasts, neovascularization, a sparse lymphocytic infiltrate, but no residual BCC (HE, magnification $\times 2,5)$.

\section{Materials en methods}

\section{Nucleic acid extraction}

Genomic DNA was extracted from fresh frozen (FF) biopsy tissue by using NucleoSpin® Tissue XS (Machery-Nagel) and from saliva by using Oragene-DNA kit (DNA GenoTek), following the manufacturer's directions.

\section{Mutation analysis}

All coding exons and immediate flanking intron sequences of PTCH1 were analysed by PCR (AmpliTaq 360, Life Technologies Europe BV, Bleiswijk Netherlands) and direct sequencing (BigDye® Terminator v1.1 Cycle Sequencing Kit and 3730 DNA Analyzer, Life Technologies) in DNA isolated from a primary tumor biopsy sample (protocol and primer sequences available on request). Targeted sequence analysis of the detected PTCH1 mutation was tested in all other tissue samples.

Loss of heterozygosity $(\mathrm{LOH})$ was confirmed in the primary tumor with allele specific sequencing by using cloning of the PCR product (PGEM-T Easy cloning systems, Promega Benelux, Leiden, Netherlands) and quantitation of the specific cloned allele by PCR and subsequent restriction fragment analysis with BstUI (New England BioLabs, Whitby, ON, Canada), digesting the C-allele of the rs2066836 SNP. Sequence data of the cloned fragments showed that the C-allele of the SNP is linked to the PTCH1 deletion/insertion mutation. 
All coding exons and immediate flanking intron sequences of SMO were analysed by PCR (Taq DNA polymerase, New England Biolabs)and direct sequencing in DNA isolated from secondary tumor biopsy samples (protocol and primer sequences available on request). Targeted sequence analysis of the detected SMO mutations was tested in all other tissue samples.

\section{Results}

Histologic examination of biopsies of the primary tumor as well the two newly developed nodules revealed infiltrative BCC (Fig. 2.). The biopsies of the sclerotic tissue showed extensive fibrosis with an increased number of fibroblasts, neovascularization, a sparse lymphocytic infiltrate, but no residual BCC (Fig 2C). Genomic analysis of the PTCH1 coding exons in DNA isolated from the primary BCC identified a heterozygous c.1728_1728+1delinsAA mutation (GenBank RefSeq NM_000264.3) (Fig. 3), putatively affecting the splice donor site of intron12 (5 different splice site prediction programs included in Alamut version 2.3) (Fig 2 in the Supplementary Appendix). As expected, this mutation was not present in DNA isolated from buccal mucosa (saliva). Interestingly, a heterozygous polymorphism present in exon 12 (dbSNP 137: rs2066836) showed an equal peak distribution in buccal mucosa while the pattern was skewed $(\mathrm{T}<\mathrm{C})$ in tumor tissue. Similar skewed peak distributions in tumor DNA were noted at different location within the PTCH1 gene (dbSNP 137: rs2297088, rs2297086, rs2066829, rs2274692, rs2236406). This skewed allelic distribution may indicate loss of heterozygosity $(\mathrm{LOH})$. DNA sequencing is not a quantitative method; therefore the PTCH1 exon 12 PCR amplicon derived from the tumor was cloned and quantified by linking the mutation to the predominant SNP C-haplotype. In 15\% of clones (5 out of 33 clones) the wild type allele linked to the SNP T-haplotype was found, clearly showing underrepresentation of this allele and thus being indicative of $\mathrm{LOH}$. The PTCH1 mutation was found in the two newly developed tumor nodules (Fig. 3.), as was the skewed nucleotide distribution. In the sclerotic tissue (2 biopsies) remaining after treatment with vismodegib, the c.1728_1728+1delinsAA mutation was not present and the flanking rs2066836 SNP showed the same allelic distribution as buccal mucosa. Vismodegib resistance in medulloblastoma is caused by acquired mutations in $S M O^{14-17}$ and we reasoned that similar mutations might occur in BCCs to confer resistance. The coding exons of the SMO gene (GenBank RefSeq NM_005631.4) were analyzed in the two biopsy samples from the newly developed tumors, unveiling a heterozygous C.842G>T mutation (p.Trp281Leu) in exon 4 in one recurrent BCC nodule. The other BCC nodule revealed a heterozygous c.961G >A mutation (p.Val321Met) in exon 5 (Fig 4.). Both missense mutations are novel and are located respectively in the second and third transmembrane domain of the smoothened protein. The importance of these amino acid residues is reflected by the fact that they are completely conserved between species. Neither mutation was found in the laBCC biopsy specimen obtained before initiation of vismodegib therapy, in the biopsy specimens of sclerotic skin after discontinuation of therapy or in the buccal swab (not shown). 

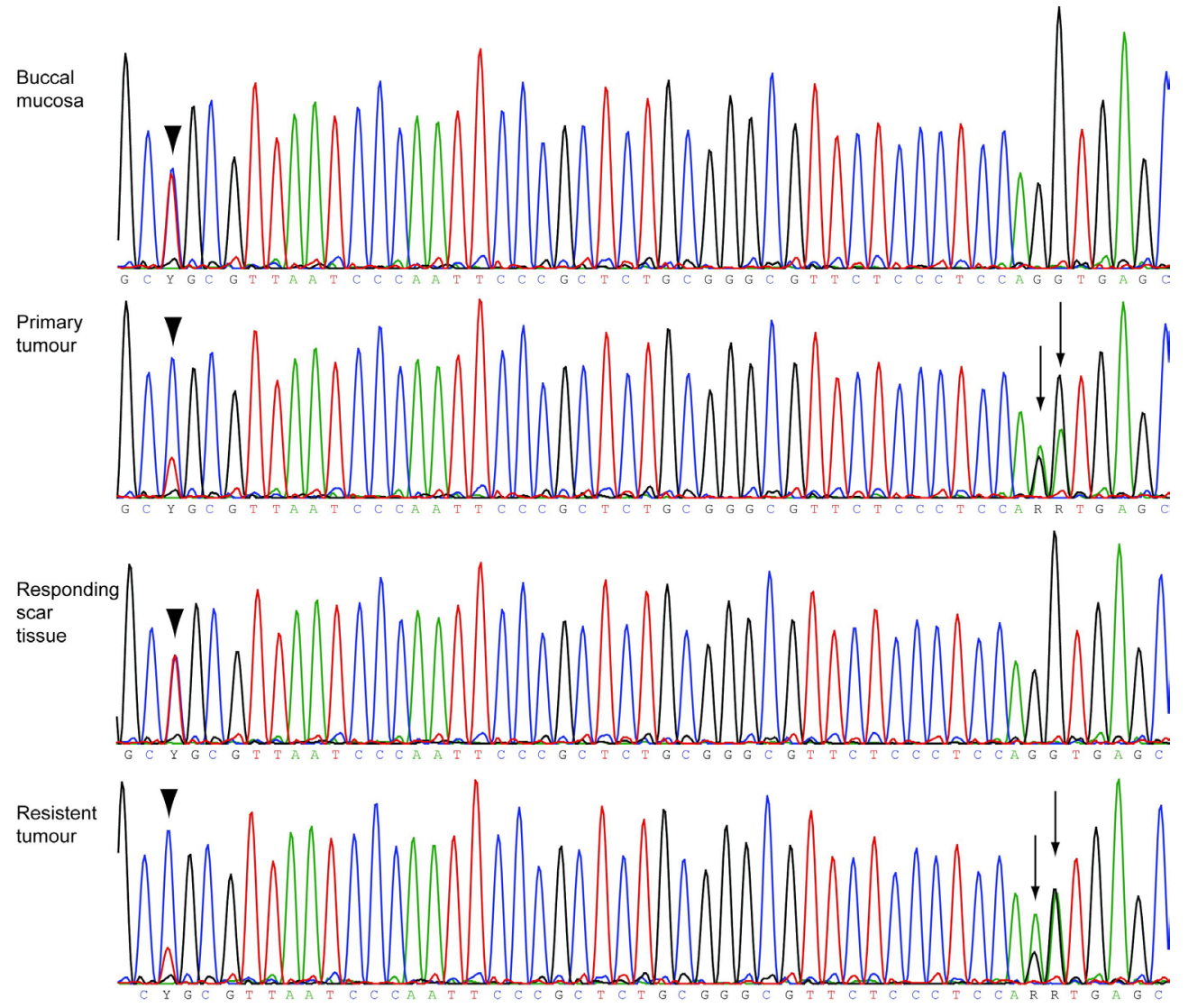

Figure 3. Genomic analysis of PTCH1 coding exons.

The top panel confirmed the absence of a germline mutation in buccal swab. The primary tumor and resistant tumor, panel 2 and 4 respectively both harbored the same mutation and skewed nucleotide distribution. In the scar tissue after vismodegib treatment no PTCH1 mutation could be identified (panel 3). 

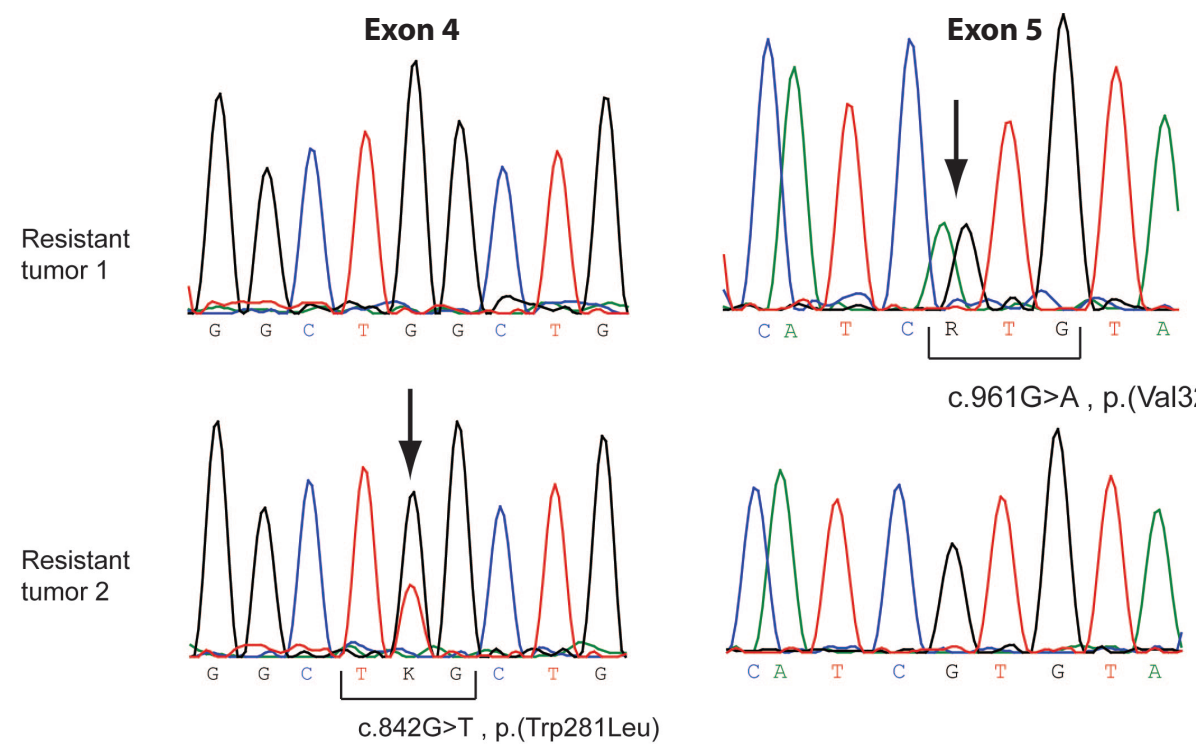

Figure 4. Sequence traces from SMO exon 4 and 5 of resistant tumor tissue.

Top panels represent resistant tumor 1 and lower panels resistant tumor 2. Arrows indicate the mutations in either exon 4 (left panels) for resistant tumor 2 or exon 5 (right panels) for resistant tumor 1 . If the mutation is present in one tumor, it is absent from the other and vice versa. Normal tissue, primary tumor and responding tumor tissue display similar wild type sequence (not shown). 


\section{Discussion}

We describe a patient with a laBCC, who developed resistance to vismodegib after an initial complete clinical response, so-called acquired resistance. Molecular profiling of the primary $\mathrm{BCC}$ revealed a novel somatic mutation in PTCH1. In addition, there was evidence for $\mathrm{LOH}$. The same PTCH1 mutation was present in the recurrent tumor nodules, strongly suggesting that despite a clinical complete response, cells from the original tumor had survived. The mutation could not be detected in the responding tissue, which is consistent with the absence of BCC on histopathology. In the recurrent BCC we detected two different heterozygous missense SMO mutations (codon 281 and codon 321) that have never been reported before in vismodegib resistant $\mathrm{BCC}$. This precludes a clonal origin of the resistant $\mathrm{BCC}$ nests supporting tumor heterogeneity, even though they arose from the presumably clonal primary tumor.

One of the most common mechanisms of resistance to targeted drugs involves secondary genetic alterations in the drug target, disrupting drug binding. There is evidence that these kinds of mutations are already present at a low level before treatment and undergo positive selection during treatment. ${ }^{18}$ Acquired mutations of SMO have already been observed as a cause of secondary tumor growth during treatment with vismodegib in medulloblastoma and BCC. ${ }^{10,14,15}$ In fact, SMO codon 321 was already described by Dijkgraaf et al. as a possible site critical for vismodegib binding. ${ }^{14}$ Codon 281 has never been related to vismodegib resistance and we suggest that our findings extend the SMO domains important for vismodegib binding beyond transmembrane domains (TM) 3, TM5, TM6, TM7 and the third extracellular loop domain including adjacent amino acids (Fig 3 in the Supplementary Appendix). Since the mutations we found were only present in the newly developed tumor nodules, a causal relationship with the development of secondary resistance is very likely. We cannot exclude that there are even more SMO mutations underlying tumor regrowth in this case, because not all new tumor nodules were biopsied.

Preliminary data from the largest study to the efficacy and safety of vismodegib showed complete (17.5\%) and partial response (39.8\%) in 251 assessable advanced BCCs (aBCCs). ${ }^{19}$ Thus resistance to the drug is actually quite common and insight in the mechanism of resistance is important to develop alternative treatment strategies for this group of patients. Possible approaches are combination therapy with different small molecule inhibitors, sequential or rotational therapy with non-treatment periods or alternating different small molecule inhibitors. Advantages of combination therapy in systemic treatment of laBCC or $\mathrm{mBCC}$ can be synergistic therapeutic effects, lower dosage application and, possibly, prevention of (acquired) resistance. ${ }^{20}$ The concept of rotational therapy is currently being investigated in basal cell carcinoma (NCT01815840, clinicaltrials.gov) and in metastatic renal cell carcinoma (NCT01408004, clinicaltrials.gov). A drug holiday or altered dosing can also prevent the emergence of lethal drug resistance as was found in melanoma cell lines treated with vemurafenib. ${ }^{21}$ For combination of sequential therapy, several compounds with a different mode of action than vismodegib are available. Dijkgraaf et al. already found that SMO-antagonist compound 5, from the bis-amide class, was 
effective in the inhibition of vismodegib-resistant mutant SMO in murine allograft models. ${ }^{14}$ Itraconazole, an FDA-approved antifungal agent, inhibits SHH signaling by targeting SMO at a different site from cyclopamine and vismodegib, and offers an alternative systemic treatment of $\mathrm{BCC} .{ }^{22} \mathrm{GLI}$-antagonists target the $\mathrm{SHH}$ pathway downstream of $\mathrm{SMO}$ and may be effective in vismodegib resistant BCC as well. Arsenic Trioxide (ATO) is such a GLI antagonist and already FDA-approved. ATO effectively targets GLI downstream of SMO by blocking accumulation of GLI in the primary cilia and is currently used in treatment of acute promyelocytic leukemia. ${ }^{22}$ The combination of itraconazole and ATO in in vitro and in vivo medulloblastoma murine models was superior to either agent alone. ${ }^{22}$

The phosphatidylinositol 3-kinase / v-akt murine thymoma viral oncogene homologue (PI3K /AKT) pathway is activated in many human cancers, including vismodegib resistant BCC. ${ }^{10}$ ${ }^{23,24}$ Simultaneous administration of SMO antagonists and the PI3K inhibitor NVP-BKM120 delayed the onset of resistance in in vivoand ex vivo medulloblastoma murine models. ${ }^{25} \mathrm{Hence}$, pharmacological inhibition ofbothSHHandPI3KsignalingintumorsresistantforSMOantagonists might be useful and combined therapy should be considered in vismodegib resistant BCC.

It is important to be aware of the possible rapid occurrence of resistance to vismodegib, apparently caused by different acquired mutations. Maybe in the near future, mutational analysis on pre-treatment and recurrent tumor tissue will help to anticipate on the type of resistance, in order to proactively alter treatment. Treatment schedules and combination therapies can then be customized on both patient and tumor characteristics.

In this paper we described a patient with laBCC, in whom two different acquired SMO-mutations underlay the development of resistance to vismodegib, supporting heterogeneity of vismodegib resistant $\mathrm{BCC}$. To our knowledge, to date this phenomenon has not been reported before. 


\section{References}

1. Bath-Hextall FJ, Perkins W, Bong J, Williams HC. Interventions for basal cell carcinoma of the skin. Cochrane database of systematic reviews (Online) 2007:CD003412.

2. Mosterd K, Arits AH, Thissen MR, Kelleners-Smeets NW. Histology-based treatment of basal cell carcinoma. Acta dermato-venereologica 2009;89:454-8.

3. Walling HW, Fosko SW, Geraminejad PA, Whitaker DC, Arpey CJ. Aggressive basal cell carcinoma: presentation, pathogenesis, and management. Cancer metastasis reviews 2004;23:389-402.

4. Moeholt K, Aagaard H, Pfeiffer P, Hansen O. Platinum-based cytotoxic therapy in basal cell carcinoma--a review of the literature. Acta oncologica 1996;35:677-82.

5. Epstein EH. Basal cell carcinomas: attack of the hedgehog. Nature reviews Cancer 2008;8:743-54.

6. Rohatgi R, Scott MP. Patching the gaps in Hedgehog signalling. Nature cell biology 2007;9:1005-9.

7. Axelson M, Liu K, Jiang X, et al. U.S. Food and Drug Administration approval: vismodegib for recurrent, locally advanced, or metastatic basal cell carcinoma. Clinical cancer research : an official journal of the American Association for Cancer Research 2013;19:2289-93.

8. Sekulic A, Migden MR, Oro AE, et al. Efficacy and safety of vismodegib in advanced basal-cell carcinoma. The New England journal of medicine 2012;366:2171-9.

9. Erivedge SmPC, June 2014.

10. Chang AL, Atwood SX, Tartar DM, Oro AE. Surgical excision after neoadjuvant therapy with vismodegib for a locally advanced basal cell carcinoma and resistant basal carcinomas in Gorlin syndrome. JAMA dermatology 2013;149:639-41.

11. Chang AL, Oro AE. Initial assessment of tumor regrowth after vismodegib in advanced Basal cell carcinoma. Archives of dermatology 2012;148:1324-5.

12. Fecher LA. Systemic therapy for inoperable and metastatic basal cell cancer. Current treatment options in oncology 2013;14:237-48.

13. Madan V, Lear JT, Szeimies RM. Non-melanoma skin cancer. Lancet 2010;375:673-85.

14. Dijkgraaf GJ, Alicke B, Weinmann L, et al. Small molecule inhibition of GDC-0449 refractory smoothened mutants and downstream mechanisms of drug resistance. Cancer research 2011;71:435-44.

15. Yauch RL, Dijkgraaf GJ, Alicke B, et al. Smoothened mutation confers resistance to a Hedgehog pathway inhibitor in medulloblastoma. Science 2009;326:572-4.

16. Metcalfe C, de Sauvage FJ. Hedgehog fights back: mechanisms of acquired resistance against Smoothened antagonists. Cancer research 2011;71:5057-61.

17. Atwood SX, Chang AL, Oro AE. Hedgehog pathway inhibition and the race against tumor evolution. The Journal of cell biology 2012;199:193-7.

18. Garraway LA, Janne PA. Circumventing cancer drug resistance in the era of personalized medicine. Cancer discovery 2012;2:214-26.

19. Rainer Kunstfeld J-JG, Brigitte Dréno, Thomas Jouary, Laurent Mortier, Paolo A. Ascierto, Axel, Hauschild JH, Lada Mitchell, Michal Starnawski, , Basset-Seguin N. Vismodegib, a Hedgehog Pathway Inhibitor, in Advanced Basal Cell Carcinoma: STEVIE Study Interim Analysis in 300 Patients. European Academy of Dermatology and Venereology. Istanbul2013.

20. Flaherty KT, Infante JR, Daud A, et al. Combined BRAF and MEK inhibition in melanoma with BRAF V600 mutations. The New England journal of medicine 2012;367:1694-703.

21. Das Thakur M, Salangsang F, Landman AS, et al. Modelling vemurafenib resistance in melanoma reveals a strategy to forestall drug resistance. Nature 2013;494:251-5.

22. Kim J, Aftab BT, Tang JY, et al. Itraconazole and arsenic trioxide inhibit Hedgehog pathway activation and tumor growth associated with acquired resistance to smoothened antagonists. Cancer cell 2013;23:23-34. 
Acquired resistance to Vismodegib due to smoothened mutations in treatment of locally advanced BCC | 171

23. Hafner C, Landthaler M, Vogt T. Activation of the PI3K/AKT signalling pathway in non-melanoma skin cancer is not mediated by oncogenic PIK3CA and AKT1 hotspot mutations. Experimental dermatology 2010;19:e222-7.

24. Jee SH, Chu CY, Chiu HC, et al. Interleukin-6 induced basic fibroblast growth factor-dependent angiogenesis in basal cell carcinoma cell line via JAK/STAT3 and PI3-kinase/Akt pathways. The Journal of investigative dermatology 2004;123:1169-75.

25. Buonamici S, Williams J, Morrissey M, et al. Interfering with resistance to smoothened antagonists by inhibition of the PI3K pathway in medulloblastoma. Science translational medicine 2010;2:51ra70. 

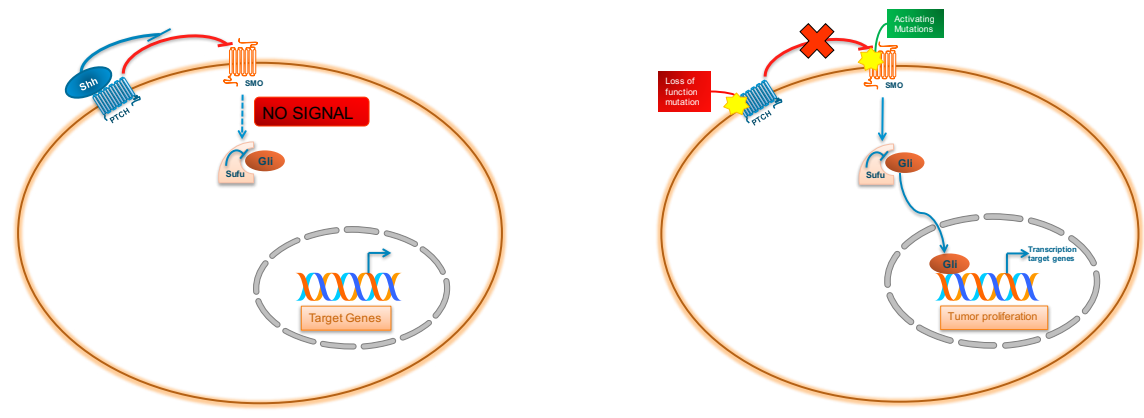

\section{Supplementary Figure 1: Schematic presentation of the Hh signaling pathway.}

A. Hh signaling during normal development. The 12-transmembrane protein Patched 1 (PTCH1) inhibits Smoothened (SMO), thereby preventing translocation of Gli to the nucleus and suppressing Hh signaling. B. Hh signaling in basal cell carcinoma. Inactivating mutations in PTCH1, preventing inhibition of SMO, or activating mutations, in $\mathrm{SMO}$ lead to upregulation of $\mathrm{Hh}$ signaling, resulting in transcription of target genes and tumor proliferation.

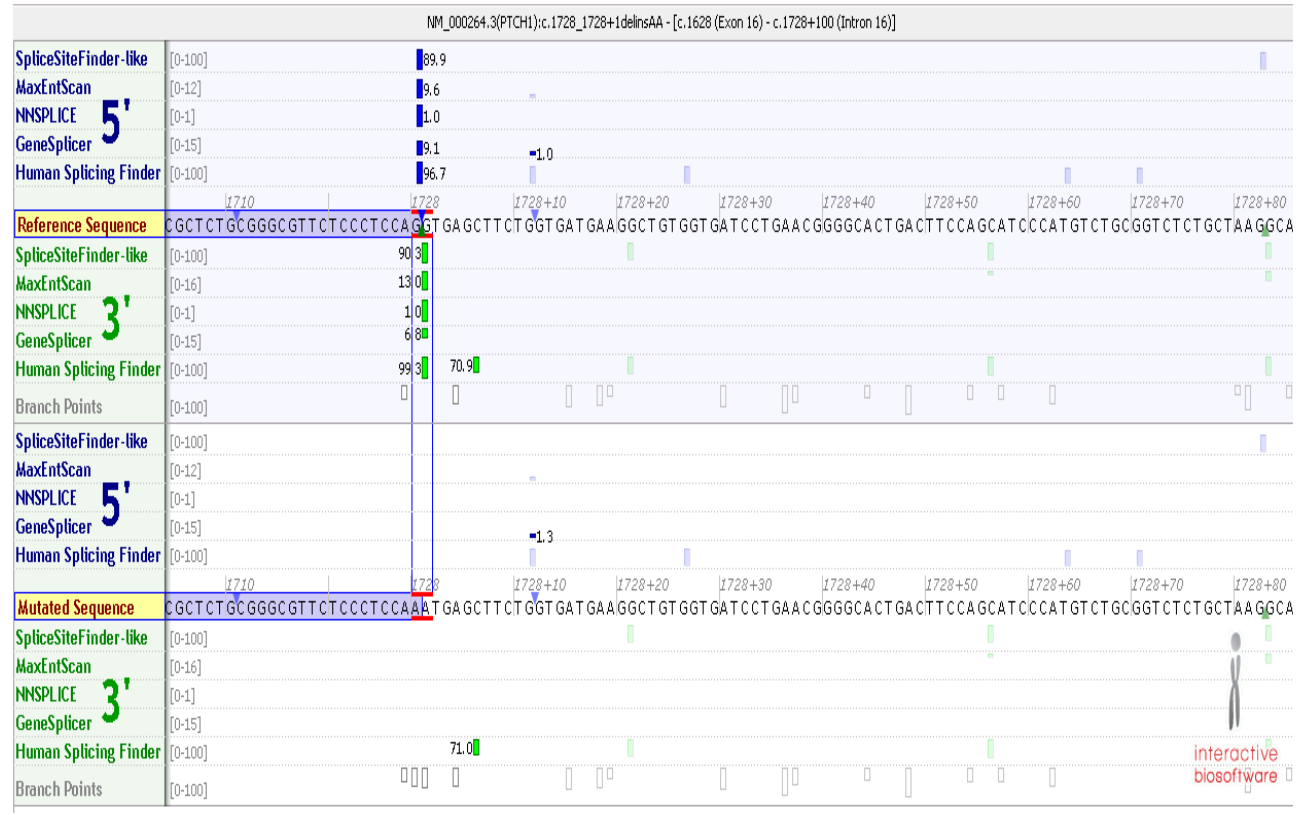

\section{Supplementary Figure 2: Alamut output for PTCH1.}

Analyses of the heterozygous c.1728_1728+1delinsAA PTCH1 mutation using Alamut version 2.3, which included 5 different splice site prediction program. Mutated sequences ends in a STOP codon, confirming the pathogenicity of the mutation. 


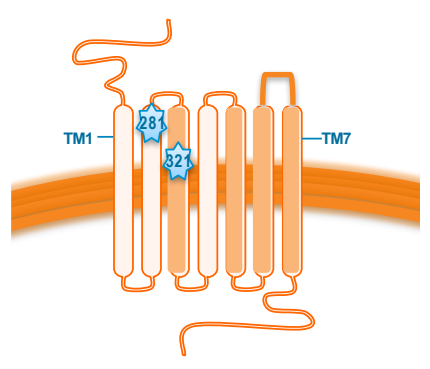

\title{
Supplementary Figure 3: Schematic representation of SMO.
}

\begin{abstract}
Schematic representation of the SMO protein with the 7 transmembrane (tm) helices. Tm 3, 5, 6 and 7 are thought to be most important in vismodegib binding. Shown are codon 281 and 321 , which are the mutation sites in our patient.
\end{abstract}

\section{Supplementary material: Methods}

Our patient provided written informed consent for the use of both clinical pictures and tissue specimens for research purposes. Biopsies were taken from tumor tissue before treatment with vismodegib. After the occurrence of resistance, biopsies were taken from clinically responding and non-responding skin. Each (tumor) tissue sample was coated with Tissue-Tek® O.C.T. (Sakura) and immediately frozen and stored at $-80^{\circ} \mathrm{C}$. From all tissue samples a $4 \mu \mathrm{m}$ section was stained with haematoxylin-eosin (HE) as part of routine diagnostic procedure. All HE sections were reviewed by a dermatopathologist to confirm diagnosis and histological subtype.

Genomic DNA was extracted by using NucleoSpin® Tissue XS (Machery-Nagel), following the manufacturer's directions. We extracted DNA from 10 sections of $10 \mu$ m of each tissue specimen. Mutation analysis of the PTCH1 gene and SMO gene was performed to unveil the primary mutation leading to development of $\mathrm{BCC}$. $\mathrm{LOH}$ of the $\mathrm{PTCH} 1$ gene was confirmed with allele specific sequencing by using cloning and restriction fragment analysis showing that the C-allele containing clone also contains the deletion/insertion mutation and only $15 \%$ of the clones ( 5 clones out of 33 clones) contain the T-allele. Primer sequences are available upon request. 



\title{
Chapter 5.2
}

Hyperkeratosis, epidermal cyst formation and tumor resistance in a patient treated with vismodegib for locally advanced basal cell carcinoma

\author{
Reinders MG*, Brinkhuizen T*, Soetekouw PM, Kelleners-Smeets \\ NWJ, Abdul Hamid MA, Mosterd K.
}

*Both authors contributed equally to this paper and should be considered as first authors.

Acta Dermato Venereologica 2015 Apr 28;95(5):618-619. 


\section{Introduction}

Vismodegib, a Sonic Hedgehog (SHH) pathway inhibitor, was approved by the US Food and Drug Administration (FDA) in 2012 for the treatment of locally advanced (la) and metastatic ( $\mathrm{m}$ ) basal cell carcinoma (BCC). Interim results of the largest clinical study of vismodegib have shown complete (17.5\%) or partial (39.8\%) response, and stable (39.0\%) or progressive (2.8\%) disease in 251 cases of laBCC and mBCC. ${ }^{1}$ Formation of squamous cell carcinoma (SCC) within the tumor area, as well as occurrence of SCC at other body sites, has been reported in patients treated with vismodegib. ${ }^{2-6}$ To our knowledge this is the first report of formation of epidermal cysts and hyperkeratosis within the tumor area during treatment with vismodegib.

\section{CASE REPORT}

A 52-year-old man was referred to our hospital with a recurrent BCC that was treated with cryotherapy 15 years previously, followed by multiple incomplete excisions. An area of scar tissue and minimal hyperkeratosis was visible on the nose, extending to his right cheek and upper lip (Fig. 1A). Several biopsies were taken, and showed infiltrative BCC invading the deep dermis; there was subtle hyperkeratosis, but no evidence of SCC or BCC with squamous differentiation (Fig. 2A).
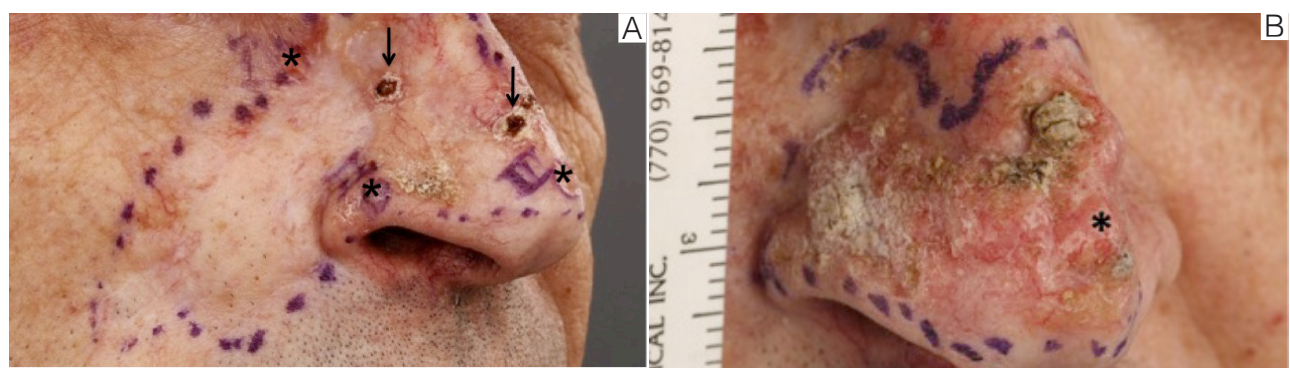

Figure 1.

A. Clinical picture before treatment with vismodegib. Biopsy sites $\left(^{*}\right)$ were positive for infiltrative basal cell carcinoma (BCC). Crusts from earlier biopsy sites $(\downarrow)$, also positive for BCC. B. Clinical picture with extensive hyperkeratosis and comedo-like lesions after 7 weeks' treatment with vismodegib. Biopsy site $\left(^{*}\right)$.

To avoid mutilating surgery, the patient was enrolled in a clinical trial with oral vismodegib 150 mg daily. ${ }^{7}$ This trial was approved by the ethics and scientific committee of the Maastricht University Medical Centre. After 7 weeks of treatment, hyperkeratosis increased and comedo-like lesions developed on the nose (Fig. 1B). Sequential skin biopsies showed epidermal cysts, but no residual BCC was found. Because of the impressive hyperkeratosis, we feared for progression into SCC despite the absence of malignancy in repeated biopsies, taking the possibility of a sampling error into account. Therefore, we performed Mohs' micrographic surgery 5 months after initiation of vismodegib therapy, which was continued until the day before surgery. Five stages of Mohs' surgery with 19 frozen sections, including nose amputation, were necessary to achieve clear margins. Histological examination 
showed residual infiltrative BCC, accompanied by epidermal cyst formation and some hyperkeratosis, but no evidence of SCC (Fig. 2B). Immunohistochemical analysis confirmed residual BCC cells by positive staining for Ber-Ep4, whereas epidermal cysts stained negative.

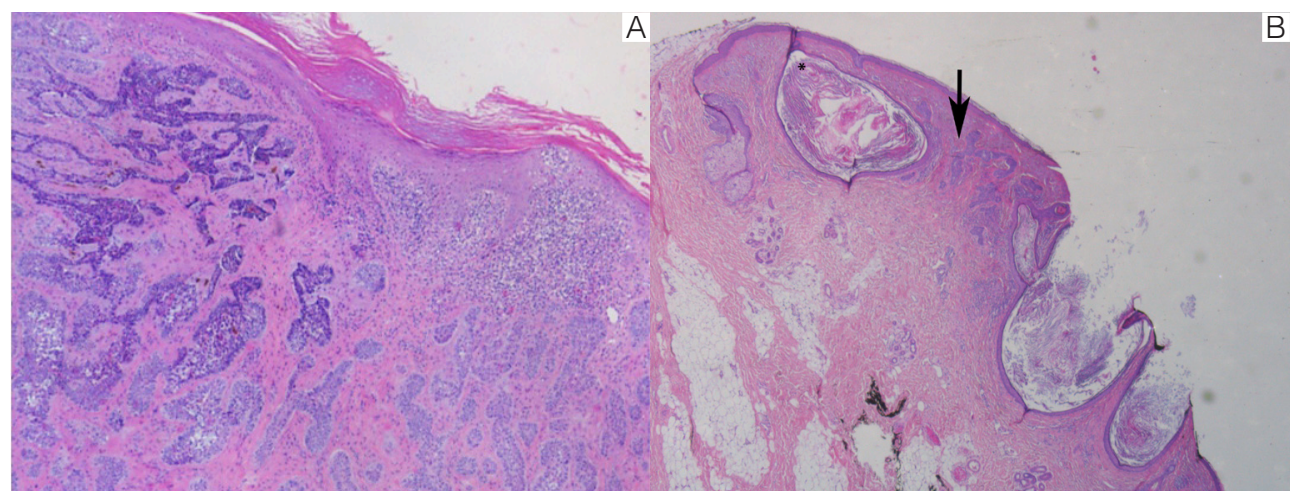

Figure 2. Histological examination.

A. Skin biopsy before treatment with vismodegib, showing infiltrative basal cell carcinoma (BCC). B. Skin excision specimen with epidermal cyst $\left(^{*}\right)$ formation and infiltrative BCC $(\downarrow)$. Haematoxylin-eosin stain $(20 \times)$.

\section{Discussion}

Pseudocyst-like structures as a result of tumor regression during the use of vismodegib have been reported previously; however, they were characterized by a different histology with so-called pseudocystic areas consisting of extensive fibrosis and some residual BCC cells but without keratin formation. ${ }^{8}$ Formation of SCC within the initial BCC has been reported in several patients treated with vismodegib as well as development of SCC and keratoacanthoma on other body sites. ${ }^{2-6}$ It is therefore important to be aware of the possibility of occurrence of SCC during vismodegib treatment and to perform sequential biopsies from suspicious areas. Tumor heterogeneity may underlie the emergence of SCC within the tumor area. Squamous differentiation is common in BCC and a subset of malignant squamous cells could become predominant when the BCC is treated with vismodegib. However, this does not explain the occurrence of squamous neoplasms on other sites of the body or, as in our case, the development of benign squamous neoplasia. Furthermore, the epithelial cell marker Ber-EP4 negative immunohistochemical staining of the epidermal cysts in our patient speaks against the survival of cells from the original tumor, as BCC and basosquamous carcinoma stain Ber-EP4 positive. A possible explanation for the emergence of the benign squamous neoplasms could be an effect of vismodegib on keratinocyte differentiation. In essentially all BCC, loss of function mutations in the tumor suppressor gene Patched1 $(\mathrm{PTCH} 1)$ prevent binding of $\mathrm{SHH}$ to the transmembrane protein $\mathrm{PTCH}$, resulting in activation of the $\mathrm{SHH}$ signaling pathway and eventually tumor formation. The SHH pathway seems to be important in keratinocyte differentiation and proliferation, as Indian hedgehog $(\mathrm{IHH})$, another member of the $\mathrm{HH}$ ligand family, plays an important role 
here, and loss of $\mathrm{IHH}$ promotes the progression of benign papillomas to SCC. ${ }^{9}$ Vismodegib treatment could mimic loss of $\mathrm{IHH}$, as both reduce $\mathrm{SHH}$ pathway activation. Therefore, an effect of vismodegib on keratinocyte differentiation seems plausible. Functional analysis might clarify the exact effect of vismodegib on keratinocyte differentiation and proliferation. 


\section{References}

1. Grob JJ KR, Dreno B, Jouary T, Mortier L, Basset-Seguin N, Ascierto PA, Hansson J, Mitchell L, Starnawski M, Hauschild A. Vismodegib, a Hedgehog pathway inhibitor (HPI), in advanced basal cell carcinoma (aBCC): STEVIE study interim analysis in 300 patients. Poster discussion meeting. American Society of Clinical Oncology, 2013.2013.

2. Iarrobino A, Messina JL, Kudchadkar R, Sondak VK. Emergence of a squamous cell carcinoma phenotype following treatment of metastatic basal cell carcinoma with vismodegib. Journal of the American Academy of Dermatology 2013;69:e33-4.

3. Saintes C, Saint-Jean M, Brocard A, et al. Development of squamous cell carcinoma into basal cell carcinoma under treatment with Vismodegib. Journal of the European Academy of Dermatology and Venereology : JEADV 2015;29:1006-9.

4. Zhu K, Zhang W, Wu A, Zhang X. A comparative study of two wayfinding aids for simulated driving tasks single-scale and dual-scale GPS aids. Behaviour \& Information Technology 2014;33:361-71.

5. Orouji A, Goerdt S, Utikal J, Leverkus M. Multiple highly and moderately differentiated squamous cell carcinomas of the skin during vismodegib treatment of inoperable basal cell carcinoma. The British journal of dermatology 2014;171:431-3.

6. Aasi S, Silkiss R, Tang JY, et al. New onset of keratoacanthomas after vismodegib treatment for locally advanced basal cell carcinomas: a report of 2 cases. JAMA dermatology 2013;149:242-3.

7. STEVIE: A study of vismodegib in patients with locally advanced or metastatic basal cell carcinoma. 2011 May 30 ed. ClinicalTrials.gov. : Bethesda (MD): National Library of Medicine (US).

8. Maier T, Kulichova D, Ruzicka T, Berking C. Noninvasive monitoring of basal cell carcinomas treated with systemic hedgehog inhibitors: pseudocysts as a sign of tumor regression. Journal of the American Academy of Dermatology 2014;71:725-30.

9. Kakanj P, Reuter K, Sequaris G, et al. Indian hedgehog controls proliferation and differentiation in skin tumorigenesis and protects against malignant progression. Cell Rep 2013;4:340-51. 



\section{Chapter 6}

Discussion \& valorization 
BCC is the most common cancer worldwide and its incidence is still rising, by $3-10 \%$ annually. However, as there are few adequate skin cancer registries, the actual increase might be even higher. Although BCCs rarely metastasize and mortality is extremely low, they can cause significant morbidity due to locally aggressive behavior. Half of all BCC arise primarily on the face and (bald) scalp. Surgical excision, which is the current gold standard of treatment, has disadvantages, such as the risk of bleeding, infection, impaired healing and scarring, which can be disfiguring and cause patients to experience a significantly decreased quality of life.

The high incidence of BCC combined with the associated high costs of treatment and the attendant workload is causing a significant and mounting impact on the health-care system. Consequently, BCC is an important public health problem. For that reason, there is an urgent medical and societal need for a patient friendly and cheap (targeted) non-invasive treatment, and preferably one that can be performed by patients themselves. To develop such a treatment, a profound understanding of BCC pathogenesis is essential. The research described in this thesis aims to profile genetic and epigenetic alterations in both non-aggressive and locally advanced BCC in order to enhance our understanding of BCC pathogenesis. This will be described in part one. Part two discusses new topical and systemic drugs in the treatment of BCCs.

\section{Part one: pathogenesis of BCC}

\section{Epigenetics and more in BCC}

Epigenetics encompasses heritable changes in gene expression without changes in the DNA sequence. These changes can be present at the DNA (DNA methylation), RNA (RNA interference by non-coding RNAs), and protein (histone modifications, polycomb group proteins, and chromatin remodeling) level. The continuous interplay of these epigenetic regulations determines the organism's phenotype, which is referred to as the epigenome. ${ }^{1,2}$ The studies presented in this thesis focus on DNA-methylation, one of the most important and well-studied epigenetic alterations. DNA methylation consists of adding a methyl group to cytosine nucleotides, primarily when they are followed by a guanine, the so-called CpG dinucleotide. Aberrant DNA methylation patterns are seen in many cancers (o.a. colon, breast, kidney and prostate cancer $)^{1}$ and other human diseases, such as autoimmune and neurological disorders. ${ }^{3}$ In BCCs, however, only a few studies have so far looked at DNA methylation. Also, these studies focused on predefined tumor suppressor genes and did not provide evidence that any observed changes in methylation patterns were relevant for BCC carcinogenesis. ${ }^{4-9}$ Additional evidence shows that ultraviolet (UV) radiation A and UVB may alter epigenetic modifications ${ }^{10}$ and $U V$-signature mutations seem to preferentially occur at methylated C-phosphate-G ( ${ }^{\mathrm{m} C p G}$ ) dinucleotides. ${ }^{11}$ As BCCs mainly occur at sun-exposed areas of the skin ${ }^{12}$, they might be expected to show extensive methylation changes compared to non-diseased skin.

In a first exploration of promoter hypermethylation in BCCs, we assessed the promoter CpG island methylation status of nine candidate tumor suppressor genes (TSG). CpG islands 
are regions with a higher frequency of $\mathrm{CpG}$ dinucleotides that are mostly found around the promoter region of the gene. ${ }^{13}$ As described in chapter 2.1, nested methylation specific polymerase chain reaction (MSP) was performed on bisulfite-modified genomic DNA of BCCs and healthy skin with primers specific for methylated DNA, respectively unmethylated DNA. Significantly more DNA methylation in BCCs was found in the gene promoters of SHH, APC and SFRP5, all key regulators of the SHH and WNT signaling pathways, and of RASSF1A. RASSF1A is a well-known tumor suppressor of which epigenetic inactivation is previously described in $\mathrm{BCCs}^{9}$, but also in malignant melanoma ${ }^{14}$ and Merkel cell carcinoma. ${ }^{15}$ Interestingly, healthy sun-exposed (SE) skin samples showed methylation patterns similar to sun-protected (SP) skin, suggesting that the methylation we observed is more tumor-specific than locus or patient specific, a finding that is in line with literature to date. ${ }^{16}$ It is of interest that methylation patterns in sun-exposed skin did not resemble those in BCC. Our findings therefore do not support a contribution of UVB exposure to the observed promoter hypermethylation of SHH, APC, SFRP5 and RASSF1A. However, since the epigenetic changes that we found parallel genetic changes driving $B C C$ growth, it seems likely that gene silencing by promoter hypermethylation is relevant to BCCs.

In this study we used nested MSP because it is highly sensitive and can detect 1 methylated allele in >50,000 unmethylated alleles. This makes it exquisitely suitable for methylation analysis of archived formalin-fixed paraffin-embedded (FFPE) DNA, as this generally yields small amounts of poor quality DNA. ${ }^{17}$ Since biopsy or excision tissue samples taken during regular care are commonly FFPE and stored in the archives of the pathology department, there is wealth of tissue available for research. On the down side, (nested) MSP is a 'yes or no' approach that does not provide quantitative results. Also, it can only be used to screen pre-defined candidate genes. Fortunately, novel techniques such as comprehensive probe-based, bisulfite whole- genome sequencing, and enrichment-based technologies ${ }^{18-20}$ allow unbiased genomewide analysis of DNA methylation (the "methylome"). This is a major advantage compared to locus-specific, low-throughput approaches that are typically PCR based. However, the latter are still used for validation purposes. ${ }^{19}$

Chapter 2.2 describes the first analysis of the BCC methylome by genome-wide ${ }^{m} C p G$ DNA enrichment sequencing (MethylCap-seq). ${ }^{21}$ MethylCap-seq is based on capture of methylated DNA on a genome-wide scale with the methyl-DNA binding domain (MBD) domain of the protein MeCP2. As only methylated DNA is subsequently sequenced, the complexity of the analysis is reduced and high sequence coverage can be obtained. ${ }^{22}$ MethylCap-seq is a robust, highly reproducible approach, especially for larger sample cohorts. ${ }^{22}$

We identified 32979 methylated regions in 22121 genes, 13 of which were differentially methylated in sporadic BCCs compared to healthy skin, with a False Discovery Rate (FDR) of $<0.1$ (chapter 2.2). As methylation can regulate gene expression levels ${ }^{23}$, we performed rRNA depleted, strand specific, total RNA sequencing (RNA-seq henceforth) and correlated these data to the methylome. This correlation provides information on the functional methylome: i.e. those methylation events that affect gene expression. Of note, there was little overlap 
between the top-ranked differentially methylated loci and differentially expressed genes $(F D R<0.1)$, even when preselecting for genes that might be differentially expressed in BCC. At present, we cannot explain this finding. The full spectrum of functions that DNA-methylation has remains to be delineated, as is its full impact on RNA expression levels. IFI30 was one of the hits that was both differentially methylated and differentially expressed, a finding that points to the importance of immune signaling in BCC. IFI30 is known to play a critical immunoregulatory role in melanoma by mediating antitumor immunity via CD4+ $\mathrm{T}$-cells. ${ }^{24}$

We compared the results of our first analysis in BCC with those obtained from methylCap-seq and RNA analysis of a facial laBCC (chapter 2.3). We identified considerable agreements and differences between the non-aggressive and aggressive BCCs. The most striking difference was a dramatic increase in expression of matrix metalloproteinase 13 (MMP13) in the laBCC, which could explain the more aggressive behavior of this tumor. ${ }^{25}$

Additional pathway analysis of the RNA-seq data was performed with SPIA software ${ }^{26}$, showing that pathways activated in BCC are all involved in immune signaling: cytokine-cytokine receptor interaction signaling (KEGG hsa04060), chemokine signaling (Chemokine) (KEGG hsa04062) and Toll-like receptor signaling (KEGG hsa04620). Pathway enrichment analysis of the differentially methylated genes also revealed cytokine-cytokine receptor interaction as the most significant pathway. The importance of immune signaling in BCCs is further supported by results from our independent clinical trial, described in chapter 4.1. Previous work has also found increased expression of innate immune genes in squamous cell carcinomas (SCCs) and in BCCs. ${ }^{27}$

Apparently, $\mathrm{SHH}$ signaling might not be the sole driver in sporadic BCCs. In fact, BCC pathogenesis seems to be rather complex, and this is further confirmed by the whole-exome sequencing of BCCs described by Jayaraman et.al. ${ }^{28}$ Surprisingly, although BCC can be considered as the least malignant human cancer, they harbor the largest mutational burden compared to any other known human malignancy. The vast majority of mutations $(75.7 \%)$ showed a clear UV signature. However, Jayaraman et.al. could not identify any novel genes as drivers in BCC. The authors hypothesized that the resulting high level of mutated proteins might increase the hosts' immune response, resulting in a less aggressive phenotype. ${ }^{28}$ It is of interest that the incidence of nonmelanoma skin cancer (NMSC) in immunosuppressed patients, such as organ transplant recipients (OTR), is up to 250-fold higher than in the general population. For common cancers like gastrointestinal, lung, breast, bladder, prostate, and gynecological, this risk is only two to five-fold higher. ${ }^{29,30}$ In OTR, SCCs occur 4-10 times more often than BCCs and tend to behave in a more aggressive manner with increased metastatic potential, and higher recurrence rates compared to SCC in non-immunocompromised people. ${ }^{30,31}$ This increased risk of SCCs and also BCCs may be due to the higher rates of human papillomavirus (HPV) infection in immunosuppressed populations, however the most convincing evidence is available for the link between HPV and SCC. An etiologic association between HPV and BCC remains to be elucidated. ${ }^{32}$ In addition to viral oncogenic infections, decreased immune-surveillance and the direct effect of the immunosuppressive medications 
used by OTR causes them to be more vulnerable to NMSC development. ${ }^{32}$ The influence of an immune response in BCC development was previously reported and shows that transplantation of human BCC biopsies is only successful in mice deficient of $\mathrm{T}, \mathrm{B}$ and natural killer (NK) cells. ${ }^{33}$ However, BCC nest are frequently, but not always, surrounded by an infiltrate consisting of $T$ cells and only very few B lymphocytes and NK cells that do generally not invade the tumor nests. ${ }^{34}$ Although complete tumor eradication is rare ${ }^{30}$, higher number of $\mathrm{T}$ cells is seen in actively regressing BCCs. ${ }^{34}$ Generally, BCCs express no or low levels of $\mathrm{MHC}$ class I (MHC-I) molecules and as a consequence there is an absence of infiltrating CD8 ${ }^{+}$ effector T cells. Following treatment with topical imiquimod, a Toll-like receptor-7 antagonist, significantly higher expression levels of $\mathrm{MHC}-\mathrm{I}$ accompanied by an increase of peritumoral $\mathrm{CD}^{+} \mathrm{T}$ cells were seen. ${ }^{30}$ This pro-inflammatory Th1 initiated anti-tumor immune response has also been implicated in the well-established spontaneous regression of BCC after a biopsy. ${ }^{35}$ Also, A Th2 anti-inflammatory response has been described in BCCs, which suggests a dynamic state in where pro- and anti-inflammatory immune response coexist and compete. ${ }^{36}$ The exact mechanisms underlying an effective tumor specific immune response is unclear as there are still many missing links. In our study described in chapter $\mathbf{4 . 1}$, an extensive infiltrate was seen in the BCCs treated with topical diclofenac, in the majority of the cases even resulting in complete histological tumor regression. The nature of this immuneresponse will be analyzed in a future study in an attempt to unravel the role of the BCC immuneresponse.

\section{DNA methylation in BCC and the future}

As epigenetic alterations are potentially reversible, drugs have been developed to inhibit enzymes that catalyze epigenetic modifications. In the case of DNA methylation, these are the DNA methyltransferases (DNMTs), consisting of 3 main subtypes. DNMT1 maintains existing methylation during DNA replication, while DNMT3A and DNMT3B are required for de novo methylation. To date, the FDA has approved two demethylating drugs for the treatment of higher-risk myelodysplastic syndrome: 5-azacytidine (5-aza-CR; manufactured by Celgene as Vidaza) and 5-aza-2'-deoxycytidine (5-aza-CdR; manufactured by Eisai as Dacogen). However, these drugs have substantial side effects, which makes them less useful for BCC treatment, as the advantage of being a non-invasive treatment will not outweigh these side effects. ${ }^{37}$ The systemic side effects could be avoided by using the 5-aza's topically. Unfortunately, they are both pharmalogically unstable agents, which impedes their topical formulation.

Whereas DNA methylation changes in cancer constitute a potential target for treatment, they could also be used to develop biomarkers for more accurate detection of cancer, a better insight in tumor prognosis, and even to predict treatment response. ${ }^{2}$ In contrast to genetic biomarkers that detect the numerous different point mutations occurring throughout the length of the gene, DNA hypermethylation consistently affects a small promoter region, facilitating analysis. Also, DNA methylation alterations occur in higher percentages of tumors than genetic variations, resulting in a higher sensitivity. ${ }^{37}$ The utility of biomarkers for BCC treatment can be debated, as these tumors are generally characterized by indolent growth, an excellent prognosis and easy accessibility 
for assessment and treatment. However, given the large number of patients that will develop at least one or more subsequent BCC after the first BCC, a prognostic biomarker that could predict the individual risk of (recurrent) BCC could be extremely useful. It would enable classification of patients as having a low, an intermediate or a high risk of developing BCC. Using this information, follow-up schemes could be tailored to the patient's individual risk, thereby making care for BCC more efficient and cost-effective.

\section{Beyond DNA methylation}

In our work, we focused on DNA (cytosine) methylation, because it is the best-defined epigenetic change in malignancy. Hydroxymethylation of cytosine $(5-\mathrm{hmC})$ is a more recently described modification, that affects the flexibility and stability of DNA differently from methylation. The 5-hmC level is strongly reduced in tumors of the brain, kidney, lungs, skin and liver, from which it was suggested that 5-hmC loss could serve as biomarker for the detection of cancers. However, the exact role of 5 -hmC in the development of cancer remains unknown. ${ }^{38,39}$

Additional epigenetic modifications that have been found to be involved in human cancers include histone modifications, chromatin remodeling and the activity of non-coding RNAs (ncRNAs). ${ }^{3,40}$ These different processes work together to establish and maintain the global and local condensed or decondensed chromatin states that eventually determine gene expression. ${ }^{2}$ DNA is tightly compacted around histones to the nucleosome which is the basic unit of the chromatin. ${ }^{40}$ These histones can be modified post-transcriptionally by (hyper) acetylation, methylation, phosphorylation and ubiquination. Those different processes affect, among others, transcriptional regulation, DNA repair, DNA replication, alternative splicing, and chromosome condensation. ${ }^{3}$ Hyperacetylation of the lysines in histone tails is the best described modification and is facilitated by Histone Acetyltransferases (HATS). This results in a decondensed chromatin confirmation that can be actively transcribed. On the other hand, histone deacetylases (HDACs) restore the condensed state, resulting in gene silencing by preventing binding of transcription factors and RNA polymerases. ${ }^{40}$ In the case of tumor suppressor genes, this means that tumor development is facilitated. Thus, HDAC inhibition could be used to treat cancer. Indeed, Vorinostat (SAHA). a synthetic HDAC inhibitor (HDACi) was approved by the FDA in 2006 for the treatment of cutaneous T-cell lymphoma. ${ }^{41}$ Two more (romidepsin (Istodax) and belinostat (Beleodaq)) have followed in the past few years and many HDACis are in different stages of clinical trials for various hematological and solid tumors. ${ }^{42}$ Apart from the direct effect of HDCAis on acetylation resulting in re-expression of the gene, HDCAis can also target processes involved in tumor progression, cell cycle control, apoptosis, angiogenesis and cell invasion. ${ }^{42}$ Interestingly, HDACis have been shown to suppress $\mathrm{SHH}$ activity through Gli1, Gli2 and Gli3 in vitro. ${ }^{43}$ A newly designed chimeric compound, NL-103, which integrates the functional inhibitory group of the HDACi vorinostat and $\mathrm{SHH}$ inhibitor vismodegib, downregulates the expression of Gli2 in a NIH3T3-12Gli mouse embryo fibroblast cell line. It was concluded that NL103 can effectively inhibit SHH and that dual inhibition of HDACs and SHH signaling could help to overcome vismodegib ${ }^{44}$, a phenomenon that is described in more detail in part two. 
The dynamic process of chromatin remodeling is not only regulated by histone acetylation/ deacetylation, but also through ATP-dependent protein complex formation, histone modification by polycomb proteins, and by interaction with ncRNAs. ${ }^{40}$ ncRNAs are RNA fragments that are not translated into proteins of which several subtypes can be identified such as miRNA, siRNA, piRNA, XiRNA and long ncRNAs. ${ }^{40}$ RNA interference (RNAi) is a part of a small regulatory $R N A$, including siRNA and miRNA, that regulates gene silencing. RNAi based therapies are already being tested in phase 1 studies as treatment in several solid tumors (brain, breast) and considered to be safe in use. These drugs have the benefit of being highly specific and they have only little side effects. ${ }^{45} \mathrm{~A}$ comprehensive description of all possible epigenetic modification processes is beyond the scope of this thesis. But it is evident that the rise in knowledge on epigenetic modifications in human cancers like BCC can yield considerable diagnostic, therapeutic and even predictive opportunities in the (near) future.

\section{mTOR signaling in BCC}

Whereas most research on $\mathrm{BCC}$ has been focused on $\mathrm{SHH}$ and WNT signaling, it is important to think out of the box in order to unravel the pathogenesis of this tumor completely. As an example of such thinking, when looking for therapeutic targets we considered that BCCs are thought to be hair follicle tumors. ${ }^{46}$ The hair follicle is considered as a moderately to severely hypoxic microenvironment, while the dermis is well oxygenated and the epidermis is only modestly hypoxic. ${ }^{47}$ Therefore we hypothesized that hypoxia response pathways could be involved in the pathogenesis of hair follicle tumors such as BCCs. Hypoxia-inducible transcription factor 1 (HIF1) is a primary mediator of hypoxia-induced gene expression in human hair follicles. ${ }^{48} \mathrm{HIF} 1$ transcription can be regulated by mechanistic/mammalian target of rapamycin 1 (mTORC1) via 4E-binding protein 1 (4E-BP1), ribosomal protein S6 kinase-1 (S6K1) and signal transducer and activation of transcription 3 (STAT3). ${ }^{49}$ mTORC1 in turn is stimulated by PI3K-Akt signaling pathways and once activated, it promotes several processes like angiogenesis, cell growth and tumorigenesis. ${ }^{50}$ The importance of mTORC1 in the pathogenesis of different cancer types, including renal carcinoma and colon carcinoma is well recognized and has already resulted in the development of treatments that target mTORC-dependent signaling events. ${ }^{51}$ Interestingly, activated mTORC signaling is also associated with the development of benign skin tumors like angiofibromas in patients with tuberous sclerosis. ${ }^{48}$

If HIF and mTORC1 pathways do contribute to the growth of hair follicle tumors, new opportunities for targeted therapy and diagnostics could emerge. In chapter $\mathbf{3 . 1}$ we provide a systematic analysis of HIF and mTORC1 signaling in BCC and its benign counterpart, trichoepithelioma (TE). TEs strongly resemble BCC both on the macroscopic and microscopic level, but do not exhibit invasive growth. Differentiation between these two hair follicle-derived tumors is important because of their distinct biologic behavior and therapeutic approach. Immunohistochemical analysis of HIF1, mTORC1 and their most important target genes showed that both HIF and mTORC1 signaling seem to be active in BCC as well as in TE. However, there were no appreciable differences between these two tumors with respect to pathway activity. To date, immunohistochemical analyses of HIF, mTORC1 and their tar- 
get genes does not provide a reliable diagnostic tool for the discrimination of BCC and TE.

In conclusion, we and others have shown that sporadic BCCs are tumors driven by a variety of derailed signaling systems, beyond $\mathrm{SHH}$ signaling. This observation is in line with the fact that sporadic BCC exhibit a mostly moderate response to targeted $\mathrm{SHH}$ inhibitors. There are thus several potential drug targets already, and doubtlessly many more remain to be discovered.

\section{Part two: new topical and systemic treatment modalities for BCC}

\section{Vitamin D and NSAIDs as anticancer agents}

\section{Targeted non-invasive treatments: vitamin D}

The primary aim of BCC treatment is complete tumor eradication. However, maximal preservation of function and cosmesis at the treatment site are very important aspects that must be considered when choosing an appropriate therapy. The treatment of choice largely depends on clinical and histopathological characteristics. The risk of recurrence should be carefully evaluated in order to avoid overtreatment of low-risk BCCs or under-treatment of high-risk BCCs. ${ }^{52}$ Conventional surgical excision is the gold standard in treatment of all BCCs, with success rates varying from 90-98\% for primary (untreated) BCCs. ${ }^{53,54}$ For sBCCs, non-invasive treatment modalities such as photodynamic therapy (PDT) (photochemical), imiquimod (immune-modulating) and 5-fluorouracil (5-FU) cream (chemotherapeutic) are well studied and frequently prescribed..$^{55}$ Long term tumor-free survival rates in sBCCs are high, varying from $72.8-84.0 \%$ in PDT, $83.4-87.3 \%$ for Imiquimod and tumor-free survival rates of $80.1 \%$ were found after treatment with 5 -flourouracil. 55,56 This illustrates that cure rates following surgical excision are still much higher. At the time of the clinical trial described in chapter 4.1, there were no pertinent data to support non-invasive treatments for nBCCs. Likewise, according to the Dutch 'evidenced-based guideline treatment of the basal cell carcinoma', non-invasive treatment modalities were reserved for sBCCs only. We concluded that there was still an opportunity for (the development of) alternative or additional non-invasive treatment modalities for sBCCs as well as nBCCs.

Current research in oncology is focusing on the development of targeted cancer treatments that are specifically aimed at key signaling pathways instead of the more generally used, nonspecific cytotoxic agents. Interestingly, sometimes drugs already available for the treatment of one disease turn out to be effective against a different disease (including human cancers), which is known as drug repurposing. The active metabolite of vitamin D, 1a,25(OH)2D3 (calcitriol) is of particular interest in this regard. Preclinical research has indicated that this metabolite or other vitamin D analogues might have potential as anticancer agents because of their antiproliferative effects, activation of apoptotic pathways and inhibition of angiogenesis. ${ }^{57}$ Vitamin $D$ is known to be a steroid hormone crucial in calcium homeostasis and regulation of bone metabolism. It also has many other biological actions that are still not fully characterized. ${ }^{58}$ In the skin, calcitriol is mainly synthesized under influence of UVB exposure. 
For that reason, sun protection advice given by dermatologists in order to prevent skin aging and skin cancer is often disobeyed because patients fear becoming vitamin D deficient. Indeed, sunscreens do block the vitamin D production, however in daily practice, typically only little amounts of sunscreen are applied to the skin and vitamin D deficiency therefore is not normally an issue. Mean daily exposure during 15-30 minutes (depending on latitude and season) of the face, hands and underarms without any sun protection would generate the required amount of vitamin D in people with Fitzpatrick skin type I-III. However, dermatologists and cancer groups, including the Skin Cancer Foundation of the United States discourage such unprotected UV exposure, as all unprotected UV exposure contributes to cumulative DNA damage and thus to a further increased risk of skin cancer development. Their advice is to substitute vitamin D orally. In a time where vitamin D (deficiency) seems to be a real hype, it is important to realize that neither the influence of vitamin $\mathrm{D}$ insufficiency nor the health benefit of maintaining high serum vitamin $\mathrm{D}$ levels has been established for the general population. ${ }^{59}$ Animal and cellular studies strongly suggest a role for vitamin D in the prevention and treatment of various human cancers. So far, however, clinical studies in most cancers have not yet delivered compelling evidence to support the use of vitamin D supplements in daily practice. ${ }^{60}$

In keratinocytes, the vitamin D receptor (VDR) interferes in both $\mathrm{SH}$ - and WNT signaling and that way seems to be crucial in the tumorigenic response. ${ }^{61}$ Also, normal keratinocytes and melanocytes respond to calcitriol with a reduction in proliferation and an increase in differentiation. ${ }^{62}$ There is some evidence to suggest that high vitamin D serum levels might protect against BCC development. ${ }^{63}$ Conversely, an association with higher serum vitamin D levels and a higher risk of BCC has also been shown in two studies. ${ }^{64,65}$ This higher risk may well be explained by the fact that whereas UVB exposure does lead to higher vitamin D serum levels, it will also cause the DNA damage that results in skin cancer. ${ }^{61}$ Additionally, previous in vitro studies found that high doses of calcitriol can inhibit keratinocyte proliferation, while lower doses may stimulate proliferation. ${ }^{62,66}$ Apparently, the balance between the positive and negative effects of vitamin $D$ is a very delicate one. In our study, described in chapter 4.1, topical calcitriol applied twice daily to sBCCs and nBCCs during 8 weeks did not have any effect. No histopathological tumor clearance was found, nor was there any effect on the proliferation marker $\mathrm{Ki}-67$ and anti-apoptosis marker $\mathrm{Bcl}-2$. However, based on our results we cannot completely rule out that vitamin D analogs could treat BCC. The lack of response we observed might have been due to calcitriol being unable to reach sufficiently high concentrations in the tumors.

\section{Targeted non-invasive treatments: diclofenac}

Nonsteroidal anti-inflammatory drugs (NSAIDs) are well-known and frequently used drugs with analgesic, antipyretic and anti-inflammatory effects. There are many different types of NSAIDs. The main class effect is the inhibition of cyclooxygenases 1 (COX-1) and 2 (COX2 ), with a consequent decrease in prostaglandin synthesis. COX-1 is a housekeeping gene that is constitutively expressed and controls normal physiological functions such as maintenance of the gastric mucosa and platelet function, vascular homeostasis, as well as renal blood flow. In contrast, COX-2 is an immediate-early gene, and expression is generally be- 
low detection levels. ${ }^{67} \mathrm{COX}-2$ modulates cell proliferation, angiogenesis and suppression of apoptosis. ${ }^{68}$ In BCCs, COX-2 is commonly highly expressed under influence of UV radiation and multiple other inflammatory stimuli, whereas in normal skin COX-2 is usually undetectable. ${ }^{67,68}$ Diclofenac is the most widely prescribed non-selective COX inhibitor worldwide. ${ }^{69}$ Over the past three decades, epidemiologic, clinical, and experimental studies have established a chemopreventive effect of NSAIDs in various tissues. However NSAIDs are not used as chemopreventive agent because of incomplete efficacy and toxicities. ${ }^{70}$ The effect of the oral selective COX-2 inhibitor Celecoxib on the development of BCCs was studied in a double-blinded, randomized controlled clinical trial in 60 patients with BCNS. After Celecoxib (200 mg, twice daily, 24 months) a trend towards reduction in BCC burden in the treatment group in all subjects was sugested. ${ }^{71} \mathrm{~A}$ recent meta-analysis did not find a statistically significant chemopreventive effect of NSAIDs on NMSC, not even after stratification for the different NSAIDs and for BCCs and SCCs. ${ }^{72}$ Thus, there is no convincing evidence to date that regular use of systemic NSAIDs could serve as chemopreventive agent in the development of BCCs. However, as the local bioavailability is expected to be higher after local application, and considering the fact that NSAIDs can target the WNT signaling pathway that is activated in $\mathrm{BCCs}^{73}$, we assumed that topically applied diclofenac could be effective in the treatment of (low-risk) BCCs. Moreover, diclofenac both directly and indirectly induces apoptosis. ${ }^{74,75}$ In chapter 4.1 we showed that application of diclofenac $3 \%$ gel with $2.5 \%$ hyaluronic acid for 8 weeks twice daily (under occlusion) to sBCC resulted in a complete histologic regression in $64.3 \%$ of all tumors. These results further support inflammation as a possible driver of BCC growth (see also part one). The histological clearance found was not as high as can be obtained with currently available non-invasive treatment modalities, however it should be noted that we made use of a diclofenac compound (Solaraze ${ }^{\circ}$ ) that is already FDA approved for the treatment of actinic keratosis (AK). ${ }^{76}$ Previous dose-response studies for its specific use in BCC were not performed. Optimized dosage regimens might further improve efficacy. Apart from more targeted mode of action, further advantages of topical diclofenac are that it is cost-efficient, easy in use and that it has limited side effects. This makes topical diclofenac a promising drug for the treatment of (low-risk) BCCs.

\section{Topical diclofenac as chemopreventative agent}

Because of the promising results of topical diclofenac as treatment of sBCC and the ever larger number of patients affected, it is of interest to determine whether topical diclofenac could serve as a chemopreventative agent. About one third of the patients diagnosed with their first primary BCC will develop at least one subsequent BCC. This risk is highest within the first 6 months after diagnosis, but remains substantially high even after a follow-up period of 5 years. In particular patients with red hair, a higher socioeconomic status, and/or those with a BCC on their upper extremities have a higher risk of developing multiple BCCs. ${ }^{77}$

Topically applied $3 \%$ diclofenac gel with $2.5 \%$ hyaluronic acid (the same formula used in the clinical trial in chapter 4.1) has already been used in the treatment of AK and resulted in complete clinical clearance of $41-60 \%$ of AKs after 3 months of treatment. ${ }^{76}$ Topical diclofenac in 
twice-daily doses is approved by the FDA for the treatment of AK over a period of 60-90 days and it is prescribed for this purpose in many European countries, although its use is not recommended by the 'Dutch guideline actinic keratosis'. Given the inhibitory effect of diclofenac on cell proliferation and the promising response rates both in $\mathrm{AK}$ and $\mathrm{BCC}$, topical diclofenac could presumably help to reduce the risk of new or subsequent BCC development. The side effects are usually mild to moderate (e.g. erythema, pruritus and erosion) and resolve without sequelae (chapter 4.1). Therefore, it might be of interest to explore whether diclofenac could be used as a preventive additive to daily skin care creams. With a lower concentration or a reduced frequency of application, known side effects could be reduced whilst retaining efficacy.

\section{Targeted non-invasive treatments and future perspectives of BCC management}

In contrast to most cancers, skin tumors are readily accessible for clinical examination and tissue samples for (immuno)histochemical $(\mathrm{IHC})$ evaluation can easily be obtained. It might be argued that accurate clinical follow-up instead histological examination could be considered when assessing new topical treatments in clinical trials. However, immunohistochemical stains on pre- and post-treatment tissue samples can provide insight in the mode of action or efficacy of new treatment modalities. In quantifying the expression levels of specific immunohistochemical markers, percentages of positive tumor cells need to be determined. Manual pathologist-based quantification $(\mathrm{MQ})$ is still the most frequently used technique for $\mathrm{IHC}$ evaluation ${ }^{78}$ and is considered as the gold standard. However, MQ is time-consuming and labor-intensive. ${ }^{79}$ It is also a subjective approach that may have high intra- and inter-observer variability. ${ }^{80-82}$ Larger studies with more immunohistochemical stains to be investigated inevitably generate a large number of slides to be assessed, rendering MQ impractical.

Automated, digital image analysis might offer a solution and has been used since the 1980s. Several different methods are available. ${ }^{83}$ We used a so-called operator-dependent semi-automated quantification (SAQ) computerized thresholding technique (Leica Qwin version 3.5.1, Leica Microsystems, Cambridge, UK), which is an extensively evaluated method, especially for mammographic density. ${ }^{84,85} \mathrm{SAQ}$ is time saving, provides easily accessible data and generates good reproducible results, even by generally inexperienced young researchers (chapter 4.2). However, we found large discrepancies between $M Q$ and $S A Q$, that point to the need for studies in which consensus evaluation by more than one pathologist is compared with SAQ measurements. Evidence from a well-designed study that SAQ is a reproducible and accurate method for assessing immunohistochemical stains would be very relevant for research. Studies assessing changes in expression values in pre- versus post-treatment tissue samples are especially suitable for $S A Q$, as the change in expression is less susceptible for random errors.

In chapter 4.1, two pathologists manually assessed all pre- and post-treatment tissue samples. In contrast to the more promising results in sBCCs, in nBCCs we did not detect significant changes in proliferation, apoptosis or total tumor clearance after treatment with diclofenac, calcitriol or a combination of both. The difference in response between nBCCs and 
sBCCs is in agreement with reports from other studies investigating non-invasive therapies. sBCCs spread from the epidermis into the papillary dermis, which makes the tumors more accessible to topical treatment. In contrast, nBCCs show deep, compact, and nodular growth ${ }^{86}$ presumably leading to insufficient penetration of topically applied drugs. Only recently, high 3-year success rates (81.8\%) were found for nBCCs treated with imiquimod for 12 weeks, 7 days a week. ${ }^{54}$ This is an intensified treatment schedule compared to the presently licensed schedule of 5 days a week for 6 weeks. Imiquimod has several antitumor activities. The major biological effect of imiquimod is the activation of nuclear factor-kappa B (NF-kB) through toll-like receptors 7 (TLR7) and 8 (TLR8). Induction of NF-KB results in the production and secretion of pro-inflammatory cytokines and chemokines, stimulating a strong Th1-mediated antitumoral cellular immune response. ${ }^{87}$ Additionally, imiquimod directly stimulates antitumoral activation of dendritic cells (DCs). DCs are important (tumor) antigen presenting cells in the skin and they are thought to be crucial in generating an aggressive immune response against cutaneous cancers. ${ }^{51}$ Interestingly, a recent study in a Patched 1 deficient murine BCC cell line suggests that imiquimod may actually serve as a targeted therapy by directly inhibiting $\mathrm{SHH}$ signaling downstream of SMO. Imiquimod can cause GLI phosphorylation and subsequent reduction in GLI target genes. ${ }^{88}$ The fact that imiquimod, apart from its immunomodulating effect, also seems to directly inhibit SHH signaling, could explain why it is the most effective topical treatment currently available. ${ }^{55}$ Indeed (pre-) clinical evidence has suggested that simultaneously targeting $\mathrm{SHH}$ and other signaling pathways may have a synergistic effect. ${ }^{89}$ In chapter 4.1 we hypothesized that combining diclofenac gel and calcitriol ointment would enhance their individual efficacy. In our clinical trial, the combination did not show the expected synergistic effect. The observed clinical effect of the "combination therapy" arm was probably attributable to diclofenac, as there was no effect at all in the calcitriol arm. Since calcitriol ointment was applied five minutes after application of diclofenac gel, dilution or suboptimal absorption could explain the observed reduced efficacy compared to diclofenac monotherapy in sBCCs. Of note, in the combination therapy group, there were four non-responding tumors that appeared to have a nodular BCC component although they were initially diagnosed as sBCC on the biopsy. Since we also found that nodular BCCs did not respond to topical therapy, treatment failure in these patients was probably related to the histological subtype. Due to the sample size of 16 tumors per arm, those 4 non-responders with a different histological subtype had a large impact on the overall treatment outcome. Nevertheless, the promising results of topical diclofenac in sBCCs suggest new possibilities for the management of BCCs. Further optimization with dose-response trials and studies combining topical diclofenac with available (targeted) non-invasive therapies are needed to test whether topicals can obtain cure rates comparable to those of the gold standard, surgery. Based on our present understanding, treatment with diclofenac gel and imiquimod cream is a promising combination that we are currently investigating.

Non-invasive treatments in general can be expected to have several advantages, making them worth pursuing. They will generally give a better cosmetic outcome, with an increased quality of life for patients as a result. ${ }^{54}$ Patients will be able to treat themselves at home, which makes them less dependent on hospital care. A second advantage of this home- 
based treatment is that the workload for dermatologists will decrease, resulting in lower overall healthcare costs. Since the skin cancer epidemic is yet to peak, the workload for dermatologists is expected to increase. General practitioners might be able to help in this increase in demand. However, according to a self-administered questionnaire filled in by 268 general practitioners, over $50 \%$ of the general practitioners felt the need to know more about skin cancer care..$^{90}$ Intensified training programs on recognition and management of skin cancer are therefore essential to facilitate treatment by non-specialists. General practitioners are already used to take biopsies of suspicious lesions, but they generally do not perform therapeutic surgical excisions of BCCs (yet). Also, in general, they are not familiar with prescribing non-invasive treatments. Good collaborations between the general practitioners and dermatologists are necessary to improve care of the patient with (pre-) skin cancer, and is essential in order to reduce health care costs. The department of dermatology (MUMC) already collaborates with general practitioners in the so called ' $1.5^{\text {th }}$ line of care' or 'stadspoliklinieken', where dermatologists visit the general practitioner one day a week in order to facilitate dermato(onco-)logical care by reducing the number of unnecessary referrals to the hospital. Another way to make current dermatological practice more efficient is the employment of specialized nurse practitioners and physician assistants, who could participate in the management of skin cancer. Of course, oncological care of the skin should then be thoroughly implemented in their educational training. With the appropriate knowledge, these paramedics could not only educate patients about skin cancer prevention, but they would also be able to recognize (pre-) skin malignancies and potentially even treat AK and low-risk BCCs. ${ }^{91}$ Of note, high risk BCC, SCC and malignant melanoma should always be treated by a dermatologist, as they are associated with higher morbidity and even mortality. However, paramedics could serve as a first point of call for BCC patients and help manage further care. It should be stressed that paramedics should always collaborate with a dermatologist.

\section{Targeted SMO-inhibitors in BCC}

\section{Vismodegib and (acquired) resistance}

$\mathrm{BCC}$ is characterized by abberant activation of the $\mathrm{SHH}$ pathway, mostly due to inactivating mutations in the tumor suppressor gene PTCH1 or activating mutations in the SMO oncogene. ${ }^{92} \mathrm{SHH}$-inhibitors specifically target $\mathrm{SMO}$, but GLI or other downstream pathway components may be targeted in the near future. ${ }^{93}$ Vismodegib (Erivedge ${ }^{\mathrm{TM}}$, also known as GDC0449) is an orally active synthetic SMO inhibitor and was approved by the FDA in 2012 for the treatment of laBCC and mBCC. ${ }^{94}$ In July 2015, a second SMO inhibitor, sonidegib (OdomzO ${ }^{\mathrm{TM}}$, also known as LDE255), was approved by the FDA for laBCC not amenable to curative surgery or radiation therapy. ${ }^{95}$ As the mode of action of these Hedgehog Pathway Inhibitors (HPIs) is identical, similar efficacy rates and adverse events are seen (class effect). ${ }^{95,96}$ Recently, preplanned interim results of the largest international, open label trial to the safety and efficacy of vismodegib in laBCC and mBCC (STEVIE study) were published. ${ }^{97}$ After at least 12 months of treatment, complete and partial response was seen in 32\% of laBCC and $33 \%$ of $\mathrm{mBCC}$ patients. Twenty-seven percent of all patients treated had stable disease and progressive 
disease was seen in $3 \%$ of the patients. The mean duration of treatment was 36.3 weeks for laBCCs and 52.0 weeks for mBCCs. Treatment was discontinued in no less than $80 \%$ of all patients treated in this study. Almost all patients reported one or more adverse events (99\%), of which the most important events where muscle spasms, alopecia and dysguesia (loss of taste). These adverse events were the most important reason for treatment discontinuation. The authors concluded that the safety profile described in the STEVIE study was consistent with previous analyses of vismodegib in patients with laBCC and $\mathrm{mBCC} .{ }^{97}$ The department of dermatology of the MUMC participated in this STEVIE trial and included 6 patients.

Initial response to vismodegib followed by secondary tumor progression has been observed and is suggested to occur in $20 \%$ of the patients within the first year of treatment. ${ }^{98-102}$ The mechanisms underlying resistance to vismodegib in BCCs may clinically be divided into primary resistance (no response to treatment) or secondary (acquired) resistance (progression after an initial response). ${ }^{103}$ We purposely speak of a clinical classification, because it is still unclear whether the non-responding tumor cells are already present in the primary tumor and grow out to visible tumor due to selection, or whether HPIs may even induce mutations that lead to resistance. Acquired resistance to vismodegib has also been described in medulloblastoma, caused by a heterozygous SMO mutation that appeared in the metastatic medulloblastoma during treatment. ${ }^{104}$ In this thesis, two patients with laBCCs who had developed respectively primary and secondary resistance to vismodegib are outlined. Chapter $\mathbf{5 . 1}$ describes two heterozygous mutations ((p.Trp281Leu and p.Val321Met) in newly developed tumor tissue after 20 weeks of treatment in a laBCC patient who initially had a good clinical response to vismodegib. Neither SMO mutation was found in the laBCC biopsy specimen obtained before initiation of vismodegib therapy, or in the biopsy specimens of responding sclerotic skin during therapy. Analysis of a buccal swab revealed no mutations at all. It is of particular interest that the same novel somatic $\mathrm{PTCH} 1$ mutation was detected in both the primary and the resistant BCC, and was absent in clinically and histologically responding tissue. This observation implies that cells from the original tumor had survived. Thus, even though the resistant tumors arose from the same, clonal primary tumor, a clonal origin of the resistant BCC nests is precluded, a finding that supports tumor heterogeneity. Interestingly, the SMO codon 321 that we found was previously described as a critical binding site for vismodegib binding ${ }^{102}$, but codon 281 has never been related to vismodegib resistance. It appears that resistance to vismodegib in BCC can be caused by several different acquired SMO mutations that interfere with drug binding, a finding that is also described by Sharpe et.al. ${ }^{92}$ Also, in a few resistant cases, recurrent copy number variants in the downstream effectors SUFU and Gli2 were detected. There is a complex interplay between mutual and heterogeneous resistance mechanisms to SMO inhibition in BCCs, even within the same tumor. ${ }^{92}$ The fact that sporadic BCC is a very heterogeneous tumor is confirmed by whole-exome sequencing of the genetic landscape of BCC, showing that BCCs harbor the most mutations of all known human cancers. ${ }^{28}$ From this point of view, it could be suggested that the use of oral SMO inhibitors like vismodegib should be reserved for BCNS patients only, given the fact that these BCC are presumed to be obligatory and solely $\mathrm{SHH}$ driven, in contrast with sporadic BCC that appears to be more multifactorial (see part one of this discussion). 
A better understanding of the tumorigenesis of sporadic BCC and the mechanisms underlying vismodegib resistance is important for the development of alternative treatment strategies. Sequential mutation analysis on pre-treatment and recurrent tumor tissue may help to anticipate on the type of resistance (either primary or secondary) in order to actively alter therapy. Then, treatment schedules and combination therapies can be customized to both patient and tumor characteristics, in an example of so-called personalized medicine. Overall, possible therapeutic approaches are sequential or rotational therapy with non-treatment periods ('drug holidays') in order to obtain a better tolerability for side effects, combination therapies, or alternating different therapies. Combining different systemic therapies in laBCC or mBCC may have the advantage of synergistic or additional therapeutic effects, lower dosage application and, possibly, the prevention of (acquired) resistance. ${ }^{105}$ In addition, systemic itraconazole and nicotinamide might be promising agents in BCC treatment. ${ }^{106,107}$

\section{Vismodegib, SCC and future analyses}

Chapter $\mathbf{5 . 2}$ discusses a patient with a 15-year history of a recurrent infiltrative BCC of the nose and right cheek, who was included in the STEVIE study and treated with vismodegib. No clinical improvement was observed and we even feared for progression into SCC as impressive hyperkeratosis developed after 7 weeks of treatment. Formation of SCC within the initial BCC has been reported in several case studies describing patients treated with vismodegib, as has development of SCC and keratoacanthoma on other body sites. ${ }^{108-113}$ In our patient, histologic examination of tissue specimens acquired during surgery performed 5 months after initiation of vismodegib treatment, revealed residual BCC cells with positive staining for staining Ber-Ep4 (BCC marker). No signs of basosquamous carcinoma or SCC were found. Also, there were epidermal cysts that stained negatively for Ber-Ep4, excluding the possibility that these were BCC remnants that had survived of the original tumor. A possible explanation for the emergence of the benign squamous neoplasms could be the effect of vismodegib on keratinocyte differentiation. Vismodegib can mimic the loss of Indian hedgehog $(\mathrm{IH})$, a member of the $\mathrm{HH}$ ligand family the loss of which was shown to promote the progression of benign papillomas to SCC. ${ }^{114}$ In the case of SCCs emerging upon SMO inhibition, it has been hypothesized that a phenotypic switch from BCC to SCC, caused by new mutations (NOTCH1/2 and KMTC2), could occur as a mechanism of tumor escape. ${ }^{115}$ Secondly, SMO inhibition might promote carcinogenesis by selecting tumor cells driven by other molecular pathways, like RAS/MAPK signaling. Activation of RAS/MAPK signaling can override the oncogenic addiction of the tumor to $\mathrm{SHH}$ signaling and thereby enabling proliferation of resistant tumors with enhanced metastatic behavior. ${ }^{116}$ However, further functional analysis is needed if the complex effects of vismodegib on keratinocyte differentiation and proliferation. Unfortunately, we were not able to perform any mutational analyses in our patient, mostly because no pre-treatment fresh frozen tissue samples had been obtained.

In daily dermatological and pathological practice, tissue samples acquired for diagnostic considerations are generally formalin-fixed paraffin-embedded (FFPE). DNA extracted from FFPE is fragmented and can also contain sequence artifacts arising from DNA dam- 
age that can be difficult to distinguish from true mutations, especially in highly heterogeneous tumors such as BCC. These sequence variants are more often seen in FFPE than in fresh frozen tissue. ${ }^{117}$ One of the potential techniques to overcome this problem is the use of molecular inversion probes (MIPS). MIPs provide a practical, highly sensitive method for the detection of low-frequency mutations and subclonal variations in FFPE, requiring low amount of sample input, while per-sample costs are very low. ${ }^{118}$ This technique makes it possible to retrospectively perform pre- and post-treatment analysis. Such approaches will help to further unravel mechanisms of resistance, not only to vismodegib in BCC, but also to other small molecule inhibitors used to treat human malignancies such as melanoma.

\section{Vismodegib and its place in daily dermatological practice}

Systemic targeted treatments such as vismodegib are associated with potential toxicities and the number of patients reporting adverse events is considerably high, making its use limited to laBCCs and mBCCs. However an international multicenter clinical trial to the efficacy and safety of two different vismodegib regimens in patients with multiple BCC (MIKIE) (NCT01815840, clinicaltrials.gov) is currently ongoing. This study already anticipates on the fact that this group of patients will require a long-term treatment by introducing rotational therapy in which 'drug-holidays' of 8 weeks are implemented in an attempt to minimize adverse events. It can be speculated that also the occurrence of resistance may be diminished following rotational therapy. The interim results of the STEVIE study showed a median time to first onset of the most common adverse events of 2.83 months for muscle spasms, 5.55 months for alopecia, and 6.51 months for dysgeusia. ${ }^{96}$ The side effects are cumulative and tend to get worse with increasing duration of the treatment. Temporary treatment stops e.g. every 3 months will lower or even prevent the burden of adverse events and is specially desirable in patients with multiple BCCs and BCNS. The possibility to use vismodegib in alternating treatment schedules in order to support a longer maintenance on treatment would provide an extra indication for FDA approval for this indication. However, experts in the field already implement drug-holidays, especially in BCNS patients, in which the treatment regimen is personalized to the patient and mainly patient driven rather than doctor driven. Interesting is a phase II, single-armed, multicenter trial (the $\mathrm{NICCI}$ trial), assessing the utility of vismodegib as neo-adjuvant treatment prior to surgery in laBCC and rBCC. ${ }^{119}$ Although currently still off-label, limited case reports already describe results of neo-adjuvant treatment of laBCCs with vismodegib. ${ }^{98,120,121} \mathrm{Neo}$-adjuvant therapy with vismodegib was effective in reducing the surgical defect, leading to less scarring and morbidity. However, long term follow-up is lacking and BCC remnants were found during Mohs surgery in the majority of the cases after three to six months of treatment with vismodegib. ${ }^{98,120,121}$ One should be aware of the fact that reduction of BCC nests following vismodegib not necessarily occurs from the periphery to the center of the tumor. Thus when performing Mohs surgery of only the center of the clinically remaining tumor, (subclinical) scattered tumor nests in het former tumor area are probably missed. This inevitably results in inadequate treatment and a high risk of recurrence. If therapy is optimized possibly by combination therapy, and the percentage of resistance for therapy is decreased, we do agree that planning patients for surgical excision 
may reduce the chance of recurrence and could offer interesting further treatment options. Currently, the use of vismodegib as systemic treatment is limited to laBCCs and mBCCs because of potential toxicities which do not outweigh the disadvantages of available treatments of regular low-risk BCCs. In these low risk tumors, topical SMO inhibitors would have a broader applicability, however the results of their effectiveness in humans are rather disappointing. Topical CUR61414 had little or no effect on proliferation and apoptosis of BCCs, assessed by Ki-67 and cleaved caspase- 3 immunohistochemical stains respectively, probably due to low penetration or rapid clearance of the active component. ${ }^{122}$ LDE225 $0.75 \%$ cream is a different topical SMO inhibitor and although clinical regression is seen in almost all BCCs of eight BCNS patients after 4 weeks of treatment, histological examination of post-treatment biopsy samples of all tumors revealed that tumor nests were still present. A major advantage of topical application is the excellent safety profile with no local or systemic side effects. ${ }^{123}$ Topical SMO inhibitors could emerge as an attractive non-invasive alternative for low-risk BCCs. However, future studies are needed to improve their efficacy and provide confident evidence for their use in daily practice.

The development and availability of small-molecule (targeted) inhibitors as vismodegib offer a new area in the management of BCC. As the dermatologist is the specialists with the most experience in treatment of different forms of skin cancer and the only specialist that has accessibility to all available treatment options, the dermatologist should always be involved in setting the indication for such treatments. In any case a multidisciplinary team in which the dermatologist, medical oncologist, plastic surgeon, radiotherapist and ENT-specialist closely cooperate should be involved. As laBCC/mBCC is generally a disease of the elderly, comorbidities and polypharmacy are not uncommon. The management of these patients requires a more holistic approach. Some have argued that the prescription and treatment with vismodegib should be restricted to medical oncologists. However, most dermatologists are experienced in prescribing systemic drugs and even cytostatic drugs and they are capable of dealing with possible side effects and adverse events. Treatment should be supervised by the physician who is experienced in the patient's ill-health and it is likely that the physician who decides to start vismodegib is the main therapist. In mBCCs, this could be the medical oncologist, but in laBCCs treatment by the dermatologists seems more logical. A good collaboration between all members of the multidisciplinary team is a key point. As the number of patients is limited, it is important to gain and share experiences with all team members and discuss cases regularly. 


\section{References}

1. Esteller M. Epigenetics in cancer. The New England journal of medicine 2008;358:1148-59.

2. Rodriguez-Paredes M, Esteller M. Cancer epigenetics reaches mainstream oncology. Nature medicine 2011;17:330-9.

3. Portela A, Esteller M. Epigenetic modifications and human disease. Nature Biotechnology 2010;28:1057-68.

4. Goldberg M, Rummelt C, Laerm A, Helmbold P, Holbach LM, Ballhausen WG. Epigenetic silencing contributes to frequent loss of the fragile histidine triad tumour suppressor in basal cell carcinomas. The British journal of dermatology 2006;155:1154-8.

5. Heitzer E, Bambach I, Dandachi N, Horn M, Wolf P. PTCH promoter methylation at low level in sporadic basal cell carcinoma analysed by three different approaches. Experimental dermatology 2010;19:926-8.

6. Sathyanarayana UG, Moore AY, Li L, et al. Sun exposure related methylation in malignant and non-malignant skin lesions. Cancer letters 2007;245:112-20.

7. van Doorn R, Gruis NA, Willemze R, van der Velden PA, Tensen CP. Aberrant DNA methylation in cutaneous malignancies. Seminars in oncology 2005;32:479-87.

8. Wu W, Zhang J, Yang H, Shao Y, Yu B. Examination of AKAP12 promoter methylation in skin cancer using methylation-sensitive high-resolution melting analysis. Clinical and experimental dermatology 2011;36:3815 .

9. Stamatelli A, Vlachou C, Aroni K, Papassideri I, Patsouris E, Saetta AA. Epigenetic alterations in sporadic basal cell carcinomas. Archives of dermatological research 2014;306:561-9.

10. Katiyar SK, Singh T, Prasad R, Sun Q, Vaid M. Epigenetic alterations in ultraviolet radiation-induced skin carcinogenesis: interaction of bioactive dietary components on epigenetic targets. Photochemistry and photobiology 2012;88:1066-74.

11. Ikehata H, Ono T. The mechanisms of UV mutagenesis. Journal of Radiation Research 2011;52:115-25.

12. Flohil SC, Seubring I, van Rossum MM, Coebergh JW, de Vries E, Nijsten T. Trends in Basal Cell Carcinoma Incidence Rates: A 37-Year Dutch Observational Study. The Journal of investigative dermatology 2012.

13. Schuebel KE, Chen W, Cope L, et al. Comparing the DNA hypermethylome with gene mutations in human colorectal cancer. PLoS genetics 2007;3:1709-23.

14. Helmbold P, Richter AM, Walesch S, et al. RASSF10 promoter hypermethylation is frequent in malignant melanoma of the skin but uncommon in nevus cell nevi. The Journal of investigative dermatology 2012;132:687-94.

15. Helmbold P, Lahtz C, Enk A, et al. Frequent occurrence of RASSF1A promoter hypermethylation and Merkel cell polyomavirus in Merkel cell carcinoma. Molecular carcinogenesis 2009;48:903-9.

16. Esteller M. Aberrant DNA methylation as a cancer-inducing mechanism. Annu Rev Pharmacol Toxicol 2005;45:629-56.

17. Derks S, Lentjes MH, Hellebrekers DM, de Bruine AP, Herman JG, van Engeland M. Methylation-specific PCR unraveled. Cell Oncol 2004;26:291-9.

18. Harris RA, Wang T, Coarfa C, et al. Comparison of sequencing-based methods to profile DNA methylation and identification of monoallelic epigenetic modifications. Nature Biotechnology 2010;28:1097-105.

19. Mensaert K, Denil S, Trooskens G, Van Criekinge W, Thas O, De Meyer T. Next-generation technologies and data analytical approaches for epigenomics. Environ Mol Mutagen 2014;55:155-70.

20. Sandoval J, Heyn H, Moran S, et al. Validation of a DNA methylation microarray for 450,000 CpG sites in the human genome. Epigenetics 2011;6:692-702.

21. De Meyer T, Mampaey E, Vlemmix M, et al. Quality evaluation of methyl binding domain based kits for enrichment DNA-methylation sequencing. PloS one 2013;8:e59068.

22. Brinkman AB, Simmer F, Ma K, Kaan A, Zhu J, Stunnenberg HG. Whole-genome DNA methylation profiling using MethylCap-seq. Methods 2010;52:232-6. 
23. van Vlodrop IJ, Niessen HE, Derks S, et al. Analysis of promoter CpG island hypermethylation in cancer: location, location, location! Clinical cancer research : an official journal of the American Association for Cancer Research 2011;17:4225-31.

24. Rausch MP, Hastings KT. GILT modulates CD4+ T-cell tolerance to the melanocyte differentiation antigen tyrosinase-related protein 1. The Journal of investigative dermatology 2012;132:154-62.

25. Ciurea ME, Cernea D, Georgescu CC, et al. Expression of CXCR4, MMP-13 and beta-catenin in different histological subtypes of facial basal cell carcinoma. Romanian journal of morphology and embryology = Revue roumaine de morphologie et embryologie 2013;54:939-51.

26. Tarca AL, Draghici S, Khatri P, et al. A novel signaling pathway impact analysis. Bioinformatics 2009;25:7582.

27. Muehleisen B, Jiang SB, Gladsjo JA, Gerber M, Hata T, Gallo RL. Distinct innate immune gene expression profiles in non-melanoma skin cancer of immunocompetent and immunosuppressed patients. PloS one 2012; 7:e40754.

28. Jayaraman SS, Rayhan DJ, Hazany S, Kolodney MS. Mutational landscape of basal cell carcinomas by whole-exome sequencing. The Journal of investigative dermatology 2014;134:213-20.

29. Kasiske BL, Snyder JJ, Gilbertson DT, Wang C. Cancer after kidney transplantation in the United States. American journal of transplantation : official journal of the American Society of Transplantation and the American Society of Transplant Surgeons 2004;4:905-13.

30. Walter A, Barysch MJ, Behnke S, et al. Cancer-testis antigens and immunosurveillance in human cutaneous squamous cell and basal cell carcinomas. Clinical cancer research : an official journal of the American Association for Cancer Research 2010;16:3562-70.

31. Zavos G, Karidis NP, Tsourouflis G, et al. Nonmelanoma skin cancer after renal transplantation: a single-center experience in 1736 transplantations. International journal of dermatology 2011;50:1496-500.

32. Wheless L, Jacks S, Mooneyham Potter KA, Leach BC, Cook J. Skin cancer in organ transplant recipients: more than the immune system. Journal of the American Academy of Dermatology 2014;71:359-65.

33. Carlson JA, Combates NJ, Stenn KS, Prouty SM. Anaplastic neoplasms arising from basal cellcarcinoma xenotransplants into SCID-beige mice. Journal of cutaneous pathology 2002;29:268-78.

34. Urosevic M, Dummer R. Immunotherapy for nonmelanoma skin cancer: Does it have a future? Cancer 2002;94:477-85.

35. Fujimura T, Kakizaki A, Kambayashi Y, Aiba S. Basal cell carcinoma with spontaneous regression: a case report and immunohistochemical study. Case Reports in Dermatology 2012;4:125-32.

36. Kaporis HG, Guttman-Yassky E, Lowes MA, et al. Human basal cell carcinoma is associated with Foxp3+ T cells in a Th2 dominant microenvironment. The Journal of investigative dermatology 2007;127:2391-8.

37. Heyn H, Esteller M. DNA methylation profiling in the clinic: applications and challenges. Nature reviews Genetics 2012;13:679-92.

38. Orr BA, Haffner MC, Nelson WG, Yegnasubramanian S, Eberhart CG. Decreased 5-hydroxymethylcytosine is associated with neural progenitor phenotype in normal brain and shorter survival in malignant glioma. PloS one 2012; 7:e41036.

39. Shukla A, Sehgal M, Singh TR. Hydroxymethylation and its potential implication in DNA repair system: A review and future perspectives. Gene 2015;564:109-18.

40. Romani M, Pistillo MP, Banelli B. Environmental Epigenetics: Crossroad between Public Health, Lifestyle, and Cancer Prevention. BioMed research international 2015;2015:587983.

41. Marks PA, Breslow R. Dimethyl sulfoxide to vorinostat: development of this histone deacetylase inhibitor as an anticancer drug. Nat Biotechnol 2007;25:84-90.

42. Mottamal M, Zheng S, Huang TL, Wang G. Histone deacetylase inhibitors in clinical studies as templates for new anticancer agents. Molecules 2015;20:3898-941.

43. Canettieri G, Di Marcotullio L, Greco A, et al. Histone deacetylase and Cullin3-REN(KCTD11) ubiquitin ligase interplay regulates Hedgehog signalling through Gli acetylation. Nature cell biology 2010;12:132-42. 
44. Zhao J, Quan H, Xie C, Lou L. NL-103, a novel dual-targeted inhibitor of histone deacetylases and hedgehog pathway, effectively overcomes vismodegib resistance conferred by Smo mutations. Pharmacology Researcg and Perspectives 2014;2:e00043.

45. Mansoori B, Sandoghchian Shotorbani S, Baradaran B. RNA interference and its role in cancer therapy. Advanced Pharmaceutical Bulletin 2014;4:313-21.

46. Peterson SC, Eberl M, Vagnozzi AN, et al. Basal cell carcinoma preferentially arises from stem cells within hair follicle and mechanosensory niches. Cell Stem Cell 2015;16:400-12.

47. Evans SM, Schrlau AE, Chalian AA, Zhang P, Koch CJ. Oxygen levels in normal and previously irradiated human skin as assessed by EF5 binding. The Journal of investigative dermatology 2006;126:2596-606.

48. van Steensel MA, van Geel M, Badeloe S, Poblete-Gutierrez P, Frank J. Molecular pathways involved in hair follicle tumor formation: all about mammalian target of rapamycin? Experimental dermatology 2009;18:18591.

49. Dodd KM, Yang J, Shen MH, Sampson JR, Tee AR. mTORC1 drives HIF-1alpha and VEGF-A signalling via multiple mechanisms involving 4E-BP1, S6K1 and STAT3. Oncogene 2015;23;34(17):2239-50.

50. Wataya-Kaneda M. Mammalian target of rapamycin and tuberous sclerosis complex. Journal of dermatological science 2015;79:93-100.

51. Cargnello M, Tcherkezian J, Roux PP. The expanding role of mTOR in cancer cell growth and proliferation. Mutagenesis 2015;30:169-76.

52. Marzuka AG, Book SE. Basal Cell Carcinoma: Pathogenesis, Epidemiology, Clinical Features, Diagnosis, Histopathology, and Management. Yale Journal of Biology and Medicine 2015;88:167-79.

53. Evidenced-based guideline Treatment of the basal cell carcinoma. at http://www.nvpc.nl/uploads/stand/ 150414DOC-MB-Definitieve_richtlijn_Basaalcelcarcinoom_2014_goedgekeurd_ALV_14_april_2015154.pdf.)

54. Bath-Hextall F, Ozolins M, Armstrong SJ, et al. Surgical excision versus imiquimod $5 \%$ cream for nodular and superficial basal-cell carcinoma (SINS): a multicentre, non-inferiority, randomised controlled trial. The lancet oncology 2014;15:96-105.

55. Arits AH, Mosterd K, Essers BA, et al. Photodynamic therapy versus topical imiquimod versus topical fluorouracil for treatment of superficial basal-cell carcinoma: a single blind, non-inferiority, randomised controlled trial. The lancet oncology 2013;14:647-54.

56. Roozeboom MH, Arits AH, Nelemans PJ, Kelleners-Smeets NW. Overall treatment success after treatment of primary superficial basal cell carcinoma: a systematic review and meta-analysis of randomized and nonrandomized trials. The British journal of dermatology 2012;167:733-56.

57. Deeb KK, Trump DL, Johnson CS. Vitamin D signalling pathways in cancer: potential for anticancer therapeutics. Nature reviews Cancer 2007;7:684-700.

58. Mabey T, Honsawek S. Role of Vitamin D in Osteoarthritis: Molecular, Cellular, and Clinical Perspectives. International Journal of Endocrinol 2015;2015:383918.

59. Reddy KK, Gilchrest BA. What is all this commotion about vitamin D? The Journal of investigative dermatology 2010;130:321-6.

60. Bikle DD. Vitamin D and cancer: the promise not yet fulfilled. Endocrine 2014;46:29-38.

61. Bikle DD. The vitamin D receptor: a tumor suppressor in skin. Discovery Medicine 2011;11:7-17.

62. Bikle DD, Oda Y, Xie Z. Vitamin D and skin cancer: a problem in gene regulation. The Journal of steroid biochemistry and molecular biology 2005;97:83-91.

63. Tang JY, Parimi N, Wu A, et al. Inverse association between serum 25(OH) vitamin D levels and non-melanoma skin cancer in elderly men. Cancer Causes Control 2010;21:387-91.

64. Asgari MM, Tang J, Warton ME, et al. Association of prediagnostic serum vitamin D levels with the development of basal cell carcinoma. The Journal of investigative dermatology 2010;130:1438-43.

65. Eide MJ, Johnson DA, Jacobsen GR, et al. Vitamin D and nonmelanoma skin cancer in a health maintenance organization cohort. Archives of dermatology 2011;147:1379-84. 
66. Bollag WB, Ducote J, Harmon CS. Biphasic effect of 1,25-dihydroxyvitamin D3 on primary mouse epidermal keratinocyte proliferation. Journal of cellular physiology 1995;163:248-56.

67. Muller-Decker K. Cyclooxygenase-dependent signaling is causally linked to non-melanoma skin carcinogenesis: pharmacological, genetic, and clinical evidence. Cancer metastasis reviews 2011;30:343-61.

68. Reinau D, Surber C, Jick SS, Meier CR. Nonsteroidal anti-inflammatory drugs and the risk of nonmelanoma skin cancer. International journal of cancer 2015;137:144-53.

69. McGettigan P, Henry D. Use of non-steroidal anti-inflammatory drugs that elevate cardiovascular risk: an examination of sales and essential medicines lists in low-, middle-, and high-income countries. PLoS Medicine 2013;10:e1001388.

70. Gurpinar E, Grizzle WE, Piazza GA. NSAIDs inhibit tumorigenesis, but how? Clinical cancer research : an official journal of the American Association for Cancer Research 2014;20:1104-13.

71. Tang JY, Aszterbaum M, Athar M, et al. Basal cell carcinoma chemoprevention with nonsteroidal anti-inflammatory drugs in genetically predisposed PTCH1+/- humans and mice. Cancer prevention research (Philadelphia), Pa;3:25-34.

72. Zhang B, Liang $X, Y e ~ L$, Wang $Y$. No chemopreventive effect of nonsteroidal anti-inflammatory drugs on nonmelanoma skin cancer: evidence from meta-analysis. PloS one 2014;9:e96887.

73. Roos J, Grosch S, Werz O, et al. Regulation of tumorigenic Wnt signaling by cyclooxygenase-2, 5-lipoxygenase and their pharmacological inhibitors: A basis for novel drugs targeting cancer cells? Pharmacology and Therapeutics 2015; november.

74. Fecker LF, Stockfleth E, Nindl I, Ulrich C, Forschner T, Eberle J. The role of apoptosis in therapy and prophylaxis of epithelial tumours by nonsteroidal anti-inflammatory drugs (NSAIDs). The British journal of dermatology 2007;156 Suppl 3:25-33.

75. Barker N, Clevers H. Mining the Wnt pathway for cancer therapeutics. Nature reviews 2006;5:997-1014.

76. Cayirli M, Kose O, Demiriz M. Clinical, dermoscopic and immunohistochemical assessment of actinic keratoses and evaluation of the effectiveness of diclofenac therapy with immunohistochemical analysis. Archives of dermatological research 2013;305:389-95.

77. Kiiski V, de Vries E, Flohil SC, et al. Risk factors for single and multiple basal cell carcinomas. Archives of dermatology 2010;146:848-55.

78. Camp RL, Chung GG, Rimm DL. Automated subcellular localization and quantification of protein expression in tissue microarrays. Nature medicine 2002;8:1323-7.

79. Daniel K, Maria A, Amelie L, et al. Somatostatin receptor immunohistochemistry in neuroendocrine tumors: comparison between manual and automated evaluation. International journal of clinical and experimental pathology 2014;7:4971-80.

80. Taylor CR, Levenson RM. Quantification of immunohistochemistry--issues concerning methods, utility and semiquantitative assessment II. Histopathology 2006;49:411-24.

81. Rubin MA, Zerkowski MP, Camp RL, et al. Quantitative determination of expression of the prostate cancer protein alpha-methylacyl-CoA racemase using automated quantitative analysis (AQUA): a novel paradigm for automated and continuous biomarker measurements. The American journal of pathology 2004;164:83140.

82. Polley MY, Leung SC, McShane LM, et al. An international Ki-67 reproducibility study. Journal of the National Cancer Institute 2013;105:1897-906.

83. Matos LL, Stabenow E, Tavares MR, Ferraz AR, Capelozzi VL, Pinhal MA. Immunohistochemistry quantification by a digital computer-assisted method compared to semiquantitative analysis. Clinics (Sao Paulo, Brazil) 2006;61:417-24.

84. Yaffe MJ. Mammographic density. Measurement of mammographic density. Breast cancer research : BCR 2008;10:209.

85. Lobbes MB, Cleutjens JP, Lima Passos V, et al. Density is in the eye of the beholder: visual versus semi-automated assessment of breast density on standard mammograms. Insights into imaging 2012;3:91-9. 
86. Rippey JJ. Why classify basal cell carcinomas? Histopathology 1998;32:393-8.

87. Schon MP, Schon M. Imiquimod: mode of action. The British journal of dermatology 2007;157 Suppl 2:8-13.

88. Wolff F, Loipetzberger A, Gruber W, Esterbauer H, Aberger F, Frischauf AM. Imiquimod directly inhibits Hedgehog signalling by stimulating adenosine receptor/protein kinase A-mediated GLI phosphorylation. Oncogene 2013;32:5574-81.

89. Brechbiel J, Miller-Moslin K, Adjei AA. Crosstalk between hedgehog and other signaling pathways as a basis for combination therapies in cancer. Cancer treatment reviews 2014.

90. van Rijsingen MC, van Bon B, van der Wilt GJ, Lagro-Janssen AL, Gerritsen MJ. The current and future role of general practitioners in skin cancer care: an assessment of 268 general practitioners. The British journal of dermatology 2014;170:1366-8.

91. Reefman E BA, Kukutsch N, Bergman W. Huidige en toekomstige rol huidtherapeuten in huidoncologische zorg. Nederlands Tijdschrift voor Dermatologie en Venereologie 2015;25:341-2.

92. Sharpe HJ, Pau G, Dijkgraaf GJ, et al. Genomic analysis of smoothened inhibitor resistance in basal cell carcinoma. Cancer cell 2015;27:327-41.

93. Liu LS, Colegio OR. Molecularly targeted therapies for nonmelanoma skin cancers. International journal of dermatology 2013;52:654-65.

94. Axelson M, Liu K, Jiang X, et al. U.S. Food and Drug Administration approval: vismodegib for recurrent, locally advanced, or metastatic basal cell carcinoma. Clinical cancer research : an official journal of the American Association for Cancer Research 2013;19:2289-93.

95. Burness CB. Sonidegib: First Global Approval. Drugs 2015;75:1559-66.

96. Basset-Seguin N, Hauschild A, Grob JJ, et al. Vismodegib in patients with advanced basal cell carcinoma (STEVIE): a pre-planned interim analysis of an international, open-label trial. The lancet oncology 2015;16:729-36.

97. Basset-Seguin N, Sharpe HJ, de Sauvage FJ. Efficacy of Hedgehog Pathway Inhibitors in Basal Cell Carcinoma. Molecular cancer therapeutics 2015;14:633-41.

98. Chang AL, Atwood SX, Tartar DM, Oro AE. Surgical excision after neoadjuvant therapy with vismodegib for a locally advanced basal cell carcinoma and resistant basal carcinomas in Gorlin syndrome. JAMA dermatology 2013;149:639-41.

99. Chang AL, Oro AE. Initial assessment of tumor regrowth after vismodegib in advanced Basal cell carcinoma. Archives of dermatology 2012;148:1324-5.

100. Fecher LA. Systemic therapy for inoperable and metastatic basal cell cancer. Current treatment options in oncology 2013;14:237-48.

101. Madan V, Lear JT, Szeimies RM. Non-melanoma skin cancer. Lancet 2010;375:673-85.

102. Dijkgraaf GJ, Alicke B, Weinmann L, et al. Small molecule inhibition of GDC-0449 refractory smoothened mutants and downstream mechanisms of drug resistance. Cancer research 2011;71:435-44.

103. Pricl S, Cortelazzi B, Dal Col V, et al. Smoothened (SMO) receptor mutations dictate resistance to vismodegib in basal cell carcinoma. Molecular Oncology 2015;9:389-97.

104. Yauch RL, Dijkgraaf GJ, Alicke B, et al. Smoothened mutation confers resistance to a Hedgehog pathway inhibitor in medulloblastoma. Science 2009;326:572-4.

105. Flaherty KT, Infante JR, Daud A, et al. Combined BRAF and MEK inhibition in melanoma with BRAF V600 mutations. The New England journal of medicine 2012;367:1694-703.

106. Kim DJ, Kim J, Spaunhurst K, et al. Open-label, exploratory phase II trial of oral itraconazole for the treatment of basal cell carcinoma. Journal of clinical oncology : official journal of the American Society of Clinical Oncology 2014;32:745-51.

107. Chen AC, Martin AJ, Choy B, et al. A Phase 3 Randomized Trial of Nicotinamide for Skin-Cancer Chemoprevention. The New England journal of medicine 2015;373:1618-26. 
108. Iarrobino A, Messina JL, Kudchadkar R, Sondak VK. Emergence of a squamous cell carcinoma phenotype following treatment of metastatic basal cell carcinoma with vismodegib. Journal of the American Academy of Dermatology 2013;69:e33-4.

109. Saintes C, Saint-Jean M, Brocard A, et al. Development of squamous cell carcinoma into basal cell carcinoma under treatment with Vismodegib. Journal of the European Academy of Dermatology and Venereology : JEADV 2015;29:1006-9.

110. Orouji A, Goerdt S, Utikal J, Leverkus M. Multiple highly and moderately differentiated squamous cell carcinomas of the skin during vismodegib treatment of inoperable basal cell carcinoma. The British journal of dermatology 2014;171:431-3.

111. Aasi S, Silkiss R, Tang JY, et al. New onset of keratoacanthomas after vismodegib treatment for locally advanced basal cell carcinomas: a report of 2 cases. JAMA dermatology 2013;149:242-3.

112. Poulalhon N, Dalle S, Balme B, Thomas L. Fast-growing cutaneous squamous cell carcinoma in a patient treated with vismodegib. Dermatology 2015;230:101-4.

113. Zhu GA, Sundram U, Chang AL. Two different scenarios of squamous cell carcinoma within advanced Basal cell carcinomas: cases illustrating the importance of serial biopsy during vismodegib usage. JAMA dermatology 2014; 150:970-3.

114. Kakanj P, Reuter K, Sequaris G, et al. Indian hedgehog controls proliferation and differentiation in skin tumorigenesis and protects against malignant progression. Cell Reports 2013;4:340-51.

115. Ransohoff KJ, Tang JY, Sarin KY. Squamous Change in Basal-Cell Carcinoma with Drug Resistance. The New England journal of medicine 2015;373:1079-82.

116. Zhao X, Ponomaryov T, Ornell KJ, et al. RAS/MAPK Activation Drives Resistance to Smo Inhibition, Metastasis, and Tumor Evolution in Shh Pathway-Dependent Tumors. Cancer research 2015;75:3623-35.

117. Do H, Dobrovic A. Sequence artifacts in DNA from formalin-fixed tissues: causes and strategies for minimization. Clinical Chemistry 2015;61:64-71.

118. Hiatt JB, Pritchard CC, Salipante SJ, O'Roak BJ, Shendure J. Single molecule molecular inversion probes for targeted, high-accuracy detection of low-frequency variation. Genome Res 2013;23:843-54.

119. Leiter U HU, Gutzmer R, Haenssle H, Haefner HM, Mohr P, Garbe C. A phase II, single-armed, multicenter trial of neoadjuvant vismodegib in patients with large and/or recurrent basal cell carcinoma: NICCI. Journal of Clinical Oncology 2014;2014:suppl; abstr TPS9116.

120. Ally MS, Aasi S, Wysong A, et al. An investigator-initiated open-label clinical trial of vismodegib as a neoadjuvant to surgery for high-risk basal cell carcinoma. Journal of the American Academy of Dermatology 2014;71:904-11 e1.

121. Alcalay J, Tauber G, Fenig E, Hodak E. Vismodegib as a neoadjuvant treatment to Mohs surgery for aggressive basal cell carcinoma. Journal of drugs in dermatology : JDD 2015;14:219-23.

122. Tang T, Tang JY, Li D, et al. Targeting superficial or nodular Basal cell carcinoma with topically formulated small molecule inhibitor of smoothened. Clinical cancer research : an official journal of the American Association for Cancer Research 2011;17:3378-87.

123. Skvara H, Kalthoff F, Meingassner JG, et al. Topical treatment of Basal cell carcinomas in nevoid Basal cell carcinoma syndrome with a smoothened inhibitor. The Journal of investigative dermatology 2011;131:173544. 



\section{Chapter 7}

Summary/Samenvatting 


\section{Summary}

A general introduction to this thesis is given in chapter 1. This chapter provides a brief overview of the current knowledge on basal cell carcinoma (BCC), the most common cancer worldwide, with emphasis on BCC epidemiology, pathogenesis, clinical presentation, diagnosis and treatment. The content of this thesis is divided into two parts. Part one focuses on the pathogenesis of $\mathrm{BCC}$ and the occurrence of epigenetic modifications in particular. New topical and systemic treatments for both non-aggressive BCC (naBCC) and locally advanced BCC (laBCC) are evaluated en described in part two. Atthe end, the aims of this thesis are discussed.

The genetic background of BCC has been studied extensively, while its epigenetic makeup has received comparatively little attention. Epigenetic modifications are hereditary alterations by mechanisms other than changes in the DNA sequence, of which DNA methylation is the most widely studied. These epigenetic alterations are thought to occur at least as frequent as genetic mutations in the development of cancer by silencing of crucial tumor suppressor genes. We hypothesized that DNA-methylation might also contribute to BCC tumorigenesis and could even affect gene expression (part one).

In a first exploratory study (chapter 2.1), we analyzed the promoter methylation status of ten candidate (tumor suppressor) genes that are associated with Sonic Hedgehog $(\mathrm{SHH})$ signaling, WNT signaling and tumor growth. Methylation-specific PCR (MSP) was used in a large series of 112 BCCs and 124 healthy control samples. We found significantly more methylation in BCCs vs. healthy skin samples for the following gene promoters: $S H H$, adenomatous polyposis coli $(A P C)$, secreted frizzled-related protein 5 (SFRP5) and Ras association domain family $1 \mathrm{~A}$ (RASSF1A). As promoter methylation may result in gene silencing, we performed reverse transcription and quantitative reverse transcription PCR (RT-PCR), which showed reduced mRNA levels of these four genes for APC and SFRP5 in BCCs ( $n=6)$ vs. normal skin $(n=6)$. Significant down regulation of SHH, APC and RASSF1A could be confirmed on protein level as well by immunohistochemical staining. Increased canonical WNT activity was visualized by $\beta$-catenin staining, showing nuclear $\beta$-catenin in only $28 / 101(27.7 \%)$ of BCC. Absence of nuclear $\beta$-catenin in the majority of the samples may be due to high levels of membranous E-cadherin (in $94.1 \%$ of the samples). We detected no considerable changes in methylation status between sun-protected and sun-exposed skin, suggesting that the methylation pattern we detected is tumor, rather than locus or patient-specific. In conclusion, we provide evidence suggesting promoter hypermethylationmediated silencing of key players within the SHH and WNT pathways is frequent in BCCs, which is consistent with their known constitutive activation in BCCs. However, as BCCs are highly heterogeneous, it seems likely that aberrant epigenetic regulation of additional genes could contribute to BCCs development. With MSP it is possible to analyze predefined genes only. Therefore, in chapter 2.2, we performed genome-wide methylated DNA enrichment sequencing (MethylCap-seq) on 5 sporadic BCC and 5 healthy skin samples. These results were integrated with genome wide RNA-sequence data (RNA-seq henceforth), constituting the functional methylome. Fifteen differentially methylated or expressed loci in the BCC 
samples were identified, among which PTCH1 (part of SHH pathway), PTPRN2 (methylation marker in squamous lung cell carcinoma and IFI30 (playing a critical immunoregulatory role in melanoma). To further place the RNA expression patterns in a functional context, we performed pathway analysis of the RNA-seq data with a SPIA software tool. This in silico pathway analysis firmly implicated cytokine signaling, chemokine signaling and Toll-like receptor signaling in BCC growth and pathway activity, which we confirmed with immunohistochemical staining. Thus, we identified significant epigenetic modulation of gene expression in BCC and uncovered immunomodulatory signaling as a driver in BCC development.

In chapter 2.3 we compared the MethylCap-seq and RNA-seq data acquired in chapter 2.2 with those of a laBCC. This comparison provides the first evidence that there are substantial differences in gene methylation and expression levels between laBCC and naBCC. We found a significantly higher level of MMP13 expression in laBCC. This finding might explain why laBCC are much more aggressive than naBCC, because MMP13 is an enzyme that degrades extracellular matrix and bone. We also suggest that MMP13 might be used as a biomarker to distinguish BCC that are potentially aggressive and should be treated and followed up more rigorously to prevent local recurrence or evolution into a laBCC.

In our attempts to gain better insight into BCC pathogenesis, we realized that thinking 'out of the box' is crucial. As hair follicles are hypoxic microenvironments and BCCs are considered hair follicle tumors, we hypothesized that hypoxia-induced signaling pathways could be involved in BCCs as they are in other human malignancies. Hypoxia-inducible factor 1 (HIF1) and mechanistic/mammalian target of rapamycin (mTOR) are key players in the hypoxia pathways. In addition, the differentiation between BCCs and trichoepitheliomas (TEs), benign neoplasms that strongly resemble BCC, can be hard. Therefore we decided to compare these two tumors in chapter 3.1. We used immunohistochemical staining of formalin-fixed paraffin-embedded BCCs $(n=45)$ and TEs $(n=35)$ samples to assess activity of HIF1, mTORC1 and their most important target genes. Among the 45 BCCs and 35 TEs examined, most expression levels of several HIF1a and mTORC1 targets were comparable between BCCs and TEs. However, CAIX, Glut1 and PHD2 expression levels were significantly higher in TEs when only samples with at least $80 \%$ expression were included. From these results we concluded that HIF and mTORC1 signaling seems active in both BCCs and TEs. However, there are no appreciable differences between the two with respect to pathway activity. At this moment immunohistochemical analyses of HIF, mTORC1 and their target genes does not provide a reliable diagnostic tool for the discrimination of BCCs and TEs.

Current cancer research focuses ever more on the development of targeted treatments that specifically suppress key signaling pathways, as opposed to the more generally used nonspecific cytotoxic agents. Part two of this thesis provides novel insights in the usefulness of both topical and systemic targeted treatments in naBCCs and laBCCs respectively. 
Non-invasive treatments for low risk BCCs have several advantages over conventional surgical excision, such as lower healthcare costs, a decrease in the workload for dermatologists, a better cosmetic outcome and a higher patient preference. Photodynamic therapy (PDT) (photochemical), imiquimod (immune-modulating) and 5-fluorouracil cream (chemotherapeutic) are available non-invasive treatments for low risk BCC that are well studied and already frequently prescribed. Apart from imiquimod, however, they all seem to have a mode of action of targeting non-specific signaling pathways activated in BCC. We hypothesized that higher efficacy might be expected when signaling pathways activated in BCC are specifically targeted, herewith potentially reaching efficacy rates of surgical excision. Non-steroidal anti-inflammatory drugs (NSAIDs) and vitamin D derivatives such as calcitriol can interfere in the SHH and WNT pathways, as well as in apoptosis signaling. From this, we hypothesized in chapter $\mathbf{4 . 1}$ that topically applied diclofenac sodium $3 \%$ gel (Solaraze $\AA$ ), calcitriol $3 \mu \mathrm{g} / \mathrm{g}$ ointment (Silkis $\AA$ ) and a combination of both would result in a decrease in proliferation and an increase in apoptosis in superficial (sBCCs) and nodular (nBCCs). In a phase II, single blind, randomized controlled intervention trial, patients with a primary, histologically proven $\mathrm{sBCC}(n=64)$ or $\mathrm{nBCC}(n=64)$ were randomly assigned to receive either topical diclofenac, calcitriol, a combination of both or no topical treatment (control group). For eight weeks, patients applied the vehicle twice a day and covered it with an occlusive sheet. At the end of the treatment period, all tumors were excised and histologically examined. The primary outcome measure was the post-treatment expression of Ki-67 (proliferation marker) and Bcl-2 (apoptosis marker). Histological clearance, adverse events, application-site reactions, patient compliance and patient satisfaction were secondary outcomes. We were able to confirm a significant decrease in Ki-67 and $\mathrm{Bcl}-2$ expression in sBCCs treated with diclofenac and for Ki-67 after combination therapy. In addition, we found complete histological tumor regression in $64.3 \%$ of sBCCs after diclofenac and $43.8 \%$ of sBCCs after combination therapy, compared to $0.0 \%$ of controls. We found no tumor regression in nBCCs, and no appreciable changes in either Ki-67 or $\mathrm{Bcl}-2$ expression levels, probably due to insufficient penetration of the drugs at the tumor site. Application-site reactions were reported frequently in the diclofenac groups, though most reactions were of mild to moderate severity and similar as seen in other non-invasive therapies for sBCCs, such as imiquimod and 5-fluorouracil cream. Repetitive occlusion as used in our trial may have enhanced the severity of the skin reactions. Despite the side effects, the compliance was high, ranging from $91.82 \%$ to $98.18 \%$. From our results we conclude that, although surgical excision remains the gold standard for all BCCs, topical diclofenac may be a promising new treatment for low risk sBCCs. Because of its different mode of action by targeting several molecular pathways activated in BCCs, the expected major benefit of diclofenac gel is that is effective in BCC's non-responsive to non-specific or $\mathrm{SHH}$-targeting agents. Additionally, as simultaneously targeting $\mathrm{SHH}$ and other signaling pathways may have a synergistic effect, we speculate that combining diclofenac with imiquimod (currently the most effective non-invasive treatment in SBCCs) might be promising. 
Immunohistochemical (IHC) analysis as assessed in chapter $\mathbf{4 . 1}$ is being used more often to gain insight in the underlying mode of action or efficacy of new, targeted therapies for BCCs. Manual pathologist-based quantification (MQ) is still the gold standard in the assessment of IHC slides, however, it is time-consuming and labor-intensive as well as subject to high intraand inter-observer variability. Digital image analysis techniques offer an objective approach with a high sensitivity for change and are expected to have an increased reproducibility. However, comparative studies that test the assumption of high reproducibility of digital BCC image analyses are lacking. In chapter 4.2 we used semi-automated quantification (SAQ) for the evaluation of $122 \mathrm{sBCCs}$ and $\mathrm{nBCC}$ and found reasonable high inter-observer agreements with $95 \%$ limits of agreement between $-9.2 \%$ and $10.8 \%$ for $\mathrm{Ki}-67$ and between $-12.2 \%$ and $20.0 \%$ for Bcl-2. Two trained, but previously inexperienced, investigators performed SAQ independently. However, large discrepancies were seen between SAQ and $M Q$ (performed by one experienced dermato-pathologist), especially at higher expression levels (more than $20 \%$ positive tumor cells) of $\mathrm{Ki}-67$ and $\mathrm{Bcl}-2$, which could partly be explained by systematic error. Our findings point to the need for a further study wherein consensus evaluation by more than one pathologist will be compared with SAQ measurements.

When naBCC are neglected or inadequately treated, they have the potential to develop into a laBCC. In 2012, the U.S Food and drug administration (FDA) approved vismodegib, an orally active synthetic SMO inhibitor, for the treatment of laBCC and $\mathrm{mBCC}$. However, primary (no initial response to treatment) or secondary/acquired resistance (progression after an initial response) are emerging problems. Chapter $\mathbf{5 . 1}$ describes a 68-year old woman with a laBCC of the left shoulder who developed tumor regrowth 20 weeks after an initially good response to vismodegib. Mutation analysis of PTCH1 and SMO was performed on primary tumor tissue as well as on clinically responsive skin and tumors that arose during treatment. PTCH1 mutations were found in the primary tumor and in two newly developed tumor nodules, while they were absent in clinically and histologically responding tissue. This finding implies that cells from the original tumor had survived. Additionally, the recurrent tumor nodules harbored two SMO mutations (p.Trp281Leu and p.Val321Met) which were neither found in the primary $\mathrm{BCC}$ nor in the responding skin. Thus, even though the resistant tumors arose from the same, primary tumor, they were not clonal. Both SMO mutations may probably cause resistance for $\mathrm{SMO}$ inhibitors. Currently, classification of resistance as being primary or secondary is based on clinical characteristics. A patient with presumably primary resistance to vismodegib is discussed in chapter 5.2. A 52-year old man was treated with vismodegib for a recurrent $\mathrm{BCC}$ of the nose and right cheek, that had previously been inadequately treated. After 7 weeks of treatment, hyperkeratosis had increased and comedo-like lesions had developed on the nose. No improvement was observed and a transition into a squamous cell carcinoma (SCC) was feared. The Sequential skin biopsies showed epidermal cysts, but no residual BCC or newly formed SCC. Because of the possibility of a sample error, Mohs' micrographic surgery was performed, showing infiltrative BCC but no SCC, which was confirmed by immunohistochemical stains for Ber-Ep4. Formation of pseudocyst-like structures and SCC within the initial BCC following vismodegib treatment has been reported previously in limited cases only. Also, the development of SCC and keratoacanthoma on other body 
sites has been described in literature. It is therefore important to be aware of the possibility of occurrence of SCC during vismodegib treatment. Sequential mutation analysis on pretreatment and recurrent tumor tissue may be helpful to anticipate on the resistance (either primary or secondary) in order to actively alter therapy. Accordingly, treatment schedules and combination therapies can be customized to both patient and tumor characteristics. 


\section{Samenvatting}

In het eerste hoofdstuk wordt een algemene inleiding tot dit proefschrift gegeven. Het hoofdstuk schetst in het kort de huidige kennis van het basaalcelcarcinoom (BCC), wereldwijd de meest voorkomende vorm van kanker. Denadruk ligt hierbij op epidemiologie, pathogenese, klinische presentatie, diagnose en behandeling. Inhoudelijk bestaat dit proefschrift uit twee delen. Het eerste deel is gericht op de pathogenese van BCC, en dan met name op de epigenetische modificaties. In het tweede deel worden nieuwe topicale en systemische behandelingen voor zowel niet-agressieve (naBCC) als lokaal agressieve BCC (laBCC) besproken en geëvalueerd. Tot slot worden de doelstellingen van dit proefschrift beschreven.

De genetische achtergrond van BCC is uitvoerig onderzocht, maar aan de epigenetische achtergrond is verhoudingsgewijs minder aandacht besteed. Epigenetische modificaties zijn erfelijk bepaald door andere mechanismen dan veranderingen in de DNA sequentie, waarvan DNA methylering het meest onderzocht is. Verondersteld wordt dat deze epigenetische veranderingen minstens zo vaak optreden bij de ontwikkeling van kanker als genetische mutaties, dit door inactivatie van cruciale tumorsuppressorgenen. Onze hypothese is dat DNA-methylering mogelijk ook bijdraagt aan de tumorgenese van het BCC en zelfs de genexpressie kan beïnvloeden (deel 1).

In een eerste experimentele studie (hoofdstuk 2.1) hebben we de promotor methyleringsstatus van tien kandidaat (tumorsuppressor) genen geanalyseerd. Deze genen zijn geassocieerd met de Sonic Hedgehog $(\mathrm{SHH})$ signalering, de WNT signaaltranductieroute en tumorgroei. Er is gebruikgemaakt van methylering-specifieke PCR (MSP) bij de analyse van 112 BCC's en 124 gezonde controlemonsters. In de volgende promotorgenen is er significant meer methylering gevonden in BCC's dan in de gezonde controlegroep: $S H H$, adenomateuze polyposis coli $(A P C)$, gesecreteerde frizzled gerelateerd eiwit 5 (SFRP5) en Ras geassocieerde domein familie 1A (RASSF1A). We hebben een reverse transcriptie en kwantitatieve reverse transcriptie PCR (RT-PCR) uitgevoerd omdat methylering van een promotor kan leiden tot inactivatie van een gen. Van de hiervoor genoemde vier genen werd er in APC en SFRP5 een verminderde concentratie mRNA in BCC's ( $n=6)$ gezien ten opzichte van normale huid $(n=6)$. Een significante downregulatie van SHH, APC en RASSF1A zou bevestigd kunnen worden zowel op eiwitniveau als door middel van immunohistochemische kleuringen. Door aankleuring van $\beta$-catenine werd in slechts $28 / 101(27,7 \%)$ van de BCC's verhoogde canonicale WNT-activiteit zichtbaar. Nucleair $\beta$-catenine was in de meerderheid van de samples afwezig; dit kan te verklaren zijn door de hoge concentraties membraneus E-cadherine (94,1\% van de samples). We hebben geen noemenswaardige veranderingen in het methyleringspatroon tussen de aan zon blootgestelde huid en de voor zon beschermde huid gevonden. Dit suggereert dat het vastgestelde methyleringspatroon eerder bij een tumor past dan dat het locus- en patiëntspecifiek is. We leveren dus bewijs aan dat doet vermoeden dat inactivatie van hoofdrolspelers in de SHH en WNT signaaltransductieroutes door middel van hypermethylering van een promotor frequent voorkomt in BCC's. Dit komt overeen met de reeds bekende constitutieve activatie van deze signaaltransductieroutes in 
BCC's. BCC's zijn echter zeer heterogeen, waardoor het waarschijnlijk lijkt dat een abnormale epigenetische regulatie van additionele genen kan bijdragen aan de ontwikkeling van BCC's. Met behulp van MSP is het alleen mogelijk om vooraf bepaalde genen te analyseren. In hoofdstuk 2.2 hebben we dan ook gekeken naar genoomwijde 'methylated DNA enrichment sequencing' (MethylCap-seq) op vijf sporadische BCC's en op vijf gezonde huidmonsters. Deze resultaten zijn geïntegreerd met genoomwijde RNA-sequentie data (RNA-seq) die het functionele methyloom vormen. Er zijn vijftien differentieel gemethyleerde of tot expressie gebrachte loci in de BCC-monsters geïdentificeerd, waaronder PTCH1 (deel van de SHH signaaltransductie), PTPRN2 (methylering marker in plaveiselcelcarcinoom van de long) en IFI30 (speelt een cruciale rol in de immunoregulatie van melanomen). Om de RNA expressiepatronen in een functionele context te kunnen plaatsen, hebben we met de SPIA software de signaaltransductieroutes van de RNA-seq data geanalyseerd. Deze in silico signaaltransductierouteanalyse liet duidelijk cytokine signalering, chemokine signalering en Toll-like receptor signalering zien in BCC-groei en signaaltransductieactiviteit. Dit laatste hebben wij door middel van immunohistochemische kleuringen bevestigd. We hebben dus significante epigenetische modulatie van genexpressie in BCC geïdentificeerd en ontdekt dat de immunomodulerende signalering een aanjager is in de ontwikkeling van BCC's.

In hoofdstuk 2.3 hebben we de verkregen MethylCap-seq en RNA-seq data uit hoofdstuk 2.2 vergeleken met die van een laBCC. Deze vergelijking levert het eerste bewijs dat er substantieel verschil is in de hoeveelheid gen methylering en expressie tussen IaBCC en naBCC. We hebben een significant hogere concentratie MMP13 expressie gevonden in laBCC. Deze bevinding zou kunnen verklaren waarom laBCC een veel agressievere vorm is dan naBCC. MMP13 is namelijk een enzym dat de extracellulaire matrix en het bot afbreekt. Wij suggereren dat MMP13 als biomarker gebruikt zou kunnen worden om in potentie agressieve BCC's te herkennen. Op deze BCC's zouden dan een rigoureuzere behandeling en follow-up kunnen worden toegepast om een lokaal recidief of de ontwikkeling tot een laBCC te voorkomen.

Bij onze pogingen om meer inzicht te krijgen in de pathogenese van het BCC hebben we ons gerealiseerd dat 'out of the box' denken van cruciaal belang is. Een haarfollikel is een hypoxisch micromilieu en er wordt gedacht dat BCC's haarfollikeltumoren zijn. Onze hypothese is dan ook dat door hypoxie geïnduceerde signaaltransductieroutes een rol kunnen spelen bij BCC's. De activiteit van deze signaaltransductieroutes wordt ook in andere humane carcinomen gezien. Hypoxia-inducible factor 1 (HIF-1) en mechanistic/mammalian target of rapamycin (mTOR) zijn hoofdrolspelers in de hypoxische signaaltransductieroutes. Het onderscheid tussen BCC en een trichoepithelioom (TE), een goedaardige tumor die veel op het BCC lijkt, kan moeilijk zijn. In hoofdstuk $\mathbf{3 . 1}$ hebben we daarom deze twee tumoren met elkaar vergeleken. We hebben immunohistochemische kleuringen gebruikt om de activiteit van HIF1, mTORC1 en hun belangrijkste doelgenen aan te tonen op formaline gefixeerde paraffine preparaten van BCC $(n=45)$ en TE $(n=35)$. De meeste expressieniveaus van de verschillende HIF1a en mTORC1 targets zijn vergelijkbaar tussen de onderzochte 45 BCC's en 35 TE's. Als alleen samples met een expressie van 
tenminste $80 \%$ worden meegerekend, is in de TE's de expressie van CAIX, Glut1 en PHD2 significant hoger. Hieruit kunnen we concluderen dat HIF en mTORC1 signalering in zowel BCC's als TE's actief is. Er zijn echter geen noemenswaardige verschillen gevonden tussen de twee groepen ten aanzien van de activiteit van de signaaltransductieroute. Op dit moment lijken de immunohistochemische analyses van HIF, mTORC1 en hun doelgenen geen zinvol diagnosticum voor het onderscheid tussen BCC 's en TE's.

De huidige onderzoeken naar kanker richten zich steeds meer op de ontwikkeling van doelgerichte behandelingen, die specifieke hoofdrolspelers van een signaaltransductieroute onderdrukken, in tegenstelling tot de huidige meer generieke en niet-specifieke cytotoxische middelen. In deel 2 van dit proefschrift wordt nader ingegaan op de bruikbaarheid van zowel systemische als topicale doelgerichte behandelingen in respectievelijk naBCC en laBCC.

Non-invasieve behandelmethoden voor BCC's met een laag risico hebben verschillende voordelen boven conventionele chirurgische excisie: lagere kosten, daling van de werkdruk voor dermatologen, een beter cosmetisch resultaat en een hogere patiënttevredenheid. Photodynamische therapie (PDT) (fotochemisch), imiquimod (immuunmodulerend) en 5-flourouracil crème (cytostaticum) zijn goed onderzochte en reeds vaak voorgeschreven non-invasieve behandelopties voor weinig risicovolle BCC's. Op imiquimod na, lijken ze allemaal aan te grijpen op niet-specifieke signaaltransductieroutes die geactiveerd zijn in BCC. Onze hypothese is dat er een hogere effectiviteit van topicale behandelingen bereikt kan worden, als de topicale middelen specifiek op de geactiveerde signaaltransductieroutes van BCC aangrijpen. Op deze manier kan mogelijk de effectiviteit van chirurgische ingreep worden benaderd. Niet-steroïde ontstekingsremmers (NSAID's) en vitamine D-derivaten, zoals calcitriol, kunnen interfereren met de SHH, WNT en apoptose signaaltransductieroutes. Op basis hiervan is onze hypothese in hoofdstuk 4.1 dat topicale diclofenac sodium $3 \%$ gel (Solaraze $\AA$ ), calcitriol $3 \mu \mathrm{g} / \mathrm{g}$ crème (Silkis $\AA$ ) en een combinatie van beide leiden tot een daling van de proliferatie en een stijging van de apoptose in superficiële BCC (sBCC) en nodulaire BCC ( $\mathrm{nBCC}$ ). In een fase II, geblindeerd, gerandomiseerd en gecontroleerd onderzoek werden patiënten met een primair, histologisch bevestigd sBCC $(n=64)$ of $n B C C \quad(n=64)$ gerandomiseerd in ofwel topicale diclofenac, calcitriol of een combinatie van beide ofwel geen topicale behandeling (controlegroep). Patiënten hebben gedurende acht weken tweemaal daags de crème aangebracht en vervolgens bedekt met afsluitende folie. Aan het eind van de behandelperiode zijn alle tumoren geëxcideerd en histologisch onderzocht. De primaire uitkomstmaat was de meting van de expressie van Ki-67 (proliferatiemarker) en Bcl-2 (apoptose marker) na de behandeling. Histologische remissie, bijwerkingen, lokale huidreacties, compliantie en patiënttevredenheid waren secundaire uitkomstmaten. We hebben een significante daling van Ki-67 en Bcl-2 expressie gevonden in sBCC's die behandeld werden met diclofenac. Bij patiënten die behandeld zijn met de combinatietherapie is een significante daling van Ki-67 gevonden. Verder werd volledige histologische regressie van de sBCC's in $64,3 \%$ en $43,8 \%$ van de patiënten die behandeld werden met respectievelijk diclofenac en de combinatietherapie tegen $0,0 \%$ in de controlegroep. Bij de nBCC's hebben we geen tumorregressie en ook geen 
noemenswaardige veranderingen in de $\mathrm{Ki}-67$ of $\mathrm{Bcl}-2$ expressie gevonden. Dit is mogelijk te verklaren door onvoldoende penetratie van de medicijnen op de plek van de tumor. Lokale huidreacties kwamen frequent voor in de groepen met diclofenac. De meeste reacties waren mild tot matig van ernst en vergelijkbaar met die van andere non-invasieve behandelingen voor sBCC's, zoals imiquimod en 5-fluorourcil creme. De herhaaldelijke occlusie zoals wij in onze trial hebben gebruikt, heeft mogelijk tot heftiger huidreacties geleid. Ondanks de bijwerkingen was de compliantie hoog, tussen $91,82 \%$ en $98,18 \%$. Uit onze resultaten kunnen we concluderen dat, ondanks het feit dat chirurgische excisie voor alle BCC's de gouden standaard blijt, topicale diclofenac een veelbelovende nieuwe behandelmethode kan zijn voor laag risico sBCC's. Diclofenac gel heeft door het andere werkingsmechanisme effect op verschillende in BCC's geactiveerde moleculaire signaaltransductieroutes. Het grote voordeel dat we hiervan verwachten, is dat diclofenac juist effectief is in BCC's die niet reageren op de niet-specifieke behandelingen of de behandelingen die alleen op de $\mathrm{SHH}$-signaaltransductie aangrijpen. Als bij een behandeling van BCC's zowel de $\mathrm{SHH}$ als andere signaaltransductieroutes het doel zijn, kan dit een synergetisch effect hebben. We speculeren dan ook dat de combinatie van diclofenac met imiquimod (op dit moment de meest effectieve vorm van non-invasieve behandeling in sBCC's) veelbelovend kan zijn.

De immunohistochemische kleuringen (IHK) die in hoofdstuk 4.1 zijn beoordeeld, worden gebruikt om meer inzicht te krijgen in het onderliggende werkingsmechanisme en de effectiviteit van nieuwe, doelgerichte behandelopties in BCC's. Handmatige kwantificatie (HK) door een patholoog wordt voor de beoordeling van IHK nog steeds als gouden standaard gezien. Dit is echter tijdrovend en arbeidsintensief en heeft een hoge intra- en interobserver variabiliteit. Digitale coupe analysetechnieken bieden een objectieve benadering met hoge sensitiviteit voor verandering en hebben waarschijnlijk een hogere reproduceerbaarheid. Er zijn nog geen vergelijkende studies bekend die deze verwachte reproduceerbaarheid van digitale coupeanalyses voor BCC's bevestigen. In hoofdstuk $\mathbf{4 . 2}$ hebben we 122 sBCC's en nBCC's beoordeeld door middel van semi-automatische kwantificatie (SAK). Hierbij hebben we toereikend hoge interobserver overeenstemming gevonden met 95\%-betrouwbaarheidsintervallen tussen -9,2\% en 10,8\% voor Ki-67 en tussen -12,2\% en $20,0 \%$ voor Bcl-2. De SAK is onafhankelijk uitgevoerd door twee daartoe opgeleide maar nog onervaren onderzoekers. Hierbij zijn grote discrepanties gezien tussen de SAK en HK (verricht door één ervaren dermato-patholoog), met name bij hogere expressie van Ki-67 en $\mathrm{Bcl}-2$ (meer dan 20\% van de tumorcellen is positief). Dit is gedeeltelijk te verklaren door een systematische fout. Onze bevindingen geven aanleiding voor verder onderzoek waarin de $\mathrm{HK}$, die door meer dan één wordt verricht, wordt vergeleken met de uitkomsten van de SAK.

Een naBCC heeft de potentie om zich te ontwikkelen tot een laBCC indien niet of onvoldoende behandeld. In 2012 heeft de Amerikaanse 'Food and drug administration' (FDA) vismodegib, een oraal actieve synthetische SMO inhibitor, goedgekeurd voor de behandeling van laBCC en gemetastaseerd BCC (mBCC). Primaire (geen reactie op de behandeling) en secundaire/ verkregen (progressie na initiele respons) resistentie is bij deze behandeling een probleem. In hoofdstuk 5.1 wordt een casus beschreven van een 68-jarige vrouw met een laBCC op de 
linkerschouder. De tumor reageerde initieel goed op vismodegib maar na 20 weken begon deze opnieuw te groeien. Er zijn mutatieanalyses verricht van PTCH1 en SMO op de primaire tumor, de klinisch reagerende huid en de gedurende de behandeling groeiende tumoren. De PTCH1 mutaties werden gevonden in de primaire tumor en in de twee nieuw ontwikkelde tumornoduli, terwijl de mutatie afwezig was in de klinisch en histologisch reagerende huid. Dit impliceert dat tumorcellen van de oorspronkelijke tumor de behandeling overleefd hebben. Daarnaast werden bij de twee recidief noduli twee SMO mutaties (p.Trp281Leu en p.Val321Met) gevonden. Deze mutaties waren niet terug te vinden bij de primaire tumor, noch bij het gereageerde weefsel. Hoewel de resistente tumoren vanuit de primaire tumor groeiden, waren ze er dus geen klonen van. Beide SMO mutaties zouden de oorzaak kunnen zijn voor de resistentie voor de SMO inhibitors. Op dit moment is de classificatie primaire of secundaire resistentie gebaseerd op het klinische beeld. In hoofdstuk $\mathbf{5 . 2}$ wordt een patiënt besproken met vermoedelijk primaire resistentie voor vismodegib. Bij een 52-jarige man werd een recidief BCC van de neus en rechterwang behandeld met vismodegib, nadat het eerder inadequaat behandeld was. Na een behandelperiode van zeven weken was er een toename van de hyperkeratose te zien en ontwikkelde er zich verruceuze afwijkingen op de neus. Ondanks de behandeling werd er geen verbetering gezien en de angst bestond dat de tumor zich had ontwikkeld tot een plaveiselcelcarcinoom (PCC). In de daaropvolgende biopsieën werden epidermale cysten gezien, maar er waren geen aanwijzingen voor een recidief BCC of nieuw ontwikkeld PCC. Gezien de kans op een steekproeffout werd besloten Mohs' micrografische chirurgie te verrichten. Dit liet een infiltratief BCC maar geen PCC zien. Dit werd door middel van immunohistochemische kleuringen van Ber-Ep4 bevestigd. De ontwikkeling van pseudocysten en PCC binnen het initiële BCC bij behandeling met vismodegib is eerder in slechts een beperkt aantal casus besprekingen beschreven. De ontwikkeling van PCC en keratoacanthomen op andere locaties van het lichaam is ook in de literatuur beschreven. Het is dus belangrijk om alert te zijn op de mogelijke ontwikkeling van een PCC tijdens de behandeling met vismodegib. Sequentiële mutatieanalyses op onbehandeld en recidief tumorweefsel kan zinvol zijn om te anticiperen op resistentie (primair of secundair) en actief de behandeling hierop aan te passen. Op deze manier kunnen behandelschema's en combinatietherapieën worden toegespitst op zowel de patiënt als de tumorkenmerken. 



\section{Chapter 8}

Curriculum vitae

Grants and awards

List of publications

Oral \& poster presentations

Dankwoord 


\section{Curriculum vitae}

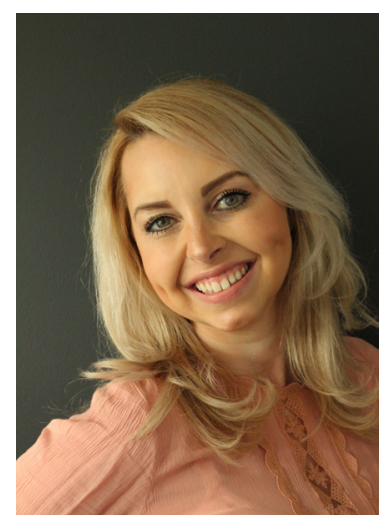

Tjinta Brinkhuizen werd geboren op 15 november 1982 in Maastricht. Hier groeide ze samen met haar zusje en haar ouders op. In 2002 behaalde ze haar gymnasium diploma aan het Trichter College te Maastricht, waarna ze startte met de opleiding moleculaire levenswetenschappen (nu biomedische wetenschappen) aan de transnationale Universiteit Limburg, dit omdat ze was uitgeloot voor geneeskunde. In 2003 werd ze alsnog ingeloot en startte ze met de opleiding geneeskunde. Tijdens de co-schappen ontstond haar passie voor de dermatologie. Deze werd verder bevestigd gedurende haar semi-arts stage in het laatste jaar van haar opleiding tot basisarts aan de afdeling dermatologie van het Maastricht Universitair Maastricht Centrum (MUMC). In dit laatste jaar werd ook de interesse voor wetensschappelijk onderzoek gewekt. Zij schreef een onderzoeksvoorstel voor een persoonsgebonden beurs van KWF kankerbestrijding, welke werd toegekend. Hierdoor kon ze aansluitend aan het behalen van haar artsexamen in oktober 2009 beginnen als arts-onderzoeker op de afdeling dermatologie van het MUMC. Onder begeleiding van promotoren prof.dr.M.A.M. van Steensel en prof.dr.P.M. Steijlen en co-promotor mevr.dr.K. Mosterd werkte zij aan verschillende studies op het gebied van epigenetische modificaties en nieuwe behandelingsmodaliteiten van het basaalcelcarcinoom, leidend tot deze dissertatie. Daarnaast is ze betrokken geweest bij studies naar het Birt-Hogg-Dubé syndroom en infantiele hemangiomen. In oktober 2012 is zij met veel enthousiasme gestart met de opleiding tot dermatoloog en combineert ze haar klinische taken met haar onderzoekswerkzaamheden. Medio 2017 zal zij deze opleiding afronden. Met haar onderzoek won ze in 2010 de prijs voor beste voordracht op het dermato-oncologiecongres in Spa (België). In oktober 2014 won ze de eerste prijs op het Pélerin wetenschapssymposium (MUMC), waarvoor ze eerder in 2012 ook genomineerd was. In 2013 won ze de NVDV reisbeurs voor het IID congres in Edinburgh, Schotland. 


\section{Grants and Awards}

Grant

Personal research grant Dutch Cancer Society (KWF):

Sum €243.000,-

Awards

October 2014

Winner Pélerin scientific award, MUMC, Maastricht, the Netherlands

Oral presentation: Topical diclofenac 3\%, vitamin D or a combination of both as treatment for basal cell carcinoma.

Reward €5000,-

January 2013

Travel grant for IID 2013 by Dutch Society for Dermatology and Venereology.

Reward €750,-

October 2012

Pélerin scientific award, MUMC, Maastricht, the Netherlands

Oral presentation: Basal cell carcinoma 'out of the box'.

Project selected for oral presentation, €1000,-

June 2010

Dermato-oncology congress, Spa, Belgium

Oral presentation: Basal cell carcinoma, Genetics, Epigenetics and future possible treatments.

Best presenting resident Dermatology, €500,- 


\section{List of publications}

Brinkhuizen T, Frencken KJA, Nelemans PJ, Hoff MLS, Kelleners-Smeets NWJ, zur Hausen A, van der Horst MPJ, Rennspiess D, Winnepenninckx WJL, van Steensel MAM, Mosterd K. The effect of topical diclofenac 3\% and calcitriol $3 \mu \mathrm{g} / \mathrm{g}$ on superficial basal cell carcinoma ( $S B C C)$ and nodular basal cell carcinoma (nBCC): A phase II, randomized controlled trial. J Am Acad Dermatol. 2016 April.

Brinkhuizen T, van Geel M, Denil SLIJ, de Meyer T, Kelleners-Smeets NWJ, Lohuis PJFM, Hoebers FJP, Winnepenninckx VJL, van Steensel MAM, Mosterd K. Locally advanced basal cell carcinoma has a distinct methylation and transcriptomic profile. Exp Dermat. 2016 Apr;25(4):316-8.

Frencken $\mathrm{KJ}^{\star}$, Hacking $\mathrm{MN}^{\star}$, Brinkhuizen T, Abdul Hamid MA, Martens H. Soft yellowish papules on the neck: a clinicopathological challenge. Clin Exp Dermatol. 2016 Mar;41(2):218-20.

Janmohammed SR, Brinkhuizen T, den Hollander JC, Madern GC, de Laat PC, van Steensel MA, Oranje AP. Support for the hypoxia theory in the pathogenesis of infantile haemangioma. Clin Exp Dermatol. 2015 Jun;40(4):431-7.

Reinders $\mathrm{MG}^{*}$, Brinkhuizen $\mathrm{T}^{*}$, Soetekouw PM, Kelleners-Smeets NW, Abdul Hamid MA, Mosterd K. Epidermal Cyst Formation and Hyperkeratosis in a Patient Treated with Vismodegib for Locally Advanced Basal Cell Carcinoma. Acta Derm Venereol. 2015 Apr 28;95(5):618619.

Brinkhuizen $\mathbf{T}^{\star}$, Reinders $\mathrm{MG}^{*}$, van Geel M, Hendriksen AJ, Paulussen AD, Winnepenninckx VJ, Keymeulen KB, Soetekouw PM, van Steensel MA, Mosterd K. Acquired resistance to the Hedgehog pathway inhibitor vismodegib due to smoothened mutations in treatment of locally advanced basal cell carcinoma. J Am Acad Dermatol. 2014 Nov;71(5):1005-8.

Brinkhuizen T, Weijzen CA, Eben J, Thissen MR, van Marion AM, Lohman BG, Winnepenninckx VJ, Nelemans PJ, van Steensel MA. Immunohistochemical analysis of the mechanistic target of rapamycin and hypoxia signalling pathways in basal cell carcinoma and trichoepithelioma. PLoS One. 2014 Sep 2;9(9):e106427.

Brinkhuizen T, van den Hurk K, Winnepenninckx V, de Hoon J, van Marion A, Veeck J, van Engeland M, van Steensel M. Epigenetic changes in Basal Cell Carcinoma affect SHH and WNT signalling components. PlosOne 2012 2012;7(12):e51710. 
Preston RS, Philp A, Claessens T, Gijezen L, Dydensborg AB, Dunlop EA, Harper KT, Brinkhuizen T, Menko FH, Davies DM, Land SC, Pause A, Baar K, van Steensel MA, Tee AR. Absence of the Birt-Hogg-Dubé gene product is associated with increased Hypoxia Inducible Factor transcriptional activity and a loss of metabolic flexibility. Oncogene 2010. 2011 Mar 10;30(10):1159-73.

Boonen A, Brinkhuizen T, Landewé R, van der Heijde T, Severens J. Impact of ankylosing spondylitis on sick-leave, presenteeism and unpaid productivity, and estimation of the societal cost. Ann Rheum Dis 2010. Jun;69(6):1123-8.

Dahlqvist J, Klar J, Tiwari N, Schuster J, Törmä H, Badhai J, Pujol R, van Steensel MA, Brinkhuizen T, Gijezen L, Chaves A, Tadini G, Vahlquist A, Dahl N. A single-nucleotide deletion in the POMP 5' UTR causes a transcriptional switch and altered epidermal proteasome distribution in KLICK genodermatosis. Am J Hum Genet. 2010 Apr 9;86(4):596-603.

van Boxem K, van Eerd M, Brinkhuizen T, Patijn J, van Kleef M, van Zundert J. Radiofrequency and pulsed radiofrequency treatment of chronic pain syndromes: the available evidence. Pain Pract 2008 Sep-Oct;8(5):385-93. 


\section{Oral presentations on conferences}

The effect of topical diclofenac 3\% and calcitriol $3 \mu \mathrm{g} / \mathrm{g}$ on superficial and nodular basal cell carcinoma: a phase II, randomized controlled trial.

$11^{\text {th }}$ European Association of Dermato Oncology, Marseille, France, October 2015.

Acquired resistance to the Hedgehog pathway inhibitor vismodegib due to smoothened mutations in treatment of locally advanced basal cell carcinoma.

16th Annual scientific meeting Dutch Association for Experimental Dermatology, Lunteren, the Netherlands, February 2015.

Topical diclofenac 3\%, vitamin D or a combination of both as treatment for basal cell carcinoma.

Pélerin scientific meeting (MUMC), Maastricht, the Netherlands, October 2014.

The functional methylome of Basal Cell Carcinoma and beyond.

Singapore International Conference on Skin Research, Biopolis, Singapore, March 2014

Topical diclofenac 3\%, vitamin D or a combination of both as treatment for basal cell carcinoma.

$15^{\text {th }}$ Annual scientific meeting Dutch Association for Experimental Dermatology, Lunteren, the Netherlands, January 2014.

Basal Cell Carcinoma 'Out of the Box'.

Pélerin scientific meeting (MUMC), Maastricht, the Netherlands, October 2012.

An exceptional mutilating basal cell carcinoma.

Annual scientific meeting Dutch Association for Dermatology and Venereology, Maastricht, the Netherlands, June 2012.

Basal Cell Carcinoma, Genetics, Epigenetics and future possible treatments.

World Congress of Dermatology, Seoul, South Korea, May 2011.

Basal Cell Carcinoma, Genetics, Epigenetics and future possible treatments.

$12^{\text {th }}$ Annual scientific meeting Dutch Association for Experimental Dermatology, Lunteren, the Netherlands, January 2011.

SMO inhibitors and Basal Cell Carcinoma.

Scientific meeting Dermatology departments 'Limburg', Maastricht, the Netherlands, November 2010.

Basal Cell Carcinoma, Genetics, Epigenetics and future possible treatments.

$2^{\text {nd }}$ World Congress on Genodermatology, Maastricht, the Netherlands, November 2010. 
Basal Cell Carcinoma, Genetics, Epigenetics and future possible treatments.

European Academy of Dermatology and Venereology, Gothenburg, Sweden, October 2010.

Basal Cell Carcinoma, Genetics, Epigenetics and future possible treatments.

Congress Dermato-oncology, Spa, Belgium, May 2010.

Basal cell carcinoma, Genetics, Epigenetics and future possible treatments.

Conference New developments in nonmelanoma skin cancer, Integral Cancer Centre Limburg, Sittard, the Netherlands, March 2010. 


\section{Poster presentations}

The effect of topical diclofenac 3\% and calcitriol $3 \mu \mathrm{g} / \mathrm{g}$ on superficial and nodular basal cell carcinoma: a phase II, randomized controlled trial.

$45^{\text {th }}$ annual meeting of the European Society for Dermatological Research, Rotterdam, the Netherlands, September 2015.

A comparison of semi-automated versus pathologist-based manual quantification of immunohistochemical stains in basal cell carcinoma.

Resident scientific meeting (MUMC), Maastricht, the Netherlands, May 2015.

Comprehensive analysis of the basal cell carcinoma DNA methylome using MBD-enriched genomic sequencing.

$14^{\text {th }}$ Annual scientific meeting Dutch Association for Experimental Dermatology, Lunteren, the Netherlands, February 2013.

Comprehensive analysis of the basal cell carcinoma DNA methylome using MBD-enriched genomic sequencing.

Grow Science day, Maastricht, the Netherlands, November 2012.

Topical Diclofenac and Vitamin D as treatment for superficial Basal Cell Carcinoma.

The Dasil $1^{\text {st }}$ annual congress, St. Julians, Malta, November 2012.

Aberrant promoter methylation in Basal Cell Carcinoma.

$13^{\text {th }}$ Annual scientific meeting Dutch Association for Experimental Dermatology, Lunteren, the Netherlands, February 2012.

Aberrant promoter hypermethylation in Basal Cell Carcinoma.

Epigenetic Mechanisms in Health \& Disease. Brussels, Belgium, June 2010.

Aberrant promoter hypermethylation in Basal Cell Carcinoma.

American Association for Cancer Research $101^{\text {st }}$ Annual Meeting, Washington, US, April 2010. 


\section{Dankwoord}

Finis adest rerum. $\mathrm{Na}$ al die jaren is het moment daar om het dankwoord te schrijven. Nu het eenmaal zover is, blijkt dit ook ontzettend moeilijk te zijn. Op de weg die uiteindelijk geleid heeft tot het tot stand komen van dit boekje, zijn er veel mensen die in meer of mindere mate betrokken zijn geweest. Al deze mensen wil ik bedanken voor hun bijdrage, zonder hun zou dit boekje niet zijn geweest wat het nu is geworden. Er zijn een aantal mensen die ik in het bijzonder wil bedanken.

Beste professor van Steensel, beste Maurice. Al sinds mijn semi-arts stage ben jij mijn begeleider, en al vanaf het begin heb je me heel veel vrijheid gegeven om mij te ontwikkelen als onderzoeker. Ik ben je zeer dankbaar voor jouw inspiratie, vertrouwen, antwoord op al mijn vragen en zeker ook de gezellige tijd op het lab. Als grote gemene deler hebben we lekker eten en het praten over eten. Memorabel waren onze noodle soep etentjes in Singapore, natuurlijk gevolgd door een drankje (of twee), waarbij we het onderzoek en de toekomstplannen bespraken. Je uiteindelijke carrière switch naar Dundee en Singapore zorgde er voor dat ik niet meer zomaar je kantoor binnen kon wandelen om een artikel bespreken. Gelukkig mocht ik je via Whatsapp, Facetime, Skype en email blijven stalken. Ik hoop dat we elkaar ook na de afronding van mijn promotie nog regelmatig zullen spreken. Ik zal mezelf in ieder geval regelmatig herinneren aan jouw motto 'Do not focus'.

Beste Dr. Mosterd, lieve Klara. Wat begon als 1 project, zorgde er uit eindelijk voor dat jij mijn co-promoter werd, een super goede zet! Ontzettend bedankt dat jij kritisch en snel al mijn stukken bekeek, waardoor het alleen maar beter en beter werd. Door het overlijden van Kiki werd de afronding van mijn proefschrift voor mij een stuk zwaarder en minder leuk. Zonder jouw hulp en motiverende woorden was die laatste fase nog moeilijker geweest. Jij bent echt een toponderzoeker en voor mij een voorbeeld van hoe je carrière en privé kunt combineren. Dat we nog maar veel mooie projecten samen mogen doen!

Beste Professor Steijlen, allereerst wil ik u bedanken dat $\mathrm{u}$ mij heeft aangenomen voor de opleiding tot dermatoloog en de kans heeft gegeven om binnen de specialisatie ruimte te creëren voor mijn onderzoek. Daarnaast is het ontzettend fijn dat alle ideeën bespreekbaar zijn. Dit heeft er zelfs toe geleid dat ik het MUMC+ Esthetisch Centrum mocht mede-oprichten, hier ben ik u erg dankbaar voor. En ik hoop dat ik u er van heb kunnen overtuigen dat het combineren van de verschillende onderdelen binnen de dermatologie goed mogelijk is.

Beste dr. Nelemans, lieve Patty. Wat ben ik jou dankbaar voor jouw epidemiologische (en ook inhoudelijke) bijdrage aan onze artikelen. Een mailtje sturen met als onderwerp 'HELP' was bij jou voldoende om vervolgens een halve zondag aan de telefoon te hangen om revisies op tijd te kunnen submitten. Onze soms frequente afspraken waren altijd zeer verhelderend, maar vooral ook heel gezellig. Dank voor alles wat ik van je heb geleerd. En Patty, ik beloof je dat ik minder op de P-waarde zal focussen! 
Dat een dermatoloog niet zonder patholoog kan, blijkt mede uit de nauwe samenwerking die er is geweest met de collega's van de pathologie afdeling in het MUMC.

Beste dr. Winnepenninckx, beste Véronique. Wat heb jij een uren samen met mij en later ook Kiki achter de microscoop gezeten! De ene studie was nog niet klaar of we hadden de volgende al klaar liggen. Bedankt voor al jouw tijd en alles wat ik van je heb mogen leren over de dermatopathologie.

Beste professor Zur Hausen. Dank dat u een samenwerking met uw afdeling pathologie heeft mogelijk gemaakt. Heel wat bekende en minder bekende immunohistochemische kleuringen waren er nodig voor de verschillende studies. Uw expertise en die van uw medepathologen bij de beoordeling daarvan zijn van essentieel belang geweest bij het tot stand komen van mijn boekje.

Beste dr. Abdul Hamid, beste Myrurgia, ook jij bedankt voor de samenwerking rondom ons artikel.

Beste professor van Engeland, beste Manon. Jij leerde me kennis maken met de epigenetica en zorgde ervoor dat er een mooie samenwerking ontstond. Dank dat ik ook een beetje bij jullie groep mocht horen.

Alle (oud-) onderzoekers en annalisten van de pathologie, dank voor alle steun \& hulp die ik van jullie heb gehad en natuurlijk ook voor de gezelligheid. Van primers maken tot DNAsequencing, ik bevond mij in een hele nieuwe wereld. Kathleen, dank je dat je altijd tijd voor mij had. En Karin, wat ben ik blij dat ik jou heb leren kennen. Thanx voor je deskundigheid, maar ook voor alle gezellige en soms verdrietige gesprekken. Suzanne, wat was het fijn dat wij een deel konden samenwerken. Dorit, bedankt dat ik je altijd om hulp mocht vragen, jouw promotieboekje gaat helemaal goed komen. Het was een leuke tijd!

Bedankt collega (oud-) AIOS van de pathologie Björn, Lara, Michiel en Jonathan dat jullie mee wilden werken aan mijn studies en heel wat coupes hebben beoordeeld.

Beste Jack Cleutjens, dank voor jouw expertise en ondersteuning bij de semi-automatische beoordelingen van de coupes.

Beste collega's uit Gent, in het bijzonder beste Simon. Kalm en geduldig maakte jij mij wegwijs in de wereld van de bioinformatica. Regelmatig hebben we deadlines vooruitgeschoven, gelukkig is er uiteindelijk mooi werk uit voortgekomen. Ik wens jou een glansrijke carrière in Singapore toe.

Mijn dank is groot voor alle patiënten die wilden participeren in mijn onderzoeken en toestemming hebben gegeven tot publicatie. Zonder hun was een groot deel van dit proefschrift niet mogelijk geweest. 
Ook wil ik alle co-auteurs bedanken voor hun bijdragen aan de artikelen.

Ik dank alle sponsoren voor hun bijdrage in de drukkosten van dit proefschrift.

Beste dr. Kelleners-Smeets, beste Nicole. Bedankt dat je me het gevoel hebt gegeven altijd achter me te staan. Je bent altijd bereid mee te denken. Jouw positiviteit en motiverende woorden zijn heel belangrijk voor me geweest.

Beste drs. Reinders, beste Marieke, dank je voor de fijne samenwerking rondom onze artikelen. Het zijn twee mooie publicaties geworden.

Beste dr. Van Geel, beste Michel. Wat een engelengeduld heb jij! Dank dat je me altijd weer in begrijpelijke taal door de mutatieanalyses heen loodste.

Lieve secretaresses, in het bijzonder Annelies, Petra en Nicole. Bedankt voor alle hulp bij de VitaDi studie, voor het inplannen van soms onmogelijke afspraken met iedereen, maar ook de interesse in mijn onderzoek en een luisterend oor. Bij een gevulde snoeppot zullen jullie me nog vaker zien ;). Jullie zijn toppertjes! Natuurlijk ook een bedankje voor alle baliemedewerksters en verpleegkundigen op de poli dermatologie voor de fijne samenwerking en gezelligheid.

Beste semi-artsen Anne, Ming, Marlou, Annelot en Chantal. Wat was het gezellig om samen met jullie aan de studies te werken. Anne en Ming, waar waren we geweest zonder Whatsapp toen ik met mijn pootje in het gips thuis zat. Secuur werkten jullie aan alle PCR's. Marlou, dank voor jouw inzet rondom de VitaDi studie, de muffins nadat de $100^{\circ}$ patiënt was geïncludeerd hebben zeker tot alertheid bij de artsen geleid! Annelot, dank voor de vele tijd die je stak in de semi-automatische beoordelingen. Chantal, jouw perfectionisme heeft geresulteerd in prachtige figuren. Dank voor al jullie inzet en mooie zorgvuldige werk!

Het derma- lab, daar waar het allemaal ooit begon. Met zijn zessen, of was het nog meer?, in het veel te kleine hok. Reno, Miriam en Renske, dank voor jullie ondersteuning bij de experimenten. Tijs (Tieske) my personal ICT'er! Bedankt dat je altijd weer mijn redder in nood was tijdens een 'computercrisis'. Monique, wat hebben we heerlijk gelachen. Bedankt allemaal voor de fijne tijd.

Beste dr. Van Marion, beste Ariënne. Dank voor de fijne samenwerking! Leuk dat ik in het VieCuri weer van je mocht leren.

Maarten, jou mag ik natuurlijk niet vergeten! Wat ben ik jou dankbaar dat je altijd zo snel het archief in dook om voor mij coupes op te zoeken. Gelukkig kon jij me altijd nog meer bijpraten over alle wedstrijd details van PSV :-). 
Beste Peter Wilms van Kersbergen. Dank dat jij met jouw expertise mijn inleiding en discussie hebt willen controleren en verfijnen op de Engelse taal.

Lieve paranimfen en vriendinnetjes, Marieke en Lieke. Bags, wat is het fijn om naast alle stress rondom het werk alles te kunnen delen en samen te kunnen ontspannen. Alleen de gedachte al aan al onze appjes, gezellige avondjes en weekendjes maakt dat er een glimlach op mijn gezicht verschijnt. Little, wat een lol hebben wij al samen gehad :)! Tranen van geluk, maar soms ook van verdriet hebben gevloeid tijdens onze vele goede gesprekken. Er is niets wat ik niet bij jou kwijt kan. Ik heb genoten van onze tripjes, stapavonden en natuurlijk niet te vergeten de perifere stage in Heerlen. You're the best.

Lieve Karen van Poppelen. Wat ben jij een warm en lief persoon. Fijn om jou als vriendin te hebben. Lieve Marigje, ik ben altijd blij als ik jou om je eerlijke mening kan vragen als deze twijfelaar weer eens niet kan kiezen. Ik heb respect voor hoe hard jij op het lab hebt gewerkt. Jouw proefschrift gaat er ook komen!

Lieve (oud-) mede-promovendi Maud, Janneke, Valerie, Xiaomeng, Aimée, Annet. Dank voor het meedenken tijdens onze meetings en de mogelijkheid om onze onderzoeksfrustraties te delen. We mogen trots zijn op ons onco-clubje! Dat er nog maar heel wat mooie publicaties mogen volgen.

Lieve Kiki, nog steeds vol ongeloof schrijf ik dit stukje voor jou, wetende dat je het nooit zult lezen. Wat een energie-boost gaf het mij toen jij samen met mij aan onze projecten kwam werken. Wat is er nu fijner voor een perfectionist dan te mogen samen werken met iemand die nóg perfectionistischer is. Uren zaten we achter de microscoop, uren werk zaten er in het reviseren van dé VitaDi studie, die uiteindelijk bijna een blok aan ons been werd. Zuur was de champagne die we eigenlijk samen zouden drinken toen het eindelijk geaccepteerd was. Lieve Kiki, ik mis je humor en je lach, je energie en doorzettingsvermogen, je kennis en kritische kijk op het onderzoek. Dank je voor de tijd die we samen hebben doorgebracht. Ik hoop dat het elke dag feest is, daar waar je ook mag zijn.

Lieve collega AIOS dermatologie, dank voor al jullie begrip en steun rondom de afronding van mijn proefschrift. Vanaf nu ben ik er weer elke donderdagmiddag bij! Laten we vooral de trend voortzetten en veel leuke dingen samen blijven doen, ik kijk nu al uit naar het volgende assistentenweekend. Ook dank aan de oud-AIOS, die me al tijdens mijn semi-arts stage enthousiast maakten voor het vak en het onderzoek. De goede gesprekken, gezellige (doordeweekse) stapavonden en assistentenfeestjes waren top!

Lieve Evelien. Wat ben ik trots op je. Niet alleen op de keuzes die je hebt gemaakt, maar vooral ook omdat jij mijn ideeën rondom de vormingeving van mijn proefschrift perfect wist uit te werken tot het boekje zoals het er nu ligt. Het is prachtig geworden. 
Dames van Misspuut Pandora, lieve Pandorianen. Wat was de studententijd een toptijd, maar wat heb ik ook veel van jullie geleerd! De ervaring die ik heb opgedaan tijdens de bestuursjaren en alle commissies komt nog steeds dagelijks van pas. Dank voor alle steun, interesse en natuurlijk de nodige ontspanning op zijn tijd. Vanaf nu is er weer meer tijd! En vergeet niet 'Doe wat je wilt, ...'

Lieve vrienden en vriendinnen, wat ben ik blij dat ondanks de soms spaarzame tijd die we samen kunnen doorbrengen, onze double-dates altijd weer zorgen voor de nodige ontspanning en positieve energie. Karen \& Robert, wanneer gaan we weer mini-hamburgertjes maken? Lydia \& Jurriaan, wat zijn onze avondjes altijd gezellig! Gaan we nog een keer ouderwets Antwerpen op zijn kop zetten met oud \& nieuw? Dirk \& Mariëlle, dank dat jullie altijd zo geïnteresseerd zijn en me ook regelmatig konden voorzien van zakelijke adviezen. Lukas \& Marieke, laten we snel weer een escaperoom plannen!

Lieve Margit, al sinds de eerste onderwijsgroep van geneeskunde is er niemand die mij zo kan laten lachen (op Rolf na dan (:)). Met jou is er altijd iets hilarisch te beleven. Ik heb heel veel respect voor de keuzes die je hebt gemaakt, met jouw carrière gaat het helemaal goed komen. Je mag heel trots zijn op jouw proefschrift.

Lieve Irene. Zo geïnteresseerd en altijd klaar staand voor iedereen. Ik zal nooit vergeten dat jij mij een luisterboek kwam brengen na een van mijn oogoperaties. Je bent een schat!

Lieve Rinske, onze huisvriend! Wat mis ik jou sinds je weg bent uit Maastricht. Al sinds jaar 2 van geneeskunde hebben we lief en leed gedeeld. Regelmatig verlang ik terug naar de tijd dat we de hele zaterdag in de keuken stonden om de meest lekkere gerechten te maken. Gaan we snel weer een gin-tonic drinken? Bedankt dat je er altijd voor me bent.

Lieve schoonfamilie, Ton, Jeanne, Lenneke, Dennis en Carlijn. Regelmatig blonken wij uit door afwezigheid op familieaangelegenheden, als het niet door een naderende deadline was, dan wel door een wedstrijd van PSV. Dank dat jullie altijd begrip en interesse getoond hebben voor mijn onderzoek en het drukke leventje dat Rolf en ik samen leiden.

Lieve familie, ook jullie moet ik bedanken! Wat is het fijn om zo'n gezellige familie te hebben. De laatste jaren had ik vaak te weinig tijd voor jullie, maar vanaf nu kunnen jullie weer op me rekenen.

Lief zusje en lieve Andy. Ondanks dat soms moeilijk uit te leggen was wat het nu precies inhoudt om onderzoek te doen, weet ik dat jullie altijd heel trots zijn op alles wat ik doe. Dank jullie dat Reusje altijd bij jullie terecht kan. 
Lieve pap en mam, het is niet te verwoorden hoe dankbaar ik jullie ben voor alle kansen en steun die jullie mij en ons hebben gegeven. Nooit is iets te veel. Door jullie hebben wij ons volledig op onze carrière kunnen richten. Ik kan me geen lievere en zorgzamere ouders voorstellen. Dit boekje is voor jullie. Ik beloof dat er nu meer rust komt. Love u.

Reusje, onafscheidelijk was je van mijn zijde alle uren die ik thuis achter de laptop doorbracht. Je bent de liefste!

Lieve Rolf, 'Work hard, play hard' lijkt ons nieuwe motto te zijn. De hele weg van student geneeskunde tot de arts die ik nu ben hebben we samen afgelegd. Naast de vele hoge pieken waren er soms ook diepe dalen als mijn gezondheid me weer in de steek liet. Ik heb geen woorden voor hoe belangrijk jouw onvoorwaardelijke steun, onuitputtelijke humor, rust en relativeringsvermogen zijn voor mij. Gelukkig komt er nu meer tijd voor ons.

Amor vincit omnia. 
CV, Grants \& awards, List of publications, Oral \& poster presentations, dankwoord | 231 
